\title{
Mixed Hodge Modules
}

\author{
By \\ Morihiko SAITo*
}

Contents

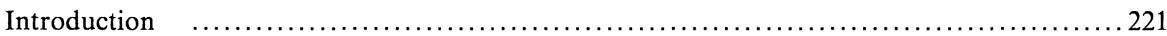

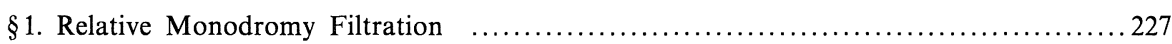

§2. Mixed Hodge Modules on Complex Spaces

(2.a) Vanishing Cycle Functors and Specializations (Divisor Case) $\ldots \ldots \ldots \ldots \ldots \ldots \ldots 236$

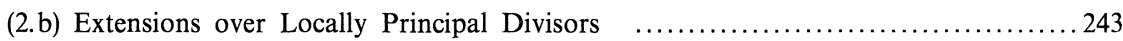

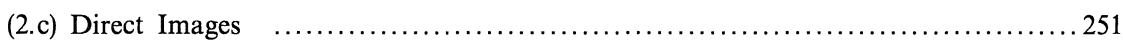



(2.e) Beilinson's Functor and its Applications . .................................261

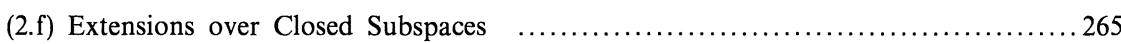

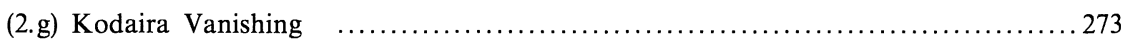

§3. Mixed Hodge Modules of Normal Crossing Type

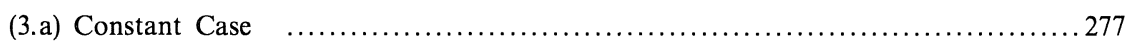

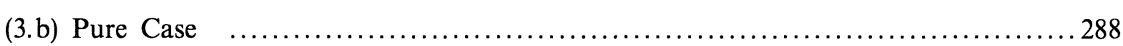

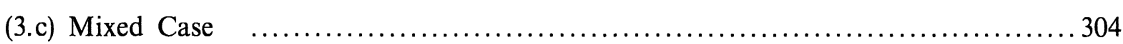

\$4. Algebraic Case ............................................................... 314

\section{Introduction}

In [19] we defined the polarizable Hodge Modules which correspond (philosophically) to the perverse pure complexes in the $l$-adic theory $[3,10]$. For the definition, we used the stability by iterations of the vanishing cycle functors along locally defined holomorphic (or algebraic) functions. Then a polarizable Hodge Module with strict support (i.e. its underlying perverse sheaf is an intersection complex) is generically a polarizable variation of Hodge structure [19, 5.1.10 and 5.2.12]. In this note we show the converse: any polarizable variation of Hodge structure defined on a Zariski open subset can be uniquely (and functorially) extended to a polarizable Hodge Module with strict support. Combined with a result of Kashiwara-Kawai [16] and [19, 5.3.1], we get a natural Hodge structure on $I H^{*}(X, L)$ the intersection cohomology with

Received March 31, 1988.

* Research Institute for Mathematical Sciences, Kyoto University, Kyoto 606, Japan. 
coefficient $L$, if $X$ is compact and bimeromorphic to a compact $\mathbb{K a ̈ h l e r ~ m a n i f o l d ~}$ $\tilde{X}$, i.e. of class $\mathscr{C}$ in the sense of Fujiki (here $\tilde{X}$ can be taken so that we have a projective morphism of $\tilde{X}$ onto $X$ ) and if $L$ is a local system on a Zariski open smooth subset of $X$ and underlies a polarizable variation of Hodge structure. We get also the decomposition theorem of Beilinson-BernsteinDeligne-Gabber for the direct image $f_{*} \mathrm{IC}_{X} L$, if $L$ is as above and $f$ is projective (or proper, in the algebraic case). Here $X$ is assumed irreducible, and $I_{X} L$ is the intersection complex, i.e. $j_{1 *} L[\operatorname{dim} X]$ in the terminology of [3].

In this note we define also the Mixed Hodge Modules which correspond to the perverse mixed complexes $[3,10]$. Roughly speaking, a mixed Hodge Module $\mathscr{M}$ is obtained by extension of polarizable Hodge Modules, i.e. $\mathscr{M}$ is endowed with an increasing filtration $W$, called the weight filtration of $\mathscr{M}$, such that $\mathrm{Gr}_{i}^{W} \mathscr{M}$ are polarizable Hodge Modules of weight $i$. Here the extension can not be arbitrary; we control this using again the vanishing cycle functors. The imposed condition is a natural generalization of that of Steenbrink-Zucker $[22,(3.13)]$, i.e. the existence of the relative monodromy filtration, cf. (2.3.1), and the compatibility condition of the Hodge filtration $F$, the weight filtration $W$ and the filtration $V$ of Malgrange-Kashiwara (indexed by $\mathbb{Q}$ ), cf. (2.2.1) and (2.2.8). We also add the condition for the existence and the uniqueness of the open direct images $j_{*}$ and $j_{\text {! }}$ for open immersions $j$ whose complements are locally principal divisors, and assume that the above conditions are satisfied inductively for iterations of the vanishing cycle functors and the open direct images as above, after taking the smooth pull-backs, cf. (4.2.1). Here we consider the algebraic case for simplicity (in the analytic case we replace $j_{*}$ and $j_{\text {! }}$ by $j_{*} j^{-1}$ and $j_{!} j^{-1}$, cf. (2.17.3).) Let $X$ be a separated and reduced complex algebraic variety, and $\operatorname{MHM}(X)$ the category of mixed Hodge Modules. By definition we have a natural functor rat: $\operatorname{MHM}(X) \rightarrow \operatorname{Perv}\left(\mathbb{Q}_{X}\right)$, where rat means the underlying rational structure and $\operatorname{Perv}\left(\mathbb{Q}_{X}\right)$ is the category of $\mathbb{Q}$-perverse sheaves on $X^{a n}$ with algebraic stratifications [3]. By [19, 5.1.14] and by definition, $\operatorname{MHM}(X)$ is an abelian category, and rat is faithful and exact. The main result of this note is

0.1. Theorem. We have the natural functors $f_{*}, f_{!}, f^{*}, f^{!}, \psi_{g}, \phi_{g, 1}, \mathbb{D}, \bigotimes, \otimes$ and $\mathscr{H}$ om between $D^{b} \mathrm{MHM}(X)$ the derived categories of mixed Hodge Modules, such that these functors are compatible with the corresponding functors on the underlying $\mathbb{Q}$-complexes via:

$$
\text { rat: } D^{b} \operatorname{MHM}(X) \longrightarrow D^{b} \operatorname{Perv}\left(\mathbb{Q}_{X}\right) \stackrel{\text { real }}{\longrightarrow} D_{c}^{b}\left(\mathbb{Q}_{X}\right)
$$

(cf. $[3,3.1 .10]$ for the definition of the functor real), where $f$ is a morphism of algebraic varieties, $g \in \Gamma\left(X, \mathcal{O}_{X}\right)$ and $\phi_{g, 1}=\operatorname{Ker}\left(T_{s}-1\right)$ with $T_{s}$ the semi-simple part of the monodromy $T$ of $\phi_{g}$. 
If $X$ is smooth, $\operatorname{MHM}(X)$ is a full subcategory of $\mathrm{MF}_{r h} \mathrm{~W}\left(\mathscr{D}_{X}, \mathbf{Q}\right)$ $=\{(M, F, K ; W)\}$ the category of filtered regular holonomic $\mathscr{D}_{X}$-Modules with $\mathbb{Q}$-structure given by an isomorphism $\alpha: \mathrm{DR}(M) \cong \mathbf{C} \otimes K$ compatible with finite increasing filtrations $W$ on $M$ and $K \in \operatorname{Perv}\left(\mathbf{Q}_{X}\right)$. Here the morphisms are the pairs of morphisms of filtered $\mathscr{D}_{X}$-Modules and $\mathbf{Q}$-perverse sheaves compatible with $\alpha$ and $W$. By definition $\operatorname{rat}(\mathscr{M})=K$ if $\mathscr{M}=(M, F, K ; W)$. We say that a mixed Hodge Module is smooth (on $X)$ if $\operatorname{rat}(\mathscr{M})[-\operatorname{dim} X]$ is a local system on $X$ and $X$ is smooth. In this case $\mathscr{M}$ is a variation of mixed Hodge structure satisfying some good condition at infinity, i.e. admissible in the sense of Steenbrink-Zucker (one dimensional case) [22] and Kashiwara [13]. For the converse we have (cf. 3.27):

0.2. Theorem. An admissible variation of mixed Hodge structure is a smooth mixed Hodge Module.

As a corollary, a polarizable variation of Hodge structure (and a polarizable Hodge Module) is a mixed Hodge Module. By definition the condition for mixed Hodge Modules is Zariski local. We can construct locally the mixed Hodge Modules by induction on $\operatorname{dim} \operatorname{supp} \mathscr{M}$ by the following:

0.3. Proposition. Let $X$ be an algebraic variety, and $Y$ a principal divisor defined by $g$, i.e. $Y=g^{-1}(0)_{\mathrm{red}}$. Put $U=X \backslash Y$ and let $j: U \rightarrow X$ be the natural inclusion. Then $\operatorname{MHM}(X)$ is equivalent to $\operatorname{MHM}(U, Y)_{e x}$ the category whose objects are $\left(\mathscr{M}^{\prime}, \mathscr{M}^{\prime \prime} ; u, v\right)$ where $\mathscr{M}^{\prime} \in \operatorname{MHM}(U), \mathscr{M}^{\prime \prime} \in \operatorname{MHM}(Y), u \in \operatorname{Hom}\left(\psi_{g, 1} j_{*} \mathscr{M}^{\prime}, \mathscr{M}^{\prime \prime}\right)$ and $v \in \operatorname{Hom}\left(\mathscr{M}^{\prime \prime}, \psi_{g, 1} j_{*} \mathscr{M}^{\prime}(-1)\right)$ such that $v u=N\left(:=\log T_{u} \otimes(2 \pi i)^{-1}\right)$.

Note that the stability of mixed Hodge Modules by $\otimes$ follows from 0.2 and 0.3 , because the admissibility condition is a generic one by [13] and stable by $凶$. To define the functors $f_{*}$ and $f_{!}$in 0.1 , it is enough to define the cohomological ones $\mathscr{H}^{j} f_{*}$ and $\mathscr{H}^{j} f_{\text {! }}$ for quasi-projective morphisms, because we can derive these functors in the affine case and the general case is reduced to this case by [1], cf. 4.3. If $\mathscr{M}$ is pure and $f$ is projective, the cohomological direct images are defined in $[19,5.3 .1]$ and it is not difficult to check the stability of the condition for mixed Hodge Modules, cf. 2.14. Then for $f$ quasiprojective, we define $\mathscr{H}^{i} f_{*}=\left(\mathscr{H}^{i} \bar{f}_{*}\right) j_{*}$, etc. for a factorization $f=\bar{f}^{\circ} j$ such that $\bar{f}$ is projective and $j$ is an open immersion whose complement is a locally principal divisor. Then the independence of the factorization follows from the uniqueness of $j_{*}$ and $j_{!}$. We define $f^{*}$ and $f^{!}$by the adjoint functors of $f_{*}$ and $f_{!}$. Then their existence is reduced to the case where $f$ is a closed immersion $i$ or a projection $p r: X \times Y \rightarrow Y$. For the first case we use an affine covering of the complement $U$ of $X$ and calculate $i^{*}$ and $i^{!}$using the Cech complex, cf. (4.4.1). Here we use the equivalence of categories

$$
i_{*}: D^{b} \operatorname{MHM}(X) \stackrel{\sim}{\longrightarrow} D_{X}^{b} \operatorname{MHM}(Y)
$$


where the right hand side is the full subcategory of $D^{b} \mathrm{MHM}(Y)$ whose objects have cohomological supports in $X$, cf. (4.2.10). In the projection case we show that $p r^{*}$ (resp. $\left.p r^{\prime}\right)$ is represented by the external product $\mathscr{M} \otimes$ for $\mathscr{M} \in D^{b} \operatorname{MHM}(X)$ such that $\operatorname{rat}(\mathscr{M}) \cong \mathbb{Q}_{X}$ (resp. $\left.\mathbb{Q}_{X}\right)$. For the existence of $\mathscr{M}$ we use a kind of Mayer-Vietoris argument, cf. (4.4.2). The stability of $\operatorname{MHM}(X)$ by the vanishing cycle functors is almost clear by definition, and that by the dual functor $\mathbb{D}$ follows from the compatibility of the algebraic and topological dualities with respect to the vanishing cycle functors. For $\otimes$ and $\mathscr{H}$ om we use the well-known formula:

$$
\mathscr{M} \otimes \mathcal{N}=\Delta^{*}(\mathscr{M} \otimes \mathscr{N}), \mathscr{H} \text { om }(\mathscr{M}, \mathcal{N})=\Delta^{!}(\mathbb{D} \mathscr{M} \otimes \mathscr{N}),
$$

where $\Delta: X \rightarrow X \times X$ is the diagonal immersion.

For the proof of 0.2 , we have to prove that an admissible variation of mixed Hodge structure can be extended to a Mixed Hodge Module on $\bar{X}$ a completion of $X$, where we may assume that $\bar{X}$ is smooth and $\bar{X} \backslash X$ is a normal crossing divisor. We first treat the pure case, where a polarizable variation of Hodge structure is naturally extended to a filtered $\mathscr{D} \bar{x}$-Module with $\mathbb{Q}$-structure by the intermediate direct image $j_{! *}=\operatorname{Im}\left(j_{!} \rightarrow j_{*}\right)$, cf. 3.10. We can calculate the vanishing cycle functors along $g$ if the union of $\bar{X} \backslash X$ and $g^{-1}(0)$ is a normal crossing divisor. Here the key point is the compatibility of the $\operatorname{dim} X+1$ filtrations $F, V^{(1)}, \ldots, V^{(\operatorname{dim} X)}$, where $V^{(i)}$ are the filtration $V$ along the coordinate hyperplanes $x_{i}^{-1}(0)$. Then, for the decompositon of $\mathrm{Gr}_{i}^{W} \psi_{g}$, we use a lemma of Kashiwara on nilpotent orbit (cf. 3.19). For a general $g$ we can reduce to the above case using Hironaka's desingularization and the stability by projective direct images. In the mixed case, we use $j_{*}$ for the extension. In this case we prove the compatibility of the $\operatorname{dim} X+2$ filtrations $F, V^{(1)}, \ldots, V^{(\operatorname{dim} X)}$ and $W$, where $W$ on the extension is defined by Kashiwara [13]. Then we can show the stability by the vanishing cycle functors and the open direct images as in the definition of mixed Hodge Modules.

For the proof of 0.3 , we use Beilinson's functor $\xi_{g}$ whose corresponding functor on the $\mathbb{Q}$-complexes is the mapping cone of id $\rightarrow \psi_{g, 1}$ shifted by one to the right. This functor is also used in the proof of the equivalence of categories which appeared in the construction of the functors $i^{*}, i^{!}$.

As a corollary of 0.1 and 0.2 , we get a natural mixed Hodge structure on $H^{*}(X, L)$ if $L$ underlies an admissible variation of mixed Hodge structures. (This result can be generalized to the analytic case, where we assume $X$ has a compactification $\bar{X}$ which is smooth and Kähler, or of class $C$.) Note that this mixed Hodge structure is compatible with the perverse Leray spectral sequences, because, for the composition of $f: X \rightarrow Y$ and $g: Y \rightarrow Z$ and for $\mathscr{M} \in D^{b} \mathrm{MHM}(X)$, the natural truncation $\tau$ on $f_{*} \mathscr{M}$ induces the perverse Leray spectral sequence: 


$$
E_{2}^{p q}=H^{p} g_{*} H^{q} f_{*} \mathscr{M} \Longrightarrow H^{p+q}(g f)_{*} \mathscr{M} \quad \text { in } \operatorname{MHM}(Z)
$$

We say that $\mathscr{M} \in D^{b} \mathrm{MHM}(X)$ is of weight $\leq n$ (resp. $\geq n$ ) if $\mathrm{Gr}_{i}^{W} H^{j} \mathscr{M}=0$ for $i>j+n$ (resp. $i<j+n$ ). This condition is stable by $f_{!}, f^{*}\left(\right.$ resp. $\left.f_{*}, f^{!}\right)$, cf. 2.26. We say that $\mathscr{M} \in D^{b} \operatorname{MHM}(X)$ is pure of weight $n$ if $\operatorname{Gr}_{i}^{W} H^{j} \mathscr{M}=0$ for $i \neq j+n$. Then this condition is stable by the direct images for proper morphisms. Moreover a pure complex $\mathscr{M}$ is isomorphic to the direct sum of $H^{j} \mathscr{M}[-j]$, because $\operatorname{Ext}^{j}(\mathscr{M}, \mathscr{N})=0$ for $\mathscr{M}, \mathscr{N}$ pure of weight $m, n$ with $m<n$ $+j$, cf. 4.25. These facts are analogue of the results in [3].

For an algebraic variety $X$, we define $\mathbb{Q}_{X}^{H}=a_{X}^{*} \mathbb{Q}_{p t}^{H}$, where $a_{X}: X \rightarrow p t$ $\left(=\operatorname{Spec} \mathbb{C}\right.$ ) and $\mathbb{Q}_{p t}^{H}=(\mathbb{C}, F, \mathbb{Q}, W)$ with $\mathrm{Gr}_{i}^{F}=\mathrm{Gr}_{i}^{W}=0$ for $i \neq 0$. Then for an irreducible closed subvariety $Z$ of dimension $d$ in $X$, we can define the Hodge cycle class $\mathrm{cl}_{Z}^{H}$ of $Z$ in

(*) $\operatorname{Hom}\left(\mathbb{Q}_{X}^{H},\left(\mathbb{D} \mathbb{Q}_{X}^{H}\right)(-d)[-2 d]\right)=\operatorname{Hom}\left(\mathbb{Q}_{p t}^{H},\left(a_{X}\right)_{*}\left(\mathbb{D} \mathbf{Q}_{X}^{H}\right)(-d)[-2 d]\right)$

using a natural morphism $\mathbb{Q}_{Z}^{H}[d] \rightarrow \operatorname{IC}_{Z} \mathbb{Q}^{H}\left(:=j_{1 *} \mathbb{Q}_{Z_{\text {reg }}}[d]\right)$ and its dual morphism, where $j: Z_{\text {reg }} \rightarrow Z$. If $X$ is smooth, $\mathbb{D} \mathbf{Q}_{X}^{H}=\mathbb{Q}_{X}^{H}\left(d_{X}\right)\left[2 d_{X}\right]$ with $d_{X}$ $=\operatorname{dim} X$, and the right hand side of $(*)$ is isomorphic to the $\mathbb{Q}$-Deligne cohomology if $X$ is smooth proper.

The plan of this note is as follows.

In $\S 1$, we develop the theory of relative monodromy filtration in the exact categories, which gives easily the compatibility of some results with the Hodge filtration. The main results are Kashiwara's canonical splitting [13, 3.2.9] where we use the primitive decomposition for the proof (cf. 1.5), and the generalization of Steenbrink-Zucker's formula for the weight filtration on the open direct images $[22,(4.8)]$ to the case where neither can nor Var are bijective (cf. 1.9).

In $\S 2$, we study the mixed Hodge Modules in the analytic case. Almost all the arguments in this section can be applied also to the algebraic case, and the results which are particular only to the algebraic case will be written in $\S 4$. (Note that the definition of mixed Hodge Module is different in the algebraic and analytic case due to the difference of the topologies: in the algebraic case the mixed Hodge Modules are always assumed to be extendable and polarizable.) In (2.a) we introduce the notion: the vanishing cycle functors are well-defined along a holomorphic function, which is a natural generalization of $[22,(3.13)]$, and we prove the stability by dual and subquotient in $\mathrm{MHW}(X)$ the category whose objects are obtained by extensions of Hodge Modules, i.e. $\mathscr{M} \in \mathrm{MHW}(X)$ has the weight filtration $W$ such that $\operatorname{Gr}_{i}^{W} \mathscr{M} \in \mathrm{MH}(X, i)$. In (2.b), we study the extensions over the locally principal divisors in the case of $\operatorname{MHW}(X)$, and prove an analogue of Deligne-MacPherson-Verdier's formula [23] where the vanishing cycle functors are used for the gluing, cf. 2.8. Here we use 1.9 to define $W$ on 
the extension. We also show the polarizability of the open direct images (cf. 2.11) where we use Verdier's specialization. In (2.c) we show the stability of the well-definedness of vanishing cycle functors by the proper direct images, assuming some stability of Hodge Modules which appear as the graduation by $W$, cf. 2.14. In (2.d) we define the mixed Hodge Modules in the analytic case, and construct the cohomological direct images and pull-backs for some morphisms. In (2.e) we define Beilinson's functor $\xi_{g}$ in the category of mixed Hodge Modules. Using this, we prove some equivalence of categories associated to a closed immersion (cf. (2.23.2)) and the estimation of the weight for the cohomological direct images and pull-backs, cf. 2.26. In (2.f) we show the analogue of Deligne-MacPherson-Verdier's formula in the case of mixed Hodge Modules, cf. 2.28. We also prove MacPherson's version, cf. 2.32. In (2.g) we prove the generalization of the Kodaira vanishing (cf. 2.33) which implies the Ohsawa-Kollár vanishing, cf. 2.34, and the Guillen-Navarro-Puerta vanishing, cf. (2.33.2).

The aim of $\S 3$ is to relate the variations of (mixed) Hodge structures to the (mixed) Hodge Modules. In (3.a) we calculate the vanishing cycle functors for the perverse sheaves in the normal crossing case, cf. 3.3-5. As a corollary, we prove that the (polarizable) mixed Hodge Modules on a point are identified with the (polarizable) Q-mixed Hodge structures, cf. 3.9. In (3.b) we study the intermediate direct image of a polarizable variation of Hodge structure. We show the compatibility of $F, V^{(i)}(1 \leq i \leq \operatorname{dim} X)$, cf. 3.12 , and calculate the vanishing cycle functors, cf, 3.17. Then, combining with Kashiwara's result on nilpotent orbit, we prove the stability by vanishing cycle functors and this intermediate direct image is a polarizable Hodge Module. In (3.c) we study the usual direct image of an admissible variation of mixed Hodge structures. Here we also show the compatibility of $F, W, V^{(i)}(1 \leq i \leq \operatorname{dim} X)$, and calculate the vanishing cycle functors. In this case the difficulty is to show the existence of the relative monodromy filtration and (3.23.14) is proved for this purpose.

In $\S 4$ we study the mixed Hodge Modules on algebraic varieties. We give the second definition of mixed Hodge Modules to show the equivalence of categories (4.2.4), and prove the equivalence of these two definitions, cf. 4.2. We define the direct images in 4.3 using a result of Beilinson $[1, \S 3]$, and the pull-backs in 4.3 as the adjoint functors of the direct images. The Hodge cycle class of an irreducible closed subvariety is defined in 4.5.

I would like to thank Professors P. Deligne and M. Kashiwara for useful discussions and their stimulations. A part of this work was done during my stay at the Institute for Advanced Study in 1985/86. I would like to thank the staff of the institute for the hospitality. 


\section{§1. Relative Monodromy Filtration (cf. [10, 13, 22, etc.])}

1.1. Let $\mathscr{C}$ be an exact category, i.e. there is an abelian category $\mathscr{A}$ such that $\mathscr{C}$ is an additive full subcategory of $\mathscr{A}$ stable by extensions, and a short sequence in $\mathscr{C}$ is called exact if it is exact in $\mathscr{A}$. We assume $\mathscr{C}$ has an additive automorphism $S$ which is extended to $\mathscr{A}$. (For example, $\mathscr{C}$ is the category of filtered objects of an abelian category and $S$ is a shift of filtration; or $S$ $=\mathrm{id}$ ). Let $L$ be a finite increasing filtration of $M \in \mathscr{C}$ (cf. $[19,1.3 .1]$ ), and $N:(M, L) \rightarrow S^{-1}(M, L)$ a filtered morphism such that $N^{i}=0$ for $i \gg 0$, where the filtration $L=S^{j} L$ on $S^{j} M$ is defined by $L_{i}\left(S^{j} M\right)=S^{j}\left(L_{i} M\right)$ for $i, j \in \mathbb{Z}$. Then there exists at most one finite increasing filtration $W$ of $(M, L)$ [loc. cit], called the relative monodromy filtration, such that:

(1.1.1) $\quad N$ induces a morphism $N:(M ; L, W) \longrightarrow S^{-1}(M ; L, W[2])$,

$$
N^{i}: \mathrm{Gr}_{i+k}^{W} \mathrm{Gr}_{k}^{L} M \stackrel{\sim}{\longrightarrow} S^{-i} \mathrm{Gr}_{-i+k}^{W} \mathrm{Gr}_{k}^{L} M \quad \text { for } i>0,
$$

where $W[m]_{i}=W_{i-m}$. In fact, in the case $\mathscr{C}=\mathscr{A}$, we have Deligne's inductive formula $[10,1.6 .13]$ (if $W$ exists):

$$
\begin{array}{cc}
W_{-i+k} L_{k} M=W_{-i+k} L_{k-1} M+N^{i}\left(S^{i} W_{i+k} L_{k} M\right) & (i>0), \\
W_{i+k} L_{k} M=\operatorname{Ker}\left(N^{i+1}: L_{k} M \longrightarrow S^{-i-1}\left(L_{k} M / W_{-i-2+k} L_{k} M\right)\right) & (i \geq 0),
\end{array}
$$

where $W_{i+k} L_{k} M=L_{k} M(i \gg 0)$ follows from (1.1.4). In general, the relative monodromy filtration $W$ of $(M, L)$ in $\mathscr{C}$ is the relative monodromy filtration of $(M, L)$ in $\mathscr{A}$ such that $\mathrm{Gr}_{i}^{W} \mathrm{Gr}_{k}^{L} M \in \mathscr{C}$ (cf. [19, 1.3.2]). In the case $\mathrm{Gr}_{k}^{L} M=0$ for $k \neq 0$ (or $k \neq n$, more generally), $W$ is called the monodromy filtration (shifted by $n$, if $n \neq 0$ ) of $M$, and we define the primitive part by

$$
P \mathrm{Gr}_{i}^{W} M=\operatorname{Ker}\left(\mathrm{Gr}^{W} N^{i+1}: \mathrm{Gr}_{i}^{W} M \longrightarrow S^{-i-1} \mathrm{Gr}_{-i-2}^{W} M\right)
$$

for $i \geq 0$ and 0 otherwise,

if $\mathrm{Gr}^{W} N^{i+1}$ has the kernel in $\mathscr{C}$. In this case $\operatorname{PGr}_{i}^{W} M$ is the kernel in $\mathscr{A}$ (and belongs to $\mathscr{C}$ ), because $\mathrm{Gr}^{W} N^{i+2}: \mathrm{Gr}_{i+2}^{W} M \sim S^{-i-2} \mathrm{Gr}_{-i-2}^{W} M$ (hence $\mathrm{Gr}^{W} N^{i+1}$ is a strict epimorphism by definition). In particular, we have the Lefschetz decomposition:

$$
\sum \mathrm{Gr}^{W} N^{m}: \oplus S^{m} P \mathrm{Gr}_{i+2 m}^{W} M \stackrel{\sim}{\longrightarrow} \mathrm{Gr}_{i}^{W} M
$$

where the summation is taken over $m \geq 0$ such that $i+m \geq 0$.

In general the relative monodromy filtration does not always exist. In the case of abelian category, an inductive criterion is given in $[22,(2.20)]$ :

1.2. Lemma. With the notation as above, assume $\mathscr{C}=\mathscr{A}$ and the relative 
monodromy filtration of $\left(L_{k-1} M, L\right)$ exists, then it exists on $\left(L_{k}, M\right)$ iff:

(1.2.1) $N^{i+1}: \operatorname{Ker}\left(N^{i+1}: S^{i+1} \mathrm{Gr}_{k}^{L} M \longrightarrow \mathrm{Gr}_{k}^{L} M\right) \longrightarrow L_{k-1} /\left(N^{i+1}\left(S^{i+1} L_{k-1} M\right)\right.$

$\left.+W_{-i-2+k} L_{k-1} M\right)$ is zero for $i \geq 0$.

(See [loc. cit] for a proof.)

1.3. Remark. It is easy to see that (1.2.1) is a necessary condition, because

$$
\operatorname{Ker}\left(N^{i+1}: \mathrm{Gr}_{k}^{L} M \longrightarrow S^{-i-1} \mathrm{Gr}_{k}^{L} M\right) \subset W_{i+k} \mathrm{Gr}_{k}^{L} M .
$$

(In fact, $N^{i+1}: \mathrm{Gr}_{i+1+l+k}^{W} \mathrm{Gr}_{k}^{L} M \rightarrow S^{-i-1} \mathrm{Gr}_{-i-1+l+k}^{W} \mathrm{Gr}_{k}^{L} M(l \geq 0)$ and $N^{i+1}$ : $\mathrm{Gr}_{k}^{L} M / W_{i+k} \mathrm{Gr}_{k}^{L} \rightarrow S^{-i-1}\left(\mathrm{Gr}_{k}^{L} M / W_{-i-2+k} \mathrm{Gr}_{k}^{L} M\right)$ are injective.) For the converse, we have a direct proof, if $\mathscr{C}=\mathscr{A}$ is the abelian category of $R$-modules for $R$ an algebra over a field $K$ and $S=$ id. In fact, the assertion is reduced to the case $R=K$ by (1.1.3-4), then we may assume $\mathscr{C}$ is a semi-simple abelian category (i.e. every exact sequence splits). We shall construct a splitting $s: \bar{M}:=\mathrm{Gr}_{k}^{L} M$ $\rightarrow L_{k} M$ such that $(s N-N s)\left(W_{i} \bar{M}\right) \subset W_{i-2} L_{k-1} M$, where $W$ is the monodromy filtration of $\bar{M}$ shifted by $k$. First we take $\bar{s}_{i}: \operatorname{PGr}_{i+k}^{W} \bar{M} \rightarrow W_{i+k} \bar{M}$, such that its composition with the projection $W_{i+k} \bar{M} \rightarrow \mathrm{Gr}_{i+k}^{W} \bar{M}$ is the natural inclusion. We can modify $\bar{s}_{i}$ inductively so that $\operatorname{Im}\left(N^{i+1} \bar{s}_{i}\right) \subset W_{-l-i-2+k} \bar{M}$ for any $l>0$, i.e. $\operatorname{Im}\left(\bar{s}_{i}\right) \subset \operatorname{Ker} N^{i+1}$. By (1.2.1) we can lift $\bar{s}_{i}$ to a morphism $s_{i}: P \operatorname{Gr}_{i+k}^{W} \bar{M} \rightarrow$ $W_{i+k} L_{k} M$ such that $\operatorname{Im}\left(N^{i+1} s_{i}\right) \subset W_{-i-2+k} L_{k-1} M$. By the Lefschetz decomposition, we have an isomorphism:

$$
\sum_{0 \leq m \leq i} N^{m} \bar{s}_{i}: \underset{0 \leq m \leq i}{\oplus} P \operatorname{Gr}_{i+k}^{W} \bar{M} \longrightarrow \bar{M} .
$$

We get the section $s$ by replacing $\bar{s}_{i}$ with $s_{i}$ in the above morphism. Then $(N s$ $-s N)\left(W_{i} \bar{M}\right) \subset W_{i-2} L_{k-1} M$ is clear and we get the relative monodromy filtration of $\left(L_{k} M, L\right)$ by

$$
W_{i} L_{k} M=W_{i} L_{k-1} M+s\left(W_{i} \bar{M}\right) .
$$

(This argument can be generalized to the filtered case.)

1.4. Remark. With the notation of 1.1 , the relative monodromy filtration of $(M, L)$ exists, iff $(1.2 .1)$ is satisfied in $\mathscr{A}$ and each $\mathrm{Gr}_{k}^{L} M$ has the monodromy filtration in $\mathscr{C}$ (i.e. $\mathrm{Gr}_{i}^{W} \mathrm{Gr}_{k}^{L} M \in \mathscr{C}$ ).

The following proposition and two corollaries are variants of Kashiwara's result [13].

1.5. Propositon (cf. [13, Th. 3.2.9]). With the notation of 1.1 , assume the relative monodromy filtration $W$ of $(M, L)$ exists and

$$
P \mathrm{Gr}_{i+k}^{W} \mathrm{Gr}_{k}^{L} M:=\operatorname{Ker}\left(\mathrm{Gr}^{W} \mathrm{Gr}^{L} N^{i+1}: \mathrm{Gr}_{i+k}^{W} \mathrm{Gr}_{k}^{L} M \longrightarrow S^{-i-1} \mathrm{Gr}_{-i-2+k}^{W} \mathrm{Gr}_{k}^{L} M\right)
$$


belongs to $\mathscr{C}$ (i.e. the kernel exists in $\mathscr{C}, c f$. (1.1.5)). Then there exists a unique splitting of $\left(\mathrm{Gr}_{i}^{W} M, L\right)$ for any $i$, satisfying the following conditions:

Let $s_{i, k}: \mathrm{Gr}_{i}^{W} \mathrm{Gr}_{k}^{L} M \rightarrow \mathrm{Gr}_{i}^{W} L_{k} M$ be the section corresponding to the splitting, and $s_{i+k, k}^{\prime}: \mathrm{PGr}_{i+k}^{W} \mathrm{Gr}_{k}^{L} M \rightarrow \mathrm{Gr}_{i+k}^{W} L_{k} M$ the restriction of $s_{i+k, k}$ to the primitive part. Then:

(1.5.1) $\operatorname{Im}\left(s_{i+k, k}\right)=\sum \operatorname{Im}\left(N^{m} s_{j+k, k}^{\prime}\right) \quad$ (the summation is taken over $j, m \geq 0$ such that $m \leq j, j-2 m=i)$,

(1.5.2) $\operatorname{Im}\left(s_{i+k, k}^{\prime}\right)=\operatorname{Ker}\left(N^{i+1}: \mathrm{Gr}_{i+k}^{W} L_{k} M \longrightarrow S^{-i-1}\left(\mathrm{Gr}_{-i-2+k}^{W} L_{k} M / V_{i+k, k}\right)\right)$ where $V_{i+k, k}=\sum \operatorname{Im}\left(N^{m} s_{j+l, l}^{\prime}\right) \quad$ (the summation is taken over $j, m \geq 0$, $l \leq k-2$ such that $m \leq j, j+l<i+k, j+l-2 m=k-i-2)$.

Moreover, $N^{m} s_{j+k, k}^{\prime}: P \mathrm{Gr}_{j+k}^{W} \mathrm{Gr}_{k}^{L} M \rightarrow S^{-m} \mathrm{Gr}_{j+k-2 m}^{W} L_{k} M$ are strict monomorphisms for $m \leq j$, the summations in (1.5.1-2) are direct sums and the morphism in the right hand side of (1.5.2) is a strict epimorphism.

Proof. If there is a splitting of $\left(\mathrm{Gr}_{i}^{W} M, L\right)$ satisfying (1.5.1) in $\mathscr{A}$, the Lefschetz decomposition (cf. (1.1.6)) implies the isomorphism:

$$
\sum N^{m} s_{j+l, l}^{\prime}: \oplus S^{m} P \mathrm{Gr}_{j+l}^{W} \mathrm{Gr}_{l}^{L} M \longrightarrow \mathrm{Gr}_{i}^{W} M
$$

where the summation is taken over $j, m \geq 0, l \in \mathbb{Z}$ such that $m \leq j, j+l-2 m$ $=i$. In particular $N^{m} s_{j+l, l}^{\prime}$ are strict monomorphisms for $m \leq j$ and the summations in (1.5.1-2) are direct sums. Therefore it is enough to show that the morphism in (1.5.2) is surjective in $\mathscr{A}$ and the right hand side of (1.5.2) projects isomorphically onto $\mathrm{PGr}_{j+k}^{W} \mathrm{Gr}_{k}^{L} M$ by induction on $k$, because the sections are uniquely determined by their image and $V_{i+k, k}$ is defined by $s_{j+l, l}^{\prime}$ with $l<k$.

Assume the assertion is verified for $(j, l)$ such that $l<k$ or $j>i, l=k$. We define a morphism by

$$
\tilde{s}_{i+k, k}:=\oplus N^{m} s_{j+l, l}^{\prime}: U_{i+k, k}:=\oplus S^{m} P \mathrm{Gr}_{j+l}^{W} \mathrm{Gr}_{l}^{L} M \longrightarrow \mathrm{Gr}_{i+k}^{W} L_{k} M
$$

where the summation is taken over $j, m \geq 0, l \leq k$ such that $m \leq j, j+l-2 m$ $=i+k, j>i($ if $l=k$ ). Then the Lefschetz decomposition implies the short exact sequence:

$$
0 \longrightarrow U_{i+k, k} \stackrel{\tilde{s}_{\imath+k, k}}{\longrightarrow} \mathrm{Gr}_{i+k}^{W} L_{k} M \stackrel{\mathrm{pr}}{\longrightarrow} P \mathrm{Gr}_{i+k}^{W} \mathrm{Gr}_{k}^{L} M \longrightarrow 0
$$

where $\mathrm{pr}$ is the composition: $\mathrm{Gr}_{i+k}^{W} L_{k} M \rightarrow \mathrm{Gr}_{i+k}^{W} \mathrm{Gr}_{k}^{L} M \rightarrow P \mathrm{Gr}_{i+k}^{W} \mathrm{Gr}_{k}^{L} M$. Moreover $N^{i+1} \tilde{s}_{i+k, k}: U_{i+k, k} \rightarrow S^{-i-1} \mathrm{Gr}_{-i-2+k}^{W} L_{k} M$ is injective (in $\mathscr{A}$ ) and $V_{i+k, k}$ is a complement to its image. Then the assertion follows from the diagram in $\mathscr{A}$ : 


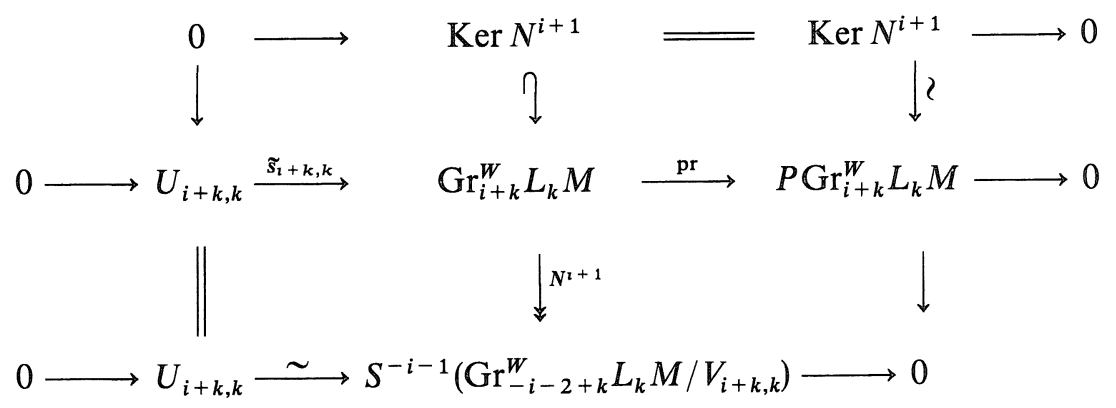

1.6. Corollary. Let $(M ; L, W, N)$ and $\left(M^{\prime} ; L, W, N\right)$ be as in 1.1 , and $u:(M ; L, N) \rightarrow\left(M^{\prime} ; L, N\right)$ a morphism compatible with $L, N$. If $\mathrm{Gr}_{k}^{L} u$ induces (1.6.1) $\quad \mathrm{Gr}_{k}^{L} u:\left(\mathrm{Gr}_{k}^{L} M, W\right) \longrightarrow\left(\mathrm{Gr}_{k}^{L} M^{\prime}, W[1]\right) \quad$ for any $k$, $u \quad$ induces $u:(M ; L, W) \rightarrow\left(M^{\prime} ; L, W[1]\right)$. (Note that $u$ induces always $u:(M ; L, W) \rightarrow\left(M^{\prime} ; L, W\right)$ by $(1.1 .3-4)$.)

Proof. We may assume $\mathscr{C}=\mathscr{A}$, cf. 1.1. We prove by induction on $k$ :

$$
\mathrm{Gr}^{W} u: \mathrm{Gr}_{i}^{W} L_{k} M \longrightarrow \mathrm{Gr}_{i}^{W} L_{k} M^{\prime} \text { are zero for any } i \text {. }
$$

Let $s_{i+k, k}^{\prime}$ and $t_{i+k, k}^{\prime}$ be the sections for $M$ and $M^{\prime}$ corresponding to the splitting in 1.5. Then it is enough to show the composition:

$$
P \mathrm{Gr}_{i+k}^{W} \mathrm{Gr}_{k}^{L} K \stackrel{s^{\prime}}{\longrightarrow} \mathrm{Gr}_{i+k}^{W} L_{k} M \stackrel{u}{\longrightarrow} \mathrm{Gr}_{i+k}^{W} L_{k} M^{\prime}
$$

is zero. Because $\operatorname{Im}\left(N^{i+1} s_{i+k, k}^{\prime}\right) \subset \mathrm{Gr}_{-i-2+k}^{W} L_{k-2} M$, the composition:

$$
\begin{aligned}
& N^{i+1} \circ \mathrm{Gr}^{W} u \circ s_{i+k, k}^{\prime}=\mathrm{Gr}^{W} u \circ N^{i+1} \circ s_{i+k, k}^{\prime} \\
& S^{i+1} P \mathrm{Gr}_{i+k}^{W} \mathrm{Gr}_{k}^{L} M \longrightarrow \mathrm{Gr}_{-i-2+k}^{W} L_{k} M^{\prime}
\end{aligned}
$$

is zero by inductive hypothesis. This implies

$$
\operatorname{Im}\left(\mathrm{Gr}^{W} u \circ s_{i+k, k}^{\prime}\right) \subset \operatorname{Im}\left(t_{i+k, k}^{\prime}\right) .
$$

Therefore it is enough to show

$$
\mathrm{Gr}^{W} \mathrm{Gr}^{L} u: P \mathrm{Gr}_{i+k}^{W} \mathrm{Gr}_{k}^{L} M \longrightarrow \mathrm{Gr}_{i+k}^{W} \mathrm{Gr}_{k}^{L} M^{\prime}
$$

is zero, because $\operatorname{Im}\left(t_{i+k, k}^{\prime}\right)$ projects isomorphically onto $P \mathrm{Gr}_{i+k}^{W} \mathrm{Gr}_{k}^{L} M^{\prime}$. But this follows from the assumption (1.6.1).

1.7. Corollary (cf. $\left[13\right.$, Lemma 3.3.2]). Let $(M ; L, W, N)$ and $\left(M^{\prime} ; L, W, N\right)$ be as above, and $u:(M, L) \rightarrow\left(M^{\prime}, L\right)$ and $v:\left(M^{\prime}, L\right) \rightarrow S^{-1}(M, L)$ filtered morphisms such that $u v=N$ and $v u=N$. Assume:

$$
\mathrm{Gr}_{K}^{L} M^{\prime}=\operatorname{Im}\left(\mathrm{Gr}_{k}^{L} u\right) \oplus \operatorname{Ker}\left(\mathrm{Gr}_{k}^{L} v\right) \quad \text { in } \mathscr{A} \text { (cf. 1.1) }
$$


for any $k$. Then $u$ and $v$ induce the morphisms:

$$
u:(M ; L, W) \longrightarrow\left(M^{\prime} ; L, W[1]\right), \quad v:\left(M^{\prime} ; L, W\right) \longrightarrow S^{-1}(M ; L, W[1]) .
$$

Proof. We may assume $\mathscr{C}=\mathscr{A}$. Then the assertion follows from the next proposition :

1.8. Proposition. Let $u: M \rightarrow M^{\prime}$ and $v: M^{\prime} \rightarrow S^{-1} M$ be morphisms in $\mathscr{A}$ (cf. 1.1) such that $N=v u$ and $N=u v$ are nilpotent. Let $W$ be the monodromy filtration of $M$ and $M^{\prime}$. Then the condition:

$$
M^{\prime}=\operatorname{Im} u \oplus \operatorname{Ker} v
$$

implies:

(1.8.2) $u$ and $v$ induce strict morphisms

$u:(M, W) \longrightarrow\left(M^{\prime}, W[1]\right), \quad v:\left(M^{\prime}, W\right) \longrightarrow S^{-1}(M, W[1])$,

(1.8.3) $\mathrm{Gr}_{i+1}^{W} u: \mathrm{Gr}_{i+1}^{W} M \longrightarrow \mathrm{Gr}_{i}^{W} M^{\prime}$ is surjective for $i>0$,

$\mathrm{Gr}_{i}^{W} v: \mathrm{Gr}_{i}^{W} M^{\prime} \longrightarrow S^{-1} \mathrm{Gr}_{i-1}^{W} M$ is injective for $i<0$.

(1.8.4) $\quad u: M / W_{1} M \longrightarrow M^{\prime} / W_{0} M^{\prime}$ is surjective

$v: W_{-1} M^{\prime} \longrightarrow S^{-1} W_{-2} M$ is injective.

Conversely, let $u:(M, W) \rightarrow\left(M^{\prime}, W[1]\right)$ and $v:\left(M^{\prime}, W\right) \rightarrow S^{-1}(M, W[1])$ be filtered morphisms in $\mathscr{A}$, where $W$ is a finite increasing filtration on $M$ and $M^{\prime}$ (in particular, $N:=v u$ and $N:=u v$ are nilpotent). Assume (1.8.4) holds and $W$ on $M$ is the monodromy filtration, then so is $W$ on $M^{\prime}$ and (1.8.1-3) hold.

Proof. If $(1.8 .1)$ holds, $\left(M \rightleftarrows M^{\prime}\right)$ is the direct sum of $(M \rightleftarrows \operatorname{Im} u)$ and $(0 \rightleftarrows \operatorname{Ker} v)$, and the action of $N$ and the filtration $W$ are compatible with this decomposition. Then (1.8.2) follows from [19, Lemma 5.1.12], and (1.8.3) (hence (1.8.4)) from

$$
\begin{aligned}
\operatorname{Coker}\left(\operatorname{Gr}_{i+1}^{W} u\right) & \simeq \operatorname{Gr}_{i}^{W} \operatorname{Coker} u \simeq \operatorname{Gr}_{i}^{W} \operatorname{Ker} v \\
& \simeq \operatorname{Ker}\left(\operatorname{Gr}_{i}^{W} v\right)=0 \text { for } i \neq 0 .
\end{aligned}
$$

Conversely, the isomorphisms $\mathrm{Gr}^{W} N^{i}: \mathrm{Gr}_{i}^{W} M \Im S^{-i} \mathrm{Gr}_{-i}^{W} M$ imply:

$u:\left(M / W_{0} M, W\right) \longrightarrow\left(M^{\prime} / W_{-1} M^{\prime}, W[1]\right)$ is strictly injective,

$v:\left(W_{0} M^{\prime}, W\right) \longrightarrow S^{-1}\left(W_{-1} M, W[1]\right)$ is strictly surjective,

because $\operatorname{Gr}_{i+1}^{W} u$ is injective for $j \geq 0$ and $\mathrm{Gr}_{i}^{W} v$ is surjective for $i \leq 0$. Therefore

$$
\begin{aligned}
& u:\left(M / W_{1} M, W\right) \stackrel{\sim}{\longrightarrow}\left(M^{\prime} / W_{0} M^{\prime}, W[1]\right) \\
& v:\left(W_{-1} M^{\prime}, W\right) \stackrel{\sim}{\longrightarrow} S^{-1}\left(W_{-2} M, W[1]\right)
\end{aligned}
$$


by the condition (1.8.4), and we get:

(1.8.5) $\mathrm{Gr}_{i+1}^{W} u$ is bijective for $i>0$, injective for $i=0$ and surjective for $i<0$.

(1.8.6) $\operatorname{Gr}_{i}^{W} v$ is injective for $i>0$, surjective for $i=0$ and bijective for $i<0$.

In particular, $u$ and $v$ are strict, because (1.8.5) implies:

$$
\begin{aligned}
& u: M / W_{i+1} M \longrightarrow M^{\prime} / W_{i} M^{\prime} \text { is injective for } i \geq 0, \\
& u: W_{i+1} M \longrightarrow W_{i} M^{\prime} \text { is surjective for } i<0
\end{aligned}
$$

(same for $v$ ). Moreover we have

$$
\operatorname{Coker}\left(\mathrm{Gr}_{i+1}^{W} u\right)=\operatorname{Ker}\left(\mathrm{Gr}_{i}^{W} v\right)=0 \text { for } i \neq 0
$$

by (1.8.5-6), and the isomorphism

$$
\mathrm{Gr}_{1}^{W} N=\mathrm{Gr}_{0}^{W} v \circ \mathrm{Gr}_{1}^{W} u: \mathrm{Gr}_{1}^{W} M \longrightarrow S^{-1} \mathrm{Gr}_{-1}^{W} M
$$

implies the decomposition:

$$
\mathrm{Gr}^{W} M^{\prime}=\operatorname{Im}\left(\mathrm{Gr}^{W} u\right) \oplus \operatorname{Ker}\left(\mathrm{Gr}^{W} v\right) .
$$

Then (1.8.1) follows from (1.8.2) and (1.8.7), and $W$ on $M^{\prime}$ is the monodromy filtration, because the action of $\mathrm{Gr}^{W} N$ is compatible with the decomposition (1.8.7) and

$$
\operatorname{Coim}\left(\mathrm{Gr}^{W} u\right) \simeq \operatorname{Coim}\left(\mathrm{Gr}^{W} N: \mathrm{Gr}^{W} M \longrightarrow S^{-1} \mathrm{Gr}^{W[2]} M\right) .
$$

The following is a generalization of $[22,(4.11)]$.

1.9. Corollary. Let $u:(M, L) \rightarrow\left(M^{\prime}, L\right)$ and $v:\left(M^{\prime}, L\right) \rightarrow S^{-1}(M, L)$ be filtered morphisms such that $N=v u$ and $N=v u$ are nilpotent on $M$ and $M^{\prime}$, where $L$ is a finite increasing filtration and $M, M^{\prime}$ are objects of $\mathscr{A}(c f .1 .1)$. Let $W$ be a finite increasing filtration of $M, M^{\prime}$ such that $u\left(W_{i} M\right) \subset W_{i-1} M^{\prime}, \quad v\left(W_{i} M^{\prime}\right)$ $\subset S^{-1} W_{i-1} M$. If $W$ on $(M, L)$ is the relative monodromy filtration, the following conditions are equivalent:

(1.9.1) $W$ on $\left(M^{\prime}, L\right)$ is the relative monodromy filtration and $\mathrm{Gr}_{k}^{L} M^{\prime}=\operatorname{Im}\left(\mathrm{Gr}_{k}^{L} u\right) \oplus \operatorname{Ker}\left(\mathrm{Gr}_{k}^{L} v\right)$ for any $k$,

$$
\begin{aligned}
& L_{k} M^{\prime}=u\left(L_{k} M\right)+\left(v^{-1}\left(S^{-1} L_{k} M\right) \cap W_{k} M^{\prime}\right) \text { for any } k, \\
& L_{k} M^{\prime}=v^{-1}\left(S^{-1} L_{k} M\right) \cap\left(u\left(L_{k} M\right)+W_{k} M^{\prime}\right) \text { for any } k .
\end{aligned}
$$

(Note that (1.9.1) is self-dual and (1.9.2-3) are dual to each other.)

Proof. The equivalence of (1.9.2-3) is clear. We show the equivalence of 
(1.9.1-2). We first verify that (1.9.2) is equivalent to:

$$
\begin{gathered}
W_{k} M^{\prime} \cap v^{-1}\left(S^{-1} L_{k} M\right)=W_{k} L_{k} M^{\prime} \\
u: L_{k} M \longrightarrow L_{k} M^{\prime} / W_{k} L_{k} M^{\prime} \text { is surjective. }
\end{gathered}
$$

(In fact, (1.9.2) implies (1.9.4), because $u\left(L_{k} M\right) \subset v^{-1}\left(S^{-1} L_{k} M\right)$.)

Then (1.9.4) is equivalent to

$$
W_{i} M^{\prime} \cap v^{-1}\left(S^{-1} L_{k} M\right)=W_{i} L_{k} M^{\prime} \quad \text { for } i \leq k,
$$

i.e. to the injectivity of

$$
v: W_{i} M^{\prime} / W_{i} L_{k} M^{\prime} \longrightarrow S^{-1}\left(W_{i-1} M / W_{i-1} L_{k} M\right) \quad \text { for } i \leq k .
$$

By the commutative diagram:

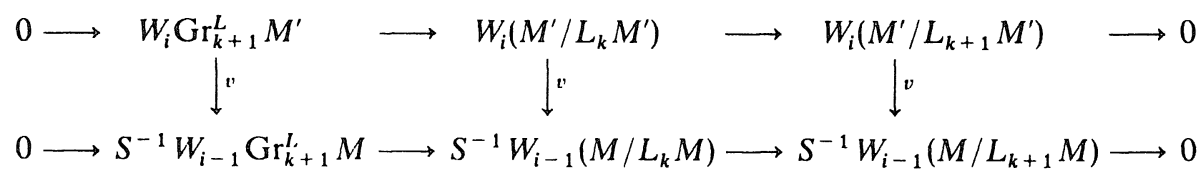

this condition is equivalent to the injectivity of

$$
v: W_{i} \mathrm{Gr}_{k}^{L} M^{\prime} \longrightarrow S^{-1} W_{i-1} \mathrm{Gr}_{k}^{L} M \quad \text { for } i<k .
$$

Similarly, (1.9.5) is equivalent to the surjectivity of

$$
u: \operatorname{Gr}_{k}^{L}\left(M / W_{i+1} M\right) \longrightarrow \operatorname{Gr}_{k}^{L}\left(M^{\prime} / W_{i} M^{\prime}\right) \quad \text { for } i \geq k .
$$

Therefore the assertion follows from 1.8.

1.10. Lemma. With the notation and the assumption of 1.5 , assume $\mathscr{C}$ is an abelian category. We define a filtration $L^{\prime}$ on $M$ by:

$$
L_{k}^{\prime} M=N\left(S L_{k} M\right)+W_{k-1} L_{k} M, \quad c f .(1.9 .2) .
$$

Then $W[1]$ is the relative monodromy filtration of $\left(M, L^{\prime}\right)$, the three filtrations $L, L^{\prime}, W$ are compatible, and the decomposition in 1.5:

$$
\mathrm{Gr}_{i}^{W} M=\sum_{j+k-2 m=i, 0 \leq m \leq j} \operatorname{Im} N^{m} s_{j+k, k}^{\prime}
$$

gives a bi-splitting of $L, L^{\prime}$ such that

$$
\mathrm{Gr}_{k}^{L} \mathrm{Gr}_{l}^{L^{\prime}} \mathrm{Gr}_{i}^{W} M \simeq \begin{cases}\sum_{j+k-2 m=i, 0<m \leq j} \operatorname{Im} N^{m} S_{j+k, k}^{\prime} & \text { if } l=k \\ \operatorname{Im} s_{i, k}^{\prime} & \text { if } l>k, i=l-1 \\ 0 & \text { otherwise. }\end{cases}
$$

Moreover the induced decomposition on $\mathrm{Gr}_{k}^{L^{\prime}} \mathrm{Gr}_{i}^{W} M$ is compatible with $\mathrm{Gr}_{i}^{W}$ of the 
canonical decomposition in (1.9.1):

$$
\mathrm{Gr}_{i}^{W} \mathrm{Gr}_{k}^{L^{\prime}} M=\mathrm{Gr}_{i}^{W} \operatorname{Im~Gr}_{k}^{L} u \oplus \mathrm{Gr}_{i}^{W} \operatorname{Ker}_{\mathrm{Gr}_{k}^{L^{\prime}}} v, \quad c f . \text { (1.8.7), }
$$

where $\mathrm{Gr}_{k}^{L} u: S \mathrm{Gr}_{k}^{L} M \rightarrow \mathrm{Gr}_{k}^{L^{\prime}} M$ and $\mathrm{Gr}_{k}^{L^{\prime}} v: \mathrm{Gr}_{k}^{L^{\prime}} M \rightarrow \mathrm{Gr}_{k}^{L} M$ are induced by $N$ and id respectively (and strictly compatible with $W$ ) and we have:

$$
\mathrm{Gr}_{i}^{W} \operatorname{Im} \mathrm{Gr}_{k}^{L} u \simeq \mathrm{Gr}_{k}^{L} \mathrm{Gr}_{k}^{L^{\prime}} \mathrm{Gr}_{i}^{W} M\left(=N\left(S \mathrm{Gr}_{k}^{L} \mathrm{Gr}_{i+2}^{W} M\right)\right)
$$

$$
\mathrm{Gr}_{i}^{W} \operatorname{Ker} \operatorname{Gr}_{l}^{L^{\prime}} v \simeq \begin{cases}\sum_{k} \operatorname{Im} s_{i, k}^{\prime} & \text { if } i=l-1 \\ 0 & \text { otherwise. }\end{cases}
$$

Proof. The first assertion is clear by 1.9. We verify as in the proof of 1.9:

$$
L_{l}^{\prime} W_{i} M= \begin{cases}L_{l} W_{i} M & \text { for } i<l \\ N\left(S L_{l} W_{i+2} M\right)+L_{l} W_{l-1} M & \text { for } i \geq l .\end{cases}
$$

In fact the assertion for $i \geq l$ is equivalent to the strictness of

$$
N:\left(L_{l} M / W_{l+1} L_{l} M, W\right) \longrightarrow S^{-1}\left(L_{l} M / W_{l-1} L_{l} M, W[2]\right)
$$

and follows from the injectivity of $N: \mathrm{Gr}_{i+2}^{W} \mathrm{Gr}_{k}^{L} M \rightarrow S^{-1} \mathrm{Gr}_{i}^{W} \mathrm{Gr}_{k}^{L} M$ for $i \geq k$. Similarly we verify

$$
\begin{aligned}
& N:\left(L_{k} \mathrm{Gr}_{i+2}^{W} M, L\right) \longrightarrow S^{-1}\left(L_{k} \mathrm{Gr}_{i}^{W} M, L\right) \text { is strictly injective } \\
& \text { for } k \leq i \text {. }
\end{aligned}
$$

Then (1.10.5-6) imply:

$$
L_{k} L_{l}^{\prime} \mathrm{Gr}_{i}^{W}= \begin{cases}L_{\min (k, l)} \mathrm{Gr}_{i}^{W} M & \text { for } i<l . \\ N\left(S L_{\min (k, l)} \mathrm{Gr}_{i+2}^{W} M\right) & \text { for } i \geq l .\end{cases}
$$

Therefore $L, L^{\prime}, W$ are compatible by $[19,(1.2 .14)]$, and we have:

$$
L_{l}^{\prime} \mathrm{Gr}_{k}^{L} \mathrm{Gr}_{i}^{W} M= \begin{cases}\mathrm{Gr}_{i}^{W} \mathrm{Gr}_{k}^{L} M & \text { for } i<l, k \leq l \\ N\left(S \mathrm{Gr}_{i+2}^{W} \mathrm{Gr}_{k}^{L} M\right) & \text { for } i \geq l, k \leq l \\ 0 & \text { otherwise, }\end{cases}
$$

$$
\mathrm{Gr}_{k}^{L} \mathrm{Gr}_{l}^{L^{\prime}} \mathrm{Gr}_{i}^{W} M= \begin{cases}N\left(\mathrm{SGr}_{i+2}^{W} \mathrm{Gr}_{k}^{L} M\right) & \text { for } k=l \\ P \mathrm{Gr}_{i}^{W} \mathrm{Gr}_{k}^{L} M & \text { for } k<l=i+1 \\ 0 & \text { otherwise, }\end{cases}
$$

by the Lefschetz decomposition (and the surjectivity or $N: \operatorname{Gr}_{i+2}^{W} \operatorname{Gr}_{k}^{L} M$ $\rightarrow S^{-1} \mathrm{Gr}_{i}^{W} \mathrm{Gr}_{k}^{L} M$ for $i<k$ ). Thus we get (1.10.3), and the inclusions $\supset$ in (1.10.4) are clear by the strictness of $\mathrm{Gr}_{k}^{L} u, \mathrm{Gr}_{k}^{L} v$ (cf. (1.8.2), (1.8.7)), then the equality follows from (1.10.3).

1.11. Remark. Let $M, M^{\prime}$ be objects of an abelian category with finite 
filtrations $L, W^{(1)}$ and a nilpotent endomorphism $N_{1}$ preserving $L$, such that $W^{(1)}$ on $M$ is the relative monodromy filtration for $L, N_{1}$. Let $u: M \rightarrow M^{\prime}$, $v: M^{\prime} \rightarrow M$ be morphisms compatible with $L, W^{(1)}$ and $N_{1}$, such that $N_{2}:=v u$ on $M$ and $N_{2}:=u v$ on $M^{\prime}$ are nilpotent. Put $M_{i}=\operatorname{Gr}_{i}^{L} M, M_{i}^{\prime}=\operatorname{Im} N_{2} \subset M_{i}$ with the morphisms $u_{i}: M_{i} \rightarrow M_{i}^{\prime}, v_{i}: M_{i}^{\prime} \rightarrow M_{i}$ induced by $N_{2}$ and the natural inclusion. Let $\tilde{W}^{(1)}, \tilde{W}^{(2)}, \tilde{W}^{(12)}$ be the monodromy filtration on $M_{i}, M_{i}^{\prime}$ for $N_{1}, N_{2}, N_{1}+N_{2}$ respectively. Assume:

(1.11.1) $\quad \mathrm{Gr}_{k}^{\tilde{W}^{(1)}} M_{i}^{\prime}=\operatorname{Im~Gr}_{k}^{\tilde{W}^{(1)}} u_{i} \oplus \operatorname{Ker~Gr}_{k}^{\tilde{W}^{(1)}} v_{i}$ for any $i, k$,

(1.11.2) $\quad \tilde{W}^{(12)}$ is the relative monodromy filtration on $M_{i}, M_{i}^{\prime}$ for $\tilde{W}^{(1)}, N_{2}$ and for $\tilde{W}^{(2)}, N_{1}$,

(1.11.3) the relative monodromy filtration $W^{(2)}$ for $L, N_{2}$ exists on $M, M^{\prime}$, (1.11.4) $\quad u W_{i}^{(2)} \subset W_{i-1}^{(2)}, v W_{i}^{(2)} \subset W_{i-1}^{(2)}$ and the equivalent conditions (1.9.1-3) are satisfied for

$$
\left(M ; L, W^{(2)}\right) \underset{v}{\stackrel{u}{\rightleftarrows}}\left(M^{\prime} ; L, W^{(2)}\right)
$$

(1.11.5) the relative monodromy filtration $W^{(12)}$ for $W^{(2)}, N_{1}$ exists on $M, M^{\prime}$,

(1.11.6) $L, W^{(1)}, W^{(12)}$ and $L, W^{(2)}, W^{(12)}$ are compatible three filtrations on $M, M^{\prime}$.

Then $W^{(12)}$ on $M, \mathrm{Gr}_{i}^{L} M$ is the relative monodromy filtration for $W^{(1)}, N_{2}$, and the following two conditions are equivalent:

(1.11.7) $W^{(1)}$ on $M^{\prime}$ is the relative monodromy filtration for $L, N_{1}$,

(1.11.8) $W^{(12)}$ on $M^{\prime}, \mathrm{Gr}_{i}^{L} M^{\prime}$ is the relative monodromy filtration for $W^{(1)}$, $N_{2}$ and the equivalent conditions (1.9.1-3) are satisfied for

$$
\begin{aligned}
& \left(M ; W^{(1)}, W^{(12)}\right) \underset{v}{\stackrel{u}{\rightleftarrows}}\left(M^{\prime} ; W^{(1)}, W^{(12)}\right) \\
& \left(\mathrm{Gr}_{i}^{L} M ; W^{(1)}, W^{(12)}\right) \underset{v}{\stackrel{u}{\rightleftarrows}}\left(\mathrm{Gr}_{i}^{L} M^{\prime} ; W^{(1)}, W^{(12)}\right) \text { for any } i .
\end{aligned}
$$

Here note that $u W_{i}^{(12)} \subset W_{i-1}^{(12)}, v W_{i}^{(12)} \subset W_{i-1}^{(12)}$ follow from (1.11.4) and the functoriality of the relative monodromy filtration. By (1.11.4) we have the decomposition

$$
\mathrm{Gr}_{i}^{L} M^{\prime}=\operatorname{Im~Gr}_{i}^{L} u \oplus \operatorname{Ker} \operatorname{Gr}_{i}^{L} v
$$

compatible with the action of $N_{1}, N_{2}$, and $\operatorname{Im~}_{\mathrm{Gr}_{i}^{L}}^{L} u$ is identified with $M_{i}^{\prime}$. Therefore the conditions (1.11.1-2) are satisfied by replacing $M_{i}, M_{i}^{\prime}, u_{i}, v_{i}$ 
with $\mathrm{Gr}_{i}^{L} M, \mathrm{Gr}_{i}^{L} M^{\prime}, \mathrm{Gr}_{i}^{L} u, \mathrm{Gr}_{i}^{L} v$ respectively. Then $W^{(12)}$ on $M, M^{\prime}$ is the relative monodromy filtration for $L, N_{1}+N_{2}$ by (1.11.2) and (1.11.6), because $N_{a} W_{i}^{(12)} \subset W_{i-2}^{(12)}(a=1,2)$ is clear and the canonical splitting 1.5 with the compatibility (1.11.6) implies

$$
N_{1}^{k}: \mathrm{Gr}_{j+k}^{W^{(12)}} \mathrm{Gr}_{j}^{W^{(2)}} \mathrm{Gr}_{i}^{L} M \longrightarrow \mathrm{Gr}_{j-k}^{W^{(12)}} \mathrm{Gr}_{j}^{W^{(2)}} \mathrm{Gr}_{i}^{L} M \text { (same for } M^{\prime} \text { ). }
$$

Similarly $W^{(12)}$ on $M, \mathrm{Gr}_{i}^{L} M$ is the relative monodromy filtration for $W^{(1)}, N_{2}$, and this holds for $M^{\prime}$ if (1.11.7) is satisfied. Therefore (1.11.7) implies (1.11.8), because it is enough to show the assertion for $\mathrm{Gr}_{i}^{L}$ using the canonical splitting of $L$ on $\mathrm{Gr}_{j}^{W^{(1)}}$ compatible with $u, v$ and $W^{(12)}$. Conversely (1.11.8) implies (1.11.7), because $N_{1} W_{i}^{(1)} M^{\prime} \subset W_{i-2}^{(1)} M^{\prime}$ follows from (1.9.2-3) and the assertion is reduced to that for $\mathrm{Gr}_{i}^{L}$ by the compatibility (1.11.6).

The above argument can be used to prove a result of Kashiwara $[13,5.5 .1]$ on the existence of the weight filtration of the open direct image of an admissible variation of mixed Hodge structure in the normal crossing case. In this case $\left(M, W^{(12)}\right),\left(M^{\prime}, W^{(12)}\right)$ underlie mixed Hodge structures, and $\left(\mathrm{Gr}_{i}^{L} M ; N_{1}, N_{2}\right)\left(\mathrm{Gr}_{i}^{L} M^{\prime} ; N_{1}, N_{2}\right)$ nilpotent orbits of weight $i-1, i$. Then the condition $(1.11 .1)$ is satisfied by $[8,1.16][15,2.1 .5]$ and $[19,5.2 .15]$ (cf. also $[13,5.6 .5])$, and $(1.11 .2)$ by $[6, \S 3]$. Here the compatibility condition (1.11.6) is trivial, because $W^{(1)}, W^{(2)}, L$ are filtrations in the abelian category of mixed Hodge structures and $W^{(12)}$ is the weight filtration. In this case we define the filtration $W^{(1)}$ on $M^{\prime}$ by $(1.9 .2-3)$. Then the condition (1.11.8) for $\mathrm{Gr}_{i}^{L}$ is satisfied by the functoriality of $(1.9 .2-3)$ and the strictness of $W^{(1)}$, cf. also 3.22 .

\section{§2. Mixed Hodge Modules on Complex Spaces}

\section{(2.a) Vanishing Cycle Functors and Specializations (Divisor Case)}

2.1. Let $X$ be a complex manifold, $\operatorname{MH}(X, n)^{(p)}$ the category of (polarizable) Hodge Modules (with $\mathbb{Q}$-structures) of weight $n$ (cf. $[19,(5.1 .6),(5.2 .10)]$ ), and MHW $(X)^{(p)}$ the category of (polarizable) $W$-filtered Hodge Modules, i.e. $\operatorname{MHW}(X)^{(p)}$ is the full subcategory of $\mathrm{MF}_{h} \mathrm{~W}\left(\mathscr{D}_{X}, \mathbb{Q}\right)$ such that $(M, F, K, W) \in \operatorname{MHW}(X)^{(p)}$ iff $\operatorname{Gr}_{i}^{W}(M, F, K) \in \mathrm{MH}(X, i)^{(p)}$ for any $i$, where $\mathrm{MF}_{h} \mathrm{~W}\left(\mathscr{D}_{X}, \mathbb{Q}\right)$ is the category of holonomic filtered $\mathscr{D}_{X}$-Modules with $\mathbb{Q}$ structure, endowed with a compatible (locally) finite increasing filtration: $(M, F, K, W)$, cf. $[19,(5.1 .14)]$. (Here $\operatorname{MHW}(X)^{(p)}$ means $\mathrm{MHW}(X)$ (resp. $\left.\operatorname{MHW}(X)^{p}\right)$.)

Let $X$ be a reduced separated complex analytic space, and $X=\cup U_{i}$ a locally finite open covering with closed immersions $U_{i} \rightarrow V_{i}$ where $V_{i}$ are smooth. Set $U_{I}=\bigcap_{i \in I} U_{i}, V_{I}=\Pi_{i \in I} V_{i}$, then $U_{I}$ is a closed subspace of $V_{I}$. Let $\operatorname{pr}_{I J}: V_{J} \rightarrow V_{I}$ be natural projections for $I \subset J$. By definition [19, (5.3.12)], 
an object $\mathscr{M}$ of $\operatorname{MH}(X, n)$ is $\left\{\mathscr{M}_{I} \in \mathrm{MH}\left(V_{I}, n\right)\right\}$ with isomorphisms :

$$
u_{I J}:\left(\operatorname{pr}_{I J}\right)_{*} \mathscr{M}_{J} \simeq M_{I} \text { on } V_{I} \backslash\left(U_{I} \backslash U_{J}\right)
$$

for $I \subset J$, such that supp $M_{I} \subset U_{I}$ and $u_{I K}=u_{I J}{ }^{\circ}\left(\operatorname{pr}_{I J}\right)_{*} u_{J K}$ on $V_{I} \backslash\left(U_{I} \backslash U_{K}\right)$. Here this definition is independent of the choice of $U_{i}, V_{i}$, because we have locally a section of $\operatorname{pr}_{I J}$ on a neighborhood of $U_{J}$ and the independence of the section follows from $[19,(3.2 .6)]$. In particular, for any open set $U$ with a closed immersion $U \rightarrow V$ such that $V$ smooth there exists uniquely a Hodge Module on $V$, which represents $\mathscr{M}$ on $V \supset U$ (called the local representative of $\mathscr{M}$ associated to $U \rightarrow V)$. We define $\operatorname{MHW}(X)$ similarly, where $\operatorname{Gr}_{p}^{F} M_{I}$ are assumed to be $\mathcal{O}_{U_{I}}$-Modules (cf. $[19,(2.1 .20)]$ ). Then for $\mathscr{M}=\left\{\mathscr{M}_{I}=\right.$ $\left.\left(M_{I}, F, K_{I}\right)\right\} \in \mathrm{MH}(X, n)$, there exists uniquely $K \in \operatorname{Perv}\left(\mathbf{Q}_{X}\right)$ such that $\left.K\right|_{U_{I}} \simeq K_{I}$ for any $I$ by $[1]$. Similarly $(K, W)$ exists globally for $\mathscr{M} \in \operatorname{MHW}(X)$. (Here $W$ is locally finite on $X$.) We say $\mathscr{M} \in \mathrm{MH}(X, n)$ is polarizable, if there is a pairing $S: K \otimes K \rightarrow a_{X}^{\prime} \mathbf{Q}(-n)$, called a polarization of $\mathscr{M}$, whose restriction to any local representative is a polarization in the sense of $[19,(5.2 .10)]$. We denote by $\mathrm{MH}(X, n)^{p}$ (or $\left.\mathrm{MHW}(X)^{p}\right)$ the full subcategory of polarizable objects. We say $\mathscr{M}$ has strict support $Z$, if the underlying perverse sheaf of $\mathscr{M}$ is an intersection complex with support $Z$ (and if $Z$ is irreducible).

2.2. Let $X$ be a complex manifold, and $D$ a (positive) divisor on $X$. Let $E$ be the line bundle associated to $D$, and $s_{0}$ (resp. $s_{1}$ ) the zero section (resp. the canonical section corresponding to the global section 1 of $\mathcal{O}_{X}(D)$ ). Put $E_{i}$ $=\operatorname{Im} s_{i}(i=0,1)$. Then, by definition, each (local) defining equation $g$ of $D$ determines a (local) trivialization: $E \simeq X \times \mathbb{C}$ such that $E_{0}$ (resp. $E_{1}$ ) corresponds to $X \times\{0\}$ (resp. Im $i_{g}$ ), where $i_{g}$ is the immersion by graph. Conversely each (local) trivialization of $E$ (as a line bundle) determines a (local) defining equation of $D$. Let $\mathscr{M}=(M, F, K, W) \in \operatorname{MHW}(X)$ and put $\tilde{\mathscr{M}}=(\tilde{M}, F, \tilde{K}, W)$ $=\left(s_{1}\right)_{*} \mathscr{M} \in \operatorname{MHW}(E)$. Then $\tilde{M}$ has the filtration $V$ along $E_{0}$, and if the condition:

(2.2.1) the three filtrations $F, W, V$ are compatible on $\tilde{M}$, cf. [19, (1.1.13)],

is satisfied, $(\tilde{M}, F)$ is quasi-unipotent and regular along $E_{0}$, i.e. $(M, F)$ is so along any (local) defining equation $D$ (cf. $[19, \S 3.2]$ ), because the assertion is verified for each $\operatorname{Gr}_{i}^{W}(\tilde{M}, F)$ by definition. If the condition (2.2.1) is satisfied, we define the specialization by

$$
\operatorname{Sp}_{D}(M, F, K)=\left(\operatorname{Gr}^{V}(\tilde{M}, F) \otimes_{\mathrm{Gr}^{V} \mathscr{D}}\left(\mathscr{D}_{E}, F\right), \operatorname{Sp}_{D} K\right),
$$

where $\operatorname{Gr}^{V}(\tilde{M}, F)=\oplus \operatorname{Gr}_{\alpha}^{V}(\tilde{M}, F)$ is a filtered graded $\operatorname{Gr}^{V}\left(\mathscr{D}_{E}, F\right)$-Module, $\mathrm{Sp}_{D} K$ is Verdier's specialization (cf. [23]) and we have the canonical isomorphism (cf. [12]):

$$
\operatorname{Dr}\left(\mathrm{Gr}^{V} \tilde{M} \otimes_{\mathrm{Gr}^{V} \mathscr{D}} \mathscr{D}_{E}\right) \simeq \mathrm{Sp}_{D} K \otimes \mathbb{C}
$$


Here we identify $E$ with the normal bundle of $E_{0} \simeq X$, and $\left.E\right|_{D}$ with the normal cone of $D$ in $X\left(\simeq E_{1}\right)$. We have the filtration $L=\operatorname{Sp}_{D} W$ on $\operatorname{Sp}_{D}(M, F, K)$ by:

$$
L_{i} \operatorname{Sp}_{D}(M, F, K)=\operatorname{Sp}_{D}\left(W_{i}(M, F), W_{i} K\right) \text {. }
$$

Let $T$ be the monodromy of $K, T=T_{s} T_{u}$ the Jordan decomposition and $N$ $=(2 \pi i)^{-1} \log T_{u}$. Then the action of $N$ corresponds to the action on $\mathrm{Gr}^{V} \tilde{M}$ defined by $s-\alpha$ on $\operatorname{Gr}_{\alpha}^{V} \tilde{M}$, where $s$ is the Euler vector field corresponding to the natural $\mathbb{C}^{*}$-action on the line bundle $E$, i.e. $s=\operatorname{Gr} t \partial_{t}$ in the notation of $[19, \S 3.1]$. Put

$$
\operatorname{Sp}_{D}^{1} K=\operatorname{Ker}\left(T_{s}-1: \operatorname{Sp}_{D} K \longrightarrow \operatorname{Sp}_{D} K\right),
$$

then $\operatorname{DR}\left(\left(\oplus_{\alpha \in \mathbf{Z}} \operatorname{Gr}_{\alpha}^{V} M\right) \otimes_{\mathrm{Gr}^{V} \mathscr{D}}\left(\mathscr{D}_{E}\right) \simeq \operatorname{Sp}_{\mathrm{D}}^{1} K \otimes \mathbb{C}\right.$ and we define

$$
\operatorname{Sp}_{D}^{1}(M, F, K)=\left(\left(\oplus_{\alpha \in \mathrm{Z}} \operatorname{Gr}_{\alpha}^{V}(M, F) \otimes_{\mathrm{Gr}^{V} \mathscr{D}}\left(\mathscr{D}_{E}, F\right), \operatorname{Sp}_{D}^{1} K\right)\right.
$$

Because $L_{i} \mathrm{Sp}_{D} K$ are stable by the action of $T$ and $L_{i} \mathrm{Gr}^{V}(M, F)$ are compatible with the decomposition $\operatorname{Gr}^{V}(M, F)=\oplus_{\alpha} \mathrm{Gr}^{V}(M, F)$, we have also the filtration $L=\operatorname{Sp}_{D}^{1} W$ on $\operatorname{Sp}_{D}^{1}(M, F, K)$. If $E$ is trivial, i.e. $D$ is a principal divisor defined by some function $g$ on $X, \mathrm{Sp}_{D} K$ is isomorphic to Deligne's total nearby cycle functor $\Psi_{g} K$ and $\operatorname{Gr}^{V}(\tilde{M}, F)$ have the structure of filtered $\mathscr{D}_{X}$-Modules (depending on $g$ ) so that $\mathbb{D R} \operatorname{Gr}_{\alpha}^{V} \tilde{M} \simeq \psi_{g, \mathrm{e}(\alpha)} K \otimes \mathbb{C}[-1]$ or $\phi_{g, 1} K \otimes \mathbb{C}[-1]$, cf. $[19,(3.4 .12)]$ (because the choice of $g$ determines the trivialization: $E \simeq X$ $\times \mathbb{C}$ and the isomorphism: $\mathrm{Gr}_{0}^{V} \mathscr{D}_{E} \simeq \mathscr{D}_{X}[\mathrm{~s}]$.) In this case, we define

$$
\begin{aligned}
& \psi_{g}(M, F, K)=\left(\underset{-1 \leq \alpha<0}{\bigoplus_{1}} \operatorname{Gr}_{\alpha}^{V}(\tilde{M}, F[1]),{ }^{p} \psi_{g} K\right) \\
& \phi_{g, 1}(M, F, K)=\left(\operatorname{Gr}_{0}^{V}(\tilde{M}, F),{ }^{p} \phi_{g, 1} K\right)
\end{aligned}
$$

where ${ }^{p} \psi=\psi[-1]$, etc. We also define the filtration $L$ on $\psi_{g}(M, F, K)$ and $\phi_{g, 1}(M, F, K)$ by

$$
\begin{aligned}
& L_{i} \psi_{g}(M, F, K)=\psi_{g}\left(W_{i+1}(M, F, K)\right) \\
& L_{i} \phi_{g, 1}(M, F, K)=\phi_{g, 1}\left(W_{i}(M, F, K)\right) .
\end{aligned}
$$

Here the condition (2.2.1) is always assumed. Note that $\left(\operatorname{Sp}_{D}(M, F, K), L\right)$ is equivalent to $\left(\psi_{g}(M, F, K), \phi_{g, 1}(M, F, K) ; L\right)$, and the shift of the filtrations $F$ and $L$ on $\psi_{g}(M, K)$ comes from the external products with $\left(\Omega_{\mathrm{C}}^{1}, F, \mathbb{Q}_{\mathrm{C}}[1], W\right)$ (in the non characteristic case), where $\operatorname{Gr}_{i}^{F} \Omega_{\mathrm{c}}^{1}=0(i \neq-1)$ and $\operatorname{Gr}_{i}^{W}\left(\Omega_{\mathrm{C}}^{1}, \mathbb{Q}_{\mathrm{c}}[1]\right)$ $=0(i \neq 1)$.

If $X$ is not smooth, we assume $X$ is reduced separated and $D$ is a positive locally principal divisor. Then the normal cone of $D$ in $X$ is a closed subspace of the line bundle $E$ associated to $D$ on $X$ (by identifying $X$ with $\left.E_{1}\right)$. Therefore $\operatorname{Sp}_{D}^{(1)} K \in \operatorname{Perv}\left(Q_{E}\right), L$ and the action of $N$ are globally well- 
defined. Put $\tilde{\mathscr{M}}=\left(s_{1}\right)_{*} \mathscr{M}$ as above. We say that the condition (2.2.1) is satisfied for $\tilde{\mathscr{M}}$ if the following condition is satisfied:

there is an open covering $X=U_{i} U_{i}$ with $U_{i} \rightarrow V_{i}$ as in 2.1, such that $\left.E\right|_{U_{1}}$ are trivial (hence $\left.E\right|_{U_{\imath}} \hookrightarrow V_{i} \times \mathbf{C}$ ) and the condition (2.2.1) is satisfied for the represent $\tilde{\mathscr{M}}_{i}$ of $\tilde{\mathscr{M}}$ on $V_{i} \times \mathbf{C}$ (and for the filtration $V$ along $\left.V_{i} \times\{0\}\right)$ for any $i$.

This condition is independent of the trivialization $\left.E\right|_{U_{i}} \simeq U_{i} \times \mathbf{C}$ and the choice of $U_{i}, V_{i}$. From now on, we shall always assume this condition. If $E$ is trivial and $D=g^{-1}(0)$, we can define $\operatorname{Sp}_{D}^{(1)}(M, F, K), \psi_{g}(M, F, K), \phi_{g, 1}(M, F, K)$ and $L$, using $U_{i}, V_{i}$ as above, where we use the covering: $E=\cup_{i} U_{i} \times \mathbb{C}$ and the embeddings $U_{I} \times \mathbb{C} \rightarrow V_{I} \times \mathbb{C}$. We can also define $\operatorname{Sp}_{D}(M, F, K)$ and $L$, even if $E$ is not trivial.

2.3. Let $X$ be a separated reduced complex analytic space, and $D$ a (positive) locally principal divisor on $X$. We say that the specialization along $D$ is welldefined for $\mathscr{M} \in \mathrm{MHW}(X)$, if the condition (2.2.8) and the following condition are satisfied for $\mathscr{M}=((M, F), K ; W)$ :

(2.3.1) the relative monodromy filtration $W$ (with respect to the action of $N$ ) exists on $\left(\mathrm{Sp}_{D} K, L\right)$, cf. 1.1 .

Note that this condition is local (by the uniqueness of $W$ ) and (2.3.1) is equivalent to the existance of $W$ on $\left(\mathrm{Sp}_{D} K \otimes \mathbb{C}, L\right)$ or on $\left(\mathrm{Gr}^{V} \tilde{M}_{i}, L\right)$ for any $i$ (cf. (2.2.8)) by 1.2. In this case we define

$$
\mathrm{Sp}_{D}^{(1)} M=\left(\operatorname{Sp}_{D}^{(1)}((M, F), K), W\right) .
$$

If $D=g^{-1}(0)$, the condition (2.3.1) is equivalent to:

(2.3.3) the relative monodromy filtration $W$ exists on $\left({ }^{p} \psi_{g} K, L\right),\left({ }^{p} \phi_{g, 1} K, L\right)$, cf. (2.2.6-7),

and we say that the vanishing cycle functors along $g$ are well-defined for $\mathscr{M} \in \operatorname{MHW}(X)$, if the conditions (2.2.8) and (2.3.3) are satisfied. In this case we define:

$$
\psi_{g} \mathscr{M}=\left(\psi_{g}(M, F, K), W\right), \quad \phi_{g, 1} \mathscr{M}=\left(\phi_{g, 1}(M, F, K), W\right) .
$$

The following proposition gives a generalization of $[22,(4.11),(A .9)]$ and was pointed out by Kashiwara (cf. [9]). Note that the conditions (2.4.1), (2.4.3-4) were a part of definition in [21] and we have a non canonical splitting (2.4.2) in the polarizable case.

2.4. Proposition. Let $X$ be as above, $g$ a holomorphic function on $X$ and $\mathscr{M}$ $=((M, F), K, W) \in \mathrm{MHW}(X)\left(\right.$ resp. MHW $\left.(X)^{p}\right)$. Assume the vanishing cycle functors along $g$ are well-defined for $M$. Then: 
(2.4.1) the filtration $W$ in (2.3.3) induces the relative monodromy filtration on $\left(\psi_{g}(M, F, K), L\right),\left(\phi_{g, 1}(M, F, K), L\right)$ (i.e. $F, W, L$ are compatible on $\psi_{g} M, \phi_{g, 1} M$ and the monodromy filtration exists on $\operatorname{Gr}_{i}^{L} \psi_{g}(M, F)$, $\mathrm{Gr}_{i}^{L} \phi_{g, 1}(M, F)$, cf. 1.4),

(2.4.2) there is a canonical splitting of $L$ on $\operatorname{Gr}_{i}^{W} \psi_{g}(M, F, K)$, $\mathrm{Gr}_{i}^{W} \phi_{g, 1}(M, F, K)$,

(2.4.3) $\quad \psi_{g} M, \phi_{g, 1} \mathscr{M} \in \mathrm{MHW}(X)\left(\right.$ resp. $\left.\mathrm{MHW}(X)^{p}\right)$ and L induces a filtration of

$\psi_{g} \mathscr{M}, \phi_{g, 1} \mathscr{M}$ in $\operatorname{MHW}(X)$ (resp. $\left.\operatorname{MHW}(X)^{p}\right)$,

(2.4.4) can and Var induce morphisms in $\operatorname{MHW}(X)$ :

can $: \psi_{g, 1} \mathscr{M} \longrightarrow \phi_{g, 1} \mathscr{M}, \quad \operatorname{Var}: \phi_{g, 1} \mathscr{M} \longrightarrow \psi_{g, 1} \mathscr{M}(-1)$,

i.e. $\operatorname{can}\left(W_{i}^{p} \psi_{g, 1} K\right) \subset W_{i}^{p} \phi_{g, 1} K, \operatorname{Var}\left(W_{i}^{p} \phi_{g, 1} K\right) \subset W_{i-2}^{p} \psi_{g, 1} K(-1)$.

Proof. By the condition (2.2.1) we have the canonical isomorphisms:

$$
\begin{aligned}
& \operatorname{Gr}_{k}^{L} \psi_{g}(M, F, K) \simeq \psi_{g} \mathrm{Gr}_{k+1}^{W}(M, F, K), \\
& \operatorname{Gr}_{k}^{L} \phi_{g, 1}(M, F, K) \simeq \phi_{g, 1} \operatorname{Gr}_{k}^{W}(M, F, K)
\end{aligned}
$$

Therefore the monodromy filtration $W$ (shifted by $k$ ) exists on $\operatorname{Gr}_{k}^{L} \psi_{g}(M, F, K)$, etc. so that

$$
\left(\operatorname{Gr}_{k}^{L} \psi_{g}(M, F, K), W\right),\left(\operatorname{Gr}_{k}^{L} \phi_{g, 1}(M, F, K), W\right) \in \operatorname{MHW}(X)^{p},
$$

and (2.4.3) follows from (2.4.1-2). By 1.5 we have the canonical splitting of $L$ on the $\mathbb{Q}$-part $\operatorname{Gr}_{i}^{W}{ }^{p} \psi_{g} K$, etc., therefore for the proof of (2.4.2), it is enough to show its compatibility with the Hodge filtration, and the assertion is local by the uniqueness of the splitting. Thus we may assume $X$ is smooth, and the assertion follows from 1.5 applied to $\left(\oplus_{p} F_{p} \psi_{g} M, L\right)$. The remaining assertions (2.4.1)(2.4.4) are also local, and we may assume $X$ smooth. Then (2.4.4) follows from 1.7. We prove the restriction of (2.4.1) to $\left(L_{k}\left(M^{\prime}, F\right), L\right)$ by induction on $k$, where $\left(M^{\prime}, F\right)=\psi_{g}(M, F)$ or $\phi_{g, 1}(M, F)$. By 1.2 and 1.4 , it is enough to show:

$$
N^{i+1} F_{p} L_{k} M^{\prime} \cap L_{k-1} M^{\prime} \subset N^{i+1} F_{p} L_{k-1} M^{\prime}+F_{p+i+1} W_{-i-2+k} L_{k-1} M^{\prime},
$$

because $\mathrm{Gr}^{W} \mathrm{Gr}^{L} N^{j}$ are strict by $[19,(5.1 .14)]$. But the left hand side is contained in

$$
F_{p+i+1} L_{k-1} M^{\prime} \cap\left(N^{i+1} L_{k-1} M^{\prime}+W_{-i-2+k} L_{k-1} M^{\prime}\right)
$$

by 1.2 and (2.3.1). Therefore the assertion follows from the strictness of the morphism : 
$\left(N^{i+1}, \mathrm{id}\right):\left(L_{k-1} M^{\prime}, F[i+1]\right) \oplus\left(W_{-i-2+k} L_{k-1} M^{\prime}, F\right) \longrightarrow\left(L_{k-1} M^{\prime}, F\right)$,

cf. [loc. cit], because $\left(L_{k-1} M^{\prime}, F\right)$, etc. and $N^{i+1}$, etc. underlie objects and morphisms in $\mathrm{MHW}(X)$ by inductive hypothesis.

2.5. Proposition. Let $X$ be as in $2.4, \mathscr{M} \in \mathrm{MHW}(X)$, and $\mathscr{M}^{\prime}$ its subquotient in $\operatorname{MHW}(X)$. Then $\mathscr{M}^{\prime}$ is polarizable, if so is $\mathscr{M}$. Let $D$ be a (positive) locally principal divisor. Then the specialization along $D$ is well-defined for $\mathscr{M}^{\prime}$, if it holds for $\mathscr{M}$. Moreover, for an exact sequence in $\operatorname{MHW}(X)$ :

$$
0 \longrightarrow \mathscr{M}^{\prime} \longrightarrow \mathscr{M} \longrightarrow \mathscr{M}^{\prime \prime} \longrightarrow 0
$$

we have the canonical (and functorial) exact sequence in $\mathrm{MHW}(X)$ :

$$
\left.0 \longrightarrow \psi_{g} \mathscr{M}^{\prime} \longrightarrow \psi_{g} \mathscr{M} \longrightarrow \psi_{g} \mathscr{M}^{\prime \prime} \longrightarrow 0 \quad \text { (same for } \phi_{g, 1}\right) \text {, }
$$

if $D=g^{-1}(0)$ and the vanishing cycle functors along $g$ are well-defined for $\mathscr{M}$.

Proof. The first assertion follows from [19, 5.2.13] and the exactness of the functors $\mathrm{Gr}_{i}^{W}$ (cf. $[19,5.1 .14]$ ). (In fact, $\mathrm{Gr}_{i}^{W}$ of $(2.5 .1)$ splits by the polarization on $\mathrm{Gr}_{i}^{W} \mathscr{M}$ so that $\mathrm{Gr}_{i}^{W} \mathscr{M}^{\prime}$ and $\mathrm{Gr}_{i}^{W} \mathscr{M}^{\prime \prime}$ are polarizable.)

For the other assertions we may assume $X$ is smooth and $D$ $=g^{-1}(0)$. Taking the direct image $\left(s_{1}\right)_{*}$ (cf. 2.2), we may assume (2.5.1) is an exact sequence in $\mathrm{MHW}(E)$. Then by $[19,(3.1 .5)]$ the underlying $\mathscr{D}_{E}$-Modules $M^{\prime}, M, M^{\prime \prime}$ has the filtration $V$ along $E_{0}(\simeq X)$ and

$$
0 \longrightarrow\left(M^{\prime} ; F, W, V\right) \longrightarrow(M ; F, W, V) \longrightarrow\left(M^{\prime \prime} ; F, W, V\right) \longrightarrow 0
$$

is separately exact for $F, W, V$. By the proof of $[19,(5.1 .14)]$,

$$
0 \longrightarrow \operatorname{Gr}_{i}^{W}\left(M^{\prime} ; F, V\right) \longrightarrow \operatorname{Gr}_{i}^{W}(M ; F, V) \longrightarrow \operatorname{Gr}_{i}^{W}\left(M^{\prime \prime} ; F, V\right) \longrightarrow 0
$$

is exact (i.e. $F_{p} V_{\alpha} \mathrm{Gr}_{i}^{W}(2.5 .3)$ are exact). Using the commutative diagram:

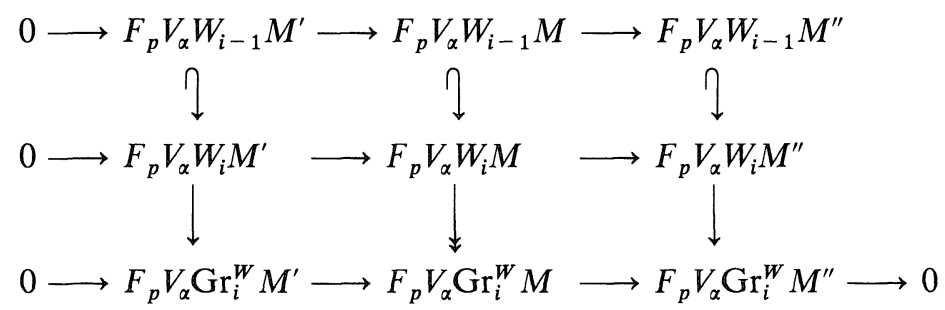

we verify by induction on $i$ that $F_{p} V_{\alpha} W_{i} M^{\prime \prime} \rightarrow F_{p} V_{\alpha} \mathrm{Gr}_{i}^{W} M^{\prime \prime}$ are surjective, i.e. $F, V, W$ are compatible on $M^{\prime \prime}$ (cf. $[19,(1.2 .14)]$ ), and $F_{p} V_{\alpha} W_{i} M \rightarrow F_{p} V_{\alpha} W_{i} M^{\prime \prime}$ are surjective, i.e. $(M ; F, V, W) \rightarrow\left(M^{\prime \prime} ; F, V, W\right)$ is a strict epimorphism. Therefore $\left(M^{\prime} ; F, W, V\right)$ must be the kernel of this strict epimorphism by the separate exactness, and $F, W, V$ are compatible on $M^{\prime}$. We now show the condition (2.3.1) for $M^{\prime \prime}$ in (2.5.4), because the assertion for $M^{\prime}$ follows from the strict 
surjectivity of

$$
(\tilde{K} ; L, W) \longrightarrow\left(\tilde{K}^{\prime \prime} ; L, W\right)
$$

where $\tilde{K}={ }^{p} \psi_{g} K,{ }^{p} \phi_{g, 1} K$ (same for $\tilde{K}^{\prime \prime}$ ). (Here the surjectivity of $\operatorname{Gr}_{i}^{W} \operatorname{Gr}_{k}^{L}(2.5 .4)$ follows from $[19,(5.1 .14)]$, and the strict surjectivity of $(2.5 .4)$ implies that the kernel of (2.5.4) gives the relative monodromy filtration on $\left(\tilde{K}^{\prime}, L\right)$.) Let $W$ be the monodromy filtration on $\mathrm{Gr}_{k}^{L} \widetilde{K}^{\prime \prime}$ shifted by $k$, then by [loc. cit],

$$
\mathrm{Gr}_{k}^{L} N^{i+1}:\left(\mathrm{Gr}_{k}^{L} \tilde{K}^{(\prime \prime)}, W\right) \longrightarrow\left(\mathrm{Gr}_{k}^{L} \tilde{K}^{(\prime \prime)}, W[2 i+2]\right)
$$

is strict and $\mathrm{Gr}^{W} \operatorname{Ker}\left(\mathrm{Gr}_{k}^{L} N^{i+1}\right) \simeq \operatorname{Ker}\left(\mathrm{Gr}^{W} \mathrm{Gr}_{k}^{L} N^{i+1}\right)$. Therefore

$$
\operatorname{Ker}\left(N^{i+1}: \operatorname{Gr}_{k}^{L} \tilde{K} \longrightarrow \operatorname{Gr}_{k}^{L} \tilde{K}\right) \longrightarrow \operatorname{Ker}\left(N^{i+1}: \operatorname{Gr}_{k}^{L} \widetilde{K}^{\prime \prime} \longrightarrow \operatorname{Gr}_{k}^{L} \widetilde{K}^{\prime \prime}\right)
$$

is surjective by Lefschetz decomposition. Thus we get the assertion by 1.2.

2.6. Proposition. Let $X$ be as in 2.4 and $\mathscr{M}=(M, F, K, W) \in \mathrm{MHW}(X)$, where $(M ; F, W):=\left\{\left(M_{I} ; F, W\right)\right\}$, cf. 2.1. We define

$$
\mathbf{D} \mathscr{M}=(\mathbf{D}(M ; F, W), \mathbf{D}(K, W))
$$

by $\mathbb{D}(M ; F, W)=\left\{\mathbf{D}\left(M_{I} ; F, W\right)\right\}$ so that

$$
W_{i}(\mathbf{D} \mathscr{M})=\mathbb{D}\left(\mathscr{M} / W_{-i-1} \mathscr{M}\right), \mathrm{Gr}_{i}^{W} \mathbf{D} \mathscr{M}=\mathbf{D G r}_{-i}^{W} \mathscr{M} .
$$

Then $\mathbb{D} \mathscr{M} \in \operatorname{MHW}(X)$ (it is independent of the choice of $U_{i}, V_{i}$ ), and it is polarizable if so is $\mathscr{M}$. Let $g$ be a holomorphic function on $X$, then the vanishing cycle functors along $g$ are well-defined for $\mathscr{M}$ iff it holds for $\mathbf{D} \mathscr{M}$; in this case we have the canonical isomorphisms:

$$
\psi_{g} \mathbf{D} \mathscr{M} \stackrel{\sim}{\longrightarrow}\left(\mathbf{D} \psi_{g} \mathscr{M}\right)(1), \quad \phi_{g, 1} \mathbf{D} \mathscr{M} \stackrel{\sim}{\longrightarrow} \mathbf{D} \phi_{g, 1} \mathscr{M}
$$

Proof. By the compatibility of topological and analytic dualities for closed immersions of complex manifolds, we see that $\mathbb{D} \mathscr{M}$ is well-defined (i.e. independent of $\left.U_{i}, V_{i}\right)$. Then the second isomorphism in (2.6.1) implies that $\operatorname{MHW}(X)^{p}$ is stable by $\mathbb{D}$. For the stability of $\operatorname{MHW}(X)$, we may assume $X$ is smooth, then the assertion follows from the compatibility of topological and analytic dualities for the vanishing cycle functors (see [25]):

$$
\begin{aligned}
& { }^{p} \psi_{g} \mathrm{D} K \stackrel{\sim}{\longrightarrow}\left(\mathbb{D}^{p} \psi_{g} K\right)(1),{ }^{p} \phi_{g, 1} \mathrm{D} K \stackrel{\sim}{\rightarrow} \mathbb{D}^{p} \phi_{g, 1} K, \\
& \psi_{g} \mathrm{D}(M, F) \stackrel{\sim}{\longrightarrow} \mathrm{D} \psi_{g}(M, F)(1), \phi_{g, 1} \mathbb{D}(M, F) \stackrel{\sim}{\longrightarrow} \phi_{g, 1}(M, F),
\end{aligned}
$$

where the morphism in (2.6.3) are defined in [19, (5.2.3)]. In fact, we can verify inductively the condition of Hodge Modules of $\mathrm{Gr}_{i}^{W} \mathrm{D} \mathscr{M}$ (for example, the stability of the regularity and the quasi-unipotency along $g$ by $\mathbf{D}$ follows from the proof of $[19,(5.1 .13)]$.) Here note that the (relative) monodromy filtration is self-dual. Therefore it remains to show the stability of the condition (2.2.1) 
by $\mathbb{D}$ (because (2.3.1) follows from the above remark). Put $(\tilde{M}, F, W)$ $=\left(i_{g}\right)_{*}(M, F, W)$, where we may assume $X$ is smooth. We take locally a free resolution as in $[19,(5.1 .13)]$ :

$$
(L ; F, V, W) \longrightarrow(\tilde{M} ; F, W, V)
$$

such that each $L^{j}$ has a splitting of $W$ compatible with $F, V$ (hence $F, V, W$ are compatible) and $\operatorname{Gr}_{i}^{W}\left(L^{j}, F, V\right)$ are finite direct sums of $(\mathscr{D}, F[p], V[\alpha])$ $(-1 \leq \alpha \leq 0)$. Here note that a filtered morphism: $(M ; F, V, W)$ $\rightarrow\left(M^{\prime \prime} ; F, V, W\right)$ is strictly surjective iff $F_{p} V_{\alpha} W_{i} M \rightarrow F_{p} V_{\alpha} W_{i} M^{\prime \prime}$ are surjective for any $p, \alpha, i$ (this is not true for a strict monomorphism), cf. $[19, \S 1.2]$. We define $\mathbb{D}(L ; F, V, W)$ by $(2.6 .1)$ (for $W)$ and

$$
\mathbb{D}(\mathscr{D}, F[p], V[\alpha])=(\omega \otimes \mathscr{D}, F[-p], V[-1-\alpha])\left[d_{X}\right] .
$$

Then $\mathrm{Gr}_{i}^{W} \mathrm{D}(L ; F, V)$ is strict by [loc. cit], and $\mathscr{H}^{j} \mathrm{Gr}_{i}^{W} \mathbf{D}(L ; F, V)=0$ for $j \neq 0$ by the holonomicity of $\tilde{M}$. Therefore $\mathbb{D}(L ; F, V, W)$ is strict and $F, V, W$ are compatible on $\mathscr{H}^{0} \mathrm{D} L$ by $[19,(1.2 .9)]$. By definition and by the proof of $[19,(5.1 .13)]$ we have the isomorphism: $\mathbf{D}(M ; F, V, W)=\mathscr{H}^{0} \mathbf{D}(L ; F, V, W)$. Thus we get the assertion.

\section{(2.b) Extensions over Locally Principal Divisors}

2.7. Let $X$ be a complex manifold, put $E=X \times \mathbf{C}, E^{*}=X \times \mathbb{C}^{*}, E_{0}=X \times\{0\}$ and let $j: E^{*} \rightarrow E$ be the natural inclusion. If $\mathscr{M}^{\prime}=\left(\left(M^{\prime}, F\right), K^{\prime} ; W\right) \in \operatorname{MHW}\left(E^{*}\right)$ is extended to $\mathscr{M}=((M, F), K ; W) \in \operatorname{MHW}(E)$ (i.e. $\left.j^{-1} \mathscr{M}=\mathscr{M}^{\prime}\right)$ such that the specialization along $E_{0}$ is well-defined for $\mathscr{M}$, the following conditions are satisfied :

(2.7.1) $\quad j_{*} K^{\prime}$ (or equivalently $j_{!} K^{\prime}$ ) is cohomologically constructible (i.e. $j_{*} K^{\prime}, j_{!} K^{\prime} \in \operatorname{Perv}\left(\mathbf{Q}_{E}\right)$ ),

(2.7.2) $\quad F_{p} V_{<0} M=V_{<0} M \cap j_{*} F_{p} M^{\prime}$ are coherent over $\mathcal{O}_{E}$,

(2.7.3) $F, V, W$ are compatible on $V_{<0} M$,

(2.7.4) the relative monodromy filtration exists on $\left(\psi_{t} K, L\right)$,

(2.7.5) $\operatorname{Gr}_{i}^{W} \mathscr{M}^{\prime}$ are extended to Hodge Modules on E.

If moreover $\mathscr{M}$ is polarizable, we have

$\mathrm{Gr}_{i}^{W} \mathscr{M}^{\prime}$ are extended to polarizable Hodge Modules on $E$.

Note that $\left(V_{<0} M ; F, W, V\right)$ depends only on $j^{-1} \mathscr{M}=\mathscr{M}^{\prime}$ (independent of the extension $\mathscr{M})$, cf. [12] [19, (13.1.7)] for $V_{<0} M$, and the conditions (2.7.1-6) are concerning only $\mathscr{M}^{\prime}$. (We shall see later that $\mathscr{M}^{\prime}$ is extended if these 
conditions are satisfied.) If (2.7.1-5)(resp. (2.7.1-6)) are satisfied,

$$
\psi_{t} \mathscr{M}:=\left(\oplus{ }_{-1 \leq \alpha<0} \operatorname{Gr}_{\alpha}^{V}(M, F[1]),{ }^{p} \psi_{t} K ; W\right)
$$

(cf. (2.2.6) (2.3.4)) belongs to $\mathrm{MHW}\left(E_{0}\right)$ (resp. $\left.\mathrm{MHW}\left(E_{0}\right)^{p}\right)$ by the same argument as in the proof of 2.4 , and $\psi_{t} \mathscr{M}$ will be sometimes denoted by $\psi_{t} \mathscr{M}^{\prime}$, because it depends only on $\mathscr{M}^{\prime}=j^{-1} \mathscr{M}$.

If $X$ is singular and $\mathscr{M}^{\prime}$ is extended to $\mathscr{M}$ so that the specialization along $E_{0}$ is well-defined, the conditions (2.7.1-5)(resp. (2.7.1-6)) are satisfied for the representative of $\mathscr{M}^{\prime}$ on $V \times \mathbb{C}^{*}$ associated to any closed immersion $U \rightarrow V$ as in 2.1. Then we can define $\psi_{t} \mathscr{M}^{\prime} \in \operatorname{MHW}\left(E_{0}\right)^{(p)}$ similarly. Let $\operatorname{MHW}\left(E^{*}\right)_{e x}^{(p)}$ be the full subcategory of $\mathrm{MHW}\left(E^{*}\right)^{(p)}$ whose objects are satisfying the conditions (2.7.1-5) (or (2.7.1-6)), i.e. their local representatives as above satisfy the conditions. Let $\operatorname{MHW}\left(E^{*}, E_{0}\right)_{e x}^{(p)}$ be the category whose objects are $\left(\mathscr{M}^{\prime}, \mathscr{M}^{\prime \prime}, u, v\right) \quad$ where $\quad \mathscr{M}^{\prime} \in \operatorname{MHW}\left(E^{*}\right)_{\text {ex }}^{(p)}, \mathscr{M}^{\prime \prime} \in \mathrm{MHW}\left(E_{0}\right)^{(p)}$ and $u: \psi_{t, 1} \mathscr{M}^{\prime}$ $\rightarrow \mathscr{M}^{\prime \prime}, v: \mathscr{M}^{\prime \prime} \rightarrow \psi_{t, 1} \mathscr{M}^{\prime}(-1)$ are morphisms in $\operatorname{MHW}\left(E_{0}\right)$ such that $v u$ $=N$. Here the morphisms are the pairs of morphisms in $\operatorname{MHW}\left(E^{*}\right), \operatorname{MHW}\left(E_{0}\right)$ compatible with $u, v$. Finally, let $\operatorname{MHW}(E)_{s p}^{(p)}$ be the full subcategory of $\mathrm{MHW}(E)^{(p)}$ defined by the condition: the specializations along $E_{0}$ is well defined. Then we have a natural functor

$$
\operatorname{MHW}(E)_{s p}^{(p)} \longrightarrow \operatorname{MHW}\left(E^{*}, E_{0}\right)_{e x}^{(p)}
$$

which assigns $\left(j^{-1} \mathscr{M}, \phi_{t, 1} \mathscr{M}\right.$, can, Var $)$ to $\mathscr{M}$.

2.8. Proposition. The functor (2.7.7) is an equivalence of categories.

Proof. We first assume $X$ smooth. Let $\left(\mathscr{M}^{\prime}, \mathscr{M}^{\prime \prime}, u, v\right) \in \mathrm{MHW}\left(E^{*}, E_{0}\right)_{e x}^{(p)}$. By Deligne-MacPherson-Verdier's theory on extensions of perverse sheaves (cf. for example [23]) and by [12] [14][18][19, (3.4.12)], there exists uniquely $(M, K)$ with isomorphisms :

$$
j^{-1}(M, K) \simeq\left(M^{\prime}, K^{\prime}\right), \phi_{t, 1}(M, K) \simeq\left(M^{\prime \prime}, K^{\prime \prime}\right)
$$

such that can, Var are identified with the underlying morphisms of $u, v$. We define the filtration $F$ on $V_{0} M$ by

$$
m \in F_{p} V_{0} M \Leftrightarrow j^{*} m \in F_{p} M^{\prime}, \mathrm{Gr}_{0}^{V} m \in F_{p} M^{\prime \prime},
$$

where we use (2.8.1). Then $\left(V_{0} M, F\right) \rightarrow\left(M^{\prime \prime}, F\right)$ is surjective (i.e. $\operatorname{Gr}_{0}^{V}(M, F)$ $\left.\simeq\left(M^{\prime \prime}, F\right)\right)$, because for $m \in V_{0} M$ such that $\operatorname{Gr}_{0}^{V} m \in F_{p} M^{\prime \prime}$, we have $\operatorname{Gr}_{-1}^{V}(m t) \in$ $F_{p} \mathrm{Gr}_{-1}^{V} M$ and $m t=m^{\prime}+m^{\prime \prime} t$ with $m^{\prime} \in F_{p} V_{-1} M, m^{\prime \prime} \in V_{<0} M$, i.e. $m-m^{\prime \prime} \in F_{p} V_{0} M$. We define

$$
F_{p} M=\sum_{i}\left(F_{p-i} V_{0} M\right) \partial_{t}^{i}
$$

Then the induced filtration $F$ on $V_{0} M$ coincides with the original one and $(M, F)$ 
is quasi-unipotent and regular along $E_{0}(\mathrm{cf} .[19, \S 3.2])$, because

$$
V_{0} M \cap\left(F_{p} V_{0} M\right) \partial_{t}=V_{0} M \cap\left(F_{p} V_{-1} M\right) \partial_{t} \subset F_{p+1} V_{0} M
$$

(cf. the proof of $[19,(3.2 .2)]$ ). Then, by (2.7.2), $F_{p} V_{\alpha} M$ and $F_{p} M$ are coherent over $\mathcal{O}_{E}$, and $(M, F)$ is a coherent filtered $\mathscr{D}_{E}$-Module, because $\left(V_{0} M, F\right)$ is coherent over $\left(V_{0} \mathscr{D}_{E}, F\right)$. We now define the filtration $L$ on $\phi_{t, 1}(M, K)$ by

$$
\begin{aligned}
L_{k} \phi_{t, 1}(M, K)= & \operatorname{can}\left(L_{k-1} \psi_{t, 1}(M, K)\right) \\
& +\left(\operatorname{Var}^{-1}\left(L_{k-1} \psi_{t, 1}(M, K)\right) \cap W_{k} \phi_{t, 1}(M, K)\right)
\end{aligned}
$$

where $L_{k-1} \psi_{t, 1}(M, K)=\psi_{t, 1} W_{k}\left(M^{\prime}, K^{\prime}\right)$ and $W$ on $\phi_{t, 1}(M, K)$ is the weight filtration on $\mathscr{M}^{\prime \prime}$, cf. (2.8.1). Then there exists a unique filtration $W$ on $(M, K)$ such that

$$
j^{*} W_{i}(M, K) \simeq W_{i}\left(M^{\prime}, K^{\prime}\right), \phi_{t, 1} W_{i}(M, K) \simeq L_{i} \phi_{t, 1}(M, K) .
$$

Put $\mathscr{M}=((M, F), K ; W)$. By Lemma 2.9 below, $F, V, W$ are compatible on $M$ and $\operatorname{Gr}_{i}^{W}(M, F)$ are quasi-unipotent and regular along $E_{0}$. We have

$$
\psi_{t} \mathscr{M} \simeq \psi_{t} \mathscr{M}^{\prime}, \quad \phi_{t, 1} \mathscr{M} \simeq \mathscr{M}^{\prime \prime} \quad \text { in } \operatorname{MHW}(E)^{(p)}
$$

by definition, where $W$ is the relative monodromy filtration with respect to $L$ (cf. 1.9 for $\left.\phi_{t, 1}\right)$. In particular $(2.2 .1)(2.3 .1)$ are satisfied, i.e. the specializations are well-defined. By 1.9 and $[19,(5.1 .4)]$, we have the canonical decomposition:

$$
\mathrm{Gr}_{k}^{W} \mathscr{M}=\mathscr{M}_{k, 1} \oplus \mathscr{M}_{k, 2} \quad \text { in } \operatorname{MF}_{h}\left(\mathscr{D}_{E}, \mathbf{Q}\right)
$$

such that supp $\mathscr{M}_{k, 2} \subset E_{0}$ and $\mathscr{M}_{k, 1}$ has no sub nor quotient with support in $E_{0}$. By [19, (3.2.2)], $\mathscr{M}_{k, 1}$ is the direct sum of $Z$-components of the extension of $\mathrm{Gr}_{k}^{W} \mathscr{M}^{\prime}$ to $E$ (cf. (2.7.5)) such that $Z \not \subset E_{0}$, because $j^{-1} \mathscr{M}_{k, 1} \simeq \mathrm{Gr}_{k}^{W} \mathscr{M}^{\prime}$ and can is strictly surjective by $[19,(5.1 .14)]$. On the other hand we have

$$
\mathscr{M}_{k, 2}=i_{*}\left(\operatorname{Ker~Gr}_{k}^{L} v: \mathrm{Gr}_{k}^{L} \mathscr{M}^{\prime \prime} \longrightarrow \mathrm{Gr}_{k-1}^{L} \psi_{t, 1} \mathscr{M}^{\prime}(-1)\right)
$$

by the identification (2.8.1), where $i: E_{0} \rightarrow E$. Therefore $\mathscr{M} \in \operatorname{MHW}(E)^{(p)}$. We see that this gives the inverse functor of (2.7.7), because it holds forgetting $F, W$, and $F, W$ (or $L=\phi_{t, 1} W$ ) on $\mathscr{M} \in \mathrm{MHW}(E)$ must satisfy (2.8.2)(2.8.4). If $X$ is singular, the above construction glues together globally (in fact, the Q-part is globally well-defind) and (2.8.5-6) are globally well-defined. Thus we get the assertion. To complete the proof of 2.8 , we have to show the following.

2.9. Lemma. Let $X$ be a complex manifold. Put $E=X \times \mathbf{C}$. Let $(M ; F, W)$ be a coherent filtered $\mathscr{D}_{E}$-Module with a finite increasing filtration $W . \quad$ If $(M, F)$ is quasi-unipotent and regular along $E_{0}=X \times\{0\}$ (cf. [19, (3.2.1)]) and $F, V, W$ on $V_{<0} M$ are compatible, they are compatible on $M$ and $\operatorname{Gr}_{i}^{W}(M, F)$ are quasi- 
unipotent and regular along $E_{0}$.

Proof. We first show the compatibility of $F, V, W$ on $V_{0} M$. By $[19,(1.2 .16 .(b))]$, it is enough to show the surjectivity of

$$
F_{p} W_{i} V_{0} M \longrightarrow F_{p} W_{i} \mathrm{Gr}_{0}^{V} M \quad \text { for any } p, i \text {. }
$$

Take $[u] \in F_{p} W_{i} \operatorname{Gr}_{0}^{V} M$ with $u \in F_{p} V_{0} M$. We have to show $u-u^{\prime} \in F_{p} W_{i} V_{0} M$ for some $u^{\prime} \in V_{<0} M$. By assumption $u t-u^{\prime \prime} \in F_{p} W_{i} V_{-1} M$ for some $u^{\prime \prime} \in F_{p} V_{<-1} M$. Take $u^{\prime} \in F_{p} V_{<0} M$ such that $u^{\prime} t=u^{\prime \prime}$, cf. [19, (3.2.1.2)]. Put $v=u-u^{\prime} \in F_{p} V_{0} M$. If $v \in W_{i} M$, the assertion is verified. If not, take $j>i$ such that $v \in W_{j} M, \operatorname{Gr}_{j}^{W} v$ $\neq 0$ in $\mathrm{Gr}_{j}^{W} M$. Then $\mathrm{Gr}_{j}^{W} v \in V_{<0} \mathrm{Gr}_{j}^{W} M$, because $v \in W_{j} V_{0} M$ and $\operatorname{Gr}_{j}^{W} \mathrm{Gr}_{0}^{V} v=0$ in $\mathrm{Gr}_{j}^{W} \mathrm{Gr}_{0}^{V} M$. Therefore $\mathrm{Gr}_{j}^{W} v t \neq 0$ in $\mathrm{Gr}_{j}^{W} M$ by $[19,(3.1 .4-5)]$. But this contradicts to $v t \in W_{i} M$. Thus the compatibility on $V_{0} M$ is proved.

We define the filtration $F^{\prime}$ on $W_{k} M, \mathrm{Gr}_{k}^{W} M$ by

$$
F_{p}^{\prime}\left(W_{k} M\right)=\sum\left(F_{p-i} V_{0} W_{k} M\right) \partial_{t}^{i}, F_{p}^{\prime}\left(\mathrm{Gr}_{k}^{W} M\right)=\sum\left(F_{p-i} V_{0} \mathrm{Gr}_{k}^{W} M\right) \partial_{t}^{i} .
$$

By the same argument as in $[19,(3.2 .2)]$, we have

$$
\begin{array}{ll}
F_{p}^{\prime} V_{\alpha}\left(W_{k} M\right)=F_{p} V_{\alpha} W_{k} M & \text { for } \alpha \leq 0 \\
\partial_{t}^{i}: F_{p} \mathrm{Gr}_{\alpha}^{V} W_{k} M \stackrel{\sim}{\longrightarrow} F_{p+i}^{\prime} \mathrm{Gr}_{\alpha+i}^{V}\left(W_{k} M\right) & \text { for }-1<\alpha \leq 0,
\end{array}
$$

(same for $\mathrm{Gr}_{k}^{W} M$ ). Combined with the compatibility on $V_{0} M$, we get the exact sequences:

$$
0 \longrightarrow\left(W_{k-1} M ; F^{\prime}, V\right) \longrightarrow\left(W_{k} M ; F^{\prime}, V\right) \longrightarrow\left(\mathrm{Gr}_{k}^{W} M ; F^{\prime}, V\right) \longrightarrow 0 .
$$

Therefore $F=F^{\prime}$ on $W_{k} M, \mathrm{Gr}_{k}^{W} M$ (because it holds on $W_{k} M$ for $k \gg 0$ ) and $F, V, W$ are compatible on $M$ (cf. $[19,(1.2 .14)]$ ). Now it remains to show

$$
\left(F_{p} V_{\alpha} \mathrm{Gr}_{k}^{W} M\right) t=F_{p} V_{\alpha-1} \mathrm{Gr}_{k}^{W} M \quad \text { for } \alpha<0 .
$$

But it is verified by decreasing induction on $k$, using

$$
0 \longrightarrow F_{p} V_{\alpha} W_{k-1} M \longrightarrow F_{p} V_{\alpha} W_{k} M \longrightarrow F_{p} V_{\alpha} \mathrm{Gr}_{k}^{W} M \longrightarrow 0 .
$$

This completes the proof of 2.9 and 2.8 .

2.10. Let $X$ be a separated reduced analytic space and $E$ a line bundle on $X$. Let $E_{0}$ be the zero section, $E^{*}$ its complement, and $i: E_{0} \rightarrow E, j: E^{*} \rightarrow E$ the natural inclusions. We define the full subcategory $\operatorname{MHW}\left(E^{*}\right)_{e x}$ (resp. $\left.\operatorname{MHW}(E)_{s p}^{(p)}\right)$ of $\operatorname{MHW}\left(E^{*}\right)\left(\right.$ resp. $\left.\operatorname{MHW}(E)^{(p)}\right)$ by the condition:

(2.10.1) for any open set $U$ of $X$ and a local trivialization $\left.E\right|_{U} \simeq U \times \mathbb{C}$, the restriction to $\left.E^{*}\right|_{U}$ (resp. $\left.\left.E\right|_{U}\right)$ belongs to $\mathrm{MHW}\left(U \times \mathbb{C}^{*}\right)_{e x}$ (resp. $\left.\operatorname{MHW}(U \times \mathbb{C})_{s p}\right)$,

cf. 2.7 for the notations. Here the condition (2.10.1)(i.e. $(2.7 .1-5)$ for the local 
representatives) is independent of the trivialization $\left.E\right|_{U} \simeq U \times \mathbf{C}$. Let $\operatorname{MHW}\left(E^{*}\right)_{e x}^{p}$ be the full subcategory of $\operatorname{MHW}\left(E^{*}\right)_{e x}$ such that $\operatorname{Gr}_{i}^{W}$ of the objects of $\mathrm{MHW}\left(E^{*}\right)_{e x}^{p}$ are extended to polarizable Hodge Modules on $E$, cf. (2.7.6). By 2.5, these full subcategories are abelian (i.e. stable by Ker, Coker), cf. 2.11 below or the proof of 2.5 for $\operatorname{MHW}\left(E^{*}\right)_{e x}$.

2.11. Proposition. With the notation of 2.10 , let $\mathscr{M}^{\prime} \in \mathrm{MHW}\left(E^{*}\right)_{e x}^{(p)}$. Then there exists functorially the extension $j_{*} \mathscr{M}^{\prime}$ (resp. $\left.j_{!} \mathscr{M}^{\prime}\right) \in \mathrm{MHW}(E)_{s p}^{(p)}$ of $\mathscr{M}^{\prime}$, unique up to a canonical isomorphism, such that its underlying perverse sheaf is isomorphic to $j_{*} K^{\prime}$ (resp. $\left.j_{!} K^{\prime}\right)$. For $\mathscr{M} \in \mathrm{MHW}(E)_{s p}^{(p)}$, we have $j^{-1} \mathscr{M} \in$ $\operatorname{MHW}(E)_{e x}^{(p)}$ and there is a unique and functorial morphism

$$
\mathscr{M} \longrightarrow j_{*} j^{-1} \mathscr{M} \quad\left(\text { resp. } j_{!} j^{-1} \mathscr{M} \longrightarrow \mathscr{M}\right)
$$

inducing the identity on $E^{*}$.

Proof. If $E$ is trivial, the assertion follows from 2.8. For example, take the inverse image of $\left(\mathscr{M}^{\prime}, \psi_{t, 1} \mathscr{M}^{\prime}(-1), N\right.$, id) to get $j_{*} \mathscr{M}^{\prime}$, and use the diagram

$$
\begin{array}{ll}
\psi_{t, 1} \mathscr{M} \stackrel{\text { id }}{\longrightarrow} & \psi_{t, 1} \mathscr{M} \\
\left.\operatorname{var} \uparrow||_{\text {can }} \quad \text { id } \uparrow\right\rfloor^{N} \\
\phi_{t, 1} \mathscr{M} \stackrel{\operatorname{Var}}{\longrightarrow} \psi_{t, 1} \mathscr{M}(-1)
\end{array}
$$

to get the morphism $\mathscr{M} \rightarrow j_{*} j^{*} \mathscr{M}$. In general, we have the assertion, except for the polarizability of $j_{*} \mathscr{M}^{\prime}, j_{!} \mathscr{M}^{\prime}$, by the uniqueness of $j_{*} \mathscr{M}^{\prime}, j_{!} \mathscr{M}^{\prime}$ and of the morphism (2.11.1). For the polarizability, it is enough to show the assertion for $j_{*} \mathscr{M}$ by 2.6. We first reduce to the case $\mathscr{M}^{\prime} \in \mathrm{MH}\left(E^{*}, n\right)$, i.e. $\mathrm{Gr}_{i}^{W} \mathscr{M}^{\prime}=0$ for $i \neq n$.

Put $\mathscr{M}=j_{*} \mathscr{M}^{\prime}$. We have the filtration $j_{*} W$ on $\mathscr{M}$ in $\operatorname{MHW}(E)_{s p}$, because $j_{*}$ is an exact functor. We have to show $j_{*} W$ splits canonically on $\operatorname{Gr}_{i}^{W} \mathscr{M}$. By the proof of 2.8, we have the global and canonical decomposition (2.8.5) in $\operatorname{MHW}(E)_{s p}$. Therefore it is enough to construct the splitting of $j_{*} W$ on $\mathscr{M}_{k, 2}$ in (2.8.5). Here note that any filtration of Hodge Modules is compatible with the decomposition by strict supports. Let $\mathrm{Sp}_{D}^{1} K, L\left(=\mathrm{Sp}_{D}^{1} W\right)$ and $W$ be as in 2.2-3, i.e. $W$ is the relative monodromy filtration of $\left(\mathrm{Sp}_{D}^{1} K, L\right)$. Then the Q-part $K_{k, 2}$ of $\mathscr{M}_{k, 2}$ is a direct factor of $\mathrm{Gr}_{k}^{L} \mathrm{Sp}_{D}^{1} K$, because $\mathrm{Sp}_{D}^{1}$ is exact and induces the identity on Perv $\left(\mathbf{Q}_{E_{0}}\right)$. We define the filtrations $\tilde{L}, \tilde{W}, \tilde{L}^{\prime}$ on $\operatorname{Sp}_{D}^{1} K \simeq j_{*} j^{*} \operatorname{Sp}_{D}^{1} K$ by

$$
\begin{aligned}
& \tilde{L}=j_{*} j^{*} L, \tilde{W}=j_{*} j^{*} W \\
& \tilde{L}_{k}^{\prime} \operatorname{Sp}_{D}^{1} K=N\left(\tilde{L}_{k} \operatorname{Sp}_{D}^{1} K\right)+\tilde{W}_{k-1} \tilde{L}_{k} \operatorname{Sp}_{D}^{1} K
\end{aligned}
$$


Then $\tilde{W}$ and $\tilde{W}[1]$ is the relative monodromy filtration of $\left(\operatorname{Sp}_{D}^{1} K, \tilde{L}\right)$ and $\left(\mathrm{Sp}_{D}^{1} K, \tilde{L}^{\prime}\right)$ respectively, cf. 1.10. If (locally) $E$ is trivial, i.e. $E \simeq X \times \mathbb{C}, \mathrm{Sp}_{D}^{1} K$ corresponds to

$$
\left(\psi_{t, 1} K \underset{\text { Var }}{\stackrel{\text { can }}{\rightleftarrows}} \phi_{t, 1} K\right)
$$

so that

$$
\begin{array}{lll}
W_{i}=\tilde{W}_{i}, & \tilde{L}_{k}^{\prime} \subset L_{k}=\tilde{L}_{k} & \text { on } \psi_{t, 1} K \\
\tilde{W}_{i-1}=W_{i}, & \tilde{L}_{k}^{\prime}=L_{k} \subset \tilde{L}_{k} & \text { on } \phi_{t, 1} K .
\end{array}
$$

Note that the functor $j_{*} j^{*}$ corresponds to the change of $\phi_{t, 1}$ so that Var becomes bijective, cf. (2.11.2). Therefore we get in general:

$$
\tilde{W}_{i-1} \subset W_{i} \subset \tilde{W}_{i}, \quad \tilde{L}_{k}^{\prime} \subset L_{k} \subset \tilde{L}_{k} .
$$

By 1.10 , we have a canonical splitting of $\tilde{L}$ on $\mathrm{Gr}_{k}^{\tilde{L^{\prime}}} \mathrm{Gr}_{i-1}^{W} \mathrm{Sp}_{D}^{1} K$. We shall see that this induces the desired splitting by the canonical morphism:

$$
\mathrm{Gr}_{k}^{\tilde{\tilde{I}}^{\prime}} \mathrm{Gr}_{k-1}^{\tilde{W}} \mathrm{Sp}_{D}^{1} K \longrightarrow \mathrm{Gr}_{k}^{L} \mathrm{Gr}_{k}^{W} \operatorname{Sp}_{D}^{1} K \longrightarrow K_{k, 2}
$$

where these morphisms are induced by (2.11.6) and by the canonical decomposition:

$$
\mathrm{Gr}_{k}^{L} \mathrm{Sp}_{D}^{1} K=\mathrm{Sp}_{D}^{1} K_{k, 1} \oplus K_{k, 2}
$$

Here $K_{k, a}$ is the underlying perverse sheaf of $\mathscr{M}_{k, a}(a=1,2)$, cf. (2.8.5), and $K_{k, 2}$ $=\mathrm{Gr}_{k}^{W} K_{k, 2}$ (because $N=0$ on $K_{k, 2}$ ). Now the assertion is local, and we may assume $E=X \times \mathbf{C}$ and use (2.11.4-5). Then the $\psi_{t, 1}$-part of $\widetilde{K}_{k, 2}$ is zero and the $\phi_{t, 1}$-part of the first morphism of (2.11.7) is the identity. Therefore we get the assertion by applying 1.10 to the $\phi_{t, 1}$-part, because the compatibility with the Hodge filtration follows from the uniqueness of the splitting, cf. the proof of (2.4.2).

Now we assume $\mathscr{M}^{\prime} \in \operatorname{MH}\left(E^{*}, n\right)$ and it is extended to a polarizable Hodge Module $\mathscr{M}$ on $E$, with no subobject supported in $E_{0}$. Locally we have $E \simeq X$ $\times \mathbb{C}$ and $\phi_{t, 1} j_{*} \mathscr{M}^{\prime}$ is identified with $\psi_{t, 1} \mathscr{M}(-1)=\psi_{t, 1} j_{*} \mathscr{M}^{\prime}(-1)$ by Var so that

$$
L_{i} \phi_{t, 1} j_{*} \mathscr{M}^{\prime}= \begin{cases}0 & \text { if } i<n \\ \operatorname{Im} N+\left(W_{i-2} \psi_{t, 1} \mathscr{M}\right)(-1) & \text { if } i \geq n\end{cases}
$$

by 1.9 , where $W$ on $\psi_{t, 1} \mathscr{M}$ is shifted by $n-1$, cf. [19, (5.1.6)]. Therefore we get locally: 


$$
\mathrm{Gr}_{i}^{W} j_{*} \mathscr{M}^{\prime} \simeq \begin{cases}0 & \text { if } i<n \\ \mathscr{M} & \text { if } i=n \\ i_{*} P \mathrm{Gr}_{i-2}^{W} \psi_{t, 1} \mathscr{M}(-1) & \text { if } i>n\end{cases}
$$

and it is enough to show the global polarizability of $\mathrm{Gr}_{i}^{W} j_{*} \mathscr{M}^{\prime}$ for $i>n$. By $[19,(5.1 .12)]$ we have the canonical decomposition:

$$
\mathrm{Gr}_{i}^{W} \operatorname{Sp}_{D}^{1} K \simeq \pi^{*} K_{i}^{\prime}[1] \oplus K_{i}^{\prime \prime}
$$

with $K_{i}^{\prime}, K_{i}^{\prime \prime} \in \operatorname{Perv}\left(\mathbf{Q}_{E_{0}}\right)$, where $\pi: E \rightarrow E_{0}$ is the projection and $W$ is the monodromy filtration shifted by $n$. Let $S: K \otimes K \rightarrow a_{E}^{!} \mathbf{Q}(-n)$ be a polarization of $M$. Then there is a pairing $S_{i}^{\prime}: K_{n+i}^{\prime} \otimes K_{n+i}^{\prime} \rightarrow a_{E_{0}}^{!} \mathbf{Q}(1-n)$ such that the restriction of $\mathrm{Gr}^{W}\left(\mathrm{Sp}_{D}^{1} S\right) \circ\left(\mathrm{id} \otimes N^{i}\right)$ to the first factor of $(2.11 .11)$ coincides with $\pi^{*} S_{i}^{\prime}$, where we have a change of sign as in [19, (5.2.2)]. If locally $E \simeq X$ $\times \mathbb{C}, S_{i}^{\prime}$ coincides with $\mathrm{Gr}^{W}\left({ }^{p} \psi_{t, 1} S\right) \circ\left(\mathrm{id} \otimes N^{i}\right)$ by the canonical isomorphism:

$$
K_{n+i}^{\prime} \simeq \mathrm{Gr}_{n-1+i}^{W} \psi_{t, 1} K \text {. }
$$

Therefore it is a polarization on the primitive part:

$$
P K_{n+i}^{\prime} \simeq P \mathrm{Gr}_{n-1+i}^{W} \psi_{t, 1} K \simeq\left(\mathrm{Gr}_{n+1+i}^{W} j_{*} K^{\prime}\right)(1),
$$

where the last isomorphism comes from (2.11.10). Thus it is enough to show the resulting isomorphism:

$$
P K_{i}^{\prime} \simeq\left(\mathrm{Gr}_{i+1}^{W} j_{*} K^{\prime}\right)(1)
$$

is globally well-defined for $i \geq n$. Consider an exact sequence:

$$
0 \longrightarrow \pi^{*} P K_{i}^{\prime}[1] \longrightarrow j_{*} j^{*} \pi^{*} P K_{i}^{\prime}[1] \stackrel{\rho}{\longrightarrow} P K_{i}^{\prime}(-1) \longrightarrow 0,
$$

where $\rho$ is characterized uniquely as the projection to the maximal quotient supported in $E_{0}$. On the other hand we have a morphism for $i \geq n$ :

$$
j_{*} j^{*} \pi^{*} P K_{i}^{\prime}[1] \longrightarrow \mathrm{Gr}_{i+1}^{W} j_{*} K
$$

induced by the composition:

$$
\begin{aligned}
& j_{*} j^{*} \pi^{*} K_{i}^{\prime}[1] \simeq j_{*} j^{*} \mathrm{Gr}_{i}^{W} \mathrm{Sp}_{D}^{1} j_{*} K^{\prime} \simeq \mathrm{Gr}_{i}^{\tilde{W}} \mathrm{Sp}_{\mathrm{D}}^{1} j_{*} K^{\prime} \\
& \longrightarrow \longrightarrow \mathrm{Gr}_{i+1}^{W} \mathrm{Sp}_{D}^{1} j_{*} K \longrightarrow \mathrm{Gr}_{i+1}^{L} \mathrm{Sp}_{D}^{1} j_{*} K \simeq \mathrm{Gr}_{i+1}^{W} j_{*} K
\end{aligned}
$$

where $W$ is the relative monodromy filtration of $\left(\operatorname{Sp}_{D}^{1} j_{*} K, L\right)$ and $\tilde{W}$ $=j_{*} j^{*} W$. Here we used (2.11.6) and $W_{i} \subset L_{i}$ for $i \geq n$, cf. (2.11.9). Then (2.11.13) is factored by $\rho$ (because supp $\mathrm{Gr}_{i+1}^{W} j_{*} K \subset E_{0}$ ) and we get a morphism:

$$
P K_{i}^{\prime}(-1) \longrightarrow \mathrm{Gr}_{i+1}^{W} j_{*} K \quad \text { for } i \geq n .
$$

If $E \simeq X \times \mathbb{C}$ (locally), $\rho$ corresponds to 


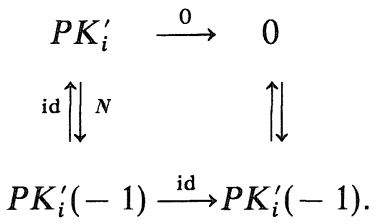

Therefore the morphism (2.11.14) coincides with (2.11.12) by definition, cf. (2.11.9-10). This completes the proof of 2.11 .

2.12. Lemma. With the notation of 2.10 , let

$$
0 \longrightarrow \mathscr{M}^{\prime} \longrightarrow \mathscr{M} \longrightarrow \mathscr{M}^{\prime \prime} \longrightarrow 0
$$

be an exact sequence in $\mathrm{MHW}\left(E^{*}\right)$ such that $\mathscr{M} \in \mathrm{MHW}\left(E^{*}\right)_{\text {ex }}$ and each $\mathrm{Gr}_{i}^{W}$ of (2.12.1) has a splitting (e.g. $\left.\mathscr{M} \in \mathrm{MHW}\left(E^{*}\right)_{\text {ex }}^{p}\right)$. Then $\mathscr{M}^{\prime}, \mathscr{M}^{\prime \prime} \in \mathrm{MHW}\left(E^{*}\right)_{\text {ex }}$ and we have a canonical (and functorial) exact sequence:

$$
0 \longrightarrow j_{*} \mathscr{M}^{\prime} \longrightarrow j_{*} \mathscr{M} \longrightarrow j_{*} \mathscr{M}^{\prime \prime} \longrightarrow 0
$$

$$
\text { (resp. } 0 \longrightarrow j_{!} \mathscr{M}^{\prime} \longrightarrow j_{!} \mathscr{M} \longrightarrow j_{!} \mathscr{M}^{\prime \prime} \longrightarrow 0 \text { ). }
$$

Proof. By the functoriality of $j_{*}, j_{!},(2.12 .2)$ is clear if $\mathscr{M}^{\prime}, \mathscr{M}^{\prime \prime} \in M H W\left(E^{*}\right)_{e x}$. Therefore the assertion is local and we may assume $X$ smooth and $E=X \times \mathbb{C}$. By 2.5 it is enough to verify (2.7.1-5) only for $\mathscr{M}^{\prime \prime}$. By the splitting of $\mathrm{Gr}_{i}^{W}$ (2.12.1) and the extension of $\mathrm{Gr}_{i}^{W} \mathscr{M}$, we have an extension of $\mathrm{Gr}_{i}^{W}$ (2.12.1) with its splitting (cf. [19, (3.2.2), (5.1.7)]). Then we get (2.7.1) and (2.7.5). Let $\tilde{M}$, $\tilde{M}^{\prime \prime}$ be the underlying $\mathscr{D}_{E}$-Modules of $j_{*} \mathscr{M}, j_{*} \mathscr{M}^{\prime \prime}$ (i.e. $\tilde{M}^{\prime \prime}$ is regular holonomic and $\left.\operatorname{DR}\left(\tilde{M}^{\prime \prime}\right) \simeq j_{*} K^{\prime \prime} \otimes \mathbb{C}\right)$. Then the above splitting implies the surjectivity of

$$
F_{p} V_{\alpha} \mathrm{Gr}_{i}^{W} \tilde{M} \longrightarrow F_{p} V_{\alpha} \mathrm{Gr}_{i}^{W} \tilde{M}^{\prime \prime} \quad \text { for } \alpha<0
$$

where $F, W$ on $\tilde{M}^{\prime \prime}$ are the quotient filtrations, and we use $[19,(3.1 .5)]$ for the filtration $V$. In fact the image of this morphism in $V_{\alpha} \mathrm{Gr}_{i}^{W} \tilde{M}^{\prime \prime}$ is

$$
V_{\alpha} \mathrm{Gr}_{i}^{W} \tilde{M}^{\prime \prime} \cap j_{*} F_{p} \mathrm{Gr}_{i}^{W} M^{\prime \prime}
$$

by the above splitting (because $\alpha<0$ ) and this contains clearly $F_{p} V_{\alpha} \mathrm{Gr}_{i}^{W}$. We verify by induction on $i$ the surjectivity of $(*)(* *)$ in the diagram:



for $\alpha<0$. Therefore $F, V, W$ are compatible on $V_{<0} \tilde{M}^{\prime \prime}$, and for $(2.7 .2-3)$ it is enough to show 


$$
F_{p} V_{<0} \tilde{M}^{\prime \prime}=V_{<0} \tilde{M}^{\prime \prime} \cap j_{*} F_{p} M^{\prime \prime}
$$

or equivalently $\left(F_{p} V_{\alpha} \tilde{M}^{\prime \prime}\right) t=F_{p} V_{\alpha-1} \tilde{M}^{\prime \prime}$ for $\alpha<0$ by $[19,(3.2 .2)]$, but this follows from the surjectivity of $(* *)$ for $i \gg 0$. Finally (2.7.4) follows from 1.2, using the above splitting, cf. the proof of 2.5 .

\section{(2.c) Direct Images}

2.13. Let $X$ be a separated reduced complex analytic space, and $U_{I}, V_{I}, \mathrm{pr}_{I J}$ as in 2.1. By definition $[19,(2.1 .20)]$, an object $(M, F)$ of $\operatorname{MF}\left(\mathscr{D}_{X}\right)$ is a family of filtered $\mathscr{D}_{V_{I}}$-Modules $\left\{\left(M_{I}, F\right)\right\}$ with isomorphisms :

$$
u_{I J}:\left(\operatorname{pr}_{I J}\right)_{*}\left(M_{J}, F\right) \simeq\left(M_{I}, F\right) \quad \text { on } V_{I} \backslash\left(U_{I} \backslash U_{J}\right)
$$

for $I \subset J$, such that $\mathrm{Gr}^{F} M_{I}$ are annihilated by the Ideal of $U_{I}$ (in particular, $\left.\operatorname{supp} M_{I} \subset U_{I}\right)$ and $\left\{u_{I J}\right\}$ satisfy the compatibility conditions:

$$
u_{I K}=u_{I J} \circ\left(\operatorname{pr}_{I J}\right)_{*} u_{J K} \quad \text { on } V_{I} \backslash\left(U_{I} \backslash U_{K}\right)
$$

for $I \subset J \subset K . \quad \operatorname{Here} \operatorname{MF}\left(\mathscr{D}_{X}\right)$ is independent of the choice of $U_{i}, V_{i}$ by the same argument as in 2.1. In particular, for $(M, F) \in \mathrm{MF}\left(\mathscr{D}_{X}\right)$ and for any open subset $U$ of $X$ with a closed embedding $U \rightarrow V$ such that $V$ smooth, there exists uniquely a filtered $\mathscr{D}_{V}$-Module, which represents $(M, F)$ on $V \supset U$ (called the local representative of $(M, F)$ associated to $U \rightarrow V)$. Then we can define $C F\left(\mathscr{D}_{X}\right), K F\left(\mathscr{D}_{X}\right)$ and $D F\left(\mathscr{D}_{X}\right)$ as usual, because for $i: X \rightarrow Y$ a closed immersion of complex manifolds, $\left(M^{*}, F\right)$ is filtered acyclic iff so is $i_{*}\left(M^{*}, F\right)$.

Let $f: X \rightarrow Y$ be a proper morphism of separated reduced complex analytic spaces. Let $X=\cup_{i} U_{i}, Y=\cup_{i} U_{i}^{\prime}$ be locally finite open coverings with closed immersions: $U_{i} \rightarrow V_{i}, U_{i}^{\prime} \rightarrow V_{i}^{\prime}$ such that $V_{i}, V_{i}^{\prime}$ smooth, $f\left(U_{i}\right) \subset U_{i}^{\prime}$ and $f: U_{i}$ $\rightarrow U_{i}^{\prime}$ is extended to $f_{i}: V_{i} \rightarrow V_{i}^{\prime}$. Let $(M, F)=\left\{\left(M_{I}, F\right)\right\} \in \operatorname{MF}\left(\mathscr{D}_{X}\right)$ where $\left(M_{I}, F\right) \in \operatorname{MF}\left(\mathscr{D}_{V_{I}}\right)$. Assume $\operatorname{Gr}_{p}^{F} M_{I}$ are flabby. We define

$$
\left(f_{I}\right)_{!}\left(M_{I}, F\right):=\mathrm{DR}^{-1} \circ\left(f_{I}\right)_{!} \circ \tilde{\mathrm{DR}}\left(M_{I}, F\right) \in C F\left(\mathscr{D}_{V_{I}^{\prime}}\right)
$$

(cf. $[19, \S 2.2]$ for the definition of $\mathrm{DR}^{-1}, \tilde{\mathrm{DR}}$ ), where $f_{I}=\Pi_{i \in I} f_{i}$ and $\left(f_{I}\right)$ ! in the right hand side is the topological direct image with proper supports. Then $\left(f_{I}\right)_{1}\left(M_{I}, F\right)$ belongs to $C F\left(\mathscr{D}_{U_{I}^{\prime}}\right)$ and is independent of the choice of $f_{i}$, because $\left(i_{f_{I}}\right)_{*}\left(M_{I}, F\right)$ is independent of $f_{I}$ by $[19,(3.2 .6)]$. Taking its zero extension to $Y$, we get

$$
f_{!}\left(M_{I}, F\right) \in C F\left(\mathscr{D}_{Y}\right)
$$

Then $u_{I J}$ induces a morphism in $C F\left(\mathscr{D}_{Y}\right)$ :

$$
f_{!}\left(u_{I J}\right): f_{!}\left(M_{J}, F\right) \longrightarrow f_{!}\left(M_{I}, F\right)
$$

because $\tilde{\mathrm{DR}}\left(M_{I}, F\right)$ is isomorphic to a quotient complex of $\left(\operatorname{pr}_{I J}\right)_{!} \tilde{\mathrm{DR}}\left(M_{J}, F\right)$ by 
$u_{I J}$. We define

$$
f_{*}(M, F) \in C F\left(\mathscr{D}_{Y}\right)
$$

to be the single complex associated to the double complex of Č ech whose $(p, q)$ component is $\bigoplus_{|I|-1=-p}\left(f_{!}\left(M_{I}, F\right)\right)^{q}$.

If $\mathrm{Gr}_{p}^{F} M_{I}$ are not flabby, we take Godement's canonical flabby resolution and truncate by $\tau_{\leq 2 d(I)+1}$, where $d(I)=\min \left\{\operatorname{dim} V_{i}: i \in I\right\}$. Then we get a triple complex and $f_{*}(M, F)$ is defined to be the associated single complex. Because this definition is functorial, we get the direct image:

$$
f_{*}: D F\left(\mathscr{D}_{X}\right) \longrightarrow D F\left(\mathscr{D}_{Y}\right) \text {. }
$$

Let $f: X \rightarrow Y$ be as above. We define $\operatorname{MF}_{h}\left(\mathscr{D}_{X}, \mathbb{Q}\right)\left(\operatorname{resp} . \mathrm{MF}_{h} \mathrm{~W}\left(\mathscr{D}_{X}, \mathbb{Q}\right)\right)$ by the same way as above, where they are defined in $[19,(5.1 .1)]$ (resp. $[19,(5.1 .14)]$ ) in the smooth case. By [3], an object of $\mathrm{MF}_{h}\left(\mathscr{D}_{X}, \mathbb{Q}\right)$ is a pair of $(M, F)=\left\{\left(M_{I}, F\right)\right\} \in \mathrm{MF}_{h}\left(\mathscr{D}_{X}\right)$ and $K \in \operatorname{Perv}\left(\mathbb{Q}_{X}\right)$ with isomorphisms: $\left.\mathrm{DR}\left(M_{I}\right) \simeq \mathbb{C} \otimes K\right|_{U_{I}}$ compatible with $u_{I J}$. We say that $f_{*}(M, F)$ is strict, if its any local representative is strict. If $f_{*}(M, F)$ is strict and if $\mathscr{H}^{j} f_{*}(M, F) \in$ $\mathrm{MF}_{h}\left(\mathscr{D}_{Y}\right)$, we define:

$$
\mathscr{H}^{j} f_{*}(M, F, K)=\left(\mathscr{H}^{j} f_{*}(M, F),{ }^{p} \mathscr{H}^{j} f_{*} K\right) \in \mathrm{MF}_{h}\left(\mathscr{D}_{\mathrm{Y}}, \mathbb{Q}\right),
$$

where $\operatorname{DR}\left(\mathscr{H}^{j} f_{*} M\right) \simeq \mathbb{C} \otimes{ }^{p} \mathscr{H}^{j} f_{*} K$ is induced by the direct image of the isomorphism:

$$
\bigoplus_{|I|-1=-} \cdot\left(j_{I}\right)_{!} \tilde{\mathrm{DR}}\left(M_{I}\right) \simeq \mathbb{C} \otimes K
$$

where $j_{I}: U_{I} \rightarrow X$, cf. [3].

2.14. Theorem. Let $f: X \rightarrow Y$ be a projective morphism of separated reduced complex analytic spaces, and $\mathscr{M}=((M, F), K ; W) \in \operatorname{MHW}(X)^{p}$. Then $f_{*}(M, F)$ is strict and $\mathscr{H}^{j} f_{*} \mathscr{M}:=\left(\mathscr{H}^{j} f_{*}(M, F),{ }^{p} \mathscr{H}^{j} f_{*} K ; W[j]\right)$ belongs to $\mathrm{MHW}(Y)^{p}$, where $W$ is the induced filtration by $f_{*}$, i.e. $W_{i} \mathscr{H}^{j} f_{*}(M, K)=\operatorname{Im}\left(\mathscr{H}^{j} f_{*} W_{i}(M, K)\right.$ $\left.\rightarrow \mathscr{H}^{j} f_{*}(M, K)\right)$. For a holomorphic function $g$ on $Y$, put $h=g f$. If the vanishing cycle functors along $h$ are well-defined for $\mathscr{M}$ (cf. 2.3), they are welldefined along $g$ for $\mathscr{H}^{j} f_{*} \mathscr{M}$ and we have the canonical isomorphisms in $\operatorname{MHW}(Y)^{p}$ :

$$
\psi_{g} \mathscr{H}^{j} f_{*} \mathscr{M} \simeq \mathscr{H}^{j} f_{*} \psi_{h} \mathscr{M}, \phi_{g, 1} \mathscr{H}^{j} f_{*} \mathscr{M} \simeq \mathscr{H}^{j} f_{*} \phi_{h, 1} \mathscr{M} .
$$

Proof. Let $U_{i}, V_{i}, U_{i}^{\prime}, V_{i}^{\prime}$ and $f_{i}$ be as in 2.13. We may assume $U_{i}=f^{-1}\left(U_{i}^{\prime}\right)$ and $f_{i}: V_{i} \rightarrow V_{i}^{\prime}$ is projective (because $f$ is projective). Therefore, if $\mathscr{M}$ is pure of weight $n$ (i.e. $\operatorname{Gr}_{i}^{W} \mathscr{M}=0$ for $\left.i \neq n\right), f_{*}(M, F)$ is strict, and $\mathscr{H}^{j} f_{*} \mathscr{M} \in \operatorname{MH}(Y, n$ $+j)^{p}$ by $[19,(5.3 .1)]$. Moreover the vanishing cycle functors along $h$ are welldefined for $\mathscr{M}$ by definition so that $\psi_{h} \mathscr{M}, \phi_{h, 1} \mathscr{M} \in \mathrm{MHW}(X)^{p}$, and the weight filtration of $\mathscr{H}^{j} f_{*} \psi_{h} \mathscr{M}$ (resp. $\left.\mathscr{H}^{j} f_{*} \phi_{h, 1} \mathscr{M}\right) \in \mathrm{MHW}(Y)$ is the monodromy 
filtration shifted by $n-1+j$ (resp. $n+j$ ) and is given by the induced filtration $\mathscr{H}^{j} f_{*} W[j]$ by $[19,(5.3 .4)]$. Therefore the assertion follows from the next two propositions and $[19,(3.4 .13)]$ (because the filtration $\mathscr{H}^{j} f_{*} L$ on $\mathscr{H}^{j} f_{*} \psi_{h} \mathscr{M}, \mathscr{H}^{j} f_{*} \phi_{h, 1} \mathscr{M} \quad$ coincides $\quad$ with $\quad \psi_{g} \mathscr{H}^{j} f_{*} W[-1], \phi_{g, 1} \mathscr{H}^{j} f_{*} W \quad$ on $\left.\psi_{g} \mathscr{H}^{j} f_{*} \mathscr{M}, \phi_{g, 1} \mathscr{H}^{j} f_{*} \mathscr{M}\right)$.

2.15. Proposition. Let $f: X \rightarrow Y$ be a proper morphism of separated reduced complex analytic spaces, and $\mathscr{M}=((M, F), K ; W) \in \mathrm{MF}_{h} \mathrm{~W}\left(\mathscr{D}_{X}, \mathbb{Q}\right), c f .2 .13$. Assume $f_{*} \mathrm{Gr}_{i}^{W}(M, F)$ is strict, $\mathscr{H}^{j} f_{*} \mathrm{Gr}_{i}^{W}(M, F) \in \mathrm{MF}_{h}\left(\mathscr{D}_{Y}\right)$ and $\mathscr{H}^{j} f_{*} \mathrm{Gr}_{i}^{W} \mathscr{M} \in$ $\mathrm{MH}(Y, i+j)^{(p)}$ for any $i, j$. Then $f_{*}(M, F)$ is strict, $\mathscr{H}^{j} f_{*}(M, F) \in \mathrm{MF}_{h}\left(\mathscr{D}_{Y}\right)$ and we have the weight spectral sequence in $\mathrm{MF}_{h}\left(\mathscr{D}_{Y}, \mathbf{Q}\right)$ :

$$
E_{1}^{-i, i+j}=\mathscr{H}^{j} f_{*} \operatorname{Gr}_{i}^{W}((M, F), K) \Longrightarrow \mathscr{H}^{j} f_{*}((M, F), K),
$$

degenerating at $E_{2}$, such that $d_{1}$ is a morphism of Hodge Modules (hence strict) and

$$
E_{2}^{-i, i+j} \simeq \mathrm{Gr}_{i}^{W} \mathscr{H}^{j} f_{*}((M, F), K) \in \mathrm{MH}(Y, j+i)^{(p)},
$$

i.e. $\left(\mathscr{H}^{j} f_{*}(M, F, K), \mathscr{H}^{j} f_{*} W[j]\right) \in \mathrm{MHW}(Y)^{(p)}$, where $W$ is the induced filtration $\mathscr{H}^{j} f_{*} W$ on $\mathscr{H}^{j} f_{*}(M, K)$ as in 2.14. Moreover $\left(f_{*}(M, F)\right.$, Dec $\left.f_{*} W\right)$ is strict and

$$
\mathscr{H}^{j}\left(f_{*}(M, F), \operatorname{Dec} f_{*} W\right) \simeq\left(\mathscr{H}^{j} f_{*}(M, F), \mathscr{H}^{j} f_{*} W[j]\right) .
$$

Proof. The Q-part of (2.15.1-2) and $\mathscr{H}^{j} f_{*} \mathrm{Gr}_{i}^{W}((M, F), K), \mathscr{H}^{j} f_{*}((M, F), K)$ are globally well-defined, if $f_{*}(M, F)$ is strict and $\mathscr{H}^{j} f_{*}(M, F) \in \mathrm{MF}_{h}\left(\mathscr{D}_{Y}\right)$. Therefore the assertion is local and we may assume $Y$ smooth, because the assertion on the polarizability is trivial (cf. $[19,(5.2 .13)]$ ). Then we have the weight spectral sequence $(2.15 .1)$ in $\mathrm{MG}_{h}(\mathscr{B}, \mathbf{Q})$ by $[19,(5.2 .17-21)]$ (cf. $[19,(5.1 .1)]$ for the definition of $\mathrm{MG}_{h}(\mathscr{B}, \mathbf{Q})$ ). By assumption $E_{1}^{-i, i+j} \in$ $\operatorname{MH}(Y, i+j)^{(p)}$ and $d_{r}$ are morphisms in $\mathrm{MG}_{h}(\mathscr{B}, \mathbf{Q})$. Therefore $d_{1}$ is a morphism in $\mathrm{MH}(Y, i+j)^{(p)}$ so that $E_{2}^{-i, i+j} \in \mathrm{MH}(Y, i+j)^{(p)}$ and $d_{r}=0$ for $r \geq 2$ by $[19,(5.1 .14),(5.1 .11)]$. Then the isomorphism $(2.15 .2)$ in $\mathrm{MG}_{h}(\mathscr{B}, \mathbf{Q})$ implies $\mathscr{H}^{j} f_{*}(M, F, K) \in \mathrm{MF}_{h}\left(\mathscr{D}_{X}, \mathbf{Q}\right)$ (i.e. $f_{*}(M, F)$ is strict) and the filtration $W$ of $\mathscr{H}^{j} f_{*}(M, F, K)$ in $\mathrm{MG}_{h}(\mathscr{B}, \mathbf{Q})$ associated to $(2.15 .1)$ is actually a filtration in $\mathrm{MF}_{h}\left(\mathscr{D}_{Y}, \mathbb{Q}\right)$, which gives the weight filtration of $\mathscr{H}^{j} f_{*}(M, F, K)$ and coincides with $\mathscr{H}^{j} f_{*} W$ on $\mathscr{H}^{j} f_{*}(M, K)$. The last assertion follows from [19, (1.3.7)], because $f_{*} \mathrm{Gr}_{i}^{W}(M, F) \simeq \mathrm{Gr}_{i}^{W} f_{*}(M, F)$ is strict and $\operatorname{Dec} f_{*} W$ is well-defined on $f_{*}(M, F)$.

2.16. Proposition. Let $f: X \rightarrow Y$ be a proper morphism of complex manifolds. Put $X=\tilde{X} \times \mathbf{C}, \tilde{Y}=Y \times \mathbb{C}, \tilde{f}=f \times$ id $: \tilde{X} \rightarrow \tilde{Y} \quad$ and $\quad \mathscr{M}=((M, F), K ; W) \in$ $\mathrm{MF}_{h} \mathrm{~W}(\mathscr{D} \tilde{X}, \mathbb{Q})$. Assume:

(2.16.1) $(M, F)$ is quasi-unipotent and regular along $X \times\{0\}$,

(2.16.2) the three filtrations $F, V, W$ on $M$ are compatible, 
(2.16.3) the relative monodromy filtration $W$ on $\left(\psi_{t}(M, F, K), L\right)$ and $\left(\phi_{t, 1}(M, F, K), L\right)$ exists, cf. 1.1,

(2.16.4) for $\left(\left(M_{k}, F\right), K_{k} ; W\right)=\mathrm{Gr}_{k}^{L}\left(\psi_{t}(M, F, K), W\right), \mathrm{Gr}_{k}^{L}\left(\phi_{t, 1}(M, F, K), W\right)$, $f_{*} \operatorname{Gr}_{i}^{W}\left(M_{k}, F\right)$ is strict, $\mathscr{H}^{j} f_{*} \mathrm{Gr}_{i}^{W}\left(M_{k}, F, K_{k}\right) \in \mathrm{MH}(Y, i+j)^{(p)}$ and the induced filtration $\mathscr{H}^{j} f_{*} W$ on $\mathscr{H}^{j} f_{*}\left(M_{k}, K_{k}\right)$ is the monodromy filtration shifted by $k$ for any $i, j, k$.

Then we have:

(2.16.5) $\tilde{f}_{*} \mathrm{Gr}_{i}^{W}(M, F), \tilde{f}_{*}(M, F)$ are strict on a neighborhood of $Y \times\{0\}$,

(2.16.6) $\quad \mathscr{H}^{j} \tilde{f}_{*} \operatorname{Gr}_{i}^{W}(M, F), \mathscr{H}^{j} \tilde{f}_{*}(M, F)$ are quasi-unipotent and regular along $Y \times\{0\}$,

(2.16.7) $\quad F, V, \mathscr{H}^{j} \tilde{f}_{*} W$ on $\mathscr{H}^{j} \tilde{f}_{*} M$ are compatible.

Moreover, for $\left(\left(M^{\prime}, F\right), K^{\prime} ; L, W\right)=\left(\psi_{t}(M, F, K) ; L, W\right),\left(\phi_{t, 1}(M, F, K) ; L, W\right)$, we have:

(2.16.8) $\operatorname{Dec}\left(f_{*} W\right)$ on $f_{*}\left(M^{\prime} ; F, L\right)$ is well-defined $[19,(1.3 .7)]$ and $\left(\operatorname{Gr}_{k}^{L} f_{*}\left(M^{\prime}, F\right), \operatorname{Dec}\left(f_{*} W\right)\right),\left(f_{*}\left(M^{\prime}, F\right), \operatorname{Dec}\left(f_{*} W\right)\right)$ are strict,

(2.16.9) we have the limit weight spectral sequence in $\mathrm{MHW}(Y)^{(p)}$ :

$$
\begin{aligned}
E_{1}^{-k, k+j}= & \left(\mathscr{H}^{j} f_{*} \operatorname{Gr}_{k}^{L}\left(M^{\prime}, F, K^{\prime}\right), \mathscr{H}^{j} f_{*} W[j]\right) \\
& \Longrightarrow\left(\mathscr{H}^{j} f_{*}\left(M^{\prime}, F, K^{\prime}\right), \mathscr{H}^{j} f_{*} W[j]\right)
\end{aligned}
$$

degenerating at $E_{2}\left(i t\right.$ is canonically isomorphic to $\psi_{t}\left(\right.$ or $\left.\phi_{t, 1}\right)$ of (2.15.1) applied to $\mathscr{M}$ and $\tilde{f}$, forgetting $W$ ),

(2.16.10) $\mathscr{H}^{j} f_{*} W[j]$ is the relative monodromy filtration of $\left(\mathscr{H}^{j} f_{*}\left(M^{\prime}, F, K^{\prime}\right)\right.$, $\left.\mathscr{H}^{j} f_{*} L[j]\right)$,

(2.16.11) $\quad F, \mathscr{H}^{j} f_{*} W, \mathscr{H}^{j} f_{*} L$ on $\mathscr{H}^{j} f_{*} M^{\prime}$ are compatible.

Proof. By definition (cf. 2.13), $f_{*}\left(\left(M^{\prime}, F\right) ; L, W\right)$ is represented by a complex such that $F, L, W$ are compatible on each component, and $f_{*}$ commutes with $\mathrm{Gr}_{i}^{W}, \mathrm{Gr}_{k}^{L}$. Then by (2.16.4), $\operatorname{Gr}_{k}^{L} \mathrm{Gr}_{i}^{W} f_{*}\left(M^{\prime}, F\right)$ is strict, and the filtration $f_{*} L$ on $\mathrm{Gr}_{i}^{W} f_{*}\left(M^{\prime}, F\right)$ has the canonical splitting by 1.5 applied to $\left(\left(M^{\prime}, F\right) ; W, L\right)$. Here $\mathrm{Gr}_{i}^{f * W}$ is abbreviated by $\mathrm{Gr}_{i}^{W}$ (same for $L$ ). Then by $[19,(1.3 .8)]$, $\operatorname{Dec}\left(f_{*} W\right)$ on $f_{*}\left(\left(M^{\prime}, F\right), L\right)$ is well-defined so that Dec commutes with $\mathrm{Gr}_{k}^{L}$. Moreover $\left(f_{*} \mathrm{Gr}_{k}^{L}\left(M^{\prime}, F\right), \quad \operatorname{Dec}\left(f_{*} W\right)\right)$ are strict, $\left(\mathscr{H}^{j} f_{*} \mathrm{Gr}_{k}^{L}\left(\left(M^{\prime}, F\right), K^{\prime}\right)\right.$, $\left.\mathscr{H}^{j} f_{*} W[j]\right) \in \mathrm{MHW}(Y)^{(p)}$ and its weight filtration $\mathscr{H}^{j} f_{*} W[j]$ is the monodromy filtration shifted by $j+k$ by (2.16.4) and 2.15. Consider a spectral sequence:

$$
\begin{aligned}
E_{1}^{-k, k+j}=\mathscr{H}^{j}\left(f_{*} \operatorname{Gr}_{k}^{L}\left(M^{\prime}, F\right)\right. & \left., \operatorname{Dec}\left(f_{*} W\right)\right) \\
& \Longrightarrow \mathscr{H}^{j}\left(f_{*}\left(M^{\prime}, F\right), \operatorname{Dec}\left(f_{*} W\right)\right)
\end{aligned}
$$


in $\operatorname{MGW}(\mathscr{B})$ the category of inductive systems of $\operatorname{MG}(\mathscr{B})$, cf. $[19,(2.1 .1)(5.1 .1)]$. It is compatible with the Q-part of (2.16.9) forgetting $W$, and $E_{1}^{-k, k+j}$ underlies $\left(\mathscr{H}^{j} f_{*} \mathrm{Gr}_{k}^{L}\left(\left(M^{\prime}, F\right), K^{\prime}\right), \quad \mathscr{H}^{j} f_{*} W[j]\right) \in \mathrm{MHW}(Y)^{(p)}$. Because $d_{1}$ of (2.16.12) preserves the filtration $\operatorname{Dec}\left(f_{*} W\right)$, it underlies a morphism in $\operatorname{MHW}(Y)^{(p)}$ so that $E_{2}^{-k, k+j}$ of (2.16.12) underlies an object of $\operatorname{MHW}(Y)^{(p)}$, whose weight filtration is the monodromy filtration shifted by $j+k$ (because it holds for $E_{1}^{-k, k+j}$ by the above argument.) We show $d_{r}=0$ for $r \geq 2$ by induction on $r$. Assume $E_{2}^{-k, k+j} \simeq E_{r}^{-k, k+j}$ for $r \geq 2$. Because $d_{r}$ preserves $\operatorname{Dec}\left(f_{*} W\right)$, it underlies a morphism in $\operatorname{MHW}(Y)^{(p)}$, hence it is enough to show $\mathrm{Gr}^{W} d_{r}=0$; but this follows from the following argument (cf. [24]):

Let $u: M^{1} \rightarrow M^{2}$ be a morphism of graded modules with the action of $N$ such that $N\left(M_{i}^{a}\right) \subset M_{i-2}^{a}$, then $u=0$, if $N^{i}: M_{p_{a}+i}^{a} \rightarrow M_{p_{a}-i}^{a}$ with $p_{1}>p_{2}$. (For the proof, restrict $u$ to the primitive part of $M^{1}$.) Therefore we get an isomorphism in $\mathrm{MG} \tilde{\mathrm{W}}(\mathscr{B})$ :

$$
E_{2}^{-k, k+j} \simeq \operatorname{Gr}_{k}^{L} \mathscr{H}^{j}\left(f_{*}\left(M^{\prime}, F\right), \operatorname{Dec}\left(f_{*} W\right)\right),
$$

which implies $\mathscr{H}^{j}\left(f_{*}\left(M^{\prime}, F\right), \operatorname{Dec}\left(f_{*} W\right)\right) \in \mathrm{MF}_{h} \mathrm{~W}\left(\mathscr{D}_{Y}\right)$ (i.e. $\left(f_{*}\left(M^{\prime}, F\right), \operatorname{Dec}\left(f_{*} W\right)\right)$ is strict), and the filtration $L$ of $\mathscr{H}^{\mathrm{j}}\left(f_{*}\left(M^{\prime}, F\right), \operatorname{Dec}\left(f_{*} W\right)\right)$ in $\operatorname{MG\tilde {W}}(\mathscr{B})$ associated to the spectral sequence $(2.16 .12)$ is actually a filtration in $\mathrm{MF}_{h} \mathrm{~W}\left(\mathscr{D}_{Y}\right)$, which coincides with $\mathscr{H}^{j} f_{*} L$ on $\mathscr{H}^{j} f_{*} M^{\prime}$. In particular, we get (2.16.8) and (2.16.11) (cf. $[19,(1.3 .3)]$, because $f_{*} W[j]$ and $\operatorname{Dec}\left(f_{*} W\right)$ induce the same filtration on $\mathscr{H}^{j} f_{*} M^{\prime}$. Moreover we get $(2.16 .10)$ by $(2.16 .13)$ (and by the above argument). Because (2.16.12-13) are compatible with $\mathbf{Q}$-structure (forgetting $W$ ), we get

$$
\mathrm{Gr}_{k}^{L}\left(\mathscr{H}^{j}\left(f_{*}\left(\left(M^{\prime}, F\right), K^{\prime}\right), \mathscr{H}^{j} f_{*} W[j]\right) \in \operatorname{MHW}(Y)^{(p)}\right.
$$

and

$$
\left(\mathscr{H}^{j}\left(f_{*}\left(\left(M^{\prime}, F\right), K^{\prime}\right), \mathscr{H}^{j} f_{*} W[j]\right) \in \operatorname{MHW}(Y)^{(p)}\right.
$$

by the canonical splitting of $L$ on $\mathrm{Gr}^{W}$ in 1.5. Therefore we get (2.16.9).

By $[19,(3.3 .17)],(2.16 .5-6)$ follows from (2.16.8). Moreover $\tilde{f}_{*} \mathrm{Gr}^{W}(M ; F, V)$ and $\tilde{f}_{*}(M ; F, V)$ are strict on a neighborhood of $Y \times\{0\}$ so that the filtration $V$ on $\mathscr{H}^{\mathrm{j}} \tilde{f}_{*} \mathrm{Gr}_{i}^{W} M$ and $\mathscr{H}^{\mathrm{j}} \tilde{f}_{*} M$ coincides with $\mathscr{H}^{j} f_{*} V$. Therefore we get (2.16.7), using the spectral sequence:

$$
E_{1}^{-i, i+j}=\mathscr{H}^{j} \tilde{f}_{*} \mathrm{Gr}_{i}^{W}(M ; F, V) \Longrightarrow \mathscr{H}^{j} \tilde{f}_{*}(M ; F, V)
$$

In fact, (2.16.14) degenerates at $E_{2}$ (because $\mathrm{Gr}^{V}$ of (2.16.14) is isomorphic to (2.16.12) forgetting $\operatorname{Dec}\left(f_{*} W\right)$ ) and $d_{1}$ of (2.16.14) is strict on $Y \times\{0\}$ by $[19,(3.3 .3-5)]$. Hence $F, V, W$ are compatible on $\mathscr{H}^{j} \tilde{f}_{*} M$ by the same argument as above, because $V$ is trivial on $Y \times \mathbf{C}^{*}$. 


\section{(2.d) Mixed Hodge Modules}

2.17. Let $f: X \rightarrow \bar{X}$ be an open immersion of separated reduced complex analytic spaces, such that locally $\bar{X} \backslash X=g^{-1}(0)_{\text {red }}$ for a locally defind holomorphic function $g$. We say the direct image $j_{*}$ (resp. $j_{!}$) is well-defined for $\mathscr{M} \in \mathrm{MHW}(X)$, if there exists an extension $\tilde{\mathscr{M}} \in \mathrm{MHW}(\bar{X})$ of $\mathscr{M}$, denoted by $j_{*} \mathscr{M}$ (resp. $\left.j_{!} \mathscr{M}\right)$, such that the following conditions are satisfied:

(2.17.1) for any holomorphic function on an open set $U$ of $\bar{X}$ such that $g^{-1}(0)_{\text {red }}=U \backslash X$, the vanishing cycle functors along $g$ are welldefined for $\left.\tilde{M}\right|_{U}$,

(2.17.2) $\quad \tilde{K}=j_{*} K$ (resp. $j_{!} K$ ), where $\tilde{K}, K$ are the underlying perverse sheaves.

By $2.11, j_{*} \mathscr{M}$ (resp. $j_{!} \mathscr{M}$ ) is unique (if it exists).

We define a full subcategory $\operatorname{MHM}(X)$ of $\operatorname{MHW}(X)$, called the category of mixed Hodge Modules, as follows:

For $\mathscr{M} \in \mathrm{MHW}(X), \mathscr{M}$ belongs to $\operatorname{MHM}(X)$, iff, for any complex manifold $Y$, and open subset $U$ of $X \times Y$, and any (finite) number of holomorphic functions $g_{1}, g_{2}, \ldots$, the following condition is satisfied inductively for $r \geq 1$ :

the vanishing cycle functors along $g_{r}$ are well-defined for $\mathscr{M}_{r}$, and the direct images $\left(j_{r}\right)_{*},\left(j_{r}\right)_{\text {! }}$ are well-defined for $j_{r}^{-1} \mathscr{M}_{r}$,

where $\quad j_{r}: U \backslash g_{r}^{-1}(0) \rightarrow U, \mathscr{M}_{1}=\left.\left(M \otimes \mathbf{Q}_{Y}^{H}\left[d_{Y}\right]\right)\right|_{U} \quad$ and $\quad \mathscr{M}_{r}=\psi_{g_{r-1}} \mathscr{M}_{r-1}$, $\phi_{g_{r-1}, 1} \mathscr{M}_{r-1},\left(j_{r-1}\right)_{*} j_{r-1}^{-1} \mathscr{M}_{r-1}$ and $\left(j_{r-1}\right)_{i} j_{r-1}^{-1} \mathscr{M}_{r-1}$ for $r>1$.

Here $\mathbb{Q}_{Y}^{H}\left[d_{Y}\right]=\left(\left(\Omega_{Y}^{d_{Y}}, F\right), \mathbb{Q}_{Y}\left[d_{Y}\right] ; W\right)$ with $\operatorname{Gr}_{-i}^{F}=\mathrm{Gr}_{i}^{W}=0$ for $i \neq d_{Y}$ $(=\operatorname{dim} Y)$, and for complex manifolds $X_{1}, X_{2}$ and $\mathscr{M}_{i}=\left(\left(\mathscr{M}_{i}, F\right), K_{i} ; W\right) \in$ $M F_{h}\left(\mathscr{D}_{X_{2}}, \mathbb{Q}\right)$, we define $M_{1} \otimes M_{2}=((M, F), K ; W)$ by

$$
\begin{aligned}
& M=M_{1} \bigotimes M_{2}\left(=\left(\mathrm{pr}_{1}^{-1} M_{1} \otimes \mathrm{pr}_{2}^{-1} M_{2}\right) \underset{\mathrm{pr}_{1}^{-1} \mathcal{O}_{X_{1}} \otimes \mathrm{pr}_{2}^{-1} \mathcal{O}_{X_{2}}}{\otimes} \mathcal{O}_{X_{1} \times X_{2}}\right) \\
& K=K_{1} \otimes K_{2} \\
& F_{p} M=\sum_{i+j=p}\left(F_{i} M_{1} \bigotimes F_{j} M_{2}\right) \\
& W_{k}(M, K)=\sum_{i+j=k}\left(W_{i}\left(M_{1}, K_{1}\right) \bigotimes W_{j}\left(M_{2}, K_{2}\right)\right)
\end{aligned}
$$

(The case $X_{i}$ singular is reduced to this case, because this definition is compatible with closed immersions.) Then the condition for $\operatorname{MHM}(X)$ is local, and for a closed immersion $i: X \rightarrow Y$, we have an equivalence of categories:

$$
i_{*}: \operatorname{MHM}(X) \stackrel{\sim}{\longrightarrow} \operatorname{MHM}_{X}(Y),
$$

where $\operatorname{MHM}_{X}(Y)$ is the full subcategory of $\operatorname{MHM}(Y)$ consisting of the objects supported in $X$. (In fact the condition (2.17.3) is invariant by closed immersions.) 
By definition, $\operatorname{MHM}(X)$ is stable by Tate twist $(n)$, smooth pull-backs, vanishing cycle functors and $j_{*} j^{-1}, j_{!} j^{-1}$ for open immersions $j: U \rightarrow X$ such that $X \backslash U$ is a locally principal divisor. (In fact, these functors are compatible with $\otimes \mathbf{Q}_{Y}^{H}\left[d_{Y}\right]$.) Here, for $f: X \rightarrow Y$ a smooth morphism of complex manifolds with $\operatorname{dim} X-\operatorname{dim} Y=l$ and $\mathscr{M}=((M, F), K ; W) \in \mathrm{MF}_{h}\left(\mathscr{D}_{Y}, \mathbf{Q}\right)$, we define $\mathscr{H}^{-l} f^{!} \mathscr{M}=((\tilde{M}, F), \tilde{K} ; W)$ by

$$
\begin{aligned}
& (\tilde{M} ; F, W)=\omega_{X} \otimes_{f^{-1} \mathscr{O}_{Y}} f^{-1}\left((M ; F, W[-l]) \otimes \omega_{Y}^{-1}\right) \\
& (\tilde{K}, W)=f^{!}(K, W[-l])[-l]
\end{aligned}
$$

and put $\mathscr{H}^{l} f^{*} \mathscr{M}=\left(\mathscr{H}^{-l} f^{!} \mathscr{M}\right)(-l)$. (The singular case is reduced to this case by definition.) Then we have a natural isomorphism:

$$
\mathscr{H}^{l} f^{*} \mathscr{M} \simeq \mathscr{M} \otimes \mathbf{Q}_{Z}^{H}\left[d_{z}\right],
$$

if $X=Y \times Z$ and $f=\operatorname{pr}_{1}$ is smooth. For $\mathscr{M}=((M, F), K ; W) \in \mathrm{MF}_{h}\left(\mathscr{D}_{X}, \mathbb{Q}\right)$, we define $\mathscr{M}(n)=((\tilde{M}, F), \tilde{K} ; W)$ by

$$
\begin{aligned}
& (\tilde{M}, \tilde{K})=(M, K)(n), W_{i}(\tilde{M}, \tilde{K})=\left(W_{i+2 n}(M, K)\right)(n) \\
& F_{p} \tilde{M}=\left(F_{p-n} M\right)(n),
\end{aligned}
$$

where $(n)=\bigotimes_{\mathrm{z}}(2 \pi i)^{n} \mathbb{Z}$, cf. $[19,(2.0 .2)] . \quad$ By 2.6, $\operatorname{MHM}(X)$ is stable by the dual functor $\mathbf{D}$, because $\mathbf{D}$ commutes with $\otimes \mathbf{Q}_{Y}^{H}\left[d_{Y}\right]$ (up to Tate twist) and vanishing cycle functors, and $j_{*} j^{-1}, j_{!} j^{-1}$ are exchanged by $\mathbf{D}$.

We define the category of polarizable mixed Hodge Modules by

$$
\operatorname{MHM}(X)^{p}=\operatorname{MHM}(X) \cap \operatorname{MHW}(X)^{p} .
$$

Then it is stable by Tate twist $(n)$, dual functor $\mathbf{D}$, vanishing cycle functors and $j_{*} j^{-1}, j_{!} j^{-1}$ for $j$ as above. By 2.14 , it is also stable by $\mathscr{H}^{j} f_{*}$ for a projective morphism $f$, because $\mathscr{H}^{j} f_{*}$ commutes with $\bigotimes \mathbf{Q}_{Z}^{H}\left[d_{Z}\right]$.

2.18. Let $j: X \rightarrow \bar{X}$ be an open immersion of separated reduced complex analytic spaces such that $\bar{X} \backslash X$ is an analytic subset. We say that $j$ is admissible to $\mathscr{M} \in \operatorname{MHM}(X)$, if $\mathscr{M}$ is extendable to $\bar{X}$, i.e. there exists $\tilde{\mathscr{M}} \in \operatorname{MHW}(\bar{X})$ such that $j^{-1} \tilde{\mathscr{M}} \simeq \mathscr{M}$.

Let $f: X \rightarrow Y$ be a morphism of separated reduced complex analytic spaces. We say that $f$ is projectively compactifiable, if there is a factorization $f$ $=\bar{f} \circ j$ such that $\bar{f}$ is a composition of projective morphisms and $j$ is an open immersion whose complement $\bar{X} \backslash X$ is a locally principal divisor. Two projective compactifications $f=\bar{f}_{i}^{\circ} j_{i}(i=1,2)$ are called (projectively) equivalent, if there is a projective compactification $f=\bar{f} \circ j$ with a commutative diagram: 




such that $\pi_{1}, \pi_{2}$ are compositions of projective morphisms. We say a projective compactification $f=\bar{f} \circ j$ is admissible to $\mathscr{M} \in \operatorname{MHM}(X)$, if so is $j$. If $M \in \operatorname{MHM}(X)^{p}$, this condition depends only on the equivalent class of projective compactifications by 2.14 and 2.19 below. Here we use 2.11 and 3.21 to assure $j_{*} \mathscr{M}, j_{!} \mathscr{M} \in \operatorname{MHM}(\bar{X})^{p}$. In this case, we define

$$
\begin{gathered}
\mathscr{H}^{j} f_{*} \mathscr{M}=\mathscr{H}^{j} \bar{f}_{*}\left(j_{*} \mathscr{M}\right) \\
\mathscr{H}^{j} f_{!} \mathscr{M}=\mathscr{H}^{j} \bar{f}_{*}\left(j_{!} \mathscr{M}\right)
\end{gathered}
$$

which depend only on the equivalence class of projective compactification of $f$. (Note that a composition of projective morphisms is projective locally on the image of the morphism. For the polarizability we can use Deligne's uniqueness of the decomposition.)

2.19 Proposition. Let $f: X \rightarrow Y$ be a morphism of separated reduced complex analytic spaces. Then we have cohomological functors $(j \in \mathbb{Z})$ :

$$
\mathscr{H}^{j} f^{*}, \mathscr{H}^{j} f^{!}: \operatorname{MHM}(Y) \longrightarrow \operatorname{MHM}(X)
$$

compatible with

$$
{ }^{p} \mathscr{H}^{j} f^{*},{ }^{p} \mathscr{H}^{j} f^{!}: \operatorname{Perv}\left(\mathbf{Q}_{Y}\right) \longrightarrow \operatorname{Perv}\left(\mathbf{Q}_{X}\right)
$$

Proof. Assume first $f$ is a closed immersion such that $X=\cap g_{i}^{-1}(0)_{\text {red }}$ for holomorphic functions $g_{1}, \ldots, g_{r}$ on $Y$. Put $U_{i}=\left\{g_{i} \neq 0\right\}$ and let

$$
j_{I}: U_{I}=\bigcap_{i \in I} U_{i} \longrightarrow Y
$$

be natural inclusions. We define $\mathscr{H}^{j} f^{*} \mathscr{M}$ (resp. $\mathscr{H}^{j} f^{!} \mathscr{M}$ ) by the $j^{\text {th }}$ cohomology of the complex in $\operatorname{MHM}(Y)$ :

$$
\left.\bigoplus_{|I|=-.}\left(j_{I}\right)_{!} j_{I}^{-1} \mathscr{M} \quad \text { (resp. } \underset{|I|=.}{\bigoplus}\left(j_{I}\right)_{*} j_{I}^{-1} \mathscr{M}\right)
$$

whose component of degree 0 is $\left(j_{\phi}\right)_{1} j_{\phi}^{-1} \mathscr{M}:=\mathscr{M}$. (Here we use the equivalence (2.17.5).) We can easily check that this definition is compatible with ${ }^{p} \mathscr{H}^{j} f^{*} K,{ }^{p} \mathscr{H}^{j} f^{!} K$ on the underlying perverse sheaves by $[3,(3.1 .14)]$ (here $K$ is 
represented by a complex of flabby sheaves), and independent of the choice of $\left\{g_{i}\right\}$ (i.e. we have a natural isomorphism between the underlying perverse sheaf of $\mathscr{H}^{j} f^{*} \mathscr{M}$ and ${ }^{p} \mathscr{H}^{j} f^{*} K$, and the composition of these isomorphisms for different $\left\{g_{i}\right\}$ induces an isomorphism in $\operatorname{MHM}(X)$.) Therefore we get the assertion in the closed immersion case, because ${ }^{p} \mathscr{H}^{j} f^{*} K,{ }^{p} \mathscr{H}^{j} f^{!} K$ are globally well-defined.

We now assume $f$ has a factorization $f=p \circ i$ such that $p$ is smooth and $i$ is a closed immersion. Then we define

$$
\mathscr{H}^{j} f^{*} \mathscr{M}=\mathscr{H}^{j-l} i^{*} \mathscr{H}^{l} p^{*} \mathscr{M} \quad\left(\text { resp. } \mathscr{H}^{j} f^{!} \mathscr{M}=\mathscr{H}^{i+l} i^{*} \mathscr{H}^{-l} p^{!} \mathscr{M}\right),
$$

where $l$ is the relative dimension of $p$. By the same argument as above, it is enough to show the independence of the factorization (in the above sense), because any morphism has such a factorization locally on $X$. Let $f=p_{j} \circ i_{j}(j$ $=1,2$ ) be two factorization. We may assume the existence of a smooth morphism $p$ with a commutative diagram:

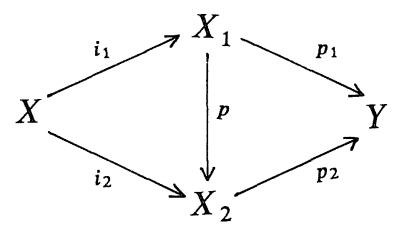

using the fiber product $X_{1} \times{ }_{Y} X_{2}$. Therefore the assertion is reduced to the case $f$ is a closed immersion (by replacing $f$ with $i_{2}$ ) and we have to show the canonical isomorphism:

$$
\mathscr{H}^{j} f^{*} \mathscr{M} \simeq \mathscr{H}^{j-l} i^{*} \mathscr{H}^{l} p^{*} \mathscr{M}
$$

for a factorization $f=p \circ i$ as above. (We show the assertion only for $\mathscr{H}^{j} f^{*}$, because the argument for $\mathscr{H}^{j} f^{!}$is similar.) Because the assertion is local, we may assume $l=1$ (by factorizing $p$ ) and $X=\left\{g_{1}=\cdots=g_{d}=0\right\}$ (in $Y$ ), $X_{1}=Y$ $\times \Delta$ so that $p=\operatorname{pr}_{1}$ and $\operatorname{Im} i=\operatorname{Im} f \times\{0\}$, where $\Delta$ is an open $\operatorname{disc}$ and $g_{1}, \ldots, g_{d}$ are holomorphic functions on $Y$. By definition, the both sides of (2.19.1) are given by the cohomology of the complexes:

$$
\begin{aligned}
& \bigoplus_{|I|=-.}\left(j_{I}\right)_{!} j_{I}^{-1} \mathscr{M} \\
& \bigoplus_{|I|=-.}\left(\left(j_{I}\right)_{!} j_{I}^{-1} \mathscr{M}\right) \otimes C\left(j_{!} \mathbf{Q}_{\Delta^{*}}^{H}[1] \longrightarrow \mathbf{Q}_{\Delta}^{H}[1]\right)
\end{aligned}
$$

where $j_{I}: U_{I} \rightarrow Y, U_{I}=\left\{\Pi_{i \in I} g_{i}=0\right\}, j: \Delta^{*} \rightarrow \Delta$ and $\Delta^{*}=\Delta \backslash\{0\}$. Therefore the isomorphism (2.19.1) follows from the canonical exact sequence:

$$
0 \longrightarrow i_{*} \mathbf{Q}_{\{0\}}^{H} \longrightarrow j_{!} \mathbf{Q}_{\Delta^{*}}^{H}[1] \longrightarrow \mathbf{Q}_{\Delta}^{H}[1] \longrightarrow 0,
$$

where $i:\{0\} \rightarrow \Delta$. 
2.20. Corollary (of the proof of 2.19). Let $i: X \rightarrow Y$ be a closed immersion of separated reduced complex analytic spaces. Put $j: U=Y \backslash X \rightarrow Y$. Then we have natural functors for $k \in \mathbb{Z}$ :

$$
\mathscr{H}^{k} j_{!} j^{-1}, \mathscr{H}^{k} j_{*} j^{-1}: \operatorname{MHM}(Y) \longrightarrow \operatorname{MHM}(Y)
$$

compatible with

$$
{ }^{p} \mathscr{H}^{k} j_{!} j^{-1},{ }^{p} \mathscr{H}^{k} j_{*} j^{-1}: \operatorname{Perv}\left(\mathbf{Q}_{Y}\right) \longrightarrow \operatorname{Perv}\left(\mathbf{Q}_{Y}\right)
$$

so that we have functorial long exact sequences:

$$
\begin{aligned}
& \longrightarrow \mathscr{H}^{k} j_{!} j^{-1} \mathscr{M} \longrightarrow \mathscr{H}^{k} \mathscr{M} \longrightarrow i_{*} \mathscr{H}^{k} i^{*} \mathscr{M} \longrightarrow \mathscr{H}^{k+1} j_{!} j^{-1} \mathscr{M} \longrightarrow \\
& \longrightarrow i_{*} \mathscr{H}^{k} i^{!} \mathscr{M} \longrightarrow \mathscr{H}^{k} \mathscr{M} \longrightarrow \mathscr{H}^{k} j_{*} j^{-1} \mathscr{M} \longrightarrow i_{*} \mathscr{H}^{k+1} i^{!} \mathscr{M} \longrightarrow
\end{aligned}
$$

compatible with the corresponding exact sequences in $\operatorname{Perv}\left(\mathbb{Q}_{Y}\right)$, where $\mathscr{H}^{k} \mathscr{M}$ $=\mathscr{M}($ if $k=0)$ and 0 (otherwise). If $X$ is a locally principal divisor, $\mathscr{H}^{k} j_{!} j^{-1}$ $=\mathscr{H}^{k} j_{*} j^{-1}=0$ for $k \neq 0$ and $\mathscr{H}^{0} j_{!} j^{-1}, \mathscr{H}^{0} j_{*} j^{-1}$ coincide with $j_{!} j^{-1}, j_{*} j^{-1}$ in 2.17.

Proof. The assertion is local, because the underlying exact sequences of (2.20.1) in $\operatorname{Perv}\left(\mathbf{Q}_{Y}\right)$ are globally well-defined. Therefore we may assume $X=\left\{g_{1}=\cdots\right.$ $\left.=g_{d}=0\right\}$, and let $j_{I}: U_{I} \rightarrow Y$ be as in the proof of 2.18. Then we define $\mathscr{H}^{k} j_{!} j^{-1} \mathscr{M}$ (resp. $\mathscr{H}^{k} j_{*} j^{-1} \mathscr{M}$ ) by the $k^{\text {th }}$ cohomology of the complexes:

$$
\underset{|I|-1=-.}{\oplus}\left(j_{I}\right)_{!} j_{I}^{-1} \mathscr{M}\left(\text { resp. } \underset{|I|-1=.}{\oplus}\left(j_{I}\right)_{*} j_{I}^{-1} \mathscr{M}\right),
$$

where the component of degree $k$ is assumed to be zero for $k>0$ (resp. $k<0$ ), i.e. $I=\phi$. We verify the well-definedness (as in the proof of 2.18). Then the exact sequence (2.20.1) and the last assertion are clear.

Remark. With the notation of $2.19, j$ has only one equivalence class of projective compactification, and $\mathscr{H}^{k} j_{!} j^{-1}, \mathscr{H}^{k} j_{*} j^{-1}$ in 2.19 are compatible with the definition in (2.18.1) in the polarizable case (by the last assertion of 2.20 and the independence of $\left\{g_{i}\right\}$ on the blow up of $Y$ ).

In the proof of the next proposition, we need essentially 3.21.

2.21. Proposition. Let $f: X \rightarrow Y$ be as in 2.19. Assume $f$ has a factorization $f$ $=p \circ i$ such that $p$ is smooth and $i$ is a closed immersion. Then the functors $\mathscr{H}^{j} f^{*}, \mathscr{H}^{j} f^{!}$in 2.18 preserve the polarizability.

Proof. By 3.21, the polarizability is preserved by smooth pullbacks. Therefore we may assume $f$ is a closed immersion, and let $j: U \rightarrow Y$ be as in 2.20. Then by the exact sequences (2.20.1), it is enough to show the polarizability of $\mathscr{H}^{k} j_{!} j^{-1} \mathscr{M}, \mathscr{H}^{k} j_{*} j^{-1} \mathscr{M}$, because $\mathscr{H}^{k} j_{!} j^{-1} \mathscr{M}=\mathscr{H}^{-k} j_{*} j^{-1} \mathscr{M}$ 
$=0$ for $k>0$ and $\mathscr{H}^{k} \mathscr{M}=0$ for $k \neq 0$. Let $\pi: \tilde{Y} \rightarrow Y$ be the blow-up of $X$, and $\tilde{j}: U \rightarrow \tilde{Y}$ the natural inclusion. Then

$$
\mathscr{H}^{k} j_{!} j^{-1} \mathscr{M}=\mathscr{H}^{k} \pi_{*}\left(\tilde{j}_{!} j^{-1} \mathscr{M}\right), \mathscr{H}^{k} j_{*} j^{-1} \mathscr{M}=\mathscr{H}^{k} \pi_{*}\left(\tilde{j}_{*} j^{-1} \mathscr{M}\right)
$$

by the above remark, if $\tilde{j}_{!} j^{-1} \mathscr{M}, \quad \tilde{j}_{*} j^{-1} \mathscr{M} \in \operatorname{MHM}(\tilde{Y})^{p}$. But $\tilde{j}_{!} j^{-1} \mathscr{M}$, $\tilde{j}_{*} j^{-1} \mathscr{M} \in \operatorname{MHM}(\tilde{Y})$ follows from 2.19 (and by the stability of $\tilde{j}_{!} \tilde{j}^{-1}, \tilde{j}_{*} j^{-1}$ ) and the polarizability from 2.11 and 3.21. Then the assertion follows from 2.14 .

\section{(2.e) Beilinson's Functor and its Applications}

2.22. Proposition. Let $i: X \rightarrow Y$ be a closed immersion of separated reduced analytic spaces such that $X=g^{-1}(0)_{\text {red }}$ with a holomorphic function $g$ defined on $Y$, and $i_{g}: Y \rightarrow Y \times S$ the immersion by graph, where $S=C$. Let $j: U:=Y X \rightarrow Y$ and $j_{g}:\{g \neq t\} \rightarrow Y \times S$ be the natural inclusions of their complements, where $t$ is the coordinate of $S=C$. We define the functor:

$$
\xi_{g}: \operatorname{MHM}(Y)^{(p)} \longrightarrow \operatorname{MHM}(Y)^{(p)}
$$

by

$$
\xi_{g} \mathscr{M}=\psi_{t, 1}\left(j_{g}\right)_{!}\left(j_{g}\right)^{-1}\left(M \otimes \mathbf{Q}_{S}^{H}[1]\right) .
$$

Then we have canonical and functorial exact sequences in $\operatorname{MHM}(Y)^{(p)}$ :

$$
\begin{gathered}
0 \longrightarrow \psi_{g, 1} \mathscr{M} \longrightarrow \xi_{g} \mathscr{M} \longrightarrow \mathscr{M} \longrightarrow 0 \\
0 \longrightarrow j_{1} j^{-1} \mathscr{M} \longrightarrow \xi_{g} \mathscr{M} \longrightarrow \phi_{g, 1} \mathscr{M} \longrightarrow 0 .
\end{gathered}
$$

Proof. We have an exact sequence in $\operatorname{MHM}(Y)^{(p)}$ :

$$
0 \longrightarrow\left(i_{g}\right)_{*} \mathscr{M} \longrightarrow\left(j_{g}\right)_{i} j_{g}^{-1} \tilde{\mathscr{M}} \longrightarrow \tilde{\mathscr{M}} \longrightarrow 0
$$

where $\tilde{\mathscr{M}}=\mathscr{M} \otimes \mathbf{Q}_{S}^{H}[1]$. Taking $\psi_{t, 1}$, we get (2.22.1). Let $i_{0}: Y \times\{0\} \rightarrow Y$ $\times S, j_{0}: Y \times S^{*} \rightarrow Y \times S$ and $\tilde{j}:\{g \neq t, g \neq 0\} \rightarrow Y \times S$ be the natural inclusions, where $S^{*}=S \backslash\{0\}$. Then we have an exact sequence:

$$
0 \longrightarrow\left(i_{0}\right)_{*} j_{!} j^{-1} \mathscr{M} \longrightarrow \tilde{j}_{!} \tilde{j}^{-1} \tilde{\mathscr{M}} \longrightarrow\left(j_{g}\right)_{!} j_{g}^{-1} \tilde{\mathscr{M}} \longrightarrow 0
$$

by applying $\left(j_{g}\right)_{!} j_{g}^{-1}$ to

$$
0 \longrightarrow\left(i_{0}\right)_{*} \mathscr{M} \longrightarrow\left(j_{0}\right)_{1} j_{0}^{-1} \tilde{\mathscr{M}} \longrightarrow \tilde{\mathscr{M}} \longrightarrow 0 .
$$

Taking $\phi_{t, 1}$ of (2.22.3-4), we get an exact sequence

$$
0 \longrightarrow j_{!} j^{-1} \mathscr{M} \longrightarrow \phi_{t, 1} \tilde{j}_{!} \tilde{j}^{-1} \tilde{\mathscr{M}} \longrightarrow \phi_{g, 1} \mathscr{M} \longrightarrow 0,
$$

because $\phi_{t, 1} \tilde{\mathscr{M}}=0$ and $\phi_{t, 1}\left(i_{0}\right)_{*} \simeq$ id. We get

$$
\psi_{t, 1} \tilde{j}_{!} \tilde{j}^{-1} \tilde{\mathscr{M}} \simeq \xi_{g} \mathscr{M}
$$


by applying $\psi_{t, 1}$ to $(2.22 .4)$. Then the assertion follows from the canonical isomorphism:

$$
\text { can }: \psi_{t, 1} \tilde{j}_{!} \tilde{j}^{-1} \tilde{\mathscr{M}} \stackrel{\sim}{\longrightarrow} \phi_{t, 1} \tilde{j}_{!} \tilde{j}^{-1} \tilde{\mathscr{M}} \text {. }
$$

Remark. The above proof of (2.22.2) was pointed out to me by Kashiwara. He also remarked the exact sequences:

$$
\begin{gathered}
0 \longrightarrow \xi_{g} \mathscr{M} \longrightarrow \mathscr{M} \oplus \xi_{g} j_{*} j^{-1} \mathscr{M} \longrightarrow j_{*} j^{-1} \mathscr{M} \longrightarrow 0 \\
0 \longrightarrow \xi_{g} \mathscr{M} \longrightarrow \phi_{g, 1} \mathscr{M} \oplus \xi_{g} j_{*} j^{-1} \mathscr{M} \longrightarrow \psi_{g, 1} \mathscr{M}(-1) \longrightarrow 0
\end{gathered}
$$



By (2.22.5), Beilinson's functor $\Xi_{g}$ used in [1] should correspond to $\xi_{g} j_{*}$.

2.23. Corollary. For $\mathscr{M}^{\bullet} \in D_{X}^{b} \operatorname{MHM}(Y)^{(p)}$, we have a canonical (and functorial) isomorphism in $D_{X}^{b} \operatorname{MHM}(Y)^{(p)}$ :

$$
\mathscr{M}^{\bullet} \simeq \phi_{g, 1} \mathscr{M}^{\circ}
$$

so that we have an equivalence of categories:

$$
D^{b} \operatorname{MHM}_{X}(Y)^{(p)} \stackrel{\sim}{\longrightarrow} D_{X}^{b} \operatorname{MHM}(Y)^{(p)}
$$

where $\phi_{g, 1}$ gives a quasi-inverse. Here $D^{b}$ means the derived category of bounded complexes of an abelian category and $D_{X}^{b}$ is its full subcategory of the objects whose cohomological supports are contained in $X\left(c f .(2.17 .5)\right.$ for $\left.\operatorname{MHM}_{X}(Y)\right)$.

Proof. Because $\xi_{g}$ and $\phi_{g, 1}$ are exact and induces the identity on $\operatorname{MHM}_{X}(Y)^{(p)}$, we have the quasi-isomorphisms:

$$
\mathscr{M}^{\bullet} \longleftarrow \xi_{g} \mathscr{M}^{\bullet} \longrightarrow \phi_{g, 1} \mathscr{M}^{\bullet}
$$

and we get (2.23.1), then (2.23.2) is clear.

Remark. The isomorphism: $\phi_{g, 1} \mathscr{M}^{\circ} \simeq \phi_{g, 1} \mathscr{M}^{\circ}$ obtained by applying $\phi_{g, 1}$ to (2.23.1) is the identity by the above proof (because we may assume $M^{\bullet} \in D^{b} \operatorname{MHM}_{X}(Y)^{(p)}$.)

2.24. Corollary. For $\mathscr{M} \in \operatorname{MHM}(Y)^{(p)}$, we have canonical isomorphisms:

$$
\phi_{g, 1} j_{!} j^{-1} \mathscr{M} \simeq \psi_{g, 1} \mathscr{M}, \phi_{g, 1} j_{*} j^{-1} \mathscr{M} \simeq \psi_{g, 1} \mathscr{M}(-1)
$$

so that $\phi_{g, 1}$ of the canonical morphisms:

$$
j_{!} j^{-1} \mathscr{M} \longrightarrow \mathscr{M}, \mathscr{M} \longrightarrow j_{*} j^{-1} \mathscr{M}
$$

are identified with can, Var. Therefore we have canonical isomorphisms in $D_{X}^{b} \operatorname{MHM}(Y)^{(p)}$ :

$$
\begin{aligned}
& C\left(j_{!} j^{-1} \mathscr{M} \longrightarrow \mathscr{M}\right) \simeq C\left(\text { can }: \psi_{g, 1} \mathscr{M} \longrightarrow \phi_{g, 1} \mathscr{M}\right) \\
& C\left(\mathscr{M} \longrightarrow j_{*} j^{-1} \mathscr{M}\right) \simeq C\left(\operatorname{Var}: \phi_{g, 1} \mathscr{M} \longrightarrow \psi_{g, 1} \mathscr{M}(-1)\right)
\end{aligned}
$$


so that $i_{*} i^{*} \mathscr{M}$ and $i_{*} i^{!} \mathscr{M}[1]$ are represented by these complexes, i.e. we have the canonical exact sequences:

$$
\begin{aligned}
& 0 \longrightarrow i_{*} \mathscr{H}^{-1} i^{*} \mathscr{M} \longrightarrow \psi_{g, 1} \mathscr{M} \stackrel{\text { can }}{\longrightarrow} \phi_{g, 1} \mathscr{M} \longrightarrow i_{*} \mathscr{H}^{0} i^{*} \mathscr{M} \longrightarrow 0 \\
& 0 \longrightarrow i_{*} \mathscr{H}^{0} i^{!} \mathscr{M} \longrightarrow \phi_{g, 1} \mathscr{M} \stackrel{\text { var }}{\longrightarrow} \psi_{g, 1} \mathscr{M}(-1) \longrightarrow i_{*} \mathscr{H}^{1} i^{!} \mathscr{M} \longrightarrow 0
\end{aligned}
$$

Proof. This follows from the proof of 2.19 and the commutative diagrams:

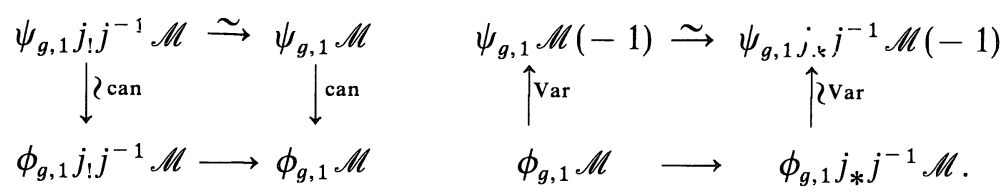

Remark. If $i: X \rightarrow Y$ is a closed immersion such that $X=\left\{g_{1}=\cdots=g_{d}\right.$ $=0\}, \mathscr{H}^{j} f^{*} \mathscr{M}\left(\right.$ resp. $\left.\mathscr{H}^{j} f^{!} \mathscr{M}\right)$ is isomorphic to the cohomology of the single complex associated to the $n$-ple complex obtained by iterating the functors

$$
\left.C\left(\text { can }: \psi_{g_{l}, 1} \longrightarrow \phi_{g_{l}, 1}\right) \text { (resp. } C\left(\operatorname{Var}: \phi_{g_{l}, 1} \longrightarrow \psi_{g_{l}, 1}(-1)\right)[-1]\right) \text {. }
$$

2.25. Lemma. Let $f: X \rightarrow Y$ be a morphism of complex manifolds. Put $d$ $=\operatorname{dim} X-\operatorname{dim} Y$. Assume $f$ is non-characteristic to the underlying filtered $\mathscr{D}_{Y^{-}}$ Module $(M, F)$ of $\mathscr{M} \in \operatorname{MHM}(Y)$. Then

$$
\begin{aligned}
& \mathscr{H}^{j} f^{*} \mathscr{M}=\mathscr{H}^{-j} f^{!} \mathscr{M}=0 \quad \text { for } j \neq d \\
& W_{i} \mathscr{H}^{d} f^{*} \mathscr{M} \simeq \mathscr{H}^{d} f^{*} W_{i-d} \mathscr{M}, W_{i} \mathscr{H}^{-d} f^{!} \mathscr{M} \simeq \mathscr{H}^{-d} f^{!} W_{i+d} \mathscr{M}
\end{aligned}
$$

and the underlying filtered $\mathscr{D}_{X}$-Modules of $\mathscr{H}^{d} f^{*} \mathscr{M}, \mathscr{H}^{-d} f^{!} \mathscr{M}$ are isomorphic to $f^{*}(M, F)[d], f^{!}(M, F)[-d](c f .[19,(3.5 .1)])$.

Proof. By definition (and by $[19,(3.5 .4)]$ ) we may assume $f$ is a closed immersion and $X=\left\{x_{1}=\cdots=x_{d}=0\right\}$ (because the definition of $f^{*}, f^{!}$in $[19,(3.5 .1)]$ is compatible with DR.) If $d=-1$, the assertion follows from $[19,(3.5 .6)]$ (because it implies $\phi_{x_{1}, 1} \mathscr{M}=0$ and $N=0$ on $\psi_{x_{1}, 1} \mathscr{M}$.) In general, we can reduce to this case by factorizing $i$ (cf. the remark of 2.24 and $[19,(3.5 .4)])$.

2.26. Proposition. Let $f: X \rightarrow Y$ be as in 2.19. If $\mathscr{M} \in \operatorname{MHM}(Y)$ satisfies $\mathrm{Gr}_{i}^{W} \mathscr{M}=0$ for $i>n$ (resp. $i<n$ ), we have $\mathrm{Gr}_{i}^{W} \mathscr{H}^{j} f^{*} \mathscr{M}=0$ for $i-j>n$ (resp. $\mathrm{Gr}_{i}^{W} \mathscr{H}^{j} f^{!} \mathscr{M}=0$ for $i-j<n$ ). Let $f: X \rightarrow Y$ be as in 2.18 such that $\mathscr{H}^{j} f_{*} \mathscr{M}, \mathscr{H}^{j} f_{!} \mathscr{M}$ can be defined by (2.18.1) for $\mathscr{M} \in \mathrm{MHM}(X)^{p}$. If $\mathrm{Gr}_{i}^{W} \mathscr{M}=0$ for $i>n($ resp. $i<n)$, we have $\mathrm{Gr}_{i}^{W} \mathscr{H}^{j} f_{!} \mathscr{M}=0$ for $i-j>n\left(\right.$ resp. $\mathrm{Gr}_{i}^{W} \mathscr{H}^{j} f_{*} \mathscr{M}$ $=0$ for $i-j<n)$.

Proof. For the first assertion, we may assume $f$ is a closed immersion such that $X=\left\{g_{1}=\cdots=g_{d}=0\right\}$. Then by the remark of 2.24 , we get the long exact 
sequence:

$$
\longrightarrow \psi_{g_{d}, 1} \mathscr{H}^{j} \tilde{f}^{*} \mathscr{M} \stackrel{\text { can }}{\longrightarrow} \phi_{g_{d}, 1} \mathscr{H}^{j} \tilde{f}^{*} \mathscr{M} \longrightarrow \mathscr{H}^{j} f^{*} \mathscr{M} \longrightarrow
$$

where $\tilde{f}:\left\{g_{1}=\cdots=g_{g-1}=0\right\} \rightarrow Y$. Thus we may assume $X=\{g=0\}$ by (2.24.3). Moreover we may assume $\operatorname{Gr}_{i}^{W} \mathscr{M}=0$ for $i \neq n$ (and $\mathscr{M}$ has a strict support) by the weight spectral sequence associated to the cohomological functor $\mathscr{H}^{j} f^{*}(j \in \mathbb{Z})$. If supp $\mathscr{M} \subset X$, the assertion is trivial. If not, the assertion follows from the surjectivity of

$$
\mathrm{Gr}^{W} \text { can }: \mathrm{Gr}_{i}^{W} \psi_{g, 1} \mathscr{M} \longrightarrow \mathrm{Gr}_{i}^{W} \phi_{g, 1} \mathscr{M}
$$

and its injectivity for $i \geq n$. The assertion for $\mathscr{H}^{j} f^{!}$follows from the dual argument.

For the direct images, we may assume $f$ is an open immersion whose complement is a divisor defined by $g$, because the assertion for $f$ projective is clear by the weight spectral sequence (2.15.1). Then the assertion for $f_{*} \mathscr{M}$ follows from the proof of 2.11 (cf. (2.11.10)), and the assertion for $f_{!} \mathscr{M}$ from the duality.

2.27. Lemma. Let $f: X \rightarrow Y$ be a smooth surjective morphism of separated reduced complex analytic spaces such that the fibers of $f$ are connected and of dimension d. Assume $f$ is compactified to a projective morphism $\bar{f}: \bar{X} \rightarrow Y$ such that $\bar{X} \backslash X$ is a locally principal divisor. Then, for $\mathscr{M} \in \operatorname{MHM}(X)^{p}$ extendable to $\bar{X}$, we have

$$
\mathscr{M} \simeq \mathscr{H}^{d} f^{*} \mathscr{M}^{\prime} \quad \text { for } \mathscr{M}^{\prime} \in \operatorname{MHM}(Y)^{p}
$$

iff

$$
K \simeq f^{*} K^{\prime}[d] \quad \text { for } K^{\prime} \in \operatorname{Perv}\left(\mathbf{Q}_{Y}\right),
$$

where $K$ is the underlying perverse sheaf of $\mathscr{M}$.

Proof. Assume (2.27.2). We define

$$
\mathscr{M}^{\prime}=\mathscr{H}^{-d} \bar{f}_{*}\left(j_{*} \mathscr{M}\right) \in \operatorname{MHM}(Y)^{p}
$$

where $j: X \rightarrow \bar{X}$. Then the underlying perverse sheaf of $\mathscr{M}^{\prime}$ is canonically isomorphic to $K^{\prime}$ by the direct image of (2.27.2), because $C\left(K^{\prime}\right.$ $\left.\rightarrow f_{*} f^{*} K^{\prime}\right) \in D_{c}^{b}\left(Q_{Y}\right)^{>0}$. Therefore it is enough to show (2.27.2) induces an isomorphism in $\operatorname{MHM}(X)$, and the assertion is local on $Y$. Hence we may assume $\bar{X}, Y$ are closed subvarieties of $Z=\mathbb{P}^{n} \times U, U$ with $U$ smooth, so that $i^{\prime} \circ \bar{f}=\operatorname{pr}_{2} \circ i$, where $i: \bar{X} \rightarrow Z, i^{\prime}: Y \rightarrow U$. Let $s: Y \rightarrow X$ be a section of $f$. Using an automorphism of $\mathbb{P}^{n}$ parametrized by $Y$ (and changing $i$ ), we may assume $\operatorname{Im} s=\{p\} \times Y$ for $p \in \mathbb{P}^{n}$, so that $s$ is extended to a section $s^{\prime}$ of $\operatorname{pr}_{2}$ satisfying 
$\operatorname{Im} s^{\prime}=\{p\} \times U$. Taking hyperplanes of $\mathbb{P}^{n}$ whose intersection is $\{p\}$, we can define the natural functor

$$
s_{*}^{\prime}\left(s^{\prime}\right)^{*}: D^{b} \operatorname{MHM}(Z)^{p} \longrightarrow D_{\operatorname{lm} s^{\prime}}^{b} \operatorname{MHM}(Z)^{p}
$$

with the natural morphism: id $\rightarrow s_{*}^{\prime}\left(s^{\prime}\right)^{*}$, by the same argument as in the proof of 2.19. For $\tilde{\mathscr{M}}=i_{*} j_{*} \mathscr{M}$, we have

$$
\mathscr{H}^{j}\left(s^{\prime}\right)^{*} \tilde{\mathscr{M}} \simeq i_{*}^{\prime} \mathscr{H}^{j} s^{*} \mathscr{M}=0 \quad \text { for } j \neq-d
$$

and we get a morphism in $D^{b} F_{h}\left(\mathscr{D}_{Z}\right)$

$$
(\tilde{M}, F) \longrightarrow s_{*}^{\prime}\left(\tilde{M}_{s}, F\right)[-d]
$$

compatible with the natural morphism in $D_{c}^{b}\left(\mathbf{Q}_{\mathbf{Z}}\right)$ :

$$
i_{*} j_{*} K \longrightarrow i_{*} j_{*} s_{*} s^{*} K
$$

where $(\tilde{M}, F),\left(\tilde{M}_{s^{\prime}}, F\right)$ are the underlying filtered $\mathscr{D}$-Modules of $\tilde{\mathscr{M}}, \mathscr{H}^{-d}\left(s^{\prime}\right)^{*} \tilde{\mathscr{M}}$, and $K$ is represented by a flabby complex as in the proof of 2.19. Taking the direct image $\mathscr{H}^{-d}\left(\mathrm{pr}_{2}\right)_{*}$, we get the canonical morphism in $\operatorname{MHM}(U)$, i.e. in $M F_{h} W\left(\mathscr{D}_{U}, \mathbb{Q}\right)$ :

$$
i_{*}^{\prime} \mathscr{M}^{\prime}=\mathscr{H}^{-d}\left(\mathrm{pr}_{2}\right)_{*} \tilde{\mathscr{M}} \longrightarrow i_{*}^{\prime} \mathscr{H}^{-d} s^{*} \mathscr{M}
$$

whose underlying morphism of perverse sheaves is identified with the identity on $i_{*}^{\prime} K^{\prime}$ by $(2.27 .2)$. In fact, it preserves $W$, because ${ }^{p} \mathscr{H}^{-d} \bar{f}_{*}$ is left exact and

$$
\begin{aligned}
& W_{i}{ }^{p} \mathscr{H}^{-d} f_{*} K \simeq{ }^{p} \mathscr{H}^{-d} \bar{f}_{*}\left(W_{i+d} j_{*} K\right) \subset{ }^{p} \mathscr{H}^{-d} f_{*} W_{i+d} K \\
& W_{i}{ }^{p} \mathscr{H}^{-d} s^{*} K \simeq{ }^{p} \mathscr{H}^{-d} s^{*} W_{i+d} K,
\end{aligned}
$$

cf. 2.15, where we use an argument similar to the proof of 2.25 for the last assertion. Therefore we get the canonical isomorphism in $\operatorname{MHM}(Y)$ :

$$
\mathscr{M}^{\prime} \stackrel{\sim}{\longrightarrow} \mathscr{H}^{-d} s^{*} \mathscr{M}
$$

inducing the identity on $K^{\prime}$ by (2.27.2), for any (local) sections of $f$, and this implies the assertion.

\section{(2.f) Extensions over Closed Subspaces}

2.28. Proposition. With the notation of 2.22, let $\operatorname{MHM}(U)_{Y}^{(p)}$ be the full subcategory of $\mathrm{MHM}(U)^{(p)}$ of the objects extendable to $Y\left(\right.$ in $\left.\operatorname{MHM}(Y)^{(p)}\right)$, and $\operatorname{MHM}(U, X)_{\text {ex }}^{(p)}$ the category whose objects are $\left\{\mathscr{M}^{\prime}, \mathscr{M}^{\prime \prime}, u, v\right\}$ where $\mathscr{M}^{\prime} \in$ $\operatorname{MHM}(U)_{Y}^{(p)}, \mathscr{M}^{\prime \prime} \in \operatorname{MHM}(X)^{(p)}, u \in \operatorname{Hom}\left(\psi_{g, 1} \mathscr{M}^{\prime}, \mathscr{M}^{\prime \prime}\right), v \in \operatorname{Hom}\left(\mathscr{M}^{\prime \prime}, \psi_{g, 1} \mathscr{M}^{\prime}(-1)\right)$ such that $v u=N$, and whose morphisms are the pairs of morphisms in $\operatorname{MHM}(U)_{Y}^{(p)}$, $\operatorname{MHM}(X)^{(p)}$ compatible with $u, v$. Then the functor 


$$
\operatorname{MHM}(Y)^{(p)} \longrightarrow \operatorname{MHM}(U, X)_{e x}^{(p)}
$$

assigning $\left\{j^{-1} \mathscr{M}, \phi_{g, 1} \mathscr{M}\right.$, can, Var $\}$ to $\mathscr{M}$ is an equivalence of categories.

Proof. We define an inverse functor $\alpha$ by

$$
\alpha\left(\mathscr{M}^{\prime}, \mathscr{M}^{\prime \prime}, u, v\right)=C\left(C(u)[-1] \longrightarrow j_{!} \mathscr{M}^{\prime}\right)
$$

where $C(u) \rightarrow j_{!} M^{\prime}[1]$ is induced by the composition:

$$
\begin{aligned}
& C(u) \stackrel{(\mathrm{id}, v)}{\longrightarrow} C(N) \longleftrightarrow \xi_{g} C\left(j_{!} \mathscr{M}^{\prime} \longrightarrow j_{*} \mathscr{M}^{\prime}\right) \\
& \left.\longrightarrow j_{*} \mathscr{M}^{\prime}\right) \stackrel{\text { pr }}{\longrightarrow} j_{!} \mathscr{M}^{\prime}[1] .
\end{aligned}
$$

Here we use $2.22,2.24$ and the constructionin 2.29 below to avoid the ambiguity of the mapping cone in the derived category, i.e. $\alpha\left(\mathscr{M}^{\prime}, \mathscr{M}^{\prime \prime}, u, v\right)$ is defined by

$$
C\left(C(u)[-1] \longrightarrow C(N)[-1] \longleftarrow \xi_{g} C\left(j_{!} \mathscr{M}^{\prime} \longrightarrow j_{*} \mathscr{M}^{\prime}\right)[-1] \longrightarrow j_{!} \mathscr{M}^{\prime}\right)
$$

in the notation of 2.29. We check that $\phi_{g, 1} \alpha\left(\mathscr{M}^{\prime}, \mathscr{M}^{\prime \prime}, u, v\right)$ is canonically isomorphic to

$$
C\left(C(u)[-1] \longrightarrow \psi_{g, 1} \mathscr{M}^{\prime}\right) \simeq \mathscr{M}^{\prime \prime} \in \operatorname{MHM}_{X}(Y)^{(p)},
$$

using the construction in 2.29 below and the commutative diagram for $\tilde{\mathscr{M}}$ $=C\left(j_{!} \mathscr{M}^{\prime} \rightarrow j_{*} \mathscr{M}^{\prime}\right)[-1] \in C_{X}^{b} \operatorname{MHM}(Y)^{(p)}$ :

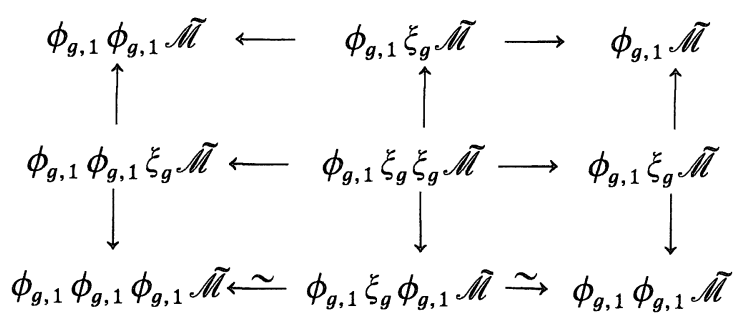

cf. the remark of 2.23. Put $\mathscr{M}=\alpha\left(\mathscr{M}^{\prime}, \mathscr{M}^{\prime \prime}, u, v\right)$. Then $j^{-1} \mathscr{M}=\mathscr{M}^{\prime}$ (hence $\left.\psi_{g, 1} \mathscr{M}=\psi_{g, 1} \mathscr{M}^{\prime}\right)$ and $\mathscr{M} \in \operatorname{MHM}(Y)^{(p)}$ because $\mathscr{H}^{j} \mathscr{M}=0$ for $j \neq 0$. For $\left(\mathscr{M}^{\prime}, \psi_{g, 1} \mathscr{M}^{\prime}\right.$, id, $\left.N\right)$ and $\left(\mathscr{M}^{\prime}, \psi_{g, 1} \mathscr{M}^{\prime}(-1), N\right.$, id), we have the canonical isomorphisms

$$
\begin{aligned}
& \alpha\left(\mathscr{M}^{\prime}, \psi_{g, 1} \mathscr{M}^{\prime}, \mathrm{id}, N\right) \simeq j_{!} \mathscr{M}^{\prime}, \\
& \alpha\left(\mathscr{M}^{\prime}, \psi_{g, 1} \mathscr{M}^{\prime}(-1), N, \mathrm{id}\right) \simeq j_{*} \mathscr{M}^{\prime}
\end{aligned}
$$

such that their $\phi_{g, 1}$ are identified with the identity on $\psi_{g, 1} \mathscr{M}^{\prime}$ and $\psi_{g, 1} \mathscr{M}^{\prime}(-1)$ by the above isomorphism (and by 2.24), and $\alpha$ and $\phi_{g, 1} \alpha$ of the morphisms: 


$$
\begin{aligned}
\left(\mathscr{M}^{\prime}, \psi_{g, 1} \mathscr{M}^{\prime}, \mathrm{id}, N\right) & \stackrel{\text { (id, } u)}{\longrightarrow}\left(\mathscr{M}^{\prime}, \mathscr{M}^{\prime \prime}, u, v\right) \\
& \stackrel{(\mathrm{id}, v)}{\longrightarrow}\left(\mathscr{M}^{\prime}, \psi_{g, 1} \mathscr{M}^{\prime}(-1), N, \mathrm{id}\right)
\end{aligned}
$$

are identified with the (natural) morphisms:

$$
\begin{aligned}
& j_{!} j^{-1} \mathscr{M} \longrightarrow \mathscr{M} \longrightarrow j_{*} j^{-1} \mathscr{M} \\
& \psi_{g, 1} \mathscr{M}^{\prime} \stackrel{u}{\longrightarrow} \mathscr{M}^{\prime \prime} \stackrel{v}{\longrightarrow} \psi_{g, 1} \mathscr{M}^{\prime}(-1)
\end{aligned}
$$

by the above isomorphisms. Therefore $\alpha$ is a right inverse. For $\mathscr{M} \in$ $\operatorname{MHM}(Y)^{(p)}$, we verify an isomorphism

$$
C\left(C\left(j_{!} j^{-1} \mathscr{M} \longrightarrow \mathscr{M}\right)[-1] \stackrel{\mathrm{pr}}{\longrightarrow} j_{!} j^{-1} \mathscr{M}\right) \simeq C\left(C(u)[-1] \longrightarrow j_{!} j^{-1} \mathscr{M}\right)
$$

by a similar argument. This shows $\alpha$ is a left inverse.

Remark. Another (but essentially equivalent) construction of an inverse functor is pointed out by Kashiwara. He uses (2.22.6) and defines it by the single complex associated to the double complex

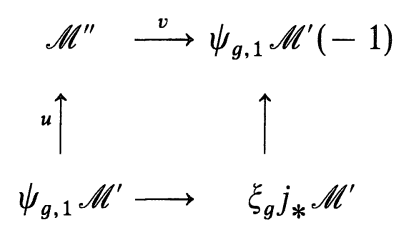

Then it is clearly a left inverse. (Problem: prove directly that it is a right inverse.)

2.29. Let $\mathscr{A}$ be an abelian category, and $\left(X_{i}, Y_{i} ; f_{i}, g_{i}\right)$ a diagram of morphisms in $C^{b}(\mathscr{A})$ :

$$
\cdots \longrightarrow Y_{i-1} \stackrel{g_{2-1}}{\longleftarrow} X_{i} \stackrel{f_{i}}{\longrightarrow} Y_{i} \stackrel{g_{i}}{\longleftarrow} X_{i+1} \longrightarrow \cdots
$$

such that $X_{i}=Y_{i}=0$ for $|i| \gg 0$. We define the cone $C\left(X_{i}, Y_{i} ; f_{i}, g_{i}\right)$ by

$$
C\left(\sum f_{i}-\sum g_{i}: \oplus X_{i} \longrightarrow \oplus Y_{i}\right) \text {. }
$$

If $f_{j}=g_{j}\left(\right.$ hence $\left.X_{j}=X_{j+1}\right)$ or $g_{j}\left(\right.$ resp. $\left.f_{j}\right)=$ id (hence $Y_{j}=X_{j+1}\left(\right.$ resp. $\left.X_{j}\right)$ ) for some $j \in \mathbb{Z}$, we have the contraction $\left(X_{i}^{\prime}, Y_{i}^{\prime} ; f_{i}^{\prime}, g_{i}^{\prime}\right)$ by

$$
\begin{gathered}
\cdots \longrightarrow Y_{j-1} \stackrel{g_{J-1}}{\longleftarrow} X_{j} \stackrel{f_{j}^{\prime}}{\longrightarrow} Y_{j+1} \stackrel{g_{J+1}}{\longleftarrow} X_{j+2} \longrightarrow \cdots \\
\text { (resp. } \left.\cdots \longleftarrow X_{j-1} \stackrel{f_{J-1}}{\longrightarrow} Y_{j-1} \stackrel{g_{j-1}^{\prime}}{\longleftarrow} X_{j+1} \stackrel{f_{j+1}}{\longrightarrow} Y_{j+1} \longleftarrow \cdots\right)
\end{gathered}
$$


such that $f_{j}^{\prime}=f_{j+1}$ or $f_{j+1} f_{j}$ (resp. $g_{j-1}^{\prime}=g_{j-1}$ or $g_{j-1} g_{j}$ ), and a canonical morphism in $C^{b}(\mathscr{A})$ :

$$
C\left(X_{i}^{\prime}, Y_{i}^{\prime} ; f_{i}^{\prime}, g_{i}^{\prime}\right) \longrightarrow C\left(X_{i}, Y_{i} ; f_{i}, g_{i}\right)
$$

defined by (id, id) or (id, $\left.f_{j}\right) \quad\left(\right.$ resp. $\left(g_{j}\right.$, id)): $X_{j}$ (resp. $\left.X_{j+1}\right) \longrightarrow X_{j} \oplus X_{j+1}$, where its restriction to the other $X_{i}, Y_{i}$ is the identity. Then (2.29.1) is a quasiisomorphism, if so is $f_{j}$ or $g_{j}$. Similarly, if $g_{j}=f_{j+1}$ or $f_{j+1}\left(\right.$ resp. $\left.g_{j}\right)=\mathrm{id}$, we have the contraction $\left(X_{i}^{\prime}, Y_{i}^{\prime}, f_{i}^{\prime}, g_{i}^{\prime}\right)$ by

$$
\begin{gathered}
\cdots \longleftarrow X_{j} \stackrel{f_{J}}{\longrightarrow} Y_{j} \stackrel{g_{j}^{\prime}}{\longleftarrow} X_{j+2} \stackrel{f_{J+2}}{\longrightarrow} Y_{j+2} \longleftarrow \cdots \\
\text { (resp. } \left.\cdots \longrightarrow Y_{j-1} \stackrel{g_{j-1}}{\longleftarrow} X_{j} \stackrel{f_{J}^{\prime}}{\longrightarrow} Y_{j+1} \stackrel{g_{J+1}}{\longleftarrow} X_{j+2} \longrightarrow \cdots\right)
\end{gathered}
$$

such that $g_{j}^{\prime}=g_{j+1}$ or $g_{j} g_{j+1}$ (resp. $f_{j}^{\prime}=f_{j}$ or $f_{j+1} f_{j}$ ) and a morphism in $C^{b}(\mathscr{A})$ :

$$
C\left(X_{i}, Y_{i} ; f_{i}, g_{i}\right) \longrightarrow C\left(X_{i}^{\prime}, Y_{i}^{\prime}, f_{i}^{\prime}, g_{i}^{\prime}\right)
$$

defined by $X_{j+1} \rightarrow 0$ and $\mathrm{id}+\mathrm{id}$ or id $+g_{j}$ (resp. $\left.f_{j+1}+\mathrm{id}\right): Y_{j} \oplus Y_{j+1} \rightarrow Y_{j}$ (resp. $Y_{j+1}$ ), which is a quasi-isomorphism, if so is $f_{j+1}$ or $g_{j}$. If $g_{j}=$ id or $f_{j}$ $=\mathrm{id}$, the composition of (2.29.1) and (2.29.2) is the identity on the cone of the contraction, and that of (2.29.2) and (2.29.1) is homotopic to the identity with the homotopy defined by id: $Y_{j} \rightarrow X_{j+1}$ or $-\mathrm{id}: Y_{j} \rightarrow X_{j}$. Let $f^{\prime}: Y_{j} \rightarrow Z$ and $g^{\prime}: Y_{j+1} \rightarrow Z$ be morphisms in $C^{b}(\mathscr{A})$ such that $f^{\prime} g_{j}=g^{\prime} f_{j+1}$. By the functoriality of the cone, we have the canonical morphisms in $C^{b}(\mathscr{A})$ :

$$
\begin{aligned}
& C\left(\cdots \longleftarrow X_{j} \stackrel{f_{J}}{\longrightarrow} Y_{j} \stackrel{g_{J}}{\longleftarrow} X_{j+1} \stackrel{f_{J+1}}{\longrightarrow} Y_{j+1} \longleftarrow \cdots\right) \\
& \longrightarrow C\left(\cdots \longrightarrow Y_{j} \stackrel{g_{j}}{\longrightarrow} X_{j+1} \stackrel{\text { id }}{\longrightarrow} X_{j+1} \stackrel{\text { id }}{\longleftarrow} X_{j+1} \stackrel{f_{j+1}}{\longrightarrow} Y_{j+1} \longleftrightarrow \cdots\right) \\
& \longrightarrow C\left(\cdots \longrightarrow Y_{j} \stackrel{\mathrm{id}}{\longleftarrow} Y_{j} \stackrel{f^{\prime}}{\longrightarrow} Z \stackrel{g^{\prime}}{\longleftarrow} Y_{j+1} \stackrel{\mathrm{id}}{\longrightarrow} Y_{j+1} \longleftarrow \cdots\right) \\
& \longrightarrow C\left(\cdots \longleftarrow X_{j} \stackrel{f^{\prime} f_{J}}{\longrightarrow} Z \stackrel{g^{\prime} g_{j+1}}{\longleftarrow} X_{j+2} \longrightarrow \cdots\right) .
\end{aligned}
$$

We have similar morphisms for $f^{\prime}: Z \rightarrow X_{j+1}$ and $g^{\prime}: Z \rightarrow X_{j}$ such that $g_{j} f^{\prime}$ $=f_{j} g^{\prime}$. If $X_{i}=Y_{i}=0$ for $i<0$ or $i>a$ and $g_{i}$ are quasi-isomorphisms for $0 \leq i$ $<a$, the cone $C\left(X_{i}, Y_{i} ; f_{i}, g_{i}\right)$ represents the mapping cone of $f_{a} g_{a-1}^{-1} \cdots g_{0}^{-1} f_{0}: X_{0} \rightarrow Y_{a}$ in $D^{b}(\mathscr{A})$ by definition.

2.30. Let $i: X \rightarrow Y$ be a closed immersion of separated reduced complex analytic spaces, $C_{X}$ the normal cone of $X$ in $Y$, and $j: Y^{*}:=Y \backslash X \rightarrow Y, j: C_{X}^{*}$ $:=C_{X} \backslash X \rightarrow C_{X}$ the natural inclusions. We shall denote also by $i$ the inclusion $X \rightarrow C_{X}$. We have the deformation of $Y$ to $C_{X}$ by 


$$
\tilde{Y}=\operatorname{Specan}_{Y}\left(\bigoplus_{n \in \mathbf{Z}} \mathscr{I}_{X}^{-n} t^{n}\right)_{\mathrm{red}}
$$

with the projection $p: \tilde{Y} \rightarrow S=$ Specan $\mathbf{C}[t]$ as is well-known, where $\mathscr{I}_{X}$ is the Ideal of $X$ in $Y$ and $\mathscr{I}_{X}^{-n}=\mathcal{O}_{\underline{Y}}$ for $n \geq 0$. Then $p^{-1}(0)_{\text {red }} \cong C_{X}$ and $p^{-1}\left(S^{*}\right) \cong Y$ $\times S^{*}$ with $S^{*}=S \backslash\{0\}$, and $\tilde{Y}$ is an (affine) open subset (over $Y$ ) of the blow-up of $Y \times S$ with center $X \times\{0\}$, i.e. the complement of the proper transform of $Y$ $\times\{0\}$. Therefore, an object of $\operatorname{MHM}\left(Y \times S^{*}\right)^{(p)}$ is extendable to $\tilde{Y}$, if it is extendable to $Y \times S$, by 2.19 and 3.21, and following Verdier, we can define the specialization functor:

$$
\operatorname{Sp}_{X}: \operatorname{MHM}(Y)^{(p)} \longrightarrow \operatorname{MHM}\left(C_{X}\right)^{(p)}
$$

by

$$
\operatorname{Sp}_{X}(M)=\psi_{p}\left(\mathscr{M} \otimes \mathbf{Q}_{S^{*}}^{H}[1]\right)
$$

Then $\mathrm{Sp}_{X}$ is exact, commutes with $\mathbf{D}$ and induces the identity on $\operatorname{MHM}(X)^{(p)}$. In particular, it induces

$$
\operatorname{Sp}_{X}: \operatorname{MHM}\left(Y^{*}\right)_{Y}^{(p)} \longrightarrow \operatorname{MHM}\left(C_{X}^{*}\right)_{C_{X}}^{(p)},
$$

where they are the full subcategories of the objects extendable to $Y$ and $C_{X}$. We shall also denote by $\mathrm{Sp}_{X}$ the induced functor:

$$
\operatorname{Sp}_{X}: D^{b} \operatorname{MHM}(Y)^{(p)} \longrightarrow D^{b} \operatorname{MHM}\left(C_{X}\right)^{(p)}
$$

If the Ideal of $X$ in $Y$ is globally generated by holomorphic functions $g_{1}, \ldots, g_{r}$, so is the Ideal of $X$ in $C_{X}$, and as in the proof of 2.19 , we can define the functors $j_{!} j^{-1}, j_{*} j^{-1}, i_{*} i^{*}, i_{*} i^{!}$in $D^{b} \operatorname{MHM}(Y)^{(p)}$ and $D^{b} \operatorname{MHM}\left(C_{X}\right)^{(p)}$, with the triangles:

$$
\begin{aligned}
& \longrightarrow j_{!} j^{-1} \mathscr{M} \longrightarrow \mathscr{M} \longrightarrow i_{*} i^{*} \mathscr{M} \stackrel{+1}{\longrightarrow} \\
& \longrightarrow i_{*} i^{!} \mathscr{M} \longrightarrow \mathscr{M} \longrightarrow j_{*} j^{-1} \mathscr{M} \stackrel{+1}{\longrightarrow}
\end{aligned}
$$

for $\mathscr{M} \in D^{b} \operatorname{MHM}(Y)^{(p)}, D^{b} \operatorname{MHM}\left(C_{X}\right)^{(p)}$ using the Cech covering associated to $\left\{g_{i} \neq 0\right\}$ or $\left\{\operatorname{gr} g_{i} \neq 0\right\}$. Then $\mathrm{Sp}$ commutes with those functors and preserves (2.30.1), because we have the commutative diagram

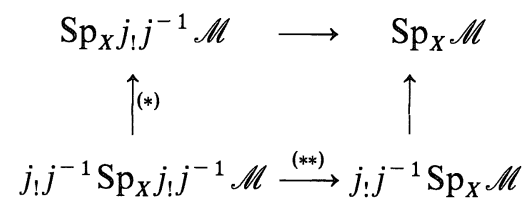

such that $(*)(* *)$ are quasi-isomorphisms. (In fact, we can reduce to the case $\mathscr{M} \in \operatorname{MHM}(Y)$, then the assertion is true for the underlying $\mathbf{Q}$-complexes.) In particular 


$$
\begin{aligned}
& \mathscr{H} \operatorname{om}_{D^{b} M H M(Y)^{(p)}}\left(j_{!} j^{-1} \mathscr{M}, \mathscr{M}^{\prime}\right)=0 \\
& \mathscr{H} \operatorname{om}_{D^{b} M H M\left(C_{X}\right)^{(p)}}\left(\operatorname{Sp}_{X} j_{!} j^{-1} \mathscr{M}, \operatorname{Sp}_{X} \mathscr{M}^{\prime}\right)=0
\end{aligned}
$$

for $\mathscr{M} \in D^{b} \operatorname{MHM}(Y)^{(p)}, \mathscr{M}^{\prime} \in D_{X}^{b} \operatorname{MHM}(Y)^{(p)}$, because we can reduce to the case $\mathscr{M}^{\prime} \in \mathrm{MHM}(X)^{(p)}$ and any resolution of $j_{!} j^{-1} \mathscr{M}, \mathrm{Sp}_{X} j_{!} j^{-1} \mathscr{M}$ may be replaced by its Čech complex (representing the functor $j_{!} j^{-1}$ as above). Therefore we get

$$
\mathscr{H} o m_{D^{b} M M(Y)^{(p)}}\left(\mathscr{M}, \mathscr{M}^{\prime}\right) \stackrel{\sim}{\longrightarrow} \mathscr{H} \circ m_{D^{b} M H M\left(C_{X}\right)^{(p)}}\left(\operatorname{Sp}_{X} \mathscr{M}, \mathrm{Sp}_{X} \mathscr{M}^{\prime}\right)
$$

for $\mathscr{M}, \mathscr{M}^{\prime}$ as above, because we can replace $\mathscr{M}$ by $i_{*} i^{*} \mathscr{M}$ (i.e. we have proved essentially the adjunction for $i_{*} i^{*}$.) Thus we get the following proposition by the same argument as in [23], because the assertion is local. In fact, the polarizability of $\mathrm{Gr}_{i}^{W} \mathscr{M}$ follows from 3.21 (if the strict support is not contained in $X$ ) and from $\operatorname{Sp}_{X}=$ id on $\operatorname{MHM}(X)$ (otherwise).

2.31. Proposition. With the notation of 2.30 , let $\operatorname{MHM}\left(Y^{*}, C_{X}\right)_{g 1}^{(p)}$ be the category whose objects are $\left(\mathscr{M}^{\prime}, \mathscr{M}^{\prime \prime} ; \alpha\right)$ where $\mathscr{M}^{\prime} \in \operatorname{MHM}\left(Y^{*}\right)_{Y}^{(p)}, \mathscr{M}^{\prime \prime} \in$ $\operatorname{MHM}\left(C_{X}\right)^{(p)}$ and $\alpha: \operatorname{Sp}_{X} \mathscr{M}^{\prime} \simeq j^{-1} \mathscr{M}^{\prime \prime}$, and whose morphisms are the pairs of morphisms in $\operatorname{MHM}\left(Y^{*}\right)_{Y}^{(p)}$ and $\operatorname{MHM}\left(C_{X}\right)^{(p)}$ compatible with $\alpha$. Then the natural functor

$$
\operatorname{MHM}(Y)^{(p)} \longrightarrow \operatorname{MHM}\left(Y^{*}, C_{X}\right)_{g 1}^{(p)}
$$

assigning $\left(j^{-1} M, \mathrm{Sp}_{X} M\right.$; id) to $\mathscr{M} \in \mathrm{MHM}(Y)^{(p)}$ is an equivalence of categories.

2.32. Let $X$ be a complex manifold and $q: E \rightarrow X$ an analytic vector bundle of rank $r$. Let $q^{\prime}: E^{\vee} \rightarrow X$ be the dual bundle, and put $\widetilde{E}=E \times{ }_{X} E^{\vee}$ with the natural projections $p: \widetilde{E} \rightarrow E^{\vee}$ and $p^{\prime}: \widetilde{E} \rightarrow E$. Then we have a natural holomorphic function $g$ on $\widetilde{E}$ induced by the natural pairing on the fibers. Let $E^{*}, \widetilde{E}^{*}$ be the complement of the zero section of $q, p$ with the inclusion $j: E^{*}$ $\rightarrow E, j: \widetilde{E}^{*} \rightarrow \widetilde{E}$. For $\mathscr{M} \in \operatorname{MHM}(E)^{(p)}$ we define

$$
\Phi(\mathscr{M})=\varphi_{g, 1} \mathscr{H}^{r}\left(p^{\prime}\right)^{*} \mathscr{M} \in \operatorname{MHM}_{g^{-1}(0)}(\widetilde{E})^{(p)} .
$$

Then $\Phi(\mathscr{M})$ is extendable to $E \times \bar{E}^{\vee}$, where $\bar{E}^{\vee} \rightarrow X$ is the natural projectification (i.e. compactification) of $q^{\prime}$, because the closure of the graph of $g$ in $E \times{ }_{X} \bar{E}^{\vee} \times \mathbb{C}$ is analytic. For $\mathscr{M}^{\prime} \in \operatorname{MHM}\left(E^{*}\right)_{E}^{(p)}$ (i.e. extendable to $E$ ) we set

$$
\Phi_{!}\left(\mathscr{M}^{\prime}\right)=\Phi\left(\mathscr{H}^{0} j_{!} \mathscr{M}^{\prime}\right), \quad \Phi_{*}\left(\mathscr{M}^{\prime}\right)=\Phi\left(\mathscr{H}^{0} j_{*} \mathscr{M}^{\prime}\right) .
$$

Then we have a natural morphism

$$
w: \Phi_{!}\left(\mathscr{M}^{\prime}\right) \rightarrow \Phi_{*}\left(\mathscr{M}^{\prime}\right)
$$

induced by $\mathscr{H}^{0} j_{!} \mathscr{M} \rightarrow \mathscr{H}^{0} j_{*} \mathscr{M}$. If $\mathscr{M}^{\prime}=j^{-1} \mathscr{M}$, we have a factorization 


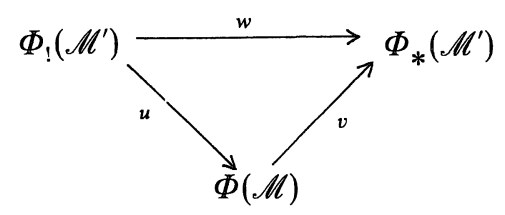

induced by $\mathscr{H}^{0} j_{!} \mathscr{M}^{\prime} \rightarrow \mathscr{M} \rightarrow \mathscr{H}^{0} j_{*} \mathscr{M}^{\prime}$. Here Ker, Coker of $u, v, w$ are the pullbacks by $p^{\prime}$ of Ker, Coker of the corresponding morphisms between $\mathscr{H}^{0} j_{!} \mathscr{M}^{\prime}, \mathscr{M}, \mathscr{H}^{0} j_{*} \mathscr{M}^{\prime}$; in particular, they are supported in the zero section of $p$ or $q$

Conversely, for $\mathscr{M}^{\prime} \in \operatorname{MHM}\left(E^{*}\right)_{E}^{(p)}$ and $\mathscr{M}^{\prime \prime} \in \operatorname{MHM}_{g^{-1}(0)}(\widetilde{E})^{(p)}$ with a commutative diagram

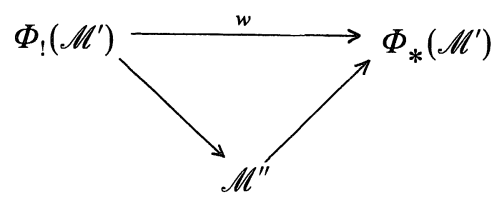

we have uniquely $\tilde{\mathscr{M}} \in \mathrm{MHM}(\widetilde{E})^{(p)}$ with isomorphisms

$$
\phi_{g, 1} \tilde{\mathscr{M}} \simeq \mathscr{M}^{\prime \prime}, j^{-1} \tilde{\mathscr{M}}=\mathscr{H}^{r}\left(p^{\prime}\right)^{*} \mathscr{M}^{\prime}
$$

such that $\phi_{g, 1}$ of $\mathscr{H}^{0} j_{!} j^{-1} \tilde{\mathscr{M}} \rightarrow \tilde{\mathscr{M}} \rightarrow \mathscr{H}^{0} j_{*} j^{-1} \tilde{\mathscr{M}}$ is identified with (2.32.2), cf. 2.28 and 2.24. Therefore the problem is when $\tilde{\mathscr{M}}$ is the pull-back of $\mathscr{M} \in$ $\operatorname{MHM}(E)^{(p)}$, and we can restrict $\widetilde{E}$ to an open subset $p^{-1}(U)$ where $U$ is an open set of $E^{\vee}$ such that $q^{\prime}(U)=X$ and the complement of $U$ in the natural projectification of $q^{\prime}$ is analytic, because we are interested in the extension of $\mathscr{M}^{\prime}$ to $E$, and not that of $\left(p^{\prime}\right)^{-1} \mathscr{M}^{\prime}$ to $\widetilde{E}$. By 2.27 , the problem is reduced to that for the underlying perverse sheaves in the polarizable case, then the answer is known by MacPherson, Gabber, Malgrange [17][23] (cf. also Malgrange, $B$.: Variations généralisée, Astérisque 130, p.237-239), if the underlying perverse sheaves of $\Phi_{!}\left(\mathscr{M}^{\prime}\right), \Phi_{*}\left(\mathscr{M}^{\prime}\right), \mathscr{M}^{\prime \prime}$ (on $p^{-1}(U)$ ) are the local systems shifted by $\operatorname{dim} U$ on the zero section $U$ and the underlying perverse sheaf of $\mathscr{M}^{\prime}$ is topologically trivial along $X$. Therefore we get an analogue of a result of MacPherson-Vilonen [17] as follows:

Let $Y$ be a separated reduced analytic space, and $X$ a Zariski locally closed smooth analytic subset (i.e. $\bar{X}$ and $\partial X=\bar{X} \backslash X$ are analytic) such that $\mathscr{I}_{X} / \mathscr{I}_{X}^{2}$ is locally free, where $\mathscr{I}_{\bar{X}}$ is the Ideal of $\bar{X}$ and $\mathscr{I}_{X}$ is its restriction to $X$. Then we define

$$
E_{\bar{X}}=\operatorname{Specan}_{Y}\left(\oplus S^{i}\left(\mathscr{I}_{\bar{X}} / \mathscr{I}_{\bar{X}}^{2}\right)\right)_{\mathrm{red}}
$$

where $S^{i}$ is the $i^{\text {th }}$ symmetric tensor, so that its restriction $E_{X}$ to $X$ is a vector 
bundle and $C_{\bar{X}}, C_{X}$ are closed subspaces of $E_{\bar{X}}, E_{X}$. Similarly $E_{X}^{v}$ the dual bundle of $E_{X}$ is extended using the dual of $\mathscr{I}_{\bar{X}} / \mathscr{I}_{\bar{X}}^{2}$, so that $g$ is meromorphically extended to the closure of $\widetilde{E}_{X}=E_{X} \times{ }_{X} E_{X}^{\vee}$ in $\left(E_{\bar{X}} \times{ }_{\bar{X}} E_{\bar{X}}^{\vee}\right)_{\text {red }}$ and $E_{\bar{X}}^{\vee} \rightarrow \bar{X}$ is naturally compactified to a projective morphism $\bar{E}_{\bar{X}}^{\vee} \rightarrow \bar{X}$. We define $\operatorname{MHM}(Y \backslash \partial X)_{Y, X}^{p}$ to be the full subcategory of $\operatorname{MHM}(Y \backslash \partial X)_{Y}^{p}$ (i.e. extendable to $Y)$ such that the underlying perverse sheaf $K$ of its object satisfies:

there is a Whitney stratification of $\operatorname{supp~} \mathrm{Sp}_{X} K$ compatible with $\mathrm{Sp}_{X} K$ such that each stratum is conical (invariant by $\mathbb{C}^{*}$ ), Zariski locally closed in $E_{\bar{X}}$, and smooth over $X$.

In particular, $\operatorname{Sp}_{X} K$ is locally trivial along $X$. Let $\operatorname{MHM}(Y \backslash \bar{X})_{Y, X}^{p}$ be the full subcategory of $\operatorname{MHM}(Y \backslash \bar{X})_{Y}^{p}$ such that, for the underlying perverse sheaf $K^{\prime}$ of its object, $\operatorname{Sp}_{X} K^{\prime} \in \operatorname{Perv}\left(\mathbb{Q}_{C_{X}^{*}}\right)$ is locally trivial along $X$ in the sense of (2.32.3) (i.e. ${ }^{p} \mathscr{H}^{0} j_{!} K$ (or equivalently ${ }^{p} \mathscr{H}^{0} j_{*} K$ ) satisfies (2.32.3)). We define

$$
\operatorname{MHM}_{g s}\left(E_{X}^{\vee}\right)_{\bar{E}}^{p} \overline{\bar{x}}=\underline{\varliminf_{U}} \operatorname{MHM}_{s}(U)_{\overline{E_{\bar{X}}}}^{p}
$$

where $U$ runs over the open sets of $E_{X}^{\vee}$ such that $\bar{E}_{\bar{X}}^{\vee} \backslash U$ is analytic and $q^{\prime}(U)$ $=X$, and $\operatorname{MHM}_{s}(U)_{\overline{E_{\bar{X}}}}^{p}$ is the full subcategory of $\operatorname{MHM}(U)_{\overline{\bar{E}_{\bar{X}}}}^{p}$ such that the underlying perverse sheaf of its object is a local system shifted by $\operatorname{dim} U$ ( $s$ is for smooth). By definition, $\Phi_{!} \operatorname{Sp}_{X} \mathscr{M}$ and $\Phi_{*} \operatorname{Sp}_{X} \mathscr{M}$ belong to $\operatorname{MHM}_{g s}\left(E_{X}^{\vee}\right)_{\bar{E}_{\bar{X}}^{v}}^{p}$. In fact we take for $U$ the complement of the union of the closure of $T_{S_{\alpha}}^{*} E_{X}$ for $S_{\alpha} \not \subset X$, where $\left\{S_{\alpha}\right\}$ is the stratification in (2.32.3) and $E_{X}^{\vee}$ is identified with $T_{X}^{*} E_{X}$. Let $\operatorname{MHM}\left(Y \backslash \bar{X} ; E_{X}^{\vee}\right)_{Y, X ; \bar{E}_{\bar{X}}^{\vee}}^{p}$ be the category whose objects are $\left(\mathscr{M}^{\prime}, \mathscr{M}^{\prime \prime}, u, v\right)$ where $\mathscr{M}^{\prime} \in \operatorname{MHM}(Y \backslash \bar{X})_{Y, X}^{p}, \mathscr{M}^{\prime \prime} \in \mathrm{MHM}_{g s}\left(E_{X}^{\vee}\right)_{\bar{E}}^{p}, u: \Phi_{\frac{\bar{x}}{}}, \operatorname{Sp}_{X} \mathscr{M}^{\prime}$ $\rightarrow \mathscr{M}^{\prime \prime}$ and $v: \mathscr{M}^{\prime \prime} \rightarrow \Phi_{*} \mathrm{Sp}_{X} \mathscr{M}^{\prime}$ are morphisms in $\mathrm{MHM}_{g s}\left(E_{X}^{\vee}\right)_{\bar{E}}^{\underline{\underline{v}}}$ factorizing the natural morphism $\Phi_{!} \mathrm{Sp}_{X} \mathscr{M}^{\prime} \rightarrow \Phi_{*} \mathrm{Sp}_{X} \mathscr{M}^{\prime}$, such that the monodromy of the underlying local system of $\mathscr{M}^{\prime \prime}$ restricted to each fiber of $U \rightarrow X$ is described by $u, v$ and the "variation" of Gabber, Malgrange, cf. [17]. (Note that the last condition implies $\mathscr{M}^{\prime \prime}$ can be defined over $U$ as above (depending only on $\mathscr{M}^{\prime}$ ) using the intermediate direct image.) Then the natural functor induces an equivalence of categories :

$$
\operatorname{MHM}(Y \backslash \partial X)_{Y, X}^{p} \rightarrow \operatorname{MHM}\left(Y \backslash \bar{X} ; E_{X}^{\vee}\right)_{Y, X ; \bar{E}_{\bar{X}}^{\vee}}^{p} .
$$

In fact the assertion is already proved forgetting the extendability condition (because we may assume $E_{X} \backslash U$ is a divisor by the same argument as above.) Then we can use the following facts:

i) For $\mathscr{M}^{\prime} \in \operatorname{MHM}(Y \backslash \bar{X})_{Y}^{p}, \mathscr{M}^{\prime \prime} \in \operatorname{MHM}\left(C_{X}\right)_{C_{\bar{X}}}^{p} \quad$ with an isomorphism $\left.\left.\mathrm{Sp}_{\bar{X}} \mathscr{M}^{\prime}\right|_{C_{X}^{*}} \simeq \mathscr{M}^{\prime \prime}\right|_{C_{X}^{*}}, \mathscr{M}^{\prime \prime}$ has an extension to $C_{\bar{X}}$ such that the isomorphism is extended to $C_{\bar{X}}^{*}$ (for example, use $\mathscr{H}^{0} j_{!} j^{-1}$ for the inclusions of $C_{X}^{*}, C_{X}, C_{\bar{X}}^{*}$ into $\left.C_{\bar{X}}\right)$. Therefore there exists $\mathscr{M} \in \operatorname{MHM}(Y)^{p}$ such that $\left.\mathscr{M}\right|_{Y \backslash \bar{X}} \simeq \mathscr{M}^{\prime},\left.\operatorname{Sp}_{X} \mathscr{M}\right|_{Y-i X}$ 
$\simeq \mathscr{M}^{\prime \prime}$

ii) By the construction in the proof, 2.28 holds adding the extendability condition to $\bar{Y}$ for a Zariski open immersion $\bar{j}: Y \rightarrow \bar{Y}$ such that $g$ is extended to $\bar{Y}$. (For example, apply the functor $\mathscr{H}^{0} \bar{j}_{!}$to each component in the definition of the inverse functor, and take the zeroth cohomology.) We apply it to the inclusion

$$
p^{-1}(U) \times \mathbb{C} \longrightarrow\left(E_{\bar{X}} \times \bar{X}_{\bar{X}} \bar{E}_{\bar{X}}^{\vee}\right)_{\mathrm{red}} \times \mathbf{C},
$$

where $p: \widetilde{E}_{X} \rightarrow E_{X}^{\vee}$, and to the pull-back of the Modules on $\left(E_{\bar{X}} \times \bar{X}_{\bar{X}} \bar{E}_{\bar{X}}^{\vee}\right)_{\text {red }}$ by the projection from the closure of the graph of $g$.

iii) In 2.27, the addertion holds adding the extendability condition; i.e. for $\tilde{f}: \tilde{X}$ $\rightarrow \tilde{Y}$ a projective morphism extending $\tilde{f}$ so that $\tilde{f}^{-1}(Y)=\bar{X}, \mathscr{M}^{\prime}$ is extendable to $\tilde{Y}$ iff $\mathscr{M}$ is extendable to $\tilde{X}$. We apply this to the projection from the closure of the graph of $g$ in $\left(E_{\bar{X}} \times{ }_{\bar{X}} E_{\bar{X}}\right)_{\text {red }} \times \mathbb{P}^{1}$ onto $E_{\bar{X}}$.

\section{(2.g) Kodaira Vanishing}

2.33. Proposition. Let $Z$ be a (reduced) irreducible projective variety with an ample invertible sheaf $L$, and $i: Z \rightarrow X=\mathbf{P}^{r}$ the embedding by $L^{m}$ for some positive integer $m$. Then for $\mathscr{M}=((M, F), K ; W) \in \operatorname{MHM}_{Z}(X)^{p}\left(\right.$ or $\left.\operatorname{MH}_{Z}(X, n)^{p}\right)$, $\mathrm{Gr}_{p}^{F} \mathrm{DR}_{X}(M, F)\left(c f .[19, \S 2.2]\right.$ where $\mathrm{DR}_{X}$ is denoted by $\left.\tilde{\mathrm{DR}}\right)$ belongs to $D^{b}\left(\mathcal{O}_{Z}\right)$ and it is independent of the embedding of $Z$ in a complex manifold. Moreover we have the Kodaira vanishing:

$$
\begin{array}{lr}
H^{i}\left(Z, \mathrm{Gr}_{p}^{F} \mathrm{DR}_{X}(M, F) \otimes L\right)=0 & \text { for } i>0 \\
H^{i}\left(Z, \mathrm{Gr}_{p}^{F} \mathrm{DR}_{X}(M, F) \otimes L^{-1}\right)=0 & \text { for } i<0 .
\end{array}
$$

Proof. Because $(M, F)$ is regular and quasi-unipotent along any (locally defined) $g, \mathrm{Gr}_{p}^{F} M$ is an $\mathcal{O}_{Z}$-Module by $[19,(3.2 .6)]$ and we get the first assertion, because $\operatorname{Gr}^{F} \mathrm{DR}_{X}(M, F)$ is uniquely determined by $\operatorname{Gr}^{F} M$. We verify the independence of embedding using the cartesian product as in 2.1 , because the direct image is compatible with $\mathrm{DR}$ and $\mathrm{Gr}^{F}$ (cf. $[19, \S 2.3]$ ). Then we may assume $m \geq 2$ for the Kodaira vanishing. Here we may assume also $\mathscr{M} \in \mathrm{MH}_{Z}(X, n)^{p}$, because $\mathrm{Gr}^{F} \mathrm{DR}$ is exact. It is enough to show the second assertion, because $\mathscr{M}$ is self dual up to a Tate twist and the dual in $D_{\text {coh }}^{b} F^{f}\left(\mathcal{O}_{X}\right.$, Diff $)$ is compatible with the dual in $D_{\text {coh }}^{b}\left(\mathcal{O}_{X}\right)$ by the functor $\mathrm{Gr}^{F}$, cf. $[19, \S 2.4]$.

Let $Y$ be a generic hyperplane of $X$, strictly non-characteristic to $(M, F)$, and $s$ a section of $\Gamma\left(Z, L^{m}\right)\left(\simeq \operatorname{Hom}\left(L^{-m}, \mathcal{O}_{Z}\right)\right)$ such that $s^{-1}(0)=Y \cap Z$. Then we have a finite covering

$$
\pi: \tilde{Z}=\operatorname{Specan}_{Z}\left(\oplus_{0 \leq i<m} L^{-i}\right) \rightarrow Z
$$


ramified along $Y \cap Z$, cf. the remark below. Let $j: U=X \backslash Y \rightarrow X$ be the natural inclusion. Set

$$
\begin{aligned}
& j_{*} j^{-1} \mathscr{M}=\left((M(* Y), F), j_{*} j^{*} K ; W\right) \in \mathrm{MHW}_{Z}(X)^{p} \\
& \tilde{M}=(\tilde{M}, F, \tilde{K})=\operatorname{Coker}\left(\mathscr{M} \longrightarrow \pi_{*} \pi^{*} \mathscr{M}\right) \in \mathrm{MH}_{Z}(X, n)^{p} \\
& \tilde{L}=\operatorname{Coker}\left(\mathcal{O}_{Z} \longrightarrow \pi_{*} \mathcal{O}_{\tilde{Z}}\right) \in M\left(\mathcal{O}_{Z}\right)
\end{aligned}
$$

so that $L^{-1}$ is a direct factor of $\tilde{L}$, where $\pi^{*} \mathscr{M}$ can be defined as in 2.19 and 2.25 (because $\pi$ is non-characteristic) or $\pi_{*} \pi^{*} \mathscr{M}$ can be regarded as the unique extension to $Z$ of its restriction to the smooth open set $U^{\prime}$ of $Z$ where $\pi$ is étale. Any way $\mathscr{M}$ is a direct factor of $\pi_{*} \pi^{*} \mathscr{M}$ and we have a natural injection $\mathscr{M} \rightarrow \pi_{*} \pi^{*} \mathscr{M}$ (induced by its restriction to $U^{\prime}$ ). As for $j_{*} j^{-1} \mathscr{M}$, we have an exact sequence

$$
0 \longrightarrow \mathscr{M} \longrightarrow j_{*} j^{-1} \mathscr{M} \longrightarrow \mathscr{H}^{1} i^{1} \mathscr{M} \longrightarrow 0
$$

so that $\mathscr{H}^{1} i^{1} \mathscr{M} \in M H_{Z \cap Y}(Y, n+1)^{p}$ by the non-charactericity, where $i: Y \rightarrow X$, cf. 2.11 and $[19,(3.5 .9)]$. By $[19,(5.3 .1)]$ (and 2.15), $\mathbf{R} \Gamma\left(X, \mathrm{DR}_{X}(\tilde{M}, F)\right)$ is strict, i.e. $H^{i}\left(Z, \operatorname{Gr}_{p}^{F} \mathrm{DR}_{X} \tilde{M}\right)$ is a sub-quotient of $H^{i}\left(Z, \mathrm{DR}_{X} \tilde{M}\right) \cong H^{i}(Z, \tilde{K} \otimes \mathbb{C})$, and we have

$$
H^{i}(Z, \widetilde{K})=H^{i}\left(Z, j_{!} j^{-1} \widetilde{K}\right)=H^{i}\left(Z, j_{*} j^{-1} \widetilde{K}\right)
$$

by the non-charactericity. Therefore they are zero for $i \neq 0$ by the weak Lefschetz theorem, and the assertion is reduced to the isomorphism:

$$
\mathrm{Gr}_{p}^{F} \tilde{M} \cong \mathrm{Gr}_{p}^{F} M(* Y) \otimes \tilde{L}
$$

by induction on $\operatorname{dim} Z$ using (2.33.1). For $z \in Z$, we have an arbitrary small neighborhood $U_{z}$ of $z$ in $X$ such that the restriction of $\pi$ to $U_{z} \cap Z$ is uniquely extended (up to a unique isomorphism) to a covering over $U_{z}$ ramified along $Y \cap U_{z}$. In fact it is clear if $z \notin Y$ : otherwise we use a local topological trivialization: $(X, Z) \cong(Y, Y \cap Z) \times D^{2}$ near $z$ and take a contractible neighborhood of $z$ in $Y$ such that the contraction is compatible with $Y \cap Z$. Therefore we may assume $\pi$ is extended to $\pi: \tilde{X} \rightarrow X$ ramified cyclically over $Y$ (locally, i.e. by restricting $X$ ), and it is enough to show the canonical isomorphism

$$
\left.\left.(\tilde{M}, F)\right|_{U} \cong((M, F) \otimes \tilde{L})\right|_{U}
$$

is extended (uniquely) to the isomorphism of filtered right $\mathscr{D}_{X}$-Modules

$$
(\tilde{M}, F) \cong(M(* Y), F) \otimes \tilde{L},
$$

where $\tilde{L}$ denotes also $\operatorname{Coker}\left(\mathcal{O}_{X} \rightarrow \pi_{*} \mathcal{O}_{\tilde{X}}\right)$. Here the $\mathscr{D}_{X}$-Module structure of the right hand side is given by 


$$
M(* Y) \bigotimes_{\mathcal{O}_{\mathbf{X}}} \tilde{L} \cong M(* Y) \bigotimes_{\mathcal{O}_{\mathbf{X}}(* Y)} \tilde{L}(* Y),
$$

where $(* Y)$ denotes the localization along $Y$ and $\tilde{L}(* Y)$ is a regular holonomic left $\mathscr{D}_{X}$-Module. Then both sides of (2.33.3) are regular holonomic Modules such that the action of a local equation $t$ of $Y$ is bijective. Therefore we get (2.33.3) forgetting $F$ by [14][18]. As for $F$, we consider the filtration $V$ along $Y$. We have

$$
\begin{aligned}
& \operatorname{Gr}_{\alpha}^{V} M(* Y)=0 \quad \text { for } m \notin \mathbf{Z}, \\
& \operatorname{Gr}_{V}^{\alpha} \widetilde{L}(* Y)=0 \quad \text { for } \alpha m \notin \mathbf{Z} \text { or } \alpha \in \mathbf{Z} \\
& V^{>0} \tilde{L}(* Y)=\widetilde{L}
\end{aligned}
$$

where $V$ is decreasing on left Modules so that $t \partial_{t}-\alpha$ is nilpotent on $\operatorname{Gr}_{V}^{\alpha} \tilde{L}(* Y)$. More precisely we have (locally) a unique decomposition

$$
\widetilde{L}=\bigoplus_{0<i<m} L_{i}
$$

such that $\mathrm{Gr}_{V}^{\alpha} L_{i}=0$ for $\alpha-i / m \notin \mathbb{Z}$ and $L_{i}=V^{i / m} L_{i}(* Y)$. Then

$$
\mathrm{Gr}_{\alpha}^{V}\left(M(* Y) \otimes L_{i}\right)=\left(\mathrm{Gr}_{\alpha+i / m}^{V} M(* Y)\right) \otimes L_{i}=0
$$

for $\alpha+i / m \notin \mathbb{Z}$ and

$$
F_{P} V_{k-i / m}\left(M(* Y) \otimes L_{i}\right)=\left(F_{P} V_{k} M(* Y)\right) \otimes L_{i}
$$

for $k \in \mathbb{Z}$. Therefore $(M(* Y), F) \otimes L_{i}$ (and hence $\left.(M(* Y), F) \otimes \tilde{L}\right)$ is regular and quasi-unipotent along $Y$ (because so is $(M(* Y), F)$ and $[19,(3.2 .3 .2)]$ is satisfied), and we get $(2.33 .3)$ by $[19,(3.2 .2)]$ because $\left.\operatorname{Gr}_{0}^{V}\left(M(* Y) \otimes L_{i}\right)=0\right)$.

2.34. Remarks. 1) If $Z$ is smooth and $\mathscr{M}=\mathbf{Q}_{Z}^{H}\left[d_{Z}\right]$, the above vanishing is expressed by

$$
\begin{aligned}
& H^{q}\left(Z, \Omega_{Z}^{p} \otimes L\right)=0 \text { for } p+q>\operatorname{dim} Z \\
& H^{q}\left(Z, \Omega_{Z}^{p} \otimes L^{-1}\right)=0 \text { for } p+q<\operatorname{dim} Z,
\end{aligned}
$$

i.e. the Kodaira-Nakano vanishing. If $\mathscr{M}=\mathscr{H}^{j} f_{*}\left(\mathbf{Q}_{Y}^{H}\left[d_{Y}\right]\right)$ with $f: Y \rightarrow Z$ projective such that $Y$ smooth, it implies (for $p=-\operatorname{dim} Y$ ):

$$
H^{i}\left(Z, R^{j} f_{*} \omega_{Y} \otimes L\right)=0 \text { for } i>0,
$$

i.e. the Ohsawa-Kollár vanishing. In fact, for a projective morphism of complex manifolds $f: X \rightarrow Y$ and for $\mathscr{M}=(M, F, K) \in \mathrm{MH}_{Z}(X)^{p}$ with $p_{0}=\min$ $\left\{p \in \mathbb{Z}: \operatorname{Gr}_{p}^{F} M \neq 0\right\}$, we have

$$
\begin{aligned}
& F_{p_{0}}\left(f_{*}(M, F)\right)=R f_{*} F_{p_{0}} M \text { in } D^{b}\left(\mathcal{O}_{Y}\right) \\
& F_{p_{0}} \mathscr{H}^{j} f_{*}(M, F)=R^{j} f_{*} F_{p_{0}} M
\end{aligned}
$$


by the strictness of $f_{*}(M, F)[19,(5.3 .1)]$. Moreover for the canonical decomposition:

$$
\mathscr{H}^{j} f_{*} \mathscr{M}=\mathscr{M}_{1}^{j} \oplus \mathscr{M}_{2}^{j}
$$

such that $\mathscr{M}_{1}^{j} \in M H_{Z^{\prime}}(Y, n+j)^{p}$ (where $Z^{\prime}=f(Z)$ is assumed irreducible) and $\operatorname{supp} \mathscr{M}_{2}^{j} \varsubsetneqq Z^{\prime}$, we have $F_{p_{0}} M_{2}^{j}=0$, where $\left(M_{2}^{j}, F\right)$ is the underlying filtered $\mathscr{D}$ Module. (In fact, this follows from the commutativity of $f_{*}$ and $\psi, \phi$ and the surjectivity of

$$
\text { can }: \psi_{g, 1} \mathscr{M} \longrightarrow \phi_{g, 1} \mathscr{M}
$$

for $g^{-1}(0) \not \supset Z$.) This implies some conjecture by Kollár, combined with the results in $\S 3$.

2) It was first remarked by Ramanujan that the Kodaira vanishing can be reduced to the weak Lefschetz theorem using Hodge structures. His idea was further developed by Guillén-Navarro-Puerta, Steenbrink, Esnault-Viehweg, etc. For example, we can deduce the result of Guillén-Navarro-Puerta as follows.

Let $Z$ be as in 2.33 and $a: X . \rightarrow Z$ be a simplicial (or cubic) resolution of $Z$, such that each $X_{i}$, is smooth, and projective over $Z$. Then the filtered de Rham complex $\left(\underline{\Omega}_{Z}^{\circ}, F\right)$ over $\mathbb{C}_{Z}$ is defined by $R a_{*}\left(\Omega_{X}^{\circ}, F\right)$, which has a filtration $W$ such that $\mathrm{Gr}_{-i}^{W}\left(\underline{\Omega}_{Z}^{\circ}, F\right) \cong R\left(a_{i}\right)_{*}\left(\Omega_{\dot{X}_{i}}^{\cdot}, F\right)[-i]$. We can regard $\left(\underline{\Omega}_{Z}^{*}, F\right)$ as an object of $D_{\text {hol }}^{b} F\left(\mathcal{O}_{X}\right.$, Diff). Then $\mathrm{DR}^{-1}\left(\Omega_{Z}^{\circ}, F\right)$ is strict and its $j^{\text {th }}$ cohomology underlies an object of $\mathrm{MHW}(X)^{p}$ with the weight filtration induced by $\mathscr{H}^{j} \mathrm{DR}^{-1} W[j]$, because $\mathrm{DR}^{-1} \mathrm{Gr}_{-i}^{W}\left(\Omega_{\mathrm{Z}}^{*}, F\right)$ is strict and its $j^{\text {th }}$ cohomology underlies a polarizable Hodge Module of weight $j-i$ by $[19,(5.3 .1)]$, so that the weight spectral sequence degenerates at $E_{2}$ as in the proof of 2.15. (Note that this implies Du Bois' well-definedness of $\left(\underline{\Omega}_{\bar{Z}}^{*}, F\right)$ combined with some result on simplicial (or cubic) resolution.) In particular

$$
\mathscr{H}^{j} \mathrm{Gr}_{F}^{p} \underline{\Omega}_{Z}^{\circ}=0 \text { for } j<p \text { or } j>\operatorname{dim} Z,
$$

because ${ }^{p} \mathscr{H}^{j} \mathbb{C}_{Z}=0$ for $j>\operatorname{dim} Z$ and $\left(\underline{\Omega}_{Z}^{\circ}, F\right) \cong \widetilde{\mathrm{DR}} \widetilde{\mathrm{DR}}{ }^{-1}\left(\underline{\Omega}_{Z}^{\circ}, F\right)$, cf. [19, $\S 2.2]$. (Here the assertion for $j<p$ is clear by definition.) By the same reason, 2.33 implies

$$
H^{j}\left(Z, \mathrm{Gr}_{F}^{p} \underline{\Omega}_{Z}^{\circ} \otimes L\right)=0 \text { for } j>\operatorname{dim} Z .
$$

3) Let $X$ be a separated reduced complex analytic space, and $L$ an invertible sheaf on $X$ with $s \in \Gamma\left(X, L^{-m}\right)=\operatorname{Hom}\left(L^{m}, \mathcal{O}_{X}\right)$ for $m \geq 2$ such that $X \backslash s^{-1}(0)$ is dense in $X$. Then we have a finite morphism

$$
\pi: X^{\prime}=\operatorname{Specan}_{X}\left(\oplus_{0 \leq i<m} L^{i}\right) \longrightarrow X
$$

by the isomorphism: 


$$
\bigoplus_{0 \leq i<m} L^{i} \cong\left(\bigoplus_{i \geq 0} L^{i} t^{i}\right) / \operatorname{Im}\left(t^{m}-s\right)
$$

Then $\oplus_{0 \leq i<m} L^{i} \cong \pi_{*} \mathcal{O}_{X^{\prime}}$ has a natural regular singular connection induced by that on the smooth part of $X^{\prime}$ (or on a desingularization of $X^{\prime}$ ). Let $\tilde{X}^{\prime}$ be the normalization of $X^{\prime}, \tilde{\pi}: \tilde{X}^{\prime} \rightarrow X$ the natural morphism, and $U$ the intersection of the smooth part of $X$ and $X \backslash s^{-1}(0)$. Then $\left.L^{i}\right|_{U}(0 \leq i<m)$ are the eigenspaces of the transformation group (hence the decomposition is compatible with the connection) and $\pi_{*} \mathcal{O}_{\tilde{X}^{\prime}}$ is Deligne's canonical extension of $\left.\oplus_{0 \leq i<m} L^{i}\right|_{U}$, cf. [11]. In fact, the last fact is well-known to specialists (and easy to check), if $X$ is smooth and $s^{-1}(0)$ is a normal crossing divisor, and we can reduce to this case by Hironaka's desingularization of $(X, U)$. (We can also use the functions of strict Nilsson class.)

4) For $X=\mathbb{P}^{n}$ and $(M, F, K ; W) \in \operatorname{MHW}(X)^{p}$, we have

$$
H^{j}\left(X, \mathrm{Gr}_{F}^{p} M \otimes\left(\Omega_{X}^{n}\right)^{-1}\right)=0 \quad \text { for } j>0 .
$$

This holds also, if each $\Omega_{X}^{i}$ has a filtration whose graduations are negative line bundles for $i>0$ (and if $X$ is smooth projective).

\section{§3. Mixed Hodge Modules of Normal Crossing Type \\ (3.a) Constant Case}

3.1. Let $X$ be a polydisc $\Delta^{n}$ with coordinates $\left(x_{1}, \ldots, x_{n}\right)$. Put $D_{i}=\left\{x_{i}=0\right\}, D_{I}$ $=\cap_{i \in I} D_{i}$. Let $\operatorname{Perv}\left(\mathbb{C}_{X}\right)_{n c}$ be the category of perverse sheaves on $X$ whose characteristic varieties are contained in the union of the conormal bundles of $D_{I}$. Put :

$$
\bar{n}=\{1, \cdots, n\}, \bar{v}=\left\{i \in \bar{n}: v_{i} \neq 0\right\} \quad \text { fir } v \in(\mathbf{C} / \mathbf{Z})^{n} .
$$

We define an abelian category $\mathbf{P}(n)$ as follows:

The objects of $\mathbf{P}(n)$ are finite dimensional $\mathbf{C}$-vector spaces $E_{I}^{v}$ indexed by $\nu \in(\mathbf{C} / \mathbb{Z})^{n}$ and $I \subset \bar{n} \backslash \bar{v}$ such that $E_{I}^{v}=0$ except for a finite number of $(v, I)$, and they are endowed with morphisms:

$$
\begin{aligned}
& \operatorname{can}_{i}: E_{I}^{v} \longrightarrow E_{I \cup\{i\}}^{v} \quad \text { for } i \notin I \cup \bar{v} \\
& \operatorname{Var}_{i}: E_{I}^{v} \longrightarrow E_{I \backslash\{i\}}^{v} \text { for } i \in I \\
& N_{i}: E_{I}^{v} \longrightarrow E_{I}^{v} \text { for any } i
\end{aligned}
$$

satisfying $\operatorname{can}_{i}^{\circ} \operatorname{Var}_{i}=N_{i}(i \in I), \operatorname{Var}_{i}^{\circ} \operatorname{can}_{i}=N_{i}(i \notin I \cup \bar{v})$ and $A_{i} \circ B_{j}=B_{j} \circ A_{i}(i \neq j)$ for $A, B=$ can, Var or $N$ such that $A_{i} \circ B_{j}$ and $B_{j}^{\circ} A_{i}$ are well-defined (e.g. $i \in I$ if $A=$ Var, etc.); in particular the compositions of $\operatorname{can}_{i}, \operatorname{Var}_{j}, \mathrm{~N}_{k}$ are independent of the order if they are well-defined. The morphisms of $\mathbf{P}(n)$ are the morphisms of the vector spaces: $E_{I}^{v} \rightarrow F_{I}^{v}$, compatible with $\operatorname{can}_{i}, \operatorname{Var}_{i}$ and $N_{i}$. 
By inductive use of Deligne's description of the monodromical perverse sheaves (see, for example, [23]) we have an equivalence of categories;

$$
\Psi^{n}: \operatorname{Perv}\left(\mathbb{C}_{X}\right)_{n c} \stackrel{\sim}{\longrightarrow} \mathbb{P}(n)
$$

such that $\left\{E_{I}^{\nu}\right\}=\Psi^{m}(\mathscr{F})$ is defined by

$E_{I}^{v}=\Psi_{x_{1}}^{v_{1}} \cdots \Psi_{x_{n}}^{v_{n}} \mathscr{F} \quad$ for $\mathscr{F} \in \operatorname{Perv}\left(\mathbb{C}_{X}\right)_{n c}$,

$\Psi_{x_{i}}^{v_{i}}=\psi_{x_{i}}^{v_{i}}[-1]$ if $i \notin I$ and $\phi_{x_{i}}^{0}[-1]$ otherwise,

where $\operatorname{can}_{i}, \operatorname{Var}_{i}, \mathrm{~N}_{i}$ are associated to the functors $\psi_{x_{i}}, \phi_{x_{i}, 1}$. Here $\psi_{x_{i}}^{\alpha}$ $=\operatorname{Ker}\left(T_{j, s}-\mathbf{e}(\alpha)\right)($ same for $\phi)$ with $T_{j}=T_{j, s} \circ T_{j, u}$ the Jordan decomposition of the monodromy around $D_{i}$ and $\mathrm{e}(\alpha)=\exp (2 \pi i \alpha)$. In this normal crossing case, we can easily verify that $\Psi^{m}$ is independent of the order of the coordinates.

We define the dual functor $\mathbf{D}$ on $\mathbf{P}(n)$ by:

$$
\begin{aligned}
& \check{E}_{I}^{v}=\mathrm{D}\left(E_{I}^{-v}\right), \check{N}_{i}=-\mathrm{D}\left(N_{i}\right) \\
& \text { căn }_{i}=\mathbb{D}\left(\operatorname{Var}_{i}\right), \operatorname{Văr}_{i}=-\mathrm{D}\left(\operatorname{can}_{i}\right)
\end{aligned}
$$

for $\left\{\check{E}_{I}^{v} ; \operatorname{căn}_{i}, \operatorname{Var}_{i}, \check{N}_{i}\right\}:=\mathbb{D}\left(\left\{E_{I}^{v} ; \operatorname{can}_{i}, \operatorname{Var}_{i}, \mathbf{N}_{i}\right\}\right)$, where $\mathbb{D}$ denotes also the dual functor in the category of finite dimensional vector spaces. Then we have

$$
\Psi^{n} \mathbb{D} \cong \mathbb{D} \Psi^{n}
$$

by $[19,(5.2 .3)]$. Let $\operatorname{Perv}\left(\mathbf{C}_{X}\right)_{n c q u}$ be the full subcategory of $\operatorname{Perv}\left(\mathbb{C}_{X}\right)_{n c}$ consisting of the quasi-unipotent normal crossing perverse sheaves; i.e. we have

$$
\Psi^{n}: \operatorname{Perv}\left(\mathbb{C}_{X}\right)_{n c q u} \stackrel{\sim}{\longrightarrow} \mathbb{P}(n)_{q u},
$$

where $\left\{E_{I}^{v}\right\} \in \mathbb{P}(n)_{q u}$ iff $E_{I}^{v}=0$ for $\nu \notin(\mathbb{Q} / \mathbb{Z})^{n}$.

3.2. With the notation as above, put $\mathscr{D}_{X}=\mathscr{D}_{X, 0}, \mathcal{O}_{X}=\mathcal{O}_{X, 0}$. We define $M\left(\mathscr{D}_{X}\right)_{r n c}$ by the full subcategory of $M\left(\mathscr{D}_{X}\right)$, the category of $\mathscr{D}_{X}$-modules, such that $M \in M\left(\mathscr{D}_{X}\right)_{r n c}$ iff $M$ is generated over $\mathcal{O}_{X}$ by $M^{\alpha}$ for $\alpha \in \mathbb{C}^{n}$ and

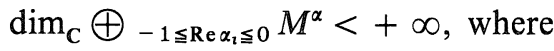

$$
M^{\alpha}=\cap_{i}\left(\cup_{j} \operatorname{Ker}\left(\left(x_{i} \partial_{i}-\alpha_{i}\right)^{j}: M \rightarrow M\right)\right) .
$$

If $M \in M\left(\mathscr{D}_{X}\right)_{\text {rnc }}$, we have

$$
\bigoplus M^{\alpha} \subset M \subset \prod M^{\alpha} .
$$

Note that $M \in M\left(\mathscr{D}_{X}\right)_{r n c}$ iff $M$ is regular holonomic and its characteristic variety has normal crossings as in 3.1. If we choose a subset $\Lambda$ of $\mathbb{C}$ such that the composition: $\Lambda \rightarrow \mathbb{C} \rightarrow \mathbb{C} / \mathbb{Z}$ is bijective, and $-1 \in \Lambda$, we have an equivalence of categories:

$$
\Psi_{\mathscr{D}}^{n}: M\left(\mathscr{D}_{X}\right)_{r n c} \stackrel{\sim}{\longrightarrow} \mathbb{P}(n)
$$


such that $\left\{E_{I}^{v}\right\}=\Psi_{\mathscr{D}}^{m}(M)$ is defined by

$$
E_{I}^{v}=M^{\alpha+1_{I}} \text { for } \alpha \in \Lambda \text { such that } v \equiv \alpha\left(\bmod \mathbf{Z}^{n}\right)
$$

where $\operatorname{can}_{i}, \operatorname{Var}_{i}, N_{i}$ are given by $\partial_{i}, x_{i}, x_{i} \partial_{i}-\alpha_{i}$, and $\left(1_{I}\right)_{j}=1$ if $j \in I$ and 0 otherwise. Here the inverse functor is given by using the inclusion (3.2.1), where $M^{\alpha}$ for $\alpha \notin(\Lambda \cup\{0\})^{n}$ is determined by

$$
\begin{aligned}
& x_{i}: M^{\alpha} \stackrel{\sim}{\longrightarrow} M^{\alpha-1_{i}} \text { if } \alpha_{i} \in \Lambda-\mathbf{N} \\
& \partial_{i}: M^{\alpha} \stackrel{\sim}{\longrightarrow} M^{\alpha+1_{\imath}} \text { if } \alpha_{i} \in(\Lambda \cup\{0\} \backslash\{-1\})+\mathbf{N} .
\end{aligned}
$$

Because Perv $\left(\mathbf{C}_{X}\right)_{n c}$ is independent of the radius of $\Delta$, we have the natural functor $\mathrm{DR}: M\left(\mathscr{D}_{X}\right)_{r n c} \rightarrow \operatorname{Perv}\left(\mathbf{C}_{X}\right)_{n c}$ such that $\Psi_{\mathscr{D}}^{n}=\Psi^{n} \circ \mathrm{DR}$. Then we have $\Psi_{(\mathscr{D})}^{n} \circ \mathrm{D}$ $=\mathbf{D} \circ \Psi_{(\mathscr{D})}^{n}, \quad \mathrm{DR} \circ \mathbf{D}=\mathbf{D} \circ \mathrm{DR}$ by an argument similar to the proof of $[19$, 3.4.2]. Let $M\left(\mathscr{D}_{X}\right)_{r n c q u}$ be the full subcategory of $M\left(\mathscr{D}_{X}\right)_{r n c}$ such that $M \in M\left(\mathscr{D}_{X}\right)_{r n c q u}$ iff $M^{\alpha}=0$ for $\alpha \notin \mathbf{Q}^{n}$ (i.e. $\left.\Psi_{\mathscr{D}}^{n}(M) \in \mathbf{P}(n)_{q u}\right)$. In this case, the above assertion follows from [loc. cit], because the filtration $V^{(i)}$ along $D_{i}$ is given by $V_{\beta}^{(i)}=M \cap \prod_{\alpha_{1} \leq \beta} M^{\alpha}$.

From now on we restrict to the quasi-unipotent case.

3.3. Theorem. For $\mathscr{F} \in \operatorname{Perv}\left(\mathbb{C}_{X}\right)_{n c q u}, m \in \mathbf{N}^{n}(m \neq 0)$ and $g=x^{m}\left(:=\prod x_{i}^{m_{1}}\right)$, set $\left\{E_{I}^{v} ; \operatorname{can}_{i}, \quad \operatorname{Var}_{i}, \quad N_{i}\right\}=\psi^{n}(\mathscr{F}), \quad \bar{m}=\left\{i \in \bar{n}: m_{i} \neq 0\right\}, \quad E_{I}^{v}[N]=E_{I}^{v} \otimes \mathbf{C}[N], \quad\left(N_{*}\right.$ $\left.-m_{*} N\right)_{J}=\prod_{i \in J}\left(N_{i}-m_{i} \mathrm{~N}\right), N_{J}=\prod_{i \in J} N_{i}\left(\right.$ same for $\left.\operatorname{can}_{J}, \operatorname{Var}_{J}\right)$ and define $\left\{\widetilde{E}_{I}^{v}\right.$; can $\left._{i}, \operatorname{Var}_{i}, \tilde{N}_{i}\right\} \in \mathbf{P}(n+1)_{q u}$ as follows:

$$
\tilde{E}_{I}^{v}= \begin{cases}\operatorname{Coker}\left(N_{*}-m_{*} N\right)_{I \cap \bar{m}} & \text { if } 0 \notin I \\
\operatorname{Coker}\left(\begin{array}{c}
\left(\left(N_{*}-m_{*} N\right)_{I \cap \bar{m}}-N_{I \cap \bar{m}}\right) N^{-1},-\operatorname{Var}_{I \cap \bar{m}} \\
\operatorname{can}_{I \cap \bar{m}}
\end{array}\right) \text { if } 0 \in I\end{cases}
$$

for $v=\left(v_{0}, \cdots, v_{n}\right) \in \mathbf{N}^{n+1}, I \subset \bar{n} \cup\{0\} \backslash \bar{v}$, where the above morphisms are (injective) endomorphisms of

$$
E_{I \backslash \bar{m}}^{v^{\prime}+v_{0} m}[N] \text { and } E_{I^{\prime} \backslash \bar{m}}^{v^{\prime}}[N] \oplus E_{I^{\prime}}^{v^{\prime}}[N]
$$

respectively, with $v^{\prime}=\left(v_{1}, \cdots, v_{n}\right)$ and $I^{\prime}=I \backslash\{0\}$. The morphisms can ${ }_{i}$, etc. are defined by the morphisms of mapping cones:

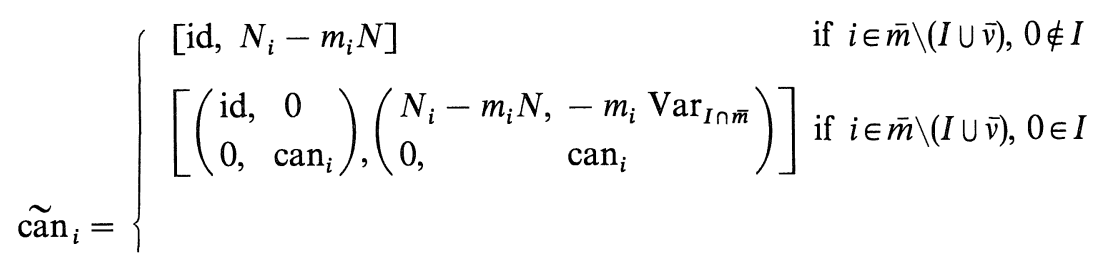




$$
\begin{aligned}
& \mid \begin{array}{ll}
{\left[\operatorname{can}_{i}, \operatorname{can}_{i}\right]} & \text { if } i \in \bar{n} \backslash(\bar{m} \cup \bar{v} \cup I) \\
{\left[\begin{array}{l}
N \\
\left.\left.-\operatorname{can}_{I \cap \bar{m}}\right),\left(\begin{array}{l}
\text { id } \\
0
\end{array}\right)\right]
\end{array}\right.} & \text { if } i=0,
\end{array}
\end{aligned}
$$

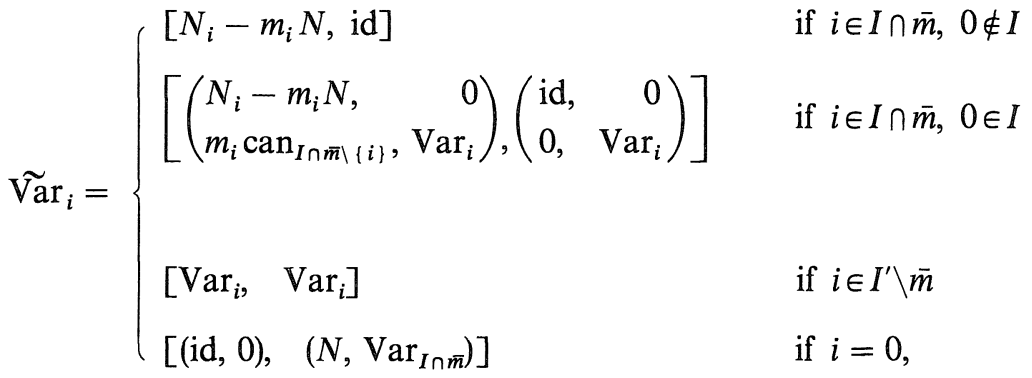

$$
\begin{aligned}
& \widetilde{N}_{i}=\left\{\begin{array}{lr}
{\left[N_{i}-m_{i} N, N_{i}-m_{i} N\right]} & \text { if } i \neq 0 \\
{[N, N]} & \text { if } i=0 .
\end{array}\right.
\end{aligned}
$$

Then $\Psi_{g} \mathscr{F} \in \operatorname{Perv}\left(\mathbb{C}_{X \times S}\right)_{n c q u}$, and we have a functorial isomorphism in $\mathbb{P}(n+1)_{q u}$ :

$$
\Psi^{n+1}\left(\Psi_{g} \mathscr{F}\right) \cong\left\{\widetilde{E}_{I}^{v} ; \tilde{\operatorname{can}}_{i},{\widetilde{\operatorname{Var}_{i}}}_{i} \tilde{N}_{i}\right\},
$$

where $S=\Delta$ the open disc and $\Psi_{g} \mathscr{F}$ is Deligne's nearby cycle functor (i.e. Verdier's specialization in the codimension one case, cf. [23]); in particular, $\Psi^{n}\left(\psi_{g}^{\alpha} \mathscr{F}\right) \cong\left\{\widetilde{E}_{I}^{v}: I \not \nexists 0, v_{0} \equiv \alpha\right\}, \Psi^{n}\left(\phi_{g}^{\alpha} \mathscr{F}\right) \cong\left\{\widetilde{E}_{I}^{v}: I \ni 0\right\}$ in $\mathbb{P}(n)$.

By 3.1.2, the assertion is reduced to the following

3.4. Theorem. For $M \in M\left(\mathscr{D}_{X}\right)_{r n c q u}$ and $g=x^{m}$ as above, $\tilde{M}:=\left(i_{g}\right)_{*} M\left(\cong M\left[\partial_{t}\right]\right)$ has the filtration $V$ along $X \times\{0\}$ indexed by $\mathbb{Q}$ such that $V_{\alpha} \tilde{M}$ is generated over $\mathscr{D}_{x}$ by

$$
\begin{array}{ll}
M^{v} \otimes 1 \text { with } m_{i} \alpha \geq v_{i} & \text { if } \alpha<0 \\
M^{v} \otimes \partial_{t}^{j} \text { with } m_{i}(\alpha-j) \geq v_{i} & \text { in general }
\end{array}
$$

where $v \in \mathbb{Q}^{n}$ and $j \in \mathbf{N}$. For $\left\{E_{I}^{v} ; \operatorname{can}_{i}, \operatorname{Var}_{i}, N_{i}\right\}=\Psi_{\mathscr{D}}^{n}(M)$ and $\left\{\widetilde{E}_{I}^{v} ; \tilde{\operatorname{can}}_{i}, \widetilde{\operatorname{Var}}_{i}\right.$, $\left.\tilde{N}_{i}\right\} \in \mathbb{P}(n+1)_{q u}$ as defined in 3.3, we have functorial isomorphisms:

$$
\begin{gathered}
\operatorname{DR}\left(\Psi_{g} M\right) \cong \Psi_{g} \operatorname{DR}(M) \text { in } \operatorname{Perv}\left(\mathbb{C}_{X \times S}\right)_{n c q u} \\
\Psi_{\mathscr{D}}^{n+1}\left(\Psi_{g} M\right) \cong\left\{\widetilde{E}_{I}^{v} ; \tilde{\operatorname{can}}_{i}, \widetilde{\operatorname{Var}}_{i}, \tilde{N}_{i}\right\} \text { in } \mathbb{P}(n+1)_{q u}
\end{gathered}
$$

where $\Psi_{g} M:=\mathrm{Gr}^{V} \tilde{M} \otimes \mathrm{Gr}_{\mathscr{D}} \mathscr{D}_{X \times S}$.

Proof. We denote by $V$ the filtration on $\tilde{M}$ defined as above. The action of 
$\mathscr{D}_{X \times S}$ on $\tilde{M}=M\left[\partial_{t}\right]$ is given by:

$$
\begin{aligned}
& \left(u \otimes \partial_{t}^{j}\right) a=u a \otimes \partial_{t}^{j},\left(u \otimes \partial_{t}^{j}\right) \partial_{i}=u \partial_{i} \otimes \partial_{t}^{j}-u\left(\partial_{i} g\right) \otimes \partial_{t}^{j+1} \\
& \left(u \otimes \partial_{t}^{j}\right) t=u g \otimes \partial_{t}^{j}+j u \otimes \partial_{t}^{j-1},\left(u \otimes \partial_{t}^{j}\right) \partial_{t}=u \otimes \partial_{t}^{j+1}
\end{aligned}
$$

for $a \in \mathcal{O}_{X}, u \in M$, where $t$ is the coordinate of $S \subset \mathbf{C}$ and we use the coordinates $\left(t-g, x_{1}, \cdots, x_{n}\right)$ of $X \times S$ to get the isomorphism $\tilde{M} \cong M\left[\partial_{t}\right]$. In particular, we have

$$
\left(u \otimes \partial_{t}^{j}\right) x_{i} \partial_{i}=\left(u \otimes \partial_{t}^{j}\right)\left(N_{i}+v_{i}-m_{i}(s-j)\right) \text { for } u \in M^{v},
$$

where $s=t \partial_{t}$ and $\left(u \otimes \partial_{t}^{j}\right) N_{i}=u\left(x_{i} \partial_{i}-v_{i}\right) \otimes \partial_{t}^{j}$ if $u \in M^{v}$. This implies that $s$ $-\alpha$ is nilpotent on $\operatorname{Gr}_{\alpha}^{V} \tilde{M}$ and $V_{\alpha} \tilde{M}$ are $V_{0} \mathscr{D}_{X \times S^{-}}$-sub-Modules. Thus we get the first assertion, because $\left(V_{\alpha} \tilde{M}\right) t=V_{\alpha-1} \tilde{M}(\alpha<0)$ and the coherence of $V_{\alpha} \tilde{M}$ follow from (3.2.3) and $M^{v} g=M^{v-m}$ for $v \in \mathbf{Q}^{n}$ such that $v_{i}<0$ for $i \in \bar{m}$. We define the morphisms

$$
A_{I}^{v}: \widetilde{E}_{I}^{v} \longrightarrow\left(\mathrm{Gr}_{v_{0}+\left(1_{I}\right)_{0}}^{V} \tilde{M}\right)_{I^{\prime}}^{v^{\prime}} \subset\left(\Psi_{g} M\right)_{I}^{v}
$$

for $v \in(\mathbb{Q} \cap[-1,0))^{n+1}, I \subset \bar{n} \cup\{0\} \backslash \bar{v}$ (where $\bar{v}=\left\{i \in \bar{n} \cup\{0\}: v_{i} \neq-1\right\}$ ) by

$$
\begin{aligned}
& A_{I}^{v}(u)=u x^{k(v)} x_{I \cap \bar{m}}^{-1} \otimes 1 \text { if } 0 \notin I \\
& A_{I}^{v}(u, v)=u x^{k(v)} x_{I \cap \bar{m}}^{-1} \otimes \partial_{t}+v \otimes 1 \text { if } 0 \in I,
\end{aligned}
$$

for $u \in E_{I \backslash \bar{m}}^{\mu},(u, v) \in\left(E_{I^{\prime} \backslash \bar{m}}^{v^{\prime}}, E_{I^{\prime}}^{v^{\prime}}\right)$, where $E_{J}^{\mu}$ is identified with $M_{J}^{\mu}:=M^{\mu+1_{J}} \subset M$, and $\mu \in(\mathbb{Q} \cap[-1,0))^{n}, k(v) \in \mathbf{N}^{n}+1_{\bar{m} \backslash \bar{v}}$ are defined by

$$
\mu=v^{\prime} \quad v_{0} m+k(v) \text {. }
$$

In particular, $\mu=v^{\prime}$ and $k(v)=m$ (i.e. $\left.u x^{k(v)} x_{I \cap \bar{m}}^{-1}=u g x_{I \cap \bar{m}}^{-1}\right)$ if $0 \in I$. Here the action of $N$ on $\operatorname{Gr}_{\alpha}^{V} \tilde{M}$ is induced by $s-\alpha$, and $x_{J}:=\prod_{j \in J} x_{j}$. In fact, (3.4.7) implies

$$
\mu_{i}+\left(1_{I \backslash \bar{m}}\right)_{i}-k(v)_{i}+\left(1_{I \cap \bar{m}}\right)_{i}-m_{i} v_{0}=v_{i}+\left(1_{I}\right)_{i} \leq 0
$$

(where the equality holds iff $i \in I$ ), i.e. $\operatorname{Im} A_{I}^{v} \subset V_{v_{0}+\left(1_{I}\right)_{0}} \tilde{M}$. Then $\operatorname{Im} A_{I}^{v}$ $\subset\left(\Psi_{g} M\right)_{I}^{v}$ and the well-definedness of $A_{I}^{v}$ (i.e. (3.4.6) induces $\mathbb{C}[N]$-linear morphisms from the cokernels in (3.3.1)) follow from:

$$
\begin{aligned}
& \left(u x^{k(v)} x_{I \cap \bar{m}}^{-1} \otimes 1\right)\left(x_{i} \partial_{i}-v_{i}-\left(1_{I}\right)_{i}\right)=\left(u x^{k(v)} x_{I \cap \bar{m}}^{-1} \otimes 1\right)\left(N_{i}-m_{i}\left(s-v_{0}\right)\right) \\
& (v \otimes 1)\left(x_{i} \partial_{i}-v_{i}-\left(1_{I}\right)_{i}\right)=(v \otimes 1)\left(N_{i}-m_{i} s\right) \\
& \left(u x^{k(v)} x_{I \cap \bar{m}}^{-1} \otimes 1\right)\left(N_{*}-m_{*}\left(s-v_{0}\right)\right)_{I \cap \bar{m}}=\left(u x^{k(v)} \otimes 1\right) \partial_{I \cap \bar{m}} \\
& \left(u \partial_{I \cap \bar{m}} \otimes 1\right)+\left(u g x_{I \cap \bar{m}}^{-1} \otimes \partial_{t}\right)\left(\left(N_{*}-m_{*} s\right)_{I \cap \bar{m}}-N_{I \cap \bar{m})} s^{-1}=(u \otimes 1) \partial_{I \cap \bar{m}}\right. \\
& (v \otimes 1) s=\left(\left(v x_{I \cap \bar{m}}\right) g x_{I \cap \bar{m}}^{-1} \otimes \partial_{t}\right) .
\end{aligned}
$$


(Here, we use $\left[N_{j}, x^{k}\right]=\left[N_{j}, s\right]=0$ and $v_{i}=-1$ for $i \in I$.) In particular, we get $\Psi_{g} M \in M\left(\mathscr{D}_{X \times S}\right)_{r n c q u}$ by $\operatorname{Im} A_{I}^{v} \subset\left(\Psi_{g} M\right)_{I}^{v}$ and the first assertion. We can verify similarly that the morphisms $\partial_{i}, x_{i}$ and $x_{i} \partial_{i}-v_{i}$ on $\operatorname{Im} A_{I}^{v}$ are expressed by the second term in the definition of $\tilde{c a n}_{i}, \widetilde{\operatorname{Var}}_{i}$ and $\tilde{N}_{i}$ in 3.3, where $x_{0}=t$. Here note that $\widetilde{E}_{I}^{v}=0$ (and $\left.\left(\Psi_{g} M\right)_{I}^{v}=0\right)$ if $I \cap \bar{m}=\phi$. Because the morphisms in (3.3.1) are injective, the first term of $\tilde{c a n}_{i}$, etc. are uniquely determined by the commutativity (and the first term), and the functors $\left\{E_{I}^{\nu}\right\} \mapsto\left\{\widetilde{E}_{I}^{\nu}\right\}$ are exact. Therefore the assertion is reduced to the case $M$ simple using a filtration on $M$, and follows from the next propositon, because the assertion is clear by $[19,3.2 .6]$ if supp $M \subset g^{-1}(0)$, and we may assume $\operatorname{supp} M=(X, 0)$ by $[19$, 3.2.4].

3.5. Proposition. With the notation as in 3.4 , assume $M$ is simple, i.e. $M_{J}^{\mu} \cong \mathbb{C}$ for one $(\mu, J)$ and 0 otherwise $(M$ will be denoted by $M(\mu, J))$, and $\operatorname{supp} M=(X$, $0)$, i.e. $J=\phi$. Then we have a free resolution of $M$ by a Koszul complex:

$$
K=K\left(\mathscr{D}_{X} ;\left(x_{i} \partial_{i}-\mu_{i}\right) \cdot(i \in \bar{\mu}), \partial_{i} \cdot(i \notin \bar{\mu})\right)[n]
$$

where $\left(x_{i} \partial_{i}-\mu_{i}\right) \cdot$ and $\partial_{i} \cdot$ are left multiplications. Let $F$ be a filtration on $K, M$ such that

$$
F_{p} K^{-j}=\left(F_{p-j} \mathscr{D}_{X}\right)^{m_{j}}, F_{p}=\left(M_{J}^{\mu}\right) F_{p} \mathscr{D}_{X}
$$

where $m_{j}=n ! / j !(n-j) ! . \quad$ Then $(K, F) \rightarrow(M, F)$ is a filtered quasi-isomorphism so that $(M, F)$ is Cohen-Macaulay, $\mathbb{D}(M(\mu, \phi), F) \cong(M(-\mu, \phi), F[-n])$ (where $\mu$, $\left.-\mu \in(\mathbb{Q} / \mathbb{Z})^{n}\right),(M, F)$ is quasi-unipotent and regular along $g[19,3.2 .1]$ and the morphisms $A_{I}^{v}$ in the proof of 3.4 are isomorphisms. Furthermore, put $I(\alpha)$ $=\left\{i \in \bar{m}: \mu_{i}-k^{\prime}(\alpha)_{i}=m_{i} \alpha\right\}$ where $k^{\prime}(\alpha) \in \mathbf{N}^{\bar{m}}$ satisfies:

$$
\mu_{i}-k^{\prime}(\alpha)_{i} \leq m_{i} \alpha<\mu_{i}-k^{\prime}(\alpha)_{i}+1 \text {, }
$$

then:

$$
\begin{aligned}
& F_{p} V_{\alpha} \tilde{M}=\left(M_{J}^{\mu} x^{k^{\prime}(\alpha)} \otimes 1\right) F_{p}\left(\mathscr{D}_{X}[s]\right) \quad \text { for } \alpha<0, \\
& \left(F_{p} \operatorname{Gr}_{\alpha}^{V} \tilde{M}\right) \partial_{t}=F_{p+1} \operatorname{Gr}_{\alpha+1}^{V} \tilde{M} \text { for } \alpha \geq-1, \\
& N^{i}: \operatorname{Gr}_{i}^{W} \operatorname{Gr}_{\alpha}^{V}(\tilde{M}, F) \stackrel{\sim}{\longrightarrow} \operatorname{Gr}_{-i}^{W} \operatorname{Gr}_{\alpha}^{V}(\tilde{M}, F[-i]) \text { for } i>0, \\
& \left.P \operatorname{Gr}_{i}^{W} \operatorname{Gr}_{\alpha}^{V}(\tilde{M}, F)=\underset{I \subset I(\alpha),|I|=i+1}{\bigoplus}\left(M\left(\mu-\left(k^{\prime}(\alpha)+1_{I(\alpha)}\right)-m \alpha\right), I \cup J\right), F\right) \\
& \text { for } \alpha<0, i \geq 0, \\
& \partial_{t}: \operatorname{PGr}_{i+1}^{W} \operatorname{Gr}_{-1}^{V}(\tilde{M}, F) \stackrel{\sim}{\longrightarrow} P_{G_{i}^{W}} \operatorname{Gr}_{0}^{V}(\tilde{M}, F[-1]) \text { for } i \geq 0,
\end{aligned}
$$

where $F_{p}\left(\mathscr{D}_{X}[s]\right)=\sum F_{p-i} \mathscr{D}_{X} s^{i}, W$ is the monodromy filtration on $\mathrm{Gr}_{\alpha}^{V} \tilde{M}$ and $P$ is the primitive part. 
Proof. The first assertion is clear, because $\mathscr{H}^{i} \mathrm{Gr}^{F} K=0$ for $i \neq 0$ (hence $(K, F)$ is strict) and $\mathscr{H}^{0}(K, F) \cong(M, F)$ by definition. Then we may assume $\bar{m}=\bar{n}$, because the vanishing cycle functors are compatible with external products, i.e. $\left(\Psi_{g}\left(M_{1}, F\right)\right) \bigotimes\left(M_{2}, F\right)=\Psi_{g}\left(\left(M_{1}, F\right) \bigotimes\left(M_{2}, F\right)\right) . \quad($ Note that $(M, F)$ and $(K, F)$ are the external products of objects on $\Delta$.) Then it is enough to show (3.5.1-2) and the filtered isomorphism for $\alpha<0$ :

$$
\underset{0 \leq i<|I|}{\bigoplus}\left(\left(M_{J}^{\mu} x^{k^{\prime}(\alpha)} x_{I(\alpha) \backslash I} \otimes 1\right)(s-\alpha)^{i}, F\right) \stackrel{\sim}{\longrightarrow}\left(\operatorname{Gr}_{\alpha}^{V}(M, F)\right)_{I}^{v}
$$

for $v, I$ such that $I \subset I(\alpha), v=\mu-\left(k^{\prime}(\alpha)+1_{I(\alpha)}\right)-m \alpha$, where $F$ is by the order of $s-\alpha$ on the left hand side. In fact, (3.5.5) and (3.5.3) for $\alpha \geq 0$ follow from (3.5.2) and (3.5.3) for $\alpha<0$, because (3.5.3) implies the strictness of

$$
N:\left(\operatorname{Gr}_{\alpha}^{V}(M, F), W\right) \longrightarrow\left(\operatorname{Gr}_{\alpha}^{V}(M, F[-1], W[2])\right.
$$

by Lefschetz decomposition and [7, (1.6.5)], and (3.5.2) and [19, (5.1.12)] imply $\left(\operatorname{Gr}_{0}^{V}(M, F), W\right) \cong \operatorname{Im}\left(N:\left(\operatorname{Gr}_{-1}^{V}(M, F[1]), W[-1]\right) \rightarrow\left(\operatorname{Gr}_{-1}^{V}(M, F), W[1]\right)\right)$.

By (3.5.1) and $\operatorname{Im} A_{I}^{v} \subset\left(\Psi_{g} \tilde{M}\right)_{I}^{v}$ in the proof of 3.4, we have the compatibility of the filtration $F$ with the decomposition: $\operatorname{Gr}_{\alpha}^{V} \tilde{M} \subset \prod_{\nu}\left(\operatorname{Gr}_{\alpha}^{V} \tilde{M}\right)^{v}$, i.e. $m \in F_{p} \operatorname{Gr}_{\alpha}^{V} \tilde{M}$ iff its component in $\left(\mathrm{Gr}_{\alpha}^{V} \tilde{M}\right)^{v}$ belongs to $F_{p} \operatorname{Gr}_{\alpha}^{V} \tilde{M}$ for any $v \in \mathbb{Q}^{n}$ (because the filtration $F$ of $\operatorname{Gr}_{\alpha}^{V} \tilde{M}$ is generated by $\operatorname{Gr}_{\alpha}^{V}\left(M_{J}^{\mu} x^{k^{\prime}(\alpha)} \otimes 1\right)$ over $\left(\mathscr{D}_{X}[s]\right.$, $F)$ ); therefore the right hand side of (3.5.6) has a meaning. Moreover, by (3.5.1), we have for $\alpha<0, v \in \mathbb{Q}^{n}$ :

$$
\begin{aligned}
& x_{i}:\left(\operatorname{Gr}_{\alpha}^{V}(M, F)\right)^{v} \stackrel{\sim}{\longrightarrow}\left(\operatorname{Gr}_{\alpha}^{V}(M, F)\right)^{v-1_{i}} \\
& \partial_{i}:\left(\operatorname{Gr}_{\alpha}^{V}(M, F)\right)^{v} \stackrel{\text { if } v_{i}<0}{\longrightarrow}\left(\operatorname{Gr}_{\alpha}^{V}(M, F[-1])\right) v+1_{i} \text { if } v_{i}>-1 .
\end{aligned}
$$

Therefore (3.5.3) for $\alpha<0$ and (3.5.4) follow from (3.5.6), because $\left(\operatorname{Gr}_{\alpha}^{V} M\right)_{I}^{v}=0$ for other $v, I$ than in (3.5.6) (in fact, $\operatorname{Im} A_{I}^{v} \subset\left(\Psi_{g} M\right)_{I}^{v}$, cf. the proof of 3.4). Here note that the morphism in (3.5.6) coincides with $A_{I}^{v}$ (where $\alpha, v, k^{\prime}(\alpha), I$ and $\mathrm{I}(\alpha)$ correspond to $v_{0}, v^{\prime}, k(v)-1_{I(\alpha)}, I \cap \bar{m}$ and $\bar{m} \backslash \bar{v}$ respectively), because $N_{i}=0$ on $M_{J}^{\mu}$ and $J=\phi$.

We show (3.5.6) is also reduced to (3.5.1). In fact the strict surjectivity of (3.5.6) follows from (3.5.1) by the above argument, because we have $\tilde{N}_{i}=-m_{i} N$ on $\operatorname{Im} A_{I}^{v}$ by $N_{i}=0$ on $M_{J}^{\mu}$, cf. 3.3-4. Therefore it is enough to show the injectivity for $I=I(\alpha)$, i.e.

$$
\operatorname{Gr}_{\alpha}^{V}\left(M_{J}^{\mu} x^{k^{\prime}(\alpha)} \otimes 1\right)(s-\alpha)^{p} \neq 0 \text { in } \operatorname{Gr}_{\alpha}^{V} \tilde{M}
$$

for $p<|I(\alpha)|$, because $\tilde{N}_{i}=-m_{i} N, M_{J}^{\mu} \cong \mathbf{C}$ and $N=s-\alpha$ is nilpotent. Taking $\mathrm{Gr}_{p}^{F}$, it is sufficient to show 


$$
\operatorname{Gr}_{p}^{F}\left(\left(M_{J}^{\mu} x^{k^{\prime}(\alpha)} \otimes 1\right)(s-\alpha)^{p}\right) \propto \operatorname{Gr}_{p}^{F} V_{<\alpha} \tilde{M}
$$

Let $G$ be the filtration of $\operatorname{Gr}_{P}^{F} \tilde{M}=\oplus \mathrm{Gr}_{p-i}^{F} M \otimes \partial_{t}^{i}$ by the order of $\partial_{t}$, then

$$
\begin{aligned}
& \operatorname{Gr}_{p}^{G} \operatorname{Gr}_{p}^{F}\left(\left(M_{J}^{\mu} x^{k^{\prime}(\alpha)} \otimes 1\right)(s-\alpha)^{p}\right)=M_{J}^{\mu} x^{k^{\prime}(\alpha)} g^{p} \otimes \partial_{t}^{p} \\
& \operatorname{Gr}_{p}^{G} \operatorname{Gr}_{p}^{F} V_{<\alpha} \tilde{M}=\sum_{l \in \mathbf{N}^{n},|l| \leq p} M_{J}^{\mu} x^{k^{\prime}(\alpha)+1_{I(\alpha)}-l} g^{p} \mathcal{O}_{x} \otimes \partial_{t}^{p},
\end{aligned}
$$

because $\operatorname{Gr}_{p}^{F} V_{<\alpha} \tilde{M}=\left(M_{J}^{\mu} x^{k^{\prime}(\alpha)+1_{I(\alpha)}} \otimes 1\right) \mathrm{Gr}_{p}^{F}\left(\mathscr{D}_{X}[s]\right)$ by (3.5.1). Thus we get (3.5.6).

We now show (3.5.2). Let $u=\sum u_{i} \otimes \partial_{t}^{i} \in F_{p+1} V_{\alpha+1} \tilde{M}$, then $u_{i} \in F_{p+1-i} M$ by definition. We have $u_{0}=u^{\prime} P$ for $u^{\prime} \in M_{J}^{\mu}, \quad P \in F_{p+1} \mathscr{D}_{X}$, and $\left(u^{\prime} \otimes 1\right) P \in F_{p+1} V_{<0} \tilde{M}$ by the first assertion of 3.4. Then $u$ $-\left(u^{\prime} \otimes 1\right) P \in\left(F_{p} V_{\alpha} M\right) \partial_{t}$ by (3.4.3), because $\alpha \geq-1$ and $\partial_{t}: \operatorname{Gr}_{\beta}^{V} \tilde{M} \rightarrow \operatorname{Gr}_{\beta+1}^{V} \tilde{M}$ is injective for $\beta>-1$. Thus we get (3.5.2).

For the proof of (3.5.1) we have to show the strict injectivity of $\left(V_{\alpha} \tilde{M}, F^{\prime}\right)$ $\rightarrow(\tilde{M}, F)$, where the filtration $F^{\prime}$ on $V_{\alpha} \tilde{M}$ is defined by (3.5.1). Let $G$ be the filtration of $\tilde{M}=M\left[\partial_{t}\right]$ by the order of $\partial_{t}$. Then $F_{p} \subset G_{p}$ and it is enough to show the injectivity of $\mathrm{Gr}^{F^{\prime}} V_{\alpha} \tilde{M} \rightarrow \mathrm{Gr}^{G} \tilde{M}$. Consider a filtered Koszul complex :

$$
\left.(\tilde{K}, F)=K\left(\mathscr{D}_{X}[s] ;\left(x_{i} \partial_{i}-\mu_{i}+k^{\prime}(\alpha)_{i}+m_{i} s\right) \cdot\right), F\right)[n]
$$

where $F$ is the filtration by the order of $\partial_{i}, s$ shifted by the degree of complex. Then $\mathscr{H}^{i} \mathrm{Gr}^{F} \tilde{K}=0$ for $i \neq 0$, hence $(\tilde{K}, F)$ is strict, and we have a filtered $\mathscr{D}_{X}[s]$-linear morphism: $\mathscr{H}^{0}(\tilde{K}, F) \rightarrow\left(V_{\alpha} M, F^{\prime}\right)$, if we choose a basis of $M_{J}^{\mu}$. By definition this morphism is strictly surjective, hence it is enough to show the injectivity of

$$
\mathscr{H}^{0} \mathrm{Gr}^{F} \tilde{K} \longrightarrow \mathcal{O}_{X}[\tau]\left(\subset M[\tau]=\mathrm{Gr}^{G} \tilde{M}\right),
$$

where $\tau=\operatorname{gr} \partial_{t}$ and $\operatorname{Gr}^{F} \tilde{K}^{0}=\mathcal{O}_{X}[\xi, s] \rightarrow \mathcal{O}_{X}[\tau]$ is defined by

$$
\xi_{i} \longmapsto-\left(\partial_{i} g\right) \tau, s \longmapsto g \tau .
$$

Let $\mathcal{O}_{X}(* D)$ and $\operatorname{Gr}^{F} \tilde{K}(* D)$ be the localization of $\mathcal{O}_{X}$ and $\operatorname{Gr}^{F} \tilde{K}$ by $g$. Then the localization of the above morphism by $g$ is injective. Therefore it is sufficient to show the injectivity of $\mathscr{H}^{0} \mathrm{Gr}^{F} \widetilde{K} \rightarrow \mathscr{H}^{0}\left(\mathrm{Gr}^{F} \tilde{K}(* D)\right)$; or equivalently, $\mathscr{H}^{i}\left(\mathrm{Gr}^{F} \widetilde{K}(* D) / \mathrm{Gr}^{F} \widetilde{K}\right)=0(i \neq 0)$, i.e. $\left\{x_{i} \xi_{i}+m_{i} s\right\}$ is a regular sequence of $\left(\mathcal{O}_{X}(* D) / \mathcal{O}_{X}\right)[\xi, s]$. But $\left\{x_{i} \xi_{i}+m_{i} s, g\right\}$ is a regular sequence of $\mathcal{O}_{X}[\xi, s]$, because $\operatorname{codim}\left\{x_{i} \xi_{i}+m_{i} s=0, g=0\right\}=n+1$ in $\operatorname{Spec} \mathcal{O}_{X}[\xi, s]$. Therefore $\left\{x_{i} \xi_{i}+m_{i} s\right\}$ is a regular sequence of $\left(\mathcal{O}_{X} / \mathcal{O}_{X} g\right)[\xi, s]$ and $\left(\mathcal{O}_{X}(* D) / \mathcal{O}_{X}\right)[\xi, s]$. This completes the proof of 3.5 and $3.3-4$.

As a corollary of 3.3 , we get;

3.6. Proposition. Let $\mathscr{F}, g, E_{I}^{v}$ and $\widetilde{E}_{I}^{v}$ be as in 3.3, and $W$ a finite increasing filtration of $\mathscr{F}$ such that 


$$
\begin{array}{ll}
\widetilde{c a n}_{i}\left(W_{j} E_{I}^{v}\right) \subset W_{j-1} E_{I \cup\{i\}}^{v} & \text { for } i \notin I \cup \bar{v} \\
\widetilde{\operatorname{Var}}_{i}\left(W_{j} E_{I}^{v}\right) \subset W_{j-1} E_{I \backslash i \uparrow\}}^{v} & \text { for } i \in I \\
N_{i}\left(W_{J} E_{I}^{v}\right) \subset W_{j-2} E_{I}^{v} & \text { for any } i,
\end{array}
$$

where $W$ denotes also the corresponding filtration on $\left\{E_{I}^{\nu}\right\}=\Psi^{n}(\mathscr{F})$. Then the relative monodromy filtration $W$ of $\left(\Psi_{g}(\mathscr{F}, W), N\right)$ exists and $W_{i} \widetilde{E}_{I}^{v}$ are expressed as the mapping cone of

$$
\begin{aligned}
& W_{i+r+1}\left(E_{I \backslash m}^{v^{\prime}+v_{0} m}[N]\right) \rightarrow W_{i-r+1}\left(E_{I \backslash \bar{m}}^{v^{\prime}+v_{0} m}[N]\right) \\
& W_{i+r}\left(E_{I^{\prime} \backslash \bar{m}}^{v^{\prime}}[N]\right) \oplus W_{i+2}\left(E_{I^{\prime}}^{v^{\prime}}[N]\right) \rightarrow W_{i-r+2}\left(E_{I^{\prime} \backslash \bar{m}}^{v^{\prime}}[N]\right) \oplus W_{i}\left(E_{I^{\prime}}^{v^{\prime}}[N]\right),
\end{aligned}
$$

where $r=|I \cap \bar{m}|, W_{i}\left(E_{I}^{v}[N]\right)=\sum W_{i+2 j} E_{I}^{v} \otimes N^{j}$ and the morphisms are defined as in (3.3.1).

Proof. It is enough to show that the filtration $W$ defined in the assertion is the relative monodromy filtration of $\widetilde{E}_{I}^{v}$ for each $v, I$, because the relative monodromy filtration is functorial. Let $L$ be the filtration of $E_{I}^{v}[N]$ defined by

$$
L_{i}\left(E_{I}^{v}[N]\right)=\left(L_{i} E_{I}^{v}\right)[N]
$$

where $\left\{L_{i} E_{I}^{\nu}\right\}=\Psi^{n}\left(W_{i} \mathscr{F}\right)$. Then it induces the filtration $L$ on the mapping cone of (3.3.1), which gives the filtration $L=\Psi_{g} W$ on $\left\{\widetilde{E}_{I}^{v}\right\}=\Psi^{n+1} \Psi_{g} \mathscr{F}$. We see that $\mathrm{Gr}^{W} \mathrm{Gr}^{L}$ of (3.3.1) are injective and $\mathrm{Gr}^{W} \mathrm{Gr}^{L}$ of the mapping cone of (3.3.1) calculates $\mathrm{Gr}^{W} \mathrm{Gr}^{L} \widetilde{E}_{I}^{v}$. Then the assertion is clear, because $\mathrm{Gr}^{L}$ of (3.3.1) is $N^{r}$ and $\left(N^{r-1}, N\right)$, and $W_{i} \mathrm{Gr}_{k}^{L}\left(E_{I}^{v}[N]\right)=\left(\mathrm{Gr}_{k}^{L} E_{I}^{v}\right)[N] N^{j}$ with $k-i \leq 2 j \leq k-i+1$.

3.7. With the notation of 3.1-2, let $\operatorname{MFW}\left(\mathscr{D}_{X}\right)_{g s n c}$ be the category whose objects are $(M ; F, W)$ where $(M, F)$ is a filtered $\mathscr{D}_{X}$-module such that $M \in M\left(\mathscr{D}_{\mathrm{X}}\right)_{r n c q u}$ (cf. 3.3) and $W$ is a finite increasion filtration satisfying

(3.7.1) $\operatorname{Gr}_{k}^{W}(M, F)$ are isomorphic to direct sums of filtered $\mathscr{D}_{X}$-modules of type $(M(\mu, J), F[q])$ in 3.5 such that $k=|J|-n-2 q$.

(3.7.2) $n+2$ filtrations $F, W, V^{(i)}(1 \leq i \leq n)$ on $M$ are compatible [19, 1.1.13],

where $V^{(i)}$ is the filtration $V$ along $D_{i}$. Note that (3.7.1-2) are independent of the coordinates such that $x_{i}^{-1}(0)=D_{i}$. In fact (3.7.1) is equivalent to

(3.7.3) $\operatorname{Gr}_{k}^{W}(M, F)$ admit the decomposition by strict supports [19, 5.1.3] and their $D_{I}$-components $\left(M_{k, I}, F\right)$ have semisimple monodromies and satisfy:

$$
F_{p} M_{k, I}=\left(V_{0}^{I} V_{<0}^{n \backslash I} M_{k, I}\right) F_{p-q} \mathscr{D}_{X} \text { for } 2 q=|I|-n-k,
$$

where $V_{\alpha}^{J}=n_{i \in J} V_{\alpha}^{(i)}$ and $M_{k, I}=0$ if $|I|-n-k \notin 2 \mathbb{Z}$.

In particular, (3.7.1) implies (3.6.1) for $\mathscr{F}=\mathrm{DR}(M)$, because $\mathrm{Gr}_{k}^{W} E_{I}^{v}=0$ for $|I|$ 
$-n-k \notin 2 \mathbb{Z} . \quad$ Moreover (3.7.1) implies the compatibility of the $n+1$ filtrations $F, V^{(i)}$ on $\mathrm{Gr}_{k}^{W} M$, because the decomposition (3.2.1) for $M(\mu, J)$ gives their splittings compatible to each other. Then, by $[19,1.2 .12],(3.7 .2)$ is equivalent to the surjectivity of

$$
F_{p} V_{v} W_{i} M \longrightarrow F_{p} V_{v} \mathrm{Gr}_{i}^{W} M \text { for any } p, i \in \mathbb{Z}, v \in \mathbb{Q}^{n},
$$

(assuming (3.7.1)), where $V_{v}=\cap V_{v_{J}}^{(j)}$. Moreover, for a decomposition $\operatorname{Gr}_{i}^{W}(M, F)$ $=\oplus\left(M_{i, k}, F\right)$ as in (3.7.1), the condition (3.7.2) is equivalent to:

(3.7.5) for each $i, k$, there exists $u_{i, k} \in F_{p} V_{v} W_{i} M$ such that $\operatorname{Gr}_{i}^{W} u_{i, k}$ belongs to $\left(M_{i, k}\right)^{v} \subset \mathrm{Gr}_{i}^{W} M$ and generates it over $\mathbb{C}$, where $v=\mu+1_{J}, p=q$ if $\left(M_{i, k}\right.$, $F) \cong(M(\mu, J), F[q])$.

In fact, we replace $p$ by $p+|b|$ and apply $x^{a} \partial^{b}$, if we replace $v$ by $v-a+b$ for $a$, $b \in \mathbb{N}^{n}$ such that $a_{i} b_{i}=0$ for any $i$ (because $F_{p}\left(M_{i, k}\right)^{\nu-a+b}=0$ if $p<q+|b|$, where $v, q$ are as in (3.7.5)). Thereore we get for $g=x^{m}$ as in 3.3:

(3.7.6) the vanishing cycle functors along $g$ are well-defined for $(M ; F$, $W) \in \operatorname{MFW}\left(\mathscr{D}_{X}\right)_{g s n c}$ (i.e. $(2.2 .1)$ and (2.3.3) are satisfied), and $\operatorname{MFW}\left(\mathscr{D}_{x}\right)_{g s n c}$ is stable by $\psi_{g}, \phi_{g, 1}$.

In fact, (2.3.3) follows from 3.6, and (2.2.1) is equivalent to the surjectivity of

$$
F_{p} V_{\alpha} W_{i} \tilde{M} \longrightarrow F_{p} V_{\alpha} \mathrm{Gr}_{i}^{W} \tilde{M} \text { for any } i, p \in \mathbb{Z}, \alpha \in \mathbb{Q} \text {. }
$$

which follows from 3.4, (3.5.1-2) and (3.7.5). Then (3.7.1-2) for $\psi_{g} \mathscr{M}, \phi_{g, 1} \mathscr{M}$ follow from an argument similar to the proof of 2.4 , because we have (3.5.3-5) for (3.7.1) and we can verify directly (3.7.2).

Remark. With the notation as above, put $D=\cup_{i \leqq r} D_{i}, g^{\prime}=x^{m^{\prime}}$ for $1 \leqq r \leqq n$, $m^{\prime} \in \mathbb{Z}^{n}$ such that $m_{i}^{\prime}=m_{i}$ for $i>r$ and $m_{i} \neq 0$ for some $i>r$. We define $\left(\mathscr{D}_{X}^{\prime}, F\right)$ $=\left(\mathscr{D}_{X}, F\right)(* D),\left(M^{\prime}, F\right)=(M, F)(* D)$, etc., i.e. $F_{p} M^{\prime}$ is the localization of $F_{p} M$ along $D$. We define $\left(\tilde{M}^{\prime}, F\right)=\left(M^{\prime}\left[\partial_{t}\right], F\right)$ by the same way as above. Then $\tilde{M}^{\prime}$ has the filtration $V^{\prime}$ such that $V_{\alpha}^{\prime} \tilde{M}^{\prime}$ is generated by $V_{m(\alpha-i)} M^{\prime} \otimes \partial_{t}^{i}$ over $\mathscr{D}_{X}^{\prime}[s]$, and we have a canonical isomorphism

$$
\operatorname{Gr}_{\alpha}^{V^{\prime}}\left(\tilde{M}^{\prime}, F\right) \cong\left(\operatorname{Gr}_{\alpha}^{V}(\tilde{M}, F)\right)(* D) \bigotimes_{\mathcal{O}} L\left(\alpha\left(m^{\prime}-m\right)\right)
$$

where $L(v)$ is a left $\mathscr{D}_{X}$-module free of rank one over $\mathcal{O}_{X}(* D)$ with a basis $v$ such that $\left(x_{i} \partial_{i}-v_{i}\right) v=0$. In fact we have the equality in the case $m^{\prime}=m$, then the general case follows from (3.4.3).

As a corollary of (3.7.6) and (3.7.8), we get:

3.8. Theorem. $\mathbb{Q}_{p t}^{H} \in \mathrm{MHM}(p t)^{p}$.

Proof. By definition it is enough to show the following assertion (this argument 
applies to the algebraic case using GAGA):

(3.8.1) Let $X$ be a complex manifold, and $\left\{U_{i}\right\}$ an increasing sequence of finite open sets of $X$ with holomorphic functions $g_{i}$ on $U_{i}$, such that $X \backslash U_{i}$ are locally principal divisors and $g_{i}$ are meromorphic on $X$. Then the vanishing cycle functors along $g_{i}$ are well-defined for $\mathscr{M}_{i}$ and $\left(j_{i}\right)_{i},\left(j_{i}\right)_{*}$ exist for both $\psi_{g_{i}} \mathscr{M}_{i}$, $\phi_{g_{i}, 1} \mathscr{M}_{i}$, where $j_{i}: U_{i} \rightarrow U_{i+1}, \mathscr{M}_{1}=\mathbf{Q}_{U_{0}}^{H}\left[d_{X}\right]$ and $\mathscr{M}_{i+1}$ is obtained by operating $\left(j_{i}\right)_{!}$or $\left(j_{i}\right)_{*}$ after $\psi_{g_{i}}$ or $\phi_{g_{1}, 1}$ to $\mathscr{M}_{i}$.

By Hironaka's desingularization and the stability by projective direct images (cf. 2.14), we may assume $D:=\cup_{i}\left(X \backslash\left(U_{i} \backslash g_{i}^{-1}(0)\right)\right)$ is a normal crossing divisor, because $\mathbf{Q}_{U_{1}}^{H}\left[d_{X}\right]$ is a direct factor of $\mathscr{H}^{0} \pi_{*} \mathbf{Q}_{V_{1}}^{H}\left[d_{X}\right]$ for a resolution $\pi: \tilde{X} \rightarrow X$ with $V_{1}=\pi^{-1}\left(U_{1}\right)$ and $\left(j_{i}\right)_{!},\left(j_{i}\right)_{*}$ are exact (i.e. commute with $\left.{ }^{p} \mathscr{H}^{0}\right)$. By (2.4.3) and 2.11 , the assertion is local on $X$ and it is enough to verify the conditions (2.2.1) and (2.3.3) inductively. Therefore we may forget the $\mathbf{Q}$-structure and assume $X$ is a polydisc $\Delta^{n}$ such that $D \subset \Delta^{n} \backslash\left(\Delta^{*}\right)^{n}$ and $g_{i}=x^{m(i)}$ for $m(i) \in \mathbb{Z}^{n}$, because we can multiply $g_{i}$ by a unit on $X$ (cf. 2.2). If we multiply $g_{i}$ by $x_{k}^{a}$ for $a \in \mathbb{Z}$ such that $x_{k}^{-1}(0) \cap U_{i}=\phi, \psi_{g_{2}}^{\alpha} K_{\mathrm{C}}$ is twisted by a local system as in (3.7.8). If $X=X_{1} \times X_{2}, \quad \mathscr{M}=\mathscr{M}_{1} \otimes \mathscr{M}_{2}$ and $j_{k}$ (resp. $\left.g_{i}\right)$ comes from $X_{1}$ (resp. $X_{2}$ ), we have the commutativity of $\left(j_{k}\right)_{!} j_{k}^{-1},\left(j_{k}\right)_{*} j_{k}^{-1}$ with $\psi_{g_{2}}, \phi_{g_{l}, 1}$ on $\mathscr{M}$ by (3.8.2-5) below. Therefore by changing $m(i)$ and twisting $\mathbf{Q}_{X}^{H}\left[d_{X}\right]$ so that $m(i) \in \mathbf{N}^{n}$ and $\operatorname{dim} g_{i}^{-1}(0) \backslash U_{i+1}<n-1$, we can first take the iteration of $\left(j_{i}\right)_{!} j_{i}^{-1}$ or $\left(j_{i}\right)_{*} j_{i}^{-1}$ and then the iteration of $\psi_{g_{2}}^{\alpha_{2}}$ or $\phi_{g_{i}, 1}$, where we verify only the $\mathscr{D}$ Module part at the stalk of 0 by the above argument and $\psi_{g}^{\alpha}$ means $\operatorname{Gr}_{\alpha}^{V}$ after $\left(i_{g}\right)_{*}$. Then we first get the external product of the $\mathscr{D}$-Module part of $j_{1} \mathbf{Q}_{\Delta^{*}}^{H}$, $j_{*} \mathbb{Q}_{\Delta^{*}}^{H}$ or $M(\alpha, \phi)$ in 3.5 in the one dimensional case, where $j: \Delta^{*} \rightarrow \Delta$. Because these objects belong to $\mathrm{MFW}(\mathscr{D})_{g s n c}$ (cf. 3.7) and their external products to $\operatorname{MFW}\left(\mathscr{D}_{X}\right)_{g s n c}$ (cf. (3.8.2-4) below), the assertion follows from (3.7.6) and (3.7.8).

In the above argument we used the following facts:

(3.8.2) Let $X, \quad Y$ be complex manifolds, and $\mathscr{M} \in \operatorname{MHW}(X)^{(p)}$, $\mathscr{N} \in \operatorname{MHW}(Y)^{(p)}$. Then we have canonical isomorphisms in $\operatorname{MF}_{h}\left(\mathscr{D}_{X \times Y}, \mathbb{Q}\right)$ :

$$
\mathrm{Gr}_{i}^{W}(\mathscr{M} \otimes \mathscr{N})=\oplus \mathrm{Gr}_{j}^{W} \mathscr{M} \otimes \mathrm{Gr}_{i-j}^{W} \mathscr{N} .
$$

Assume $\mathscr{M} \otimes \mathscr{N} \in \mathrm{MHW}(X \times Y)$, and let $g$ be a holomorphic function on $X$. If the vanishing cycle functors along $g$ are well-defined for $\mathscr{M}$, so are they for $\mathscr{M} \otimes \mathscr{N}$ and we have canonical isomorphisms in $\operatorname{MHW}(X \times Y)^{(p)}$ :

$$
\left.\left(\psi_{g} \mathscr{M}\right) \bowtie \mathscr{N} \cong \psi_{g}(\mathscr{M} \bowtie \mathscr{N}) \quad \text { (same for } \phi_{g, 1}\right) .
$$

This follows from the following remarks on the compatible filtrations [19, $\S 1]$ :

(3.8.3) In the category of $\mathcal{O}$-Modules, $\bigotimes$ is exact. Therefore, for compatible $I$ (resp. $J$-) filtrations on $X$ (resp. $Y$ ), we have compatible $(I \cup J)$-filtrations on the 
external product (because the external product of short exact $I$-ple and $J$-ple complexes is a short exact $(I \cup J)$-ple complex, cf. $[19,(1.1 .1)])$.

(3.8.4) For a compatible $I$-filtrations $F_{i}(i \in I)$ and $\{j, k\} \subset I$, such that $F_{j}^{p}=F_{k}^{p}$ $=0$ for $p \gg 0, G_{i}(i \in I)$ are compatible $I$-filtrations, where $G_{i}^{p}=F_{i}^{p}$ for $i \neq j$ and $G_{j}^{p}=\sum\left(F_{j}^{q} \cap F_{k}^{p-q}\right)$. (In fact, $G_{j}^{p}=F_{j}^{p-q}$ on $\operatorname{Gr}_{F_{k}}^{q}$ and the condition [19, (1.2.12.vi)] is satisfied for $G_{i}(i \in I)$, because $\cap F_{i}^{p_{2}} \subset \cap G_{i}^{q_{2}}$ if $p_{i}=q_{i}$ for $i \neq j$ and $p_{j}$ $+q_{k}=q_{j}$.)

As a corollary of (3.8.2) (and 2.11), we get:

(3.8.5) With the notation as in (3.8.2), let $j: U \rightarrow X$ be an open immersion such that $X \backslash U$ is a locally principal divisor. If the vanishing cycle functors along any locally defined $g$ such that $g^{-1}(0)_{\text {red }}=X \backslash U$, and $j_{:} j^{-1}\left(\right.$ or $j_{*} j^{-1}$ ) are welldefined for $\mathscr{M}$, they are well-defined for $\mathscr{M} \otimes \mathscr{N}$ and we have the canonical isomorphism in $\mathrm{MHW}(X \times Y)^{(p)}$ :

$$
\left(j_{!} j^{-1} \mathscr{M}\right) \bowtie \mathscr{N} \cong j_{!} j^{-1}(\mathscr{M} \bowtie \mathscr{N}) \quad\left(\text { same for } j_{*} j^{-1}\right) .
$$

Here $\left(\mathrm{Gr}_{i}^{W} j_{!} j^{-1} \mathscr{M}\right) \otimes\left(\mathrm{Gr}_{k}^{W} \mathcal{N}\right) \in \mathrm{MH}(X \times Y, i+k)$ follows from 2.11 and $\psi_{g, 1} \mathscr{M}$ $凶 \mathscr{N} \in \mathbb{M H W}(X \times Y)$ (locally).

Applying (3.8.2)(3.8.5) to $\mathbb{Q}_{X}^{H}\left[d_{X}\right] \bigotimes \mathscr{M}$ for $\mathscr{M} \in \mathrm{MHM}(p t)^{(p)}$, we have:

3.9. Theorem. MHM $(p t)^{(p)}$ is equivalent to the category of (polarizable) $\mathbb{Q}$ mixed Hodge structures.

Remark. Let $\operatorname{MHM}(X)_{e p}^{(p)}$ be the full subcategory of $\operatorname{MHM}(X)^{(p)}$ whose objects $\mathscr{M}$ satisfies the condition:

$$
\mathscr{M} \otimes \mathscr{N} \in \operatorname{MHM}(X \times Y)^{(p)} \text { for any } Y \text { and } \mathcal{N} \in \operatorname{MHM}(Y)^{(p)} .
$$

Then we have

$$
\operatorname{MHM}(p t)_{e p}^{(p)}=\operatorname{MHM}(p t)^{(p)}
$$

In fact, it is enough to show

$$
\mathscr{M} 区 \mathscr{N} \in \mathrm{MH}(X, i+j)^{(p)} \text { for } \mathscr{M} \in \mathrm{MH}(X, i)^{(p)}, \mathcal{N} \in \mathrm{MH}(p t, j)^{(p)} .
$$

But this follows from the definition of Hodge Modules (because the Hodge structures are stable by $\otimes$ ). We can show in general (cf.3.28 below):

$$
\operatorname{MHM}(X)_{e p}^{p}=\operatorname{MHM}(X)^{p} .
$$

\section{(3.b) Pure Case}

3.10. Let $X$ and $D_{i}$ be as in 3.1. Put $D=\cup_{i \leq r} D_{i}$ for $0 \leq r \leq n=\operatorname{dim} X$, and $U$ 
$=X \backslash D$ with the inclusion $j: U \rightarrow X$. Let $(M, F)$ be a filtered (right) $\mathscr{D}_{U}$-Module such that $F$ is finite and $\operatorname{Gr}_{i}^{F} M$ are locally free coherent $\mathcal{O}_{U}$-Modules (in particular, $M$ is finite free over $\mathcal{O}_{U}$ ). We assume $M$ is quasi-unipotent, i.e. the local monodromies of DR $M$ are quasi-unipotent. We define $j_{*}^{\text {reg }} M\left(\right.$ resp. $\left.j_{!}^{\text {reg }} M\right)$ to be the regular holonomic (right) $\mathscr{D}_{X}$-Modules such that

$$
\mathrm{DR} j_{*}^{\text {reg }} M \cong j_{*} \mathrm{DR} M \quad\left(\text { resp. } \mathrm{DR} j_{!}^{\text {reg }} M \cong j_{!} \mathrm{DR} M\right)
$$

Let $(L, F)$ be the corresponding filtered left $\mathscr{D}_{U}$-Module such that

$$
F_{p} M=\Omega_{U}^{n} \otimes F_{p+n} L
$$

and $\tilde{L}$ Deligne's regular singular meromorphic extension of $L$ with the lattices $\tilde{L}^{\geq a}$ (resp. $\left.\tilde{L}^{>\alpha}\right)$ such that the eigenvalues of res $\nabla$ along $D_{i}(1 \leq i \leq r)$ are contained in $[\alpha, \alpha+1)(\operatorname{resp} .(\alpha, \alpha+1])$. Then we have the natural isomorphisms:

$$
\begin{aligned}
& j_{*}^{\mathrm{reg}} M=\Omega_{U}^{n} \otimes \tilde{L} \\
& \bigcap_{i \leq r} V_{-\alpha-1}^{(i)} j_{*}^{\mathrm{reg}} M=\bigcap_{i \leq r} \Omega_{X}^{n} \otimes V_{(i)}^{\alpha} \tilde{L}=\Omega_{X}^{n} \otimes \tilde{L}^{\geq \alpha}
\end{aligned}
$$

for any $\alpha \in \mathbb{Q}$, where $V^{(i)}$ (resp. $\left.V_{(i)}\right)$ is the filtration $V$ of $j_{*}^{\text {reg }} M$ (resp. $\tilde{L}$ ) along $D_{i}(1 \leq i \leq n)$ such that $x_{i} \partial_{i}-\alpha$ is nilpotent on $\operatorname{Gr}_{\alpha}^{V^{(i)}}\left(\operatorname{resp} . \mathrm{Gr}_{V_{(i)}}^{\alpha}\right)$. Then $V_{(i)}$ on $\widetilde{L}$ and $\widetilde{L}^{\geq 0}$ are the $x_{i}$-adic filtrations for $i>r$ (i.e. $V_{(i)}^{k} \widetilde{L}=x_{i}^{k} \widetilde{L}$, etc.) and

$$
\bigcap_{i \leq r} V_{-\alpha-1}^{(i)} j_{!}^{r e g} M=\Omega_{X}^{n} \otimes \widetilde{L}^{\geq \alpha} \text { for } \alpha>-1 .
$$

We define the filtration $F$ on $\tilde{L}^{\geq \alpha}, \tilde{L}^{>\alpha}$ by:

$$
F_{p} \tilde{L}^{\geq \alpha}=\tilde{L}^{\geq \alpha} \cap j_{*} F_{p} L \quad \text { (same for } \tilde{L}^{>\alpha} \text { ) }
$$

and assume:

(3.10.4) $F_{P} \tilde{L}^{\geq \alpha}$ are coherent over $\mathcal{O}_{X}$,

(3.10.5) $n+1$ filtrations $F, V_{(i)}(1 \leq i \leq n)$ on $\tilde{L}^{\geq \alpha}$ are compatible.

Then these two conditions are independent of $\alpha$ (because $\left(F_{p} \widetilde{L}^{\geq \alpha}\right) x_{1} \cdots x_{r}$ $=F_{p} \tilde{L}^{\geq \alpha+1}$ ), and for $\alpha=0$ they imply:

(3.10.6) We have a free $\mathcal{O}_{X}$-basis $\left\{u_{p, \alpha, k}\right\}$ of $\tilde{L}^{\geq 0}$ on a neighborhood of 0 indexed by $p \in \mathbb{Z}, \alpha \in(\mathbb{Q} \cap[0,1))^{r}$ and $1 \leq k \leq m(p, \alpha)$ such that

$$
\begin{aligned}
& F_{q} \tilde{L}^{\geq 0}=\oplus_{p \leq q} \mathcal{O}_{X} u_{p, \alpha, k} \\
& V_{(i)}^{\beta} \tilde{L}^{\geq 0}=\oplus \mathcal{O}_{X} x_{i}^{-\min \left(\left[\alpha_{\imath}-\beta\right], 0\right)} u_{p, \alpha, k}
\end{aligned}
$$

where we put $\alpha_{i}=0$ for $i>r$ in the second formula.

In fact, (3.10.4-5) for $\alpha=0$ imply the existence of a free basis $\left\{u_{p, \alpha, k}\right\}$ indexed as 
above, such that $u_{p, \alpha, k} \in F_{p} V^{\prime \alpha} L^{\geq 0}$ and $\left\{u_{p, \alpha, k}\right\}_{1 \leq k \leq m(p, \alpha)}$ is a C-basis of $\mathrm{Gr}_{F}^{p} \mathrm{Gr}_{V^{\prime}}^{\alpha} \mathrm{Gr}_{V \prime \prime}^{0} \tilde{L}^{\geq 0}$ (where $V^{\prime \alpha}=\bigcap_{i \leq r} V_{(i)}^{\alpha_{2}}, \mathrm{Gr}_{V^{\prime}}^{\alpha}=\prod_{i \leq r} \mathrm{Gr}_{V_{(l)}}^{\alpha_{2}}, \mathrm{Gr}_{V^{\prime \prime}}^{0}=\prod_{i>r} \mathrm{Gr}_{V_{(l)}}^{0}$ ), because $\left(F_{p} V^{\alpha} \tilde{L}^{\geq 0}\right) x_{i}=F_{p} V^{\alpha+1_{i}} \tilde{L}^{\geq 0}$ if $\alpha_{i} \geq 0$, and a finite $\mathcal{O}_{X, 0}$-module $M$ is free iff the $x_{j}$-adic filtrations $V_{(j)}$ are compatible filtrations and $x_{j}:\left(M, V_{(i)}(i \neq j)\right)$ $\stackrel{\sim}{\rightarrow}\left(V_{(j)}^{1} M, V_{(i)}(i \neq j)\right)$. Conversely (3.10.6) implies clearly (3.10.4-5) on neighborhood of 0 . If the monodromies are unipotent, (3.10.6) is equivalent to:

$$
\mathrm{Gr}_{p}^{F} L^{\geq 0} \text { are finite free over } \mathcal{O}_{X} \text { on a neighborhood of } 0 .
$$

Note that the condition (3.10.6) is stable by the direct image for a finite morphism ramified along $D$ (because we have a basis $\left\{x^{v} u_{p, \alpha, k}\right\}$ on the image). Therefore (3.10.4-6) are satisfied, if $(L, F)$ underlies a polarizable variation of Hodge structure, by the well-known result of Schmid.

We now define the filtrations $F, G$ on $j_{*}^{\text {reg }} M$ by

$$
\begin{aligned}
& F_{p} j_{*}^{\mathrm{reg}} M=\sum\left(\Omega_{X}^{n} \otimes F_{i} \tilde{L}^{\geq-1}\right) F_{p-i} \mathscr{D}_{X} \\
& G_{p} j_{*}^{\mathrm{reg}} M=\left(\Omega_{X}^{n} \otimes \tilde{L}^{\geq-1}\right) F_{p} \mathscr{D}_{X}
\end{aligned}
$$

and on $j_{!}^{\text {reg }} M$ by replacing $\tilde{L}^{\geq-1}$ with $\tilde{L}^{>-1}$. We define the filtered differential complexes $[19, \S 2]$ by:

$$
\begin{aligned}
& K_{*}(L, F)=\left(\Omega_{X}^{\circ}(\log D), F\right) \otimes\left(\tilde{L}^{\geq 0}, F\right)[n] \\
& K_{!}(L, F)=\left(\Omega_{X}^{*}(\log D), F\right) \otimes\left(\tilde{L}^{>0}, F\right)[n],
\end{aligned}
$$

where the filtration $F$ on $\Omega_{X}^{*}(\log D)$ is defined by $F_{p}=\sigma_{\geq-p}$ (cf. [9]) and their underlying complexes are the Koszul complexes for the action of $x_{i} \partial_{i}(i \leq r), \partial_{i}(i$ $>r$ ) on $\tilde{L}^{\geq 0}, \tilde{L}^{>0}$ using the coordinates $\left(x_{1}, \ldots, x_{n}\right)$. We define filtration $G$ on $\mathrm{DR}^{-1} K_{*(!)}(L)$ the underlying complex of $\mathrm{DR}^{-1} K_{*(!)}(L, F)(\mathrm{cf} .[19, \S 2])$ by:

$$
G_{p}\left(\mathrm{DR}^{-1} K_{*(!)}(L)\right)^{-j}=\Omega_{X}^{n-j}(\log D) \otimes \tilde{L}^{\geq 0(>0)} \otimes F_{p-j} \mathscr{D}_{X}
$$

and the filtration $V^{(i)}$ as follows: for $D^{\prime}=U_{1 \leq i \leq n} D_{i}$, put

$$
\begin{gathered}
K_{*}^{\prime}(L)=\Omega_{X}\left(\log D^{\prime}\right) \otimes \tilde{L}^{\geq 0}[n] \\
V_{\alpha}^{(i)}\left(\mathrm{DR}^{-1} K_{*}^{\prime}(L)\right)^{-j}=\sum \Omega_{X}^{n-j}\left(\log D^{\prime}\right) \otimes V_{(i)}^{k-\alpha} \tilde{L}^{\geq 0} \otimes V_{k}^{(i)} \mathscr{D}_{X},
\end{gathered}
$$

then the filtration $V^{(i)}$ on $\mathrm{DR}^{-1} K_{*(!)}(L)$ is induced by the natural inclusion into $\mathrm{DR}^{-1} K_{*}^{\prime}(L)$. We define

$$
\left(j_{! *}^{\mathrm{reg}} M, F\right)=\operatorname{Coim}\left(\left(j_{!}^{\mathrm{reg}} M, F\right) \longrightarrow\left(\left(j_{*}^{\mathrm{reg}} M, F\right)\right)\right.
$$

so that

$$
F_{p} j_{! *}^{\mathrm{reg}} M=\sum\left(\Omega_{X}^{n} \otimes F_{i} \tilde{L}^{>-1}\right) F_{p-i} \mathscr{D}_{X} \text { in } j_{!_{*}}^{\mathrm{reg}} M .
$$

3.11. Proposition. With the notations and the assumptions as above, on a neighborhood of 0 , we have the followings: 
(i) the $n+2$ filtrations on each component of $\left(\mathrm{DR}^{-1} K_{*(!)}(L, F) ; G, V^{(i)}\right)$ are compatible and this $n+2$ filtered complex is strict $[19,1.2 .2]$,

(ii) the above complex gives a resolution of $\left(j_{*(!)} M ; F, G, V^{(i)}(1 \leq i \leq n)\right)$ so that the $n+2$ filtrations on $j_{*(!)} M$ are compatible,

(iii) we have the filtered isomorphisms:

$$
\begin{aligned}
& x_{1}:\left(V_{\alpha}^{(i)} j_{*(!)}^{\mathrm{reg}} M ; F, V^{(1)}, \ldots, V^{(i)}, \ldots, V^{(n)}\right) \\
& \stackrel{\sim}{\longrightarrow}\left(V_{\alpha-1}^{(i)} j_{*(!)}^{\mathrm{reg}} M ; F, V^{(1)}, \ldots, V^{(i)}[1], \ldots, V^{(n)}\right)(\alpha<0) \\
& \partial_{i}:\left(\mathrm{Gr}_{\alpha}^{V^{(2)}} j_{*(!)}^{\mathrm{reg}} M ; F, V^{(j)}(j \neq i)\right) \\
& \stackrel{\sim}{\longrightarrow}\left(\mathrm{Gr}_{\alpha+1}^{V^{(2)}} j_{*(!)}^{\mathrm{reg}} M ; F[-1], V^{(j)}(j \neq i)\right)(\alpha>-1),
\end{aligned}
$$

and (3.11.1)(resp. (3.11.2)) holds also for $\alpha=0$ (resp. - 1) and $i \leq r$ (where $j_{*(!)}^{\mathrm{reg}} M$ means $j_{*}^{\text {reg }} M$ (resp. $j_{!}^{\text {reg }} M$ ) and same for $K_{*(!)}$.

Proof. By definition, (DR $\left.{ }^{-1} K_{*(!)}(L, F) ; G, V^{(i)}\right)$ is the Koszul complex for the (left) actions of $x_{j} \partial_{j}(j \leq r), \partial_{j}(j>r)$ on $\tilde{L}^{\geq 0} \otimes \mathscr{D}_{X}$ (resp. $\tilde{L}^{>0} \otimes \mathscr{D}_{X}$ ) where the filtrations are shifted appropriately. Therefore, for the first assertion of (i), it is enough to show the compatibility on

$$
\tilde{L}^{\geq 0(>0)} \otimes \mathscr{D}_{X}=\oplus_{\nu} \tilde{L}^{\geq 0(>0)} \otimes \partial^{v} .
$$

But the filtrations are compatible with this decomposition, and the assertion follows from (3.10.5). For the second assertion of (i) it is enough to show the strictness of

$$
\left(\mathrm{Gr}^{F} \mathrm{Gr}^{G} \mathrm{DR}^{-1} K_{*(!)}(L) ; V^{(i)}\right)
$$

and $H^{j}$ of (3.11.3) is zero for $j \neq 0$, by $[19,1.3 .6]$. But, by the basis $\left\{u_{p, \alpha, k}\right\}$ in (3.10.6), (3.11.3) is a direct sum of Koszul complex for the action of $x_{i} \xi_{i}(i \leq r)$, $\xi_{i}(i>r)$ on $\mathcal{O}_{X}[\xi]$, where $\xi_{i}=\operatorname{gr} \partial_{i}$ and $V^{(i)}$ are shifted according to $\alpha_{i}$ of $\alpha$ (if this factor corresponds to $u_{p, \alpha, k}$ ) and depending on the image or the source of $\partial_{i}$ if $i>r$, so that $x_{i} \partial_{i}, \partial_{i}$ are strict. We can verify that (3.11.3) is strict and $H^{j}=0$ for $j \neq 0$ by taking inductively the cohomology for the action of $x_{i} \partial_{i}, \partial_{i}$, cf. [19, $\S 1.3]$. For (ii), we verify that the isomorphism:

$$
\Omega_{X}^{n}(\log D) \otimes \tilde{L}^{\geq 0} \cong \bigcap_{i \leq r} V_{0}^{(i)} j_{*}^{\mathrm{reg}} M(\mathrm{cf} .(3.10 .1))
$$

and (3.10.2) induce the natural morphisms:

$$
\left(\mathrm{DR}^{-1} K_{*(!)}(L, F) ; G, V^{(i)}\right) \longrightarrow\left(j_{*(!)}^{\mathrm{reg}} M ; F, G, V^{(i)}\right)
$$

using the expression as Koszul complex as above (because $(d x / x) x_{i} \partial_{i}=0$ ). Then it is enough to show that (3.11.4) is a quasi-isomorphism forgetting the filtrations by (i) (and by definition of the filtrations). Using a filtration of $L$, we can reduce 
to the case $\operatorname{rank} L=1$. Then the assertion is clear. Now (iii) follows from (i) (ii), because (3.11.1-2), etc. are verified for each component of $\mathrm{DR}^{-1} K_{*(!)}(L)$.

3.12. Proposition. With the notations and the assumptions as in 3.10, assume the morphisms

$$
N_{J}: \operatorname{Gr}_{V^{\prime}}^{\alpha} \operatorname{Gr}_{V \prime \prime}^{0}\left(\tilde{L}^{\geq 0}, F\right) \longrightarrow \operatorname{Gr}_{V^{\prime}}^{\alpha} \operatorname{Gr}_{V \prime \prime}^{0}\left(\tilde{L}^{\geq 0}, F\right)
$$

are strict for any $\alpha \in([0,1) \cap \mathbb{Q})^{r}$ and $J \subset\{1, \ldots, r\}$, where $N_{J}=\prod_{i \in J} N_{i}$ and $N_{i}$ $=x_{i} \partial_{i}-\alpha_{i}(1 \leq i \leq r)$. Then, on the stalk at 0 , we have the followings:

(i) the natural morphism:

$$
\left(j_{!}^{\mathrm{reg}} M ; F, V^{(i)}(1 \leq i \leq n)\right) \rightarrow\left(j_{*}^{\mathrm{reg}} M ; F, V^{(i)}(1 \leq i \leq n)\right)
$$

is strict so that the $n+1$ filtrations are compatible on $j_{! *}^{\mathrm{reg}} M$ the image of (3.12.2),

(ii) $\left(j_{*}^{\mathrm{reg}} M, F\right)$ is Cohen-Macaulay, and (3.11.1-2) hold for $j_{: *}^{\mathrm{reg}} M$,

(iii) for $v \in([-1,0) \cap \mathbb{Q})^{n}$ and $J \subset \bar{n} \backslash \bar{v}$ (cf. 3.1), the morphism:

$$
\partial_{J}: \mathrm{Gr}_{v}^{V}\left(j_{! *} M, F\right) \rightarrow \mathrm{Gr}_{v+1_{J}}^{V}\left(j_{! *} M, F[-|J|]\right)
$$

is a strict epimorphism whose kernel coincides with $\operatorname{Ker} N_{J}$ (i.e. the target of (3.12.3) is identified with $\operatorname{Im} N_{J}$ ).

Proof. This follows from the next two propositions. In fact the assumption of 3.13 is satisfied by the strictness of (3.12.1) (combined with $\mathrm{Gr}^{V}$ of (3.11.1-2)), because we have a diagram:

$$
\begin{aligned}
& \mathrm{Gr}_{v}^{V} j_{!} M \stackrel{\sim}{\longrightarrow} \mathrm{Gr}_{v}^{V} j_{*} M
\end{aligned}
$$

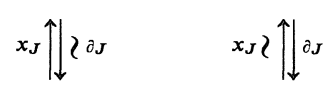

$$
\begin{aligned}
& \mathrm{Gr}_{v+1_{J}}^{V} j_{!} M \longrightarrow \mathrm{Gr}_{v+1_{J}}^{V} j_{*} M,
\end{aligned}
$$

and the first assertion of (ii) follows from 3.14 using the same argument as in the proof of $[19,5.1 .13]$ inductively. The other assertions are trivial.

3.13. Proposition. Let $\left(M^{\circ}, F\right)$ be a bounded complex of filtered $\mathscr{D}_{X}$-modules such that $M^{j} \in M\left(\mathscr{D}_{X}\right)_{\text {rqunc }}$ (cf. 3.2). Then the $n+1$ filtered complex $\left(M^{\circ} ; F, V^{(i)}\right)$ is strict, if the following conditions are satisfied:

(3.13.1) the $n+1$ filtrations $F, V^{(i)}$ are compatible on each $M^{j}$,

(3.13.2) the conditions (3.11.1-2) hold for each $M^{j}$,

(3.13.3) $\operatorname{Gr}_{v}^{V}\left(M^{\bullet}, F\right)$ is strict for any $v \in([-1,0] \cap \mathbf{Q})^{n}$.

Proof. By induction on $n$. The case $n=1$ follows from [19, 3.3.3-5]. In general, the assumptions are satisfied on each $\mathrm{Gr}_{\alpha}^{V^{(n)}} M^{*}$ at the origin, and in the proof of [loc. cit], we replace $M$ by $(n-1)$-ple diagram of short exact sequences 
associated to any $n-1$ subobjects $V_{\alpha_{1}}^{(i)} M(i \neq n)(\mathrm{cf} .[19,1.1 .13])$, and complete the diagram by adding the term for $F_{p} / F_{q}$ whenever the inclusion $F_{p} \rightarrow F_{q}$ appears. Then we get the assertion, where we use the fact that the $x_{n}$-adic completion (on finite modules) is exact and fully faithful.

3.14. Proposition. Put $(X, 0)=\left(\mathbb{C}^{n}, 0\right),(Y, 0)=(X, 0) \times(\mathbb{C}, 0), \mathscr{D}^{\prime}=\mathscr{D}_{X, 0}$ and $\mathscr{D}=\mathscr{D}_{Y, 0}$. Let $(M, F)$ be a filtered $(\mathscr{D}, F)$-module of finite type (i.e. $\mathrm{Gr}^{F} M$ is a finite graded $\mathrm{Gr}_{\mathscr{D}}^{F}$-module). Assume $(M, F)$ is quasi-unipotent and regular along $X$, i.e. $M$ has the filtration $V$ along $X$ such that $\bar{M}_{\alpha}=0$ for $m \alpha \notin \mathbf{Z}, \operatorname{Gr}^{F} \bar{M}_{\alpha}$ are finite over $\mathrm{Gr}^{F} \mathscr{D}^{\prime}$, and

$$
\begin{gathered}
t:\left(\bar{M}_{\alpha}, F\right) \stackrel{\sim}{\rightarrow}\left(\bar{M}_{\alpha-1}, F\right) \quad \text { for } \alpha<0 \\
\partial_{t}:\left(\bar{M}_{\alpha}, F\right) \stackrel{\sim}{\longrightarrow}\left(\bar{M}_{\alpha+1}, F[-1]\right) \text { for } \alpha>-1
\end{gathered}
$$

where $\bar{M}_{\alpha}=\mathrm{Gr}_{\alpha}^{V} M$. Then $\operatorname{Gr}^{F} \mathrm{Gr}^{V} M$ is Cohen-Macaulay (i.e. $\operatorname{Ext}_{\mathrm{Gr}^{F} \mathrm{Gr}^{V} \mathscr{D}}^{j}$ $\left(\mathrm{Gr}^{F} \mathrm{Gr}^{V} M, \mathrm{Gr}^{F} \mathrm{Gr}^{V} \mathscr{D}\right)=0$ except for one $\left.j\right)$, if $\mathrm{Gr}^{F} \bar{M}_{\alpha}$ are Cohen-Macaulay over $\mathrm{Gr}^{F} \mathscr{D}^{\prime}$, and $\operatorname{dim}_{\mathrm{Gr}^{F} \mathscr{D}^{\prime}} \mathrm{Gr}^{F} \bar{M}_{\alpha}$ is independent of $\alpha$.

Proof. Put $(\bar{M}, F)^{\lambda}=\bigoplus_{\alpha \in \lambda}\left(\bar{M}_{\alpha}, F\right)$ for $\lambda \in \mathbf{Q} / \mathbb{Z}$ so that $\operatorname{Gr}^{V}(M, F)=\oplus(\bar{M}$, $F)^{\lambda}$. Then we have a filtered quasi-isomorphism: $C(\bar{M}, F)^{\lambda} \rightarrow(\bar{M}, F)^{\lambda}$, where $C(\bar{M}, F)^{\lambda}$ is defined by the mapping cone:

$$
\begin{aligned}
& C\left(\left(\bar{M}_{0}, F \tilde{)} \oplus\left(\overline { M } _ { - 1 } F [ 1 ] \tilde { ) } \longrightarrow \left(\bar{M}_{-1}, F \tilde{)} \oplus\left(\bar{M}_{0}, F \tilde{)}\right) \text { for } \lambda=\mathbb{Z}\right.\right.\right.\right. \\
& C\left(\left(\bar{M}_{\alpha}, F\right) \longrightarrow\left(\bar{M}_{\alpha}, F\right) \tilde{)}\right) \text { otherwise where } \lambda=\alpha+\mathbb{Z},-1<\alpha<0 .
\end{aligned}
$$

Here $\left(\bar{M}_{\alpha}, F\right):=\left(\bar{M}_{\alpha}, F\right) \otimes_{\mathrm{C}}(A, F),(A, F):=\mathrm{Gr}^{V}\left(\mathscr{D}_{\mathbf{C}, 0}, F\right)=\left(\mathbf{C}\left[t, \partial_{t}\right], F\right)$ and the morphisms in the mapping cones are defined by:

$$
\begin{aligned}
& (u \otimes P, v \otimes Q) \rightarrow\left(-u t \otimes P+v \otimes \partial_{t} Q, u \otimes t P-v \partial_{t} \otimes Q\right) \quad(\alpha \in \mathbb{Z}) \\
& (u \otimes P) \longmapsto\left(-u\left(t \partial_{t}-\alpha\right) \otimes P+u \otimes\left(t \partial_{t}-\alpha\right) P\right) \quad \text { (otherwise) }
\end{aligned}
$$

(In fact, for the proof of filtered quasi-isomorphism, we can reduce to the case where the action of $t \partial_{t}-\alpha$ is zero $(-1 \leq \alpha \leq 0)$ and $\bar{M}_{-1}$ or $\bar{M}_{0}$ is zero, using a filtration on $\bar{M}_{\alpha}$, because $\otimes(A, F)$ is exact.) Now let $\left(L_{\alpha}, F\right) \rightarrow\left(\bar{M}_{\alpha}, F\right)$ be a resolution by a bounded complex of finite free filtered $\mathscr{D}^{\prime}$-modules $(-1 \leq \alpha \leq 0)$. We may assume the morphisms $t, \partial_{t}$ and $t \partial_{t}-\alpha$ are extended to $\left(L_{\alpha}, F\right)$. Then we get a free resolution of $(\bar{M}, F)^{\lambda}$, by replacing $\left(\bar{M}_{\alpha}, F\right)$ with $\left(L_{\alpha}, F\right)$ in the above construction, because $\otimes(A, F)$ is exact. We verify the above construction is compatible with dual, i.e. the dual of the above free resolutions are the mapping cones associated to $\mathbf{D}\left(L_{\alpha}, F\right)$. Then we get the assertion using the same argument as above, because $\mathbf{D}\left(L_{\alpha}, F\right)$ is strict and $\mathscr{H}^{j} \mathbf{D}\left(L_{\alpha}, F\right)=0$ except for one $j$ (independent of $\alpha$ ) by assumption. 
3.15. Remark. 1) In the case of polarizable variation of Hodge structures, the assumtion (3.12.1) is satisfied by [6]. More generally, let $X$ be a complex manifold, $D$ a normal crossing divisor on $X$ and put $j: U=X \backslash D \rightarrow X$. Assume $(L, F)$ underlies a polarizable variation of Hodge structure $\mathbb{H}$ of weight $w$ on $U$. Then we can define $\left(j_{*}^{\text {reg }} M, F\right)$, etc. (globally) on $X$ so that $\left(j_{* *}^{\text {reg }} M, F\right)$ is Cohen-Macaulay and the polarization induces isomorphisms:

$$
\begin{aligned}
& \mathbb{D}\left(j_{*}^{\mathrm{reg}} M, F\right) \cong\left(j_{!}^{\mathrm{reg}} M, F[w+\operatorname{dim} X]\right) \\
& \mathbb{D}\left(j_{*}^{\mathrm{reg}} M, F\right) \cong\left(j_{*}^{\mathrm{reg}} M, F[w+\operatorname{dim} X]\right)
\end{aligned}
$$

by $3.11-12$, because $(3.15 .1)$ holds by replacing $\left(j_{*(!)}^{\text {reg }} M, F\right)$ with $K_{*(!)}(L, F)$ by definition and we can apply 3.12 at every point of $X$.

2) Assume furthermore $X$ is compact $\mathrm{Kähler,} \mathrm{and} \mathrm{let}\left(\mathscr{L}_{(2)}(\mathrm{H}), F\right)$ be the filtered $L^{2}$-complex on $X$ associated to $H$ (cf. [8][13][15]). Then Kashiwara and Kawai constructed a morphism in $D^{b} F^{f}\left(\mathcal{O}_{X}\right.$, Diff):

$$
\mathrm{DR}\left(j_{! *}^{\mathrm{reg}} M, F\right) \rightarrow\left(\mathscr{L}_{(2)}(\mathrm{H}), F\right)[\operatorname{dim} X],
$$

inducing an isomorphism on $U$ (forgetting $F$ ), cf. [15]. To show that (3.15.2) is an isomorphism after taking $\mathbb{R} \Gamma(X, *)$ (cf. [loc. cit]), it is enough to verify the well-definedess of the morphisms (induced by the polarization):

$$
\begin{aligned}
\left(\mathscr{L}_{(2)}(\mathrm{H}), F\right) \otimes\left(\mathscr{L}_{(2)}(\mathbf{H}), F\right) & \longrightarrow\left(\mathscr{L}_{(1)}(\mathbf{C}), F[-w]\right) \\
& \longrightarrow(\mathscr{D} b, F[-w])
\end{aligned}
$$

where $(\mathscr{D} b, F)=\left(\tilde{I}_{X}, F[-\operatorname{dim} X]\right)[-2 \operatorname{dim} X]$ in $[19,2.5 .1]$ and $\mathscr{L}_{(1)}$ is with respect to the Poincare metric. In fact, combined with the morphism $\left(\widetilde{I}_{X}, F\right)$ $\rightarrow\left(\tilde{K}_{X}, F\right)$ [loc. cit], (3.15.3) induces a morphism in $D^{b} F^{f}\left(\mathcal{O}_{X}\right.$, Diff):

$$
\left(\mathscr{L}_{(2)}(\mathbf{H}), F\right)[\operatorname{dim} X] \rightarrow \mathbb{D}\left(\left(\mathscr{L}_{(2)}(\mathbf{H}), F\right)[\operatorname{dim} X]\right)(-w-\operatorname{dim} X),
$$

cf. $[19,(2.4 .11 .2)]$, and this gives a left inverse of (3.15.3) using (3.15.2) (and the dual functor $\mathbb{D})$, i.e. $\left(\mathrm{DR}\left(j_{! *}^{\mathrm{reg}} M, F\right)\right.$ is a direct factor of $\left(\mathscr{L}_{(2)}(\mathbb{H}), F\right)[\operatorname{dim} X]$, by the octahedral axiom. Then the strictness of $\mathbb{R} \Gamma\left(X,\left(\mathscr{L}_{(2)}(\mathbb{H}), F\right)\right)$ implies that of $\mathbb{R} \Gamma\left(X, \operatorname{DR}\left(j_{!_{*}}^{\mathrm{reg}} M, F\right)\right)$ and we get the desired isomorphism. (The advantage of this argument is that we don't have to show the self-duality of $\left(\mathscr{L}_{(2)}(\mathbb{H})\right.$, $F)[\operatorname{dim} X]$ in $D^{b} F^{f}\left(\mathcal{O}_{X}\right.$, Diff).) Note that, if 1 does not belong to the eigenvalues of the local monodromies, $(3.15 .3)$ is trivial, because $\left(j !{ }^{\text {reg }} M, F\right)$ $=\left(j_{! *}^{\mathrm{reg}} M, F\right)=\left(j_{*}^{\mathrm{reg}} M, F\right)$ in this case.

3.16. With the notation of 3.2 (e.g. $\mathscr{D}_{X}=\mathscr{D}_{X, 0}$ ), let $(M, F)$ be a filtered $\mathscr{D}_{X^{-}}$ module. We say $(M, F)$ is quasi-unipotent and regular of normal crossing type, if :

$$
M \in M\left(\mathscr{D}_{X}\right)_{r n c q u},
$$


the $n+1$ filtrations $F, V^{(i)}$ are compatible,

Here $F$ is assumed to satisfy $\cup F_{p} M=M$ and $F_{p} M=0$ for $p \ll 0$ as always (cf. [19,2.1.1]). In particular we have

$$
F \text { is finite on } V_{0} M\left(=\cap V_{0}^{(i)} M\right) .
$$

We shall denote by $M F\left(\mathscr{D}_{X}\right)_{r n c q u}$ the category of the above filtered $\mathscr{D}_{X^{-}}$ modules. Then it is an exact category with respect to the $n+1$ filtrations. Note that (3.16.3-4) are equivalent to (3.11.1-2) under the assumption (3.16.1). By the same argument as the proof of 3.12 , ii), we have

$$
(M, F) \in \operatorname{MF}\left(\mathscr{D}_{X}\right)_{r n c q u} \text { is Cohen-Macaulay, }
$$

because $(M, F)$ is finitely generated by the argument below:

Let $(M, F) \in \operatorname{MF}\left(\mathscr{D}_{X}\right)_{r n c q u}$ and $u_{p, v, k} \in F_{p} V_{v} M$ such that $\left\{u_{p, v, k}\right\}_{1 \leq k \leq m(p, v)}$ is a basis of $\operatorname{Gr}_{p}^{F} \operatorname{Gr}_{v}^{V} M$ for any $p \in \mathbb{Z}, v \in(\mathbf{Q} \cap[-1,0])^{n}$. Then $\left\{u_{p, v, k}\right\}$ generate $F$; more precisely

$$
F_{p} V_{\mu} M=\sum u_{q, v, k} x^{a} \partial^{b} \mathcal{O}_{X} \text { for } p \in \mathbb{Z}, \mu \in(\mathbf{Q} \cup\{+\infty\})^{n},
$$

where the summation is taken over $q \in \mathbb{Z}, v \in(\mathbf{Q} \cap[-1,0])^{n}, 1 \leq k \leq m(q, v), a$, $b \in \mathbb{N}^{n}$ satisfying: $q+|b| \leq p ; v_{i}-a_{i}+b_{i} \leq \mu_{i}$ for any $i ; a_{i}=0$ if $v_{i}=0$ or $\mu_{i} \geq v_{i} ; b_{i}=0$ if $v_{i}=-1$ or $\mu_{i} \leq v_{i} ; \mu_{i}-1<v_{i}-a_{i}$ if $\mu_{i} \leq v_{i}$. In fact, the assertion is clear by the same argument as in (3.10.6), if $\mu_{i}<0$ for any $i$. Then we can proceed by induction on $\mu$ and $\operatorname{dim} X$, because $\operatorname{MF}\left(\mathscr{D}_{X}\right)_{\text {rncqu }}$ is stable by $\mathrm{Gr}_{\alpha}^{V^{(2)}}$ for any $i, \alpha$. (Note that we can replace $\mathcal{O}_{X}$ by $\mathbb{C}\left\{x_{I(v, b)}\right\}$ in (3.16.7), where $\left.I(v, b)=\left\{i ; v_{i} \neq 0, b_{i}=0\right\}.\right)$ In particular we get:

$$
F_{p} V_{\mu} M=\sum\left(F_{p-|b|} V_{v} M\right) \partial^{b} \text { for any } p, \mu,
$$

where the summation is taken over $v \in \mathbb{Q}^{n}, b \in \mathbf{N}^{n}$ satisfying: $v_{i}=0$ if $b_{i} \leq \mu_{i}$; $v_{i}>-1$ and $v_{i}+b_{i}=\mu_{i}$ if $b_{i}>\mu_{i}>0 ; b_{i}=0$ and $v_{i}=\mu_{i}$ if $\mu_{i} \leq 0$.

Conversely, let $F$ be a finite filtration by $\mathcal{O}_{X}$-submodule of $M_{0}:=V_{0} M$ for $M \in M\left(\mathscr{D}_{X}\right)_{r n c q u}$, where $V_{0} M=\cap V_{0}^{(i)} M$. Assume:

$$
\text { the } n+1 \text { filtrations } F, V^{(i)} \text { are compatible on } M_{0} \text {, }
$$

and define $F_{p} M=\sum\left(F_{p-j} M_{0}\right) F_{j} \mathscr{D}_{X}$. Then:

$$
\begin{aligned}
& \left(F_{p} V_{\alpha}^{(i)} M_{0}\right) x_{i}=F_{p} V_{\alpha-1}^{(i)} M_{0} \text { for } \alpha<0 \\
& \left(F_{p} V_{-1}^{(i)} M_{0}\right) \partial_{i} \subset F_{p+1} V_{0}^{(i)} M_{0}
\end{aligned}
$$

$$
(M, F) \in \operatorname{MF}\left(\mathscr{D}_{X}\right)_{r n c q u} \text { and } F_{p} M \cap M_{0}=F_{p} M_{0} .
$$


In fact, we define $F$ on $M_{\mu}:=V_{\mu} M$ for $\mu \in(\mathbb{Q} \cup\{+\infty\})^{n}$ by

$$
F_{p} M_{\mu}=\sum\left(F_{p-|b|} V_{v} M_{0}\right) \partial^{b}
$$

where the summation is taken over $v, b$ as in (3.16.8). Then (3.16.12) follows from (3.16.13) and the next two assertions:

$$
\text { the } n+1 \text { filtrations } F, V^{(i)} \text { are compatible on } M_{\mu} \text {, }
$$$$
\left(M_{v} ; F, V^{(i)}\right) \rightarrow\left(M_{\mu} ; F, V^{(i)}\right) \text { are strict monomorphisms as } n+1
$$

filtered morphisms, if $v_{i} \leq \mu_{i}$ for $1 \leq i \leq n$.

Here note that (3.16.14-15) are clear by definition if $\mu_{i} \leq 0$ for any $i$ (because $F_{p} M_{\mu}=F_{p} V_{\mu} M_{0}$ in this case), and under the assumption (3.16.14), (3.16.15) is equivalent to $F_{p} M_{v}=M_{v} \cap F_{p} M_{\mu}$ (by $[19,1.2 .12]$, if $v_{i}=\mu_{i}(i \neq j)$ ). We shall prove (3.16.14-15) by induction on $\sum \max \left(\mu_{i}, 0\right)$ and $n=\operatorname{dim} X$. We take $1 \leq j \leq n, b \in \mathbf{N}$ and $\mu^{\prime} \in \mathbb{Q}^{n}$ such that $\mu_{j}>0,-1<\mu_{j}-b \leq 0, \mu_{i}=\mu_{i}^{\prime}(i \neq \cdot j)$, $\mu_{j}>\mu_{j}^{\prime}$ and $\operatorname{Gr}_{\mu_{j}}^{V^{(j)}} M=V_{\mu_{j}}^{(j)} M / V_{\mu_{j}^{\prime}}^{(j)} M$. Then, for (3.16.14), it is enough to show the exact sequences for $v=\left\{v_{i}(i \neq j)\right\} \in \mathbb{Q}^{n-1}$ :

$$
0 \longrightarrow F_{p} V_{v} M_{\mu^{\prime}} \longrightarrow F_{p} V_{v} M_{\mu} \longrightarrow F_{p} V_{v} \mathrm{Gr}_{\mu_{J}}^{V^{(J)}} M_{\mu} \longrightarrow 0
$$

by [loc. cit], because $(3.16 .14)$ is verified for

$$
\left(\mathrm{Gr}_{\mu_{J}-b}^{V} M_{\mu-b \cdot 1,}, F\right) \stackrel{\partial_{\mu}^{k}}{\longrightarrow}\left(\mathrm{Gr}_{\mu_{J}}^{V} M_{\mu}, F[-k]\right)
$$

by inductive hypothesis on $\operatorname{dim} X$. But the surjectivity of the second morphism in (3.16.16) is clear by (3.16.13) for $\mathrm{Gr}_{\mu_{j}-b}^{V} M_{\mu-b \cdot 1,}$. Therefore it remains to show $F_{p} M_{\mu^{\prime}}=M_{\mu^{\prime}} \cap F_{p} M_{\mu}$, but this is reduced to

$$
F_{p-b} M_{\mu^{\prime}-b \cdot 1,}=M_{\mu^{\prime}-b \cdot 1,} \cap F_{p-b} M_{\mu-b \cdot 1,}
$$

by definition of $F$, because $\partial_{j}: \mathrm{Gr}_{\alpha}^{V^{(j)}} \rightarrow \mathrm{Gr}_{\alpha+1}^{V^{(J)}}$ is injective for $\alpha>-1$. Then the assertion follows from the inductive hypothesis. Here we have proved also (3.16.15) in the case $v_{j}<\mu_{j}$ for some $j$ such that $\mu_{j}>0$. Therefore it remains to show (3.16.15) in the case $\mu_{i}=v_{i}$ for $i \neq k$, where $\mu_{k} \leq 0$ (in particular $j \neq k$ ). By the above remark after (3.16.15), it is enough to show the strict injectivity of $\left(M_{v}\right.$, $F) \rightarrow\left(M_{\mu}, F\right)$, but this follows from that of $\left(V_{\mu_{j}^{\prime}}^{(j)} M_{v}, F\right) \rightarrow\left(V_{\mu_{j}^{\prime}}^{(j)} M_{\mu}, F\right)$ and $\left(\mathrm{Gr}_{\mu_{J}}^{V^{(J)}} M_{v}, F\right) \rightarrow\left(\mathrm{Gr}_{\mu_{J}}^{V^{(j)}} M_{\mu}, F\right)$, because we can apply the same argument as above to $\left(M_{v}, F\right)$ by replacing $\mu_{k}$ with $v_{k}$.

3.17. Proposition. With the notation as above, let $(M, F) \in \operatorname{MF}\left(\mathscr{D}_{X}\right)_{\text {rncqu }}$ and $g$ $=x^{m}$. We set $(\tilde{M}, F):=\left(i_{g}\right)_{*}(M, F)=\left(M\left[\partial_{t}\right], F\right)$, and let $V$ be the filtration $V$ along $X \times\{0\}$. Then:

$$
t: F_{p} V_{\alpha} \tilde{M} \stackrel{\sim}{\longrightarrow} F_{p} V_{\alpha-1} \tilde{M} \quad \text { for } \alpha<0,
$$




$$
\begin{array}{lll}
(3.17 .2) & \partial_{t}: F_{p} \mathrm{Gr}_{\alpha}^{V} \tilde{M} \stackrel{\sim}{\longrightarrow} F_{p+1} \mathrm{Gr}_{\alpha+1}^{V} \tilde{M} & \text { for } \alpha>-1, \\
(3.17 .3) & F_{p} V_{\alpha} \tilde{M}=\sum\left(F_{p-i} V_{m \alpha} M \otimes 1\right) F_{i}\left(\mathscr{D}_{X}[s]\right) & \text { for } \alpha<0, \\
(3.17 .4) & \left(\mathrm{Gr}_{\alpha}^{V} \tilde{M}, F\right) \in \operatorname{MF}\left(\mathscr{D}_{X}\right)_{r n c q u} & \text { for } \alpha<0, \\
(3.17 .5) & F_{p} V_{v}\left(\mathrm{Gr}_{\alpha}^{V} \tilde{M}\right)=\sum \mathrm{Gr}_{\alpha}^{V}\left(F_{p-i} V_{v+\alpha m} M \otimes 1\right)(s-\alpha)^{i} \\
& & \text { for } v \in\left(\mathbf{Q}_{\leq 0}\right)^{n}, \alpha<0 .
\end{array}
$$

Moreover if one of the conditions:

$$
\begin{aligned}
& x_{i}: F_{p} \mathrm{Gr}_{v+1_{\imath}}^{V} M \stackrel{\sim}{\longrightarrow} F_{p} \mathrm{Gr}_{v}^{V} M \\
& \partial_{i}: F_{p} \mathrm{Gr}_{v}^{V} M \stackrel{\sim}{\longrightarrow} F_{p+1} \mathrm{Gr}_{v+1_{\imath}}^{V} M \\
& \partial_{i}: F_{p} \mathrm{Gr}_{v}^{V} M \longrightarrow F_{p+1} \mathrm{Gr}_{v+1_{\imath}}^{V} M
\end{aligned}
$$

for any $i \in \bar{m}\left(\right.$ i.e. $\left.m_{i} \neq 0\right), v \in(\mathbf{Q} \cap[-1,0])^{n}$ such that $v_{i}=-1$, is satisfied, we have respectively:

$$
\text { (3.17.1) and (3.17.3) hold also for } \alpha=0
$$

$$
\text { (3.17.2) holds also for } \alpha=-1
$$

$$
\left(F_{p} \operatorname{Gr}_{-1}^{V} \tilde{M}\right) \partial_{t}=F_{p+1} \operatorname{Gr}_{0}^{V} \tilde{M}
$$

Proof. Put $j:\{g \neq 0\} \rightarrow X$. Then (3.17.6) (resp. (3.17.7)) implies $M=j_{*}^{\text {reg }} j^{-1} M$ (resp. $j_{!}^{\text {reg }} j^{-1} M$ ), and (3.17.1) for $\alpha=0$ (resp.(3.17.10)) is reduced to (3.17.3) for $\alpha$ $=0$ (resp. (3.17.11)), because the above isomorphism implies the assertion forgetting $F$. In general, (3.17.1) follows from (3.17.3), and we get (3.17.2) (and (3.17.11) if (3.17.8) is satisfied) by the same argument as the proof of (3.5.2), using (3.16.8) (or (3.16.7)) and 3.4 (because (3.16.7) and (3.17.8) imply $F$ is generated by its restriction to $\left.\left(\cap_{i \in \bar{m}} V_{<0}^{(i)} M\right) \cap\left(\cap_{i \notin \bar{m}} V_{0}^{(i)} M\right)\right)$. We show (3.17.3) as follows.

Put $V_{m \alpha}^{\prime} M=\cap_{i \in \bar{m}} V_{m_{2} \alpha}^{(i)} M, \mathscr{D}^{\prime}=\mathcal{O}_{X}\left[\partial_{\bar{m}}\right]$, where $\partial_{\bar{m}}=\left\{\partial_{i}\right\}_{i \in \bar{m}}$.

We define the action of $\widetilde{\partial}_{i}$ on $M \otimes_{(i} \mathscr{D}^{\prime}[s]$ by

$$
(u \otimes P) \rightarrow u x_{i} \partial_{i} \otimes P-u \otimes x_{i} \partial_{i} P-u \otimes m_{i} s P .
$$

We define $\left(K_{\alpha} ; F, G\right)$ by the Koszul complex

$$
K\left(V_{m \alpha}^{\prime} M \otimes_{\mathcal{O}} \mathscr{D}^{\prime}[s] ; \tilde{\partial}_{i}(i \in \bar{m})\right)[|\bar{m}|],
$$

where $F, G$ on $K_{\alpha}^{0}=V_{m \alpha}^{\prime} M \otimes \mathscr{D}^{\prime}[s]$ are defined by

$$
\begin{aligned}
F_{p} K_{\alpha}^{0} & =\sum F_{p-i} V_{m \alpha}^{\prime} M \otimes F_{i}\left(\mathscr{D}^{\prime}[s]\right) \\
G_{j} K_{\alpha}^{0} & =V_{m \alpha}^{\prime} M \otimes F_{j}\left(\mathscr{D}^{\prime}[s]\right)
\end{aligned}
$$

and they are shifted by $j$ on $K_{\alpha}^{-j}$. By (3.4.5) we have the natural $\mathscr{D}_{X}[\mathrm{~s}]$-linear 
morphism: $H^{0}\left(K_{0}\right) \rightarrow \tilde{M}$ whose image is $V_{\alpha} \tilde{M}$ by 3.4. Therefore it is enough to show this morphism is strictly compatible with $F$ by (3.16.8). Let $G$ be the filtration on $\tilde{M}=M\left[\partial_{t}\right]$ by the degree of $\partial_{t}$. Then we have

$$
H^{0}\left(K_{\alpha} ; F, G\right) \longrightarrow(\tilde{M} ; F, G),
$$

and it is enough to show its strict injectivity. Because $\mathrm{Gr}^{F} \mathrm{Gr}^{G} K_{\alpha}$ is the Koszul complex of $\operatorname{Gr}^{F} V_{m \alpha}^{\prime} M\left[\xi_{\bar{m}}, s\right]$ with the action $-\left(x_{i} \xi_{i}+m_{i} s\right)$ for $i \in \bar{m}$, and $\mathrm{Gr}^{F} \mathrm{Gr}^{G}$ of (3.17.12) is given by $\xi_{i} \mapsto-\left(\partial_{i} g\right) \tau$, $s \mapsto g \tau$ (where $\operatorname{Gr}^{F} \mathrm{Gr}^{G} \tilde{M}=\operatorname{Gr}^{F} M[\tau]$ ), it is enough to show $\left\{x_{i} \xi_{i}+m_{i} s(i \in \bar{m}), g\right\}$ is a regular sequence of $\mathrm{Gr}^{F} V_{m \alpha}^{\prime} V_{v}^{\prime \prime} M\left[\xi_{\bar{m}}\right.$, $s]$ for $v \in \mathbb{Q}^{n \bar{m}}$ by the same argument as the proof of (3.5.1) (using the localization by $g$, and taking the limit $v \rightarrow+\infty)$. But it can be reduced to the flatness of $\mathrm{Gr}^{F} V_{m \alpha}^{\prime} V_{v}^{\prime \prime} M$ over $\mathbb{C}\left\{x_{\bar{m}}\right\}$, and we can verify this by induction on $v$ and on $\operatorname{dim} X$. If (3.17.6) is satisfied, the above argument applies also to $\alpha=0$, because $V_{0} \tilde{M}$ is generated by $V_{0} M \otimes 1$ in this case (i.e. $g$ (or $t$ ) acts bijectively on $M$ (or $\tilde{M})$ ). Therefore it remains to show (3.17.4-5).

Put $S_{v}=V_{v} M \otimes \mathbb{C}[s]$ for $v \in\left(\mathbb{Q}_{>0}\right)^{\bar{m}} \times\left(\mathbb{Q}_{\geq 0}\right)^{\bar{n} \bar{m}}$ and define the action of $x_{i} \partial_{i}$ by $\left(u \otimes s^{j}\right) x_{i} \partial_{i}=\left(u x_{i} \partial_{i} \otimes s^{j}\right)-\left(u \otimes m_{i} s^{j+1}\right)$. Then, for $\alpha<0$, we have the natural $\mathcal{O}_{X}[s]$-linear morphism:

$$
A_{\alpha}: S_{m \alpha} \longrightarrow \mathrm{Gr}_{\alpha}^{V} \tilde{M}
$$

compatible with the action of $x_{i} \partial_{i}$. By the proof of 3.4, we have $\operatorname{Im} A_{\alpha}$ $=V_{0}\left(\operatorname{Gr}_{\alpha}^{V} \tilde{M}\right)$. Put $S_{\alpha}=S_{m \alpha}, R_{\alpha}=\operatorname{Ker} A_{\alpha}$. We define $F, V^{(i)}$ on $S_{v}$ by

$$
F_{p} S_{v}=\sum F_{p-j} V_{v} M \otimes s^{j}, \quad V_{\beta}^{(i)} S_{v}=\left(V_{\beta+v_{v}}^{(i)} M \cap V_{v} M\right)[s]
$$

so that the $n+1$ filtrations $F, V^{(i)}$ on $S_{v}$ are compatible, and $A_{\alpha}$ preserves $F, V^{(i)}$ and is strictly compatible with $V^{(i)}$ for any $i$. We define $W$ on $S_{v}$ by

$$
W_{k} S_{v}=\sum_{I \subset I(v),|I| \leq k} V_{<0}^{I(v) \backslash I} S_{v}
$$

where $I(v)=\left\{i \in \bar{m}: \operatorname{Gr}_{v_{2}}^{V^{(i)}} M \neq 0\right\}$ and $V_{<0}^{I}=\bigcap_{i \in I} V_{<0}^{(i)} . \quad$ By induction on $n$ and $|I(v)|$, we show that the $n+2$ filtrations $F, W, V^{(i)}$ on $S_{v}$ are compatible and

$$
F_{p} W_{k} V_{\mu} S_{v}=\sum_{I \subset I(v),|I| \leq k} F_{p} V_{<0}^{I(v) \backslash I} V_{\mu} S_{v}
$$

using $[19,1.2 .12]$ and taking $\operatorname{Gr}_{0}^{V(t)} S_{v}$ for $i \in I(v)$. Then $\mathrm{Gr}_{k}^{W} S_{v}$ is the direct sum of $\mathrm{Gr}_{0}^{V^{I}} V_{<0}^{I(v) \backslash I} S_{v}$ for $I \subset I(v)$ such that $|I|=k$ and this decomposition is compatible with $F, V^{(i)}$. By $3.4, \mathrm{Gr}_{k}^{W} R_{\alpha}$ is the direct sum of the images of the endomorphisms $\prod_{i \in I} x_{i} \partial_{i}$ on these direct factors, where $v=m \alpha$ and the action of $x_{i} \partial_{i}$ is defined as above. Using the filtration by the degree of $s$, we verify:

$$
\prod_{i \in I} x_{i} \partial_{i}:\left(S_{\alpha} ; F, V^{(i)}\right) \longrightarrow\left(S_{\alpha} ; F[-|I|], V^{(i)}\right)
$$

are strictly injective as $n+1$ filtered morphisms. In particular, their restriction to $\mathrm{Gr}_{0}^{V^{I}} V_{<0}^{I(\alpha) \backslash I} S_{\alpha}$ are also strictly injective so that the $n+1$ filtrations on $\mathrm{Gr}_{k}^{W} R_{\alpha}$ 
are compatible. Moreover the $n+2$ filtrations $F, W, V^{(i)}$ on $R_{\alpha}$ are also compatible by $[19,1.2 .12]$, because $\left(\prod_{i \in I} x_{i} \partial_{i}\right)\left(V_{<0}^{I(\alpha) \backslash I} S_{\alpha}\right) \subset R_{\alpha}$ and $[19,1.2 .12$.vi] is satisfied. Then $R_{\alpha} \rightarrow S_{\alpha}$ is strictly injective as an $n+2$ filtered morphism (taking $\left.\mathrm{Gr}_{k}^{W}\right)$, and we define $F^{\prime}, W$ on $\operatorname{Im} A_{\alpha}=V_{0}\left(\mathrm{Gr}_{\alpha}^{V} \tilde{M}\right)$ to be the induced filtration by $F, W$ on $S_{\alpha}$ so that the $n+2$ filtrations $F^{\prime}, W, V^{(i)}$ are compatible. Then the conditions (3.16.9-11) are satisfied for $F^{\prime}, V^{(i)}$ on $V_{0}\left(\mathrm{Gr}_{\alpha}^{V} \tilde{M}\right)$, because (3.16.10) is satisfied taking $\mathrm{Gr}_{k}^{W}$ (and using the filtration by the degree of $s$ ), and (3.16.11) is verified using the strict surjectivity of $A_{\alpha}$. Then by (3.16.12) and (3.17.3), we get $F=F^{\prime}$ and (3.17.4-5). This completes the proof of 3.17 .

3.18. Let $X$ be a complex manifold of dimension $n$, and $D$ a reduced normal crossing divisor on $X$. Put $j: U=X \backslash D \rightarrow X$. Let $(M, F)$ be the underlying filtered $\mathscr{D}_{X}$-Module of a Hodge Module of weight $w$ with strict support $X$, and assume $\left.M\right|_{U}$ is coherent over $\mathcal{O}_{U}$, i.e.

$(L, F)=\left.(M, F) \otimes\left(\Omega_{X}^{n}, F\right)^{-1}\right|_{U}$ underlies a variation of Hodge structure of weight $w-n$.

Then we have the canonical isomorphism:

$$
(M, F) \cong\left(j_{!_{*}}\left(\Omega_{U}^{n} \otimes L\right), F\right)
$$

where the right hand side is defined as in (3.10.11). In fact, the assertion is wellknown forgetting $F$, and is local. Therefore we may assume the notations are as in 3.2. Then, by $3.12,\left(M^{\prime}, F\right)$ the right hand side of (3.18.2) satisfies:

$$
\begin{aligned}
& \left(M^{\prime}, F\right) \in M F\left(\mathscr{D}_{X}\right)_{r n c q u} \\
& \partial_{i}: \operatorname{Gr}_{v}^{V}\left(M^{\prime}, F\right) \rightarrow \mathrm{Gr}_{v+1_{i}}^{V}\left(M^{\prime}, F[-1]\right) \text { are strictly surjective for any } \\
& i \text { and } v \in(\mathbb{Q} \cap[-1,0])^{n} \text { such taht } v_{i}=-1 .
\end{aligned}
$$

Therefore by $3.17,\left(M^{\prime}, F\right)$ is quasi-unipotent and regular along any $g$ such that $g^{-1}(0)_{\text {red }}=D$, and (3.18.2) follows from [19,3.2.2]. We shall show later the converse in the polarizable case, cf. 3.20.

Now, with the notation of $3.2,3.4$, assume $(M, F) \in \mathrm{MF}\left(\mathscr{D}_{X}\right)_{\text {rncqu }}$ satisfies (3.18.1-2) and the variation of Hodge structure in (3.18.1) is polarized, where $(M$, $F$ ) is identified with a coherent filtered $\mathscr{D}$-Module on $X$ by restricting $X$. We define $\left\{\left(E_{I}^{v}, F\right)\right\}=\Psi_{\mathscr{P}}^{n}(M, F)$ by

$$
\left(E_{I}^{v}, F\right)=\mathrm{Gr}_{v+1_{I}}^{V}(M, F[n-|I|]),
$$

for $v \in(\mathbb{Q} \cap[-1,0))^{n}, I \subset \bar{n} \backslash \bar{v} . \quad$ Put

$$
\left(E_{I}^{J}, F\right)=\bigoplus_{\bar{v}=J}\left(E_{I}^{v}, F\right) .
$$

Then, by $[8,(1.16)][15,2.1 .5]$ and $(3.18 .4)$, we have:

(3.18.7) $\quad\left(\left(E_{I}^{J}, F\right), N_{1}, \ldots, N_{n}\right)$ underlies a nilpotent orbit of weight $w+|I|-n$, 
where its $\mathbb{Q}$-(or $\mathbb{R}-)$ structure is induced by $\Psi_{\mathscr{D}}^{n}=\Psi^{n} \circ \mathrm{DR}$, and the polarization by $\Psi_{(\mathscr{Q})}^{n} \circ \mathbb{D}=\mathbb{D} \circ \Psi_{(\mathscr{D})}^{n}$, cf. 3.2. In particular, $\left(E_{I}^{J} ; F, W\right)$ is a mixed Hodge structure, where $W$ is the monodromy filtration for $\sum_{i} N_{i}$ shifted by $w+|I|-n$.

Put $g=x^{m}$ as in 3.3. We define $\widetilde{E}_{I}^{v}$ by (3.3.1), and put $\widetilde{E}_{I}^{J}=\bigoplus_{|v|=J} \widetilde{E}_{I}^{v}$ so that $\widetilde{E}_{I}^{J}$ has the $\mathbb{R}$-structure induced by the natural one on $E_{I}^{J}[N]$, where we choose $i=\sqrt{-1}$ so that the Tate twist $\otimes \mathbb{Z}(n)$ is trivialized and $N$ is defined over $\mathbb{Q}$, i.e. $N=\log T_{u}$. We also define the filtration $F, W$ on $\widetilde{E}_{I}^{J}$ so that they are compatible with the decomposition $\tilde{E}_{I}^{J}=\oplus \tilde{E}_{I}^{v}$ and $F_{p} \tilde{E}_{I}^{v}, W_{k} \widetilde{E}_{I}^{v}$ are the quotients of

$$
\begin{array}{ll}
F_{p-1+b} E_{I \backslash \bar{m}}^{\mu}[N], W_{k+2-2 b} E_{I \backslash \bar{m}}^{\mu}[N] & \text { if } I \neq 0 \\
F_{p-1+b} E_{I^{\prime} \backslash \bar{m}}^{\mu}[N] \oplus F_{p} E_{I^{\prime}}^{\mu}[N], W_{k+2-2 b} E_{I^{\prime} \backslash \bar{m}}^{\mu}[N] \oplus W_{k} E_{I^{\prime}}^{\mu}[N] \text { otherwise }
\end{array}
$$

where $\mu=v^{\prime}+v_{0} m \in(\mathbb{Q} / \mathbb{Z})^{n}, I^{\prime}=I \backslash\{0\}, b=|I \cap \bar{m}|$ and

$$
F_{p} E_{I}^{\mu}[N]=\sum F_{p-j} E_{I}^{\mu} \otimes N^{j}, W_{k} E_{I}^{\mu}[N]=\sum W_{k+2 j} E_{I}^{\mu} \otimes N^{j} .
$$

Then $\left(\widetilde{E}_{I}^{\nu} ; F, W\right)$ is isomorphic to

$$
\begin{array}{ll}
\bigoplus_{0 \leq j<b}\left(E_{I \backslash \bar{m}}^{\mu} ; F, W\right)(1-b+j) & \text { if } I \neq 0, \\
\bigoplus_{0 \leq j<b-1}\left(E_{I^{\prime} \backslash \bar{m}}^{\mu} ; F, W\right)(1-b+j) \oplus\left(E_{I^{\prime}}^{\mu} ; F, W\right) & \text { otherwise, }
\end{array}
$$

forgetting the action of $\tilde{c a n}_{i}$, etc. Therefore $\left(\widetilde{E}_{I}^{J} ; F, W\right)$ are mixed Hodge structures. We also verify that $\tilde{\operatorname{can}}_{i}, \widetilde{\operatorname{Var}}_{i}$ and $\tilde{N}_{i}$ induce morphisms of mixed Hodge structures. In particular,

$$
\operatorname{can}:\left(\widetilde{E}_{I^{\prime}}^{v} ; F, W\right) \longrightarrow\left(\widetilde{E}_{I}^{v} ; F, W\right)
$$

is strictly surjective and $\left(\widetilde{E}_{I}^{v} ; F, W\right)$ is identified with

$$
\operatorname{Coim}\left(N:\left(\widetilde{E}_{I^{\prime}}^{v} ; F, W\right) \rightarrow\left(\widetilde{E}_{I^{\prime}}^{v} ; F, W\right)(-1)\right)
$$

if $I \ni 0$, because can : $\psi_{g, 1} \rightarrow \phi_{g, 1}$ is surjective. By 3.17 the isomorphism (3.4.2) is compatible with $F$, i.e.

$$
\left(\widetilde{E}_{I}^{v}, F\right)=\mathrm{Gr}_{v^{\prime}+1_{I}}^{V}\left(\mathrm{Gr}_{v_{0}+\left(1_{I}\right)_{0}}^{V}(M, F[n+1-|I|])\right),
$$

because the case $I \ni 0$ is reduced to the other case by (3.17.11) and the above argument, where we use the strictness of $N: \operatorname{Gr}_{-1}^{V}(\tilde{M}, F) \rightarrow \operatorname{Gr}_{-1}^{V}(\tilde{M}, F)(-1)$ in $\operatorname{MF}\left(\mathscr{D}_{X}\right)_{\text {rncqu }}$, cf. 3.13. Similarly $\left(\widetilde{E}_{I}^{J}, F\right)$ underlies a nilpotent orbit by the following result of Kashiwara, because the case $I \ni 0$ follows from the other case using the above strict surjectivity and $[8,1.16][15,2.1 .5]$.

3.19. Proposition. Let $\left((H ; F, W) ; N_{i}(1 \leq i \leq n) ; S\right)$ be a nilpotent orbit of weight $w$, where $W$ is the monodromy filtration for $\sum N_{i}$ shifted by $w$. Take $m \in \mathbb{N}^{n}, I \subset \bar{m}$ such that $I \neq \phi$, and put 


$$
\begin{aligned}
\left(\tilde{H}_{I} ; F, W\right) & =\operatorname{Coker}\left(\tilde{N}_{I}:(H[N] ; F[l], W[-2 l]) \rightarrow(H[N] ; F, W)\right) \\
( & \left.=\bigoplus_{0 \leq j<l}(H ; F, W)(j)\right)
\end{aligned}
$$

where $l=|I|, \tilde{N}_{I}=\prod_{i \in I} \tilde{N}_{i}, \tilde{N}_{i}=N_{i}-m_{i} N$ and

$$
F^{p}(H[N])=\sum_{j} F^{p+j} H \otimes N^{j}, W_{k}(H[N])=\sum_{j} W_{k+2 j} H \otimes N^{j} .
$$

Then $\left(\left(\widetilde{H}_{I} ; F, W\right) ; N, \widetilde{N}_{i}+m_{i} N(1 \leq i \leq n) ; \widetilde{S}_{I}\right)$ is a nilpotent orbit of weight $w$ $+1-l ;$ in particular $W$ is the monodromy filtration for $N+\sum\left(\tilde{N}_{i}+m_{i} N\right)$ shifted by $w+1-l$. Here $\widetilde{S}_{I}$ is defined by

$$
\tilde{S}_{I}(\tilde{u}, \tilde{v})=\operatorname{Res} S\left(\tilde{N}_{I}^{-1} \tilde{u}, \tilde{v}\right) \quad \text { for } \tilde{u}, \tilde{v} \in \tilde{H}_{I},
$$

where $S$ is extended to $S: H\left[N, N^{-1}\right] \otimes H\left[N, N^{-1}\right] \rightarrow \mathbf{C}\left[N, N^{-1}\right]$ by $S\left(u \otimes N^{i}, v \otimes N^{j}\right)=(-1)^{i} S(u, v) \otimes N^{i+j}$ and $\operatorname{Res}\left(\sum a_{i} \otimes N^{i}\right)=a_{-1}$.

(See Appendix for the proof.)

Remark. We use Deligne's convention of positivity (i.e. $S(u, C \bar{u}) \geq 0$ ) so that we have

$$
\tilde{S}_{I}\left(\tilde{\operatorname{can}}_{i} \tilde{u}, \tilde{v}\right)=\tilde{S}_{I^{\prime}}\left(\tilde{u}, \tilde{\operatorname{Var}}_{i} \tilde{v}\right)
$$

for $\tilde{u} \in \tilde{H}_{I^{\prime}}, \tilde{v} \in \tilde{H}_{I}$, where $I=I^{\prime} \sqcup\{i\} \subset \bar{m}$ and $\tilde{c a n}_{i}\left(\right.$ resp. $\left.\widetilde{V a r}_{i}\right)$ is induced by $N_{i}$ $-m_{i} N$ (resp. id) on $H[N]$, cf. 3.3. In the case $(H, F)=\left(E_{\phi}^{\phi}, F\right)$, where $\left\{\left(E_{I}^{J}, F\right)\right\}$ corresponds to $(M, F)$ as in 3.18 , we can verify that $\widetilde{S}_{I}$ corresponds to the duality on $\psi_{g}(M, F)$, cf. [25]. Here the assertion for the $\mathbf{R}$-part is essentially due to Kashiwara.

From the above results, we can deduce:

3.20. Theorem. Let $X$ be a complex manifold of dimension $n, D$ a reduced normal crossing divisor, and $j: U=X \backslash D \rightarrow X$ the natural inclusion. Let $(M, F$, $K)$ be a filtered regular holonomic $\mathscr{D}_{X}$-Module with $\mathbf{Q}$ - (or $\mathbf{R}$-) structure such that (3.18.1-2) are satisfied and the variation of Hodge structure in (3.18.1) is polarized by $S^{\prime}$. Then $(M, F, K)$ is a Hodge Module of weight $w$ polarized by the duality $S$ induced by $S^{\prime}$.

Proof. By (3.15.2), $S^{\prime}$ induces the duality $S$ of $(M, F, K)$. We show by induction $n$ that $(M, F, K, S)$ is a polarized Hodge Module. The assertion is clear if $n=0$. For $n>0$, we have to verify the condition in $[19,5.1 .8]$ for $\psi_{g}$ and $[19,(5.2 .10 .2)]$, because $[19,5.1 .8]$ for $\phi_{g, 1}$ follows from $[19,5.1 .17]$ and $(M$, $F$ ) is quasi-unipotent and regular along $g$ by 3.17. Therefore the assertions are local. We may assume $g^{-1}(0)_{\text {red }} \cup D$ is a normal crossing divisor by Hironaka's desingularization and using $[19,5.3 .4]$. Then we may assume $g^{-1}(0)_{\text {red }} \subset D$ by replacing $D$ with $g^{-1}(0)_{\text {red }} \cup D$, because $\left(j_{! *} j^{-1} M, F\right)$ is uniquely characterized by the condition (3.18.1) and 
at any $x \in X$, there exists a coordinate neighborhood $\Delta^{n}$ of $x$ such that $(M, F)_{x} \in \operatorname{MF}\left(\mathscr{D}_{\Delta^{n}}\right)_{r n c q u}$ (cf. 3.16) and (3.18.4) is satisfied for $(M, F)_{x}$.

Taking a finite covering (and by $[19,5.3 .4]$ ), we may assume further that $M$ has unipotent monodromies and $g=\left(x_{1} \cdots x_{l}\right)^{a}$ with local coordinates $\left(x_{1}, \cdots, x_{n}\right)$. By inductive hypothesis, it is enough to show:

$W$ on $\psi_{g}(M, F)$ is defined in $\operatorname{MF}\left(\mathscr{D}_{\Delta^{n}}\right)_{\text {rncqu }}$ at each point of $X$ and the primitive decomposition of $\operatorname{Gr}_{k}^{W} \psi_{g}(M, F)$ is compatible with $F$,

$P \mathrm{Gr}_{k}^{W} \psi_{g}(M, F, K)$ admits the strict support decomposition compatible with $F$ such that each $Z$-component corresponds generically to a polarized variation of Hodge structure of weight $k-\operatorname{dim} Z$ and its pull-back to $Z$ satisfies (3.20.1) at any point of $Z$,

where $W$ is the monodromy filtration shifted by $w-1$, and $Z$ in (3.20.3) are smooth (because the assertion is local). Here note that $(M, F) \in \operatorname{MF}\left(\mathscr{D}_{\Delta^{n}}\right)_{r n c q u}$ is of the form $i_{*}\left(M^{\prime}, F\right)$ with $i: \Delta^{m} \rightarrow \Delta^{n}$ and $\left(M^{\prime}, F\right) \in \operatorname{MF}\left(\mathscr{D}_{\Delta^{m}}\right)_{r n c q u}$, if $\operatorname{supp} M$ $\subset \Delta^{m}$ for $m<n$.

We first get (3.20.2) by 3.13 and Deligne's inductive construction of $W[10]$, because $N$ on $\tilde{E}_{I}^{J}$ underlies a morphism of mixed Hodge structures. For the strict support decomposition in (3.20.3), it is enough to show:

$$
\begin{aligned}
P \mathrm{Gr}_{k}^{W} \widetilde{E}_{I}^{J}= & \operatorname{In}\left(\tilde{\operatorname{can}}_{i}: P \mathrm{Gr}_{k}^{W} \widetilde{E}_{I^{\prime}}^{J} \longrightarrow P \mathrm{Gr}_{k}^{W} \widetilde{E}_{I}^{J}\right) \\
& \oplus \operatorname{Ker}\left(\widetilde{\operatorname{Var}}_{i}: P \mathrm{Gr}_{k}^{W} \widetilde{E}_{I}^{J} \longrightarrow P \mathrm{Gr}_{k}^{W} \widetilde{E}_{I^{\prime}}\right)
\end{aligned}
$$

for any $I, I^{\prime} \subset \bar{n}, i \in \bar{n}$ such that $I=I^{\prime} \sqcup\{i\}$, where $\widetilde{E}_{I}^{J}=0$ for $J \neq \phi,\{0\}$ and $\widetilde{E}_{I}^{\{0\}}=\left(\widetilde{E}^{\phi}\right)^{a-1}$. In fact, we can use $[19,5.1 .4]$ inductively, combined with the equivalence of categories (3.1.2)(3.2.2), because the condition (3.20.4) is stable by direct factor and induces a direct sum decomposition in the category of mixed Hodge structures so that $\widetilde{c a n}_{i}, \widetilde{\operatorname{Var}}_{i}$ induce strict morphisms in $\operatorname{MF}\left(\mathscr{D}_{\Delta^{n-1}}\right)_{\text {rncqu }}$ by 3.13. Here we have also proved the condition (3.20.1) for any $Z$ component. To show (3.20.4) we have to use 3.19. Here we may assume $J=\phi$ in (3.20.4). Then we have

$$
\tilde{E}_{I}^{\phi}=\operatorname{Coker}\left(\tilde{N}_{I \cap \bar{m}}: E_{\backslash \backslash \bar{m}}^{\phi}[N] \longrightarrow E_{\backslash \bar{m}}^{\phi}[N]\right)
$$

by definition. If we define $\tilde{S}_{I}$ on $E_{I}^{\phi}$ as in 3.19 (where we replace $\tilde{N}_{I}$ by $\tilde{N}_{I \cap \bar{m}}$ in (3.19.1)), we get (3.19.2) for any $I, I^{\prime} \subset \bar{n}, i \in \bar{n}$ such that $I=I^{\prime} \sqcup\{i\}$. Therefore the assertion follows from $[19,5.2 .15]$ and the remark below, because $\tilde{N}_{i}=N_{i}$ on $\mathrm{Gr}^{W}$. Here we restrict to the nilpotent orbit of two variables for $N, N_{i}$ (by changing $F$ ). Then we first take the limit for $N$ and $P \mathrm{Gr}_{k}^{W}$ to get a nilpotent orbit for $N_{i}$, and then take the limit for $N_{i}$ and $\mathrm{Gr}^{W^{(i)}}$, where $W^{(i)}$ is the monodromy filtration for $N_{i}$. Now it remains to show that each $Z$-componemt 
of $\mathrm{PGr}_{k}^{W} \psi_{g}(M, F, K)$ corresponds generically to a polarized variation of Hodge structure of weight $k-\operatorname{dim} Z$. But this follows from 3.19 and its remark, because at the generic point of $Z$, the $Z$-component of $\operatorname{PGr}_{k}^{W} \psi_{g}(M, F, K)$ corresponds to a direct factor of $P \mathrm{Gr}_{k-w+1}^{W^{\prime}} \widetilde{E} \phi$ on which the action of $N_{i}$ is trivial, where $Z=D_{I}$ and $W^{\prime}$ on $\tilde{E}_{T}^{\phi}$ is the monodromy filtration (not shifted) so that $P \mathrm{Gr}_{k-w+1}^{W^{\prime}} \widetilde{E}_{I}^{\phi}$ underlies a nilpotent orbit of weight $k+|I|-n=k-\operatorname{dim} Z$, cf. 3.18. This completes the proof of 3.20 .

Remark (Kashiwara). Let $(H, F, N, S)$ and $\left(H^{\prime}, F, N, S^{\prime}\right)$ be nilpotent orbits of one variable of weight $w$ and $w+1$, and $u:(H, F) \rightarrow\left(H^{\prime}, F\right), v:\left(H^{\prime}, F\right) \rightarrow(H, F[$ -1]) morphisms defined over $\mathbb{R}$ such that $v u=N, u v=N$ and

$$
S^{\prime}(u x, y)= \pm S(x, v y) \text { for } x \in H, y \in H^{\prime}
$$

Then $\mathrm{Gr}^{W} u=0, \mathrm{Gr}^{W} v=0$, i.e. $u\left(W_{k} H\right) \subset W_{k-1} H^{\prime}, v\left(W_{k} H^{\prime}\right) \subset W_{k-1} H$, where $W$ is the monodromy filtration. In fact, we can reduce to the case $N=0$ using the primitive decomposition, then to the case $\operatorname{dim} H=1$ or 2 (same for $H^{\prime}$ ).

As a corollary we get (by [19, 3.2.2 and 5.3.1]):

3.21. Theorem. For a reduced irreducible separated complex analytic space $X$ of dimension $n$, we have an equivalence of categories:

$$
\mathrm{MH}_{X}(X, w)^{p} \stackrel{\sim}{\longrightarrow} \operatorname{VHS}_{\mathrm{gen}}(X, w-n)^{p}
$$

where $\mathrm{MH}_{X}(X, w)^{p}$ is the category of polarizable Hodge Modules of weight $w$ with strict support $X(c f .2 .1$ and $[19,5.1 .6])$ and $\operatorname{VHS}_{\mathrm{gen}}(X, w)^{p}$ is the inductive limit of $\operatorname{VHS}(U, w)^{p}$ the categories of polarizable variations of Hodge structures of weight $w$ with quasi-unipotent local monodromies on smooth dense Zariski open subsets $U$. Moreover the polarizations correspond bijectively.

Remark. 1) This implies the condition (2.7.6) in 2.7 , if $\mathscr{M}^{\prime}$ is polarizable and (2.7.1) is satisfied.

2) Let $f: X \rightarrow Y$ be a proper morphism of separated reduced complex analytic spaces such that $X$ is Kähler (or of class $\mathscr{C}$ in the sense of Fujiki). In this remark, we assume that the Hodge Modules are defined over $\mathbf{R}$, i.e. so are their underlying perverse sheaves. Then we have cohomological functors (cf. 2.13):

$$
\mathscr{H}^{j} f_{*}: \operatorname{MH}(X, w)^{p} \rightarrow \mathrm{MH}(Y, w+j)^{p} .
$$

In particular, Kollár's torsion-freeness holds also in the proper Kähler case, and the conjecture in the intorduction of [19] is verified.

To show $\mathscr{H}^{j} f_{*} \mathscr{M} \in \mathrm{MH}(Y, w+j)$, we may assume that $\mathscr{M} \in \mathrm{MH}_{X}(X, n)^{p}$ and $X$ is irreducible. Let $D$ be a closed subspace of $X$ such that $\mathscr{M}$ is a 
variation of Hodge structure on $X \backslash D$. For any $y \in Y$, we take $g_{1}, \ldots, g_{r} \in \mathcal{O}_{Y, y}$ such that $\cap g_{i}^{-1}(0)=\{y\}$, and take a resolution $\pi: \tilde{X} \rightarrow X$ such that $\pi^{-1}\left(D^{\prime}\right)$ is a normal crossing divisor, where $D^{\prime}=\cup g_{i}^{-1}(0) \cup D$. Let $\tilde{\mathscr{M}}$ be the polarizable Hodge Module with strict support $\tilde{X}$ whose restriction to $\tilde{X} \backslash \pi^{-1}\left(D^{\prime}\right)$ is isomorphic to that of $\mathscr{M}$ to $X \backslash D^{\prime}$. Then we can prove the assertions of [19, 5.3.1] for $\tilde{\mathscr{M}}$ and $\tilde{f}=f \pi: \tilde{X} \rightarrow Y$ on a neighborhood of $y$, using $[15,0.11 .1][16]$ and 3.20. In fact we get the hard Lefschetz for $\tilde{f}$ by the same argument as the proof of $[19,5.3 .1]$ using the inductive assumption, and we take further a resolution of $\tilde{X}$ associated to any point near $y$ to show the other assertions. We use $[19,5.3 .1]$ and Deligne's uniqueness of the decomposition for $\pi_{*} \tilde{\mathscr{M}}$ to deduce the assertion $\mathscr{H}^{j} f_{*} \mathscr{M} \in \mathrm{MH}(Y, w+j)$. For the global polarizability, we use the spectral sequence:

$$
E_{2}^{p q}=\mathscr{H}^{p} i^{*} \mathscr{H}^{q} f_{*} \mathscr{M} \Longrightarrow \mathscr{H}^{p+q+1} f_{*} \mathscr{H}^{-1} i^{*} \mathscr{M} \text { in } \operatorname{MHW}(Y)
$$

for a closed immersion $i: Y_{0} \rightarrow \mathrm{Y}$ such that $x_{0}:=f^{-1}\left(Y_{0}\right)$ is a locally principal divisor of $x$, where the natural inclusion $X_{0} \rightarrow X$ is also denoted by $i$, cf. [26].

\section{(3.c) Mixed Case}

3.22. Let $\mathrm{MH}$ be the abelian category of graded polarizable $\mathbb{Q}$-(or $\mathbb{R}$-) mixed Hodge structures $M=(H, F, W)$, where $H$ is the underlying $\mathbb{Q}$-(resp. $\mathbb{R}$-) module. Let MHL be the category of the objects of $\mathrm{MH}$ with a finite increasing filtration $L$. Let $\mathrm{MHL}^{n}$ be the category of the objects $(M, L)=(H, F, W, L)$ of MHL endowed with commuting $n$ morphisms

$$
N_{i}:(M, L) \rightarrow(M(-1), L) \quad(1 \leq i \leq n)
$$

such that $\left(\mathrm{Gr}_{k}^{L} H, F ; N_{i}(1 \leq i \leq n)\right)$ underlies a nilpotent orbit of weight $k$ and $W$ is the relative monodromy filtration of $(H, L)$ for $\sum_{1 \leq i \leq n} N_{i}$. Here $\mathrm{MHL}^{0}$ $=\mathrm{MH}$, i.e. $L=W$ on $H$, if $n=0$. Then we have:

(3.22.1) $\mathrm{MHL}^{n}$ is an abelian category such that any morphism induces a 3-filtered strict morphism for $F, W, L$.

In fact, the assertion is clear, if $M$ is pure (i.e. $L$ is trivial). In general it follows from $[19,5.1 .15]$. We have also

Assume the relative monodromy filtration $W^{(n)}$ of $(H, L)$ for $N_{n}$ exists, where $\left(M, L ; N_{i}\right)=\left((H, F, W), L ; N_{i}\right) \in \mathrm{MHL}^{n}$. Then $W^{(n)}$ induces the relative monodromy filtration of $(M, L)$ for $N_{n}$ in the exact category MHL (in particular, $F, W, W^{(n)}, L$ on $H_{\mathrm{C}}$ are compatible) and $\left(M, W^{(n)} ; N_{i}(1 \leq i \leq n-1)\right) \in \mathrm{MHL}^{n-1}$ so that $N_{n}:\left(M, W^{(n)}\right) \rightarrow\left(M(-1), W^{(n)}[2]\right)$ induces a morphism in $\mathrm{MHL}^{n-1}$. 
In fact the assertion follows from $[6, \S 3]$ in the pure case, because $N_{n}$ is a morphism in $\mathrm{MH}$ and $W^{(n)}$ is a filtration of $M$ in $\mathrm{MH}$. In general we can proceed by induction on the length of $L$ as in the proof of 2.4 using 1.2, 1.4-5 and (3.22.1). By the same argument as the proof of 2.5, we have:

(3.22.3) The assumption of (3.22.2) is stable by subquotients in $\mathrm{MHL}^{n}$. Moreover, for an exact sequence in $\mathrm{MHL}^{n}$ :

$$
0 \longrightarrow\left(M^{\prime}, L\right) \longrightarrow(M, L) \longrightarrow\left(M^{\prime \prime}, L\right) \longrightarrow 0
$$

such that $W^{(n)}$ exists on $(M, L)$, we have the exact sequence in $\mathrm{MHL}^{n-1}$ :

$$
0 \longrightarrow\left(M^{\prime}, W^{(n)}\right) \longrightarrow\left(M, W^{(n)}\right) \longrightarrow\left(M^{\prime \prime}, W^{(n)}\right) \longrightarrow 0 .
$$

Here we can use the splitting by the polarization on $\mathrm{Gr}_{k}^{L}$ of the exact sequence. We define the full subcategory $\mathrm{MHL}_{a d}^{n}$ of $\mathrm{MHL}^{n}$ by induction on $n: \mathrm{MHL}_{a d}^{0}=\mathrm{MHL}^{0}(\simeq \mathrm{MH})$ if $n=0$, and for $(M, L) \in \mathrm{MHL}^{n}(n>0)$, it belongs to $M H L_{a d}^{n}$ iff the assumption of (3.22.2) is satisfied and $\left(M, W^{(n)}\right) \in M H L_{a d}^{n-1}$. By definition, $\mathrm{MHL}_{a d}^{1}=\mathrm{MHL}^{1}$ and we have for $n \geq 2$ :

(3.22.4) for $\left((H, F, W), L ; N_{i}\right) \in \mathrm{MHL}^{n}$, it belongs to $\mathrm{MHL}_{a d}^{n}$ iff the relative monodromy filtration $W^{(i)}$ of $\left(H, W^{(i+1)}\right)$ for $N_{i}$ exists inductively for $1 \leq i \leq \mathrm{n}$, where $W^{(n+1)}:=L$.

By (3.22.3) we have:

(3.22.5) $\mathrm{MHL}_{a d}^{n}$ is stable by subquotients in $\mathrm{MHL}^{n}$ and for $((H, F, W)$, $\left.L ; N_{i}\right) \in \mathrm{MHL}_{a d}^{n}$ the induced filtration $W^{(i)}$ on $L_{k} H / L_{j} H$ coincides with the filtration $W^{(i)}$ defined inductively on $L_{k} H / L_{j} H$ by (3.22.3-4) for $k>j$.

Then $[6, \S 3]$ implies for $1 \leq i<j \leq n+1$ :

$$
\begin{aligned}
& W^{(i)} \text { is the relative monodromy filtration of }\left(H, W^{(j)}\right) \text { for any } \\
& \sum_{i \leq k<j} t_{k} N_{k} \text { such that } t_{k}>0 \text {. }
\end{aligned}
$$

In fact it is enough to show $N_{j} W_{k}^{(i)} \subset W_{k-2}^{(i)}$ for $i \leq j$. But it is clear if $i=j$, and follows from the functoriality of $W^{(i)}$ if $i<j$. By induction on $n$ and the length of $L$, we can also prove: $n+2$ filtrations $F, W^{(i)}(1 \leq i \leq n+1)$ on $H_{\mathrm{C}}$ are compatible,

(3.22.8) $\quad W^{(n)}$ induces the relative monodromy filtration of the $(n+1)$-filtered module $\left(H_{\mathrm{C}} ; F, W^{(i)}(1 \leq i<n), L\right)$ for $N_{n}:\left(H_{\mathbf{C}}, F, W^{(i)}, L\right) \rightarrow\left(H_{\mathbf{C}^{\prime}}\right.$ $\left.F[-1], W^{(i)}[2], L\right)$, 
any morphism in $\mathrm{MHL}_{a d}^{n}$ induces an $n+2$ filtered strict morphism of $\left(H_{\mathbf{C}}, F, W^{(i)}(1 \leq i \leq n+1)\right)$, where $W^{(n+1)}=L$, cf. (3.22.4).

In fact, in the pure case, (3.22.7) and (3.22.9) follow from the inductive hypothesis (because $\left.\left(M, W^{(n)}\right) \in \mathrm{MHL}_{\text {ad }}^{n-1}\right)$, and (3.22.8) from [6, §3]. In general (3.22.8) implies (3.22.7) inductively, and (3.22.9) is reduced to the pure case by (3.22.7) and $[19,5.1 .15]$. We prove (3.22.8) by induction on the length of $L$ using 1.2, (3.22.5) and (3.22.7), (3.22.9) for $\mathrm{MHL}_{a d}^{n-1}$.

3.23. Let $\mathbb{P}(n)_{u}$ be the full subcategory of $\mathbb{P}(n)$ (cf. 3.1) such that $E_{I}^{v}=0$ for $v \neq 0^{n} \in(\mathbb{C} / \mathbb{Z})^{n}$, i.e. the objects of $\mathbf{P}(n)$ are $\left\{E_{I}\right\}$ with morphisms

$$
\operatorname{can}_{i}: E_{I} \rightarrow E_{I^{\prime}}, \operatorname{Var}_{i}: E_{I^{\prime}} \longrightarrow E_{I}
$$

for $I^{\prime}=I \sqcup\{i\}$ such that $N_{i}=\operatorname{Var}_{i}^{\circ} \operatorname{can}_{i}$ or $\operatorname{can}_{i} \circ \operatorname{Var}_{i}$ are nilpotent and $\operatorname{can}_{i}$, $\operatorname{Var}_{j}$ commute mutually.

We define $\mathbb{M}(n)$ to be the category whose objects are $\mathscr{M}=\left\{M_{I}\right\}$ with morphisms $\operatorname{can}_{i}, \operatorname{Var}_{i}$ as above such that $M_{I}=\left\{H_{I}, F, W\right\} \in \mathbb{M H}$ (cf. 3.22) and $\operatorname{can}_{i}: M_{I} \rightarrow M_{I^{\prime}}, \operatorname{Var}_{i}: M_{I^{\prime}} \rightarrow M_{I}(-1)$ are morphisms of $\mathrm{MH}$. Then we have a natural functor $\mathbf{M}(n) \rightarrow \mathbb{P}(n)_{u}$. Note that $\mathbf{M}(n)$ is an abelian category by [9], and it has an inductive structure, i.e. $\mathbf{M}(n)$ is equivalent to the category whose objects are $\left(\mathscr{M}^{\prime} \rightleftarrows \mathscr{M}^{\prime \prime}\right)$ where $\mathscr{M}^{\prime}, \mathscr{M}^{\prime \prime} \in \mathbf{M}(n-1)$ and $\mathscr{M}^{\prime} \rightarrow \mathscr{M}^{\prime \prime}, \mathscr{M}^{\prime \prime} \rightarrow \mathscr{M}^{\prime}(-1)$ are morphisms in $\mathbf{M}(n-1)$. In fact, we associate $\left(\mathscr{M}^{\prime} \rightleftarrows \mathscr{M}^{\prime \prime}\right)$ to $\mathscr{M}$ so that

$$
M_{I}^{\prime}=M_{I}, M_{I}^{\prime \prime}=M_{I \cup\{n\}} \quad \text { for } I \subset \bar{n} \backslash\{n\} .
$$

In this case we also put:

$$
\psi_{(n)} \mathscr{M}=\mathscr{M}^{\prime}, \phi_{(n)} \mathscr{M}=\mathscr{M}^{\prime \prime} .
$$

We say $\mathscr{M} \in \mathbf{M}(n)$ has strict support $J\left(\right.$ or $\left.D_{J}\right)$, if $M_{I}=0$ for $I \not \supset J$ and $\operatorname{can}_{i}: M_{I}$ $\rightarrow M_{I^{\prime}}\left(\right.$ resp. $\operatorname{Var}_{i}: M_{I^{\prime}} \rightarrow M_{I}(-1)$ ) are surjective (resp. injective) for $I, I^{\prime} \supset J$ such that $I^{\prime}=I \sqcup\{i\}$. We say $\mathscr{M} \in \mathbf{M}(n)$ admits strict support decomposition, if we have a (unique) decomposition:

$$
\mathscr{M}=\oplus \mathscr{M}(J)
$$

such that $\mathscr{M}(J)$ has strict support $J$. In this case, $\mathscr{M}(J)$ is called the $J$-(or $D_{J^{-}}$) component of $\mathscr{M}$. Note that (3.23.2) is equivalent to

$$
\begin{aligned}
& M_{I}=\operatorname{Im}\left(\operatorname{can}_{i}: M_{I^{\prime}} \rightarrow M_{I}\right) \oplus \operatorname{Ker}\left(\operatorname{Var}_{i}: M_{I} \rightarrow M_{I^{\prime}}(-1)\right) \\
& \text { for } I=I^{\prime} \sqcup\{i\} .
\end{aligned}
$$

In fact we can verify this by induction on $n$ using the inductive structure of $\mathbb{M}(n)$ as above, because (3.23.3) is stable by direct factors in $\mathbf{M}(n)$ and the first factor of the right hand side of (2.23.3) is identified with Coim $N_{n}$ for $i=n$. We say $\mathscr{M} \in \mathbf{M}(n)$ is pure of weight $w$, if 
(3.23.4) $\mathscr{M}$ admits the strict support decomposition,

(3.23.5) $\quad\left(\left(H_{I}, F\right), \quad N_{i}(1 \leq i \leq n)\right)$ underlies a nilpotent orbit of weight $w-n+|I|$ such that $W$ is the monodromy filtration for $\sum_{1 \leq i \leq n} N_{i}$ shifted by $w-n+|I|$,

where $\mathscr{M}=\left\{M_{I}\right\}$ and $M_{I}=\left(H_{I}, F, W\right)$. (If $n=0, \mathscr{M}$ is a polarizable Hodge structure of weight $k$; in particular $\mathrm{Gr}_{i}^{W}=0$ for $i \neq k$.) Let $\mathscr{M}_{i}$ be pure of weight $k_{i}$ with strict support $J_{i}(i=1,2)$. Then

$$
\operatorname{Hom}\left(\mathscr{M}_{1}, \mathscr{M}_{2}\right)=0 \text { if } J_{1} \neq J_{2} \text { or } k_{1}>k_{2} \text {. }
$$

Let $\mathrm{ML}(n)$ be the category of the objects of $\mathbf{M}(n)$ with a finite increasing filtration $L$ such that $\operatorname{Gr}_{k}^{L}$ are pure of weight $k$. Then for $(\mathscr{M}, L)=\left\{\left(M_{I}\right.\right.$, $L)\} \in \mathbb{M L}(n)$, we have $\left(M_{I}, L[|I|-n] ; N_{i}\right) \in \operatorname{MHL}^{n}$ (cf. 3.22) by definition. We define the full subcategory $\mathbf{M L}(n)_{a d}$ of $\mathbf{M L}(n)$ by the condition:

$$
\left(M_{I}, L[|I|-n] ; N_{i}\right) \in \mathrm{MHL}_{a d}^{n} \text { for any } I .
$$

By [19, 5.1.15], (3.23.6), (3.22.5) and (3.22.9) we have

(3.23.8) $\quad \mathbf{M L}(n)$ is an abelian category such that any morphism induces 3 filtered strict morphisms on $\left(H_{I} \otimes \mathbb{C} ; F, W, L\right)$,

$$
\begin{aligned}
& \mathrm{ML}(n)_{a d} \text { is an abelian full subcategory of } \mathbf{M L}(n) \text { such that } \mathrm{ML}(n)_{a d} \\
& \text { is stable by subquotients in } \mathbf{M L}(n) \text { and any morphism induces } \\
& (n+2) \text {-filtered strict morphisms on }\left(H_{I} \otimes \mathbf{C} ; F, W^{(i)}[|I|-n]\right. \\
& (1 \leq i \leq n+1)) \text {, }
\end{aligned}
$$

where $W^{(n+1)}=L$ and $W^{(i)}$ is the relative monodromy filtration of $\left(H_{I}, W^{(i+1)}\right)$ for $N_{i}(1 \leq i \leq n)$ so that $W^{(1)}[|I|-n]=W$ by (3.22.6). By 1.5 and the same argument as in the proof of (3.20.4), we get the functors $\psi_{(n)}, \phi_{(n)}: \mathbf{M L}(n)_{a d}$ $\rightarrow \mathbb{M L}(n-1)_{a d}$ such that

$$
\begin{aligned}
& \psi_{(n)}(\mathscr{M}, L)=\left(\psi_{(n)} \mathscr{M}, W^{(n)}[-1]\right) \\
& \phi_{(n)}(\mathscr{M}, L)=\left(\phi_{(n)} \mathscr{M}, W^{(n)}\right),
\end{aligned}
$$

cf.(3.23.1). Then we have the natural morphisms:

$$
\begin{aligned}
& \operatorname{can}_{n}: \psi_{(n)}(\mathscr{M}, L) \longrightarrow \phi_{(n)}(\mathscr{M}, L) \\
& \operatorname{Var}_{n}: \phi_{(n)}(\mathscr{M}, L) \longrightarrow \psi_{(n)}(\mathscr{M}, L)(-1)
\end{aligned}
$$

by (3.23.3) for $i=n$ and 1.7. Here we define the Tate twist $(k)$ by

$$
(\mathscr{M}, L)(k)=(\mathscr{M}(k), L[-2 k]), \text { cf. }(2.17 .7) .
$$

Let $\mathbf{M L}(n)_{a d}^{n+1}$ be the category whose objects are $\mathscr{M}=\left\{M_{I}\right\} \in \mathbf{M}(n)$ with a finite 
increasing filtration $L$ and an endomorphism $N_{n+1}:(\mathscr{M}, L) \rightarrow(\mathscr{M}(-1), L)$ such that $\left(M_{I}, L[|I|-n-1] ; N_{i}\right) \in \mathrm{MHL}_{a d}^{n+1}$ for any $I$ and (3.23.3) holds for $\mathrm{Gr}_{k}^{L} \mathscr{M}$. Then $\operatorname{ML}(n)_{a d}^{n+1}$ is also an abelian category and we have a functor

$$
\psi_{(n+1)}: \operatorname{ML}(n)_{a d}^{n+1} \longrightarrow \mathbf{M L}(n)_{a d}
$$

by $\psi_{(n+1)}(\mathscr{M}, L)=\left(\mathscr{M}, W^{(n+1)}[-1]\right)$, where $W^{(n+1)}$ is the relative monodromy filtration of $(\mathscr{M}, L)$ for $N_{n}$. Let $\mathbf{M L}(n)_{a d}^{\prime}$ be the category whose objects are $\left\{\left(\mathscr{M}^{\prime}\right.\right.$, $\left.L),\left(\mathscr{M}^{\prime \prime}, L\right) ; u, v\right\}$ where $\left(\mathscr{M}^{\prime}, L\right) \in \mathbf{M L}(n-1)_{a d}^{n},\left(\mathscr{M}^{\prime \prime}, L\right) \in \mathbf{M L}(n-1)_{a d}$, and $u: \psi_{(n)}\left(\mathscr{M}^{\prime}, L\right) \rightarrow\left(\mathscr{M}^{\prime \prime}, L\right), v:\left(\mathscr{M}^{\prime \prime}, L\right) \rightarrow \psi_{(n)}\left(\mathscr{M}^{\prime}, L\right)(-1)$ are morphisms in $\mathbf{M L}(n$ $-1)_{a d}$ such that $v u=N_{n}$. Then by 1.9 we have an equivalence of categories

$$
\mathbf{M L}(n)_{a d} \stackrel{\sim}{\longrightarrow} \mathbf{M L}(n)_{a d}^{\prime}
$$

by assigning $\left\{\left(\mathscr{M}^{\prime}, L\right), \phi_{(n)}(\mathscr{M}, L) ; \operatorname{can}_{n}, \operatorname{Var}_{n}\right\}$ to $(\mathscr{M}, L)$, where $\left(\mathscr{M}^{\prime}, L\right)$ is the restriction of $(\mathscr{M}, L)$ such that $\left(M_{I}^{\prime}, L\right)=\left(M_{I}, L\right)$ for $n \notin I$ (in particular $\psi_{(n)}\left(\mathscr{M}^{\prime}\right.$, $\left.L)=\psi_{(n)}(\mathscr{M}, L)\right)$. Now let $\mathrm{ML}(n)_{a d}^{\prime \prime}$ be the category whose objects are $(\mathscr{M}, L)$ where $\mathscr{M} \in \mathbf{M}(n)$ and $L$ is the filtration of $M_{I}$ for $I \neq \bar{n}$ such that (3.23.7) is satisfied for any $I \neq \bar{n}$ and (3.23.3) is satisfied for any $\mathrm{Gr}_{k}^{L} \mathscr{M}$ for $I \neq \bar{n}$. Then the natural restriction functor induces an equivalence of categories:

$$
\mathrm{ML}(n)_{a d} \stackrel{\sim}{\longrightarrow} \mathrm{ML}(n)_{a d}^{\prime \prime},
$$

i.e. the filtrations $W^{(i)}$ on $M_{\bar{n}}$ are constructed functorially by induction on $i$ using $W^{(i)}$ on the other $M_{I}$ and $W$ on $M_{\bar{n}}$. In fact this is just (3.23.13) if $n=1$, and we can prove it by induction on $n$ using (3.23.13).

3.24. With the notation as in 3.2 and 3.4 , take $(M, F) \in M\left(\mathscr{D}_{X}\right)_{\text {rncqu }}$ (cf. 3.16) with a filtration $W$ such that

(3.24.1) $\quad(M, F, W)$ is the stalk at 0 of the $\mathscr{D}$-Module part of some $\mathscr{M} \in \operatorname{MHW}(X)$,

(3.24.2) the $(n+2)$-filtrations $F, V^{(i)}, W$ on $M$ are compatible.

We define $\left(E_{I}^{v}, F\right),\left(E_{I}^{J}, F\right)$ by $(3.18 .5-6)$ so that $E_{I}^{J}$ has the natural $\mathbb{R}$ structure. Let $L$ be the filtration on $E_{I}^{v}, E_{I}^{J}$ induced by $W$ on $M$. If the condition:

for any order of the coordinates of $X\left(=\Delta^{n}\right)$, the vanishing cycle functors $\psi_{x_{i}}, \phi_{x_{i}, 1}$ are inductively well-defined for $\mathscr{M}$ at the stalk of 0 , is satisfied, we have by (3.18.7)(3.22.4) and (3.24.2):

$E_{I}^{J}$ has the weight filtration $W$ such that $\left(E_{I}^{J} ; F, W, L[|I|-n]\right.$; $\left.N_{1}, \cdots, N_{n}\right) \in \mathrm{MHL}_{a d}^{n}(\mathrm{cf} .3 .22)$ for any order of the coordinates. 
Here $W$ is independent of the order of the coordinates by (3.22.6). From now on, we assume (3.24.1-2) and (3.24.4).

For $g=x^{m}$, we define $\left(\widetilde{E}_{I}^{v} ; F, W\right)$ and $\left(\widetilde{E}_{I}^{J} ; F, W\right)$ by the same way as in 3.18 so that $\widetilde{E}_{I}^{J}$ has the natural $\mathbf{R}$-structure. Let $\widetilde{L}$ be the filtration on $\widetilde{E}_{I}^{v}, \widetilde{E}_{I}^{J}$ induced by $W$ on $M$. Then, by the same argument as in 3.18 , we verify:

$\left(\widetilde{E}_{I}^{J} ; F, W\right)$ is an R-mixed Hodge structure so that $\tilde{L}$ is a filtration of mixed Hodge structures and $\tilde{\operatorname{can}}_{i}, \widetilde{\operatorname{Var}}_{i}, \tilde{N}_{i}$ are morphisms of mixed Hodge structures for $0 \leq i \leq n$.

Assuming (3.24.1-2)(3.24.4), we can prove by induction on $|\bar{m}|$ :

the relative monodromy filtration $L$ of $\left(\widetilde{E}_{I}^{J}, \widetilde{L}\right)$ for $N$ exists so that $\left(\widetilde{E}_{I}^{J} ; F, W, L[|I|-n] ; N_{1}, \cdots, N_{n}\right) \in \mathrm{MHL}_{a d}^{n}$ for any order of the coordinates.

We fix an order of the coordinates. By 3.18-19 and $[6, \S 3]$, the assertion is proved in the pure case, and it is enough to show the existence of $L$ and $W^{(i)}$ in (3.22.4), because we can proceed by induction on the length of $\tilde{L}$ as in the proof of (3.22.2), (3.22.8) and 2.4, where we use the canonical splitting of $\tilde{L}$ on $\mathrm{Gr}_{k}^{L}$ to show that $\left(\mathrm{Gr}_{k}^{L} \widetilde{E}_{I}^{J} ; F, W ; N_{1}, \cdots, N_{n}\right)$ are nilpotent orbits (in particular $W$ is the relative monodromy filtration of $\left(\widetilde{E}_{I}^{J}, L[|I|-n]\right)$ for $\left.\sum N_{i}\right)$. If $|\bar{m}|=1$ and $g$ $=x_{i}^{a}$, the assertion follows from (3.22.6), (3.24.4) and 3.3-4, because $\widetilde{E}_{I}^{v}$ $=E_{I \backslash\{i\}}^{v^{\prime}+v_{0} m}(i \in I, 0 \notin I), E_{I \backslash\{0\}}^{v^{\prime}}(i \in I, 0 \in I)$, and 0 (otherwise) so that $\tilde{N}_{j}=N_{j}(j \neq i)$, $\tilde{N}_{i}=0$ and $N_{i}=a N$ on $\widetilde{E}_{I}^{v}$. Assume $|\bar{m}|>1$. If $I \neq i$, we take $m^{\prime}$ such that $m_{j}^{\prime}$ $=m_{j}(j \neq i)$ and $m_{i}^{\prime}=0$, and define $\widetilde{E}_{I}^{\prime v}$ similarly by replacing $g$ with $g^{\prime}$ $=x^{m^{\prime}}$. Then we have an isomorphism as filtered $\mathbf{C}[N]$-modules:

$$
\left(\widetilde{E}_{I}^{v}, \widetilde{L}\right)=\left(\widetilde{E}_{I}^{\prime v^{\prime}+\left(v_{0} m_{\imath}\right) 1_{\imath}}, \tilde{L}\right)
$$

such that the action of $\tilde{N}_{j}$ is compatible for $j \neq i$ and $\tilde{N}_{i}$ on $\tilde{E}_{I}^{v}$ corresponds to $\tilde{N}_{i}-m_{i} N$ on the right hand side. Therefore the assertion is verified if $I \not \supset \bar{n}$. We can apply (3.23.14) to $\left\{E_{I}^{\phi}\right\}_{I \ni 0},\left\{E_{I}^{\{0\}}\right\}_{I \ni 0},\left\{E_{I}^{\phi}\right\}_{I \ni 0}$, because they belong to $\mathbf{M L}(n)_{a d}^{\prime \prime}$ by the above argument, where (3.23.3) for $\mathrm{Gr}^{L}$ and for $I \neq \bar{n}$ follows from 1.5 and the assertion in the pure case. Thus we get $L, W^{(i)}$ on $\widetilde{E}_{I}^{v}$ also for $I \supset \bar{n}$ such that $L$ is the relative monodromy filtration of $\left(\widetilde{E}_{I}^{v}, \tilde{L}\right)$ for $N$, because the above construction is compatible with $\operatorname{Gr}_{k}^{\tilde{L}}$ and $L$ on $\operatorname{Gr}_{k}^{\tilde{L}} \widetilde{E}_{I}^{v}$ coincides with the monodromy filtration shifted by $k$ by the assertion in the pure case, where $N L_{j} \subset L_{j-2}$ follows from the functoriality of (3.23.14).

As a corollary of (3.24.6) we get:

3.25. Proposition. Let $X$ be a complex manifold of dimension $n, D$ a normal crossing divisor on $X, g$ a holomorphic function on $X$ such that $g^{-1}(0) \subset D$ and $\mathscr{M}$ $=(M, F, K ; W) \in \operatorname{MHW}(X)^{p}$ such that $C h(M) \subset \cup T_{D^{(i)}}^{*} X$, where $D^{(i)}$ are the 
intersections of local irreducible components of D. Assume:

for any point of $D$ there exist local coordinates $\left(x_{1}, \cdots, x_{n}\right)$ such that $D \subset \cup_{i} x_{i}^{-1}(0)$ and (3.24.1-2), (3.24.4) are satisfied for the stalk of $(M$, $F, W)$.

Then the vanishing cycle functors along $g$ are well-defined for $\mathscr{M}$ (cf. 2.2) and $\psi_{g} \mathscr{M}$, $\phi_{g, 1} \mathscr{M}$ satisfy (3.25.1).

Proof. By (3.24.6) the condition (2.3.3) is satisfied. To show (2.2.1) we have to verify the surjectivity of (3.7.7) for $\alpha \leq 0$ by (3.17.2). But this follows from (3.17.3) if $\alpha<0$, and for $\alpha=0$ we apply [19, (3.2.6.4)], (3.16.7)(resp. (3.17.11)) to the $Z$-component of $\mathrm{Gr}_{i}^{W} \mathscr{M}$ such that $Z \subset g^{-1}(0)$ (resp. $Z \leftarrow g^{-1}(0)$ ). Then by 2.4, $\psi_{g} \mathscr{M}, \phi_{g, 1} \mathscr{M}$ belong to $\mathrm{MHW}(X)^{p}$ and (3.24.1) is verified. By (3.22.4) and (3.24.6), (3.24.4) follows from (3.24.1-2). Therefore it remains to show (3.24.2), and we may assume the notations are as in 3.16 . We first show the compatibility of the $n+2$ filtrations $F, V^{(i)}, L$ on $\operatorname{Gr}_{\alpha}^{V} \tilde{M}$ for $-1 \leq \alpha \leq 0$, where $L$ is induced by $W$ on $M$. By $3.20, \operatorname{Gr}_{k}^{L} \operatorname{Gr}_{\alpha}^{V}(\tilde{M}, F) \in \operatorname{MF}\left(\mathscr{D}_{X}\right)_{r n c q u}$ and it is enough to show the surjectivity of

$$
F_{p} V_{v} L_{k} \mathrm{Gr}_{\alpha}^{V} \tilde{M} \longrightarrow F_{p} V_{v} \mathrm{Gr}_{k}^{L} \mathrm{Gr}_{\alpha}^{V} \tilde{M}
$$

by $[19,1.2 .12]$. But this follows from the same argument as the above proof of the surjectivity of (3.7.7), using (3.17.5) for $\alpha<0$ and applying 3.13 to $N: \psi_{g, 1}$ $\rightarrow \psi_{g, 1}(-1)$, because $\operatorname{Coim} N=\phi_{g, 1}$ in the pure case if supp $M$ $\phi g^{-1}(0)$. Therefore $L$ is a filtration of $\operatorname{Gr}_{\alpha}^{V}(\tilde{M}, F)$ in the exact category $\operatorname{MF}\left(\mathscr{D}_{X}\right)_{\text {rncqu }}$, where a strict morphism in this category is strict for the $n+1$ filtrations $F, V^{(i)}$ by definition. Then (3.18.8) is a filtered isomorphism also in the mixed case, because the inclusion $\subset$ is clear for each $F_{p}$ and the equality holds on $\mathrm{Gr}_{k}^{L}$ by the above argument. Now we can show the compatibility of the $n+3$ filtrations $F, V^{(i)}, W, L$ on $\mathrm{Gr}_{\alpha}^{V} \tilde{M}$, which completes the proof of 3.25. The pure case was proved in 3.20 , because the assertion for $\operatorname{Gr}_{0}^{V}$ follows from the strictness of

$$
N:\left(\mathrm{Gr}_{-1}^{V} \tilde{M} ; F, V^{(i)}, W\right) \longrightarrow\left(\mathrm{Gr}_{-1}^{V} \tilde{M} ; F[-1], V^{(i)}, W[2]\right),
$$

where the case $\operatorname{supp} M \subset g^{-1}(0)$ is clear by $[19,3.2 .6]$. Then by the same argument as the proof of (2.4.1), it is enough to show the strictness of

$$
\begin{aligned}
\left(N^{i+1}, \mathrm{id}\right):\left(L_{k-1} M^{\prime}, F[i+1], V^{(j)}\right) & \oplus\left(W_{-i-2+k} L_{k-1} M^{\prime}, F, V^{(j)}\right) \\
& \longrightarrow\left(L_{k-1} M^{\prime}, F, V^{(j)}\right)
\end{aligned}
$$

as $(n+1)$-filtered morphism, where $M^{\prime}=\operatorname{Gr}_{\alpha}^{V} \tilde{M}$. But this can be reduced to the strictness of $\mathrm{Gr}_{v}^{V}$ of the morphism, and follows from (3.24.5) and (3.18.8) in the mixed case proved just above. 
3.26. Proposition. Let $X, D$ and $\mathscr{M}$ be as in 3.25, and $D^{\prime}$ a union of irreducible components of D. Put $j^{\prime}: U^{\prime}:=X \backslash D^{\prime} \rightarrow X$. Assume (3.25.1). Then $j_{!}^{\prime} j^{\prime-1} \mathscr{M}$, $j_{*}^{\prime} j^{\prime-1} \mathscr{M} \in \mathrm{MHW}(X)^{p}$ are well-defined (cf. 2.17) and they satisfy also (3.25.1).

Proof. By 2.11 and 3.25, the assertion is local and we may assume $D^{\prime}$ is locally irreducible by factorizing $j^{\prime}$ locally because $j_{!}^{\prime}, j_{*}^{\prime}$ are unique once an ideal of $D^{\prime}$ is fixed (cf. 2.11) and they satisfy the conditions for $j_{!}^{\prime}, j_{*}^{\prime}$ for any ideal if the condition (3.25.1) is verified (cf.3.25). Therefore we may assume $X=\Delta^{n}, D^{\prime}$ $=\left\{x_{n}=0\right\}$ and $D \cap\left(\Delta^{*}\right)^{n}=\phi$. We define $\tilde{\mathscr{M}}=j_{!}^{\prime} j^{\prime-1} \mathscr{M}$ in $\operatorname{MHW}(X)^{p}$ by 2.11 using the product $X=D^{\prime} \times \Delta$. Then (3.24.4) follows from [13,5.5.4] and it remains to show (3.24.2). We have by definition:

$$
\text { the } n+2 \text { filtrations } F, V^{(i)}, W \text { are compatible on } V_{<0}^{(n)} \tilde{M}=V_{<0}^{(n)} M \text {. }
$$

We first show

the $n+1$ filtrations $F, V^{(i)}(i \neq n), W$ are compatible on $\operatorname{Gr}_{\alpha}^{V^{(n)}} \tilde{M}$ for any $\alpha \geq 0$.

Clearly it is enough to show the assertion for $\alpha=0$. Put $\left(M^{\prime}, L\right)=\operatorname{Gr}_{-1}^{V^{(n)}}(\tilde{M}$, $W[-1])$ and $\left(M^{\prime \prime}, L\right)=\operatorname{Gr}_{0}^{V^{(n)}}(\tilde{M}, W)$, and let $L^{\prime}$ be the filtration on $M^{\prime}$ such that

$$
\partial_{t}:\left(M^{\prime} ; F, V^{(i)}(i \neq n), L^{\prime}\right) \longrightarrow\left(M^{\prime \prime} ; F, V^{(i)}(i \neq n), L\right)
$$

becomes an $(n+1)$-filtered isomorphism. Then we have

$$
L_{k}^{\prime} M^{\prime}=\operatorname{Ker}\left(N_{n}: L_{k-1} M^{\prime}+W_{k} M^{\prime} \longrightarrow M^{\prime} / L_{k-1} M^{\prime}\right)
$$

by (2.8.4), where $W$ is the relative monodromy filtration of $\left(M^{\prime}, L\right)$ for $N_{n}$. By the proof of $3.25, W, L$ are 2 filtrations of $\left(M^{\prime}, F\right)$ in $\operatorname{MF}\left(\mathscr{D}_{\Delta^{n-1}}\right)_{r n c q u}$. In particular the $n+2$ filtrations $F, V^{(i)}, L, W$ are compatible. To show (3.26.2) it is enough to show the strictness of the morphism in (3.26.3) as a morphism in $\operatorname{MF}\left(\mathscr{D}_{\Delta^{n-1}}\right)_{r n c q u}$. By 3.13, it is reduced to that of $\mathrm{Gr}_{v}^{V}$, and follows from the fact that $W, L$ underlie filtrations in $\operatorname{MHW}\left(\Delta^{n-1}\right)$. Therefore, to show (3.24.2), it is enough to check the surjectivity of

$$
F_{p} V_{v^{\prime}}^{\prime} W_{k} V_{\alpha}^{(n)} \tilde{M} \longrightarrow F_{p} V_{v^{\prime}}^{\prime} W_{k} \mathrm{Gr}_{\alpha}^{V^{(n)}} \tilde{M} \quad \text { for } \alpha \geq 0
$$

by $(3.26 .1)$ and $[19,1.2 .9]$, where $v^{\prime} \in \mathbb{Q}^{n-1}$ and $V_{v^{\prime}}^{\prime}=\cap V_{v_{t}}^{(i)}$. But this follows from the same argument as the proof of 2.9 if $\alpha=0$, and the other case is clear by the action of $\partial_{t}$, where $t=x_{n}$. We have a similar argument for $j_{*}^{\prime} j^{\prime-1} \mathscr{M}$. This completes the proof of 3.26 .

Remark. With the notation as above, let $g$ be a meromorphic function on $X$ such that $g$ is holomorphic on $U^{\prime}$ and $g^{-1}(0) \subset D$. Then $j_{!}^{\prime}, j_{*}^{\prime}$ are well-defined for $\psi_{g} j^{\prime-1} \mathscr{M}, \phi_{g, 1} j^{\prime-1} \mathscr{M}$ if $\mathscr{M}$ satisfies (3.25.1), and (3.25.1) is stable by the 
compositions of $j_{!}^{\prime}, j_{*}^{\prime}$ and $\psi_{g} j^{\prime-1}, \phi_{g, 1} j^{\prime-1}$. In fact, we can reduce to the case $g$ holomorphic on $X$, because we can multiply $g$ by $x_{i}^{a}$ locally by (3.7.8) if $T^{a}$ becomes unipotent where $T$ is the monodromy of $\psi_{g}$.

3.27. Theorem. Let $X$ be a complex manifold of dimension $n$, and $U a$ Zariski open dense subset. Then we have an equivalence of categories

$$
\operatorname{VMHS}(U)_{X}^{a d} \stackrel{\longrightarrow}{\longrightarrow} \operatorname{MHM}(U)_{X}^{p s}
$$

where the right hand side is the category of the polarizable smooth mixed Hodge Modules on $U$ extendable to $\operatorname{MHM}(X)^{p}$, and the left hand side is the category of admissible variations of mixed Hodge structures on $U$ relative to $X$ (cf. [13]). Here smooth means that the underlying perverse sheaf is a local system up to $a$ shift, and the functor is given by $((L, F), K ; W) \mapsto\left((L, F) \otimes\left(\Omega_{U}^{n}, F\right)\right.$, $K[n] ; W[n])$.

Proof. We may assume $D=X \backslash U$ is a normal crossing divisor. Let $j: U \rightarrow X$ be the natural inclusion, and take $\left((L, F), K^{\prime}[-n] ; W[-n]\right) \in \operatorname{VMHS}(U)_{X}^{a d}$. Put $\left(M^{\prime}, F\right)=\left(\Omega_{u}^{n}, F\right) \otimes(L, F)$ so that $\mathscr{M}^{\prime}=\left(\left(M^{\prime}, F\right), K^{\prime} ; W\right) \in \operatorname{MHW}(U)^{p}$ (cf. [19, 5.4.3]). We first show the existence of $\mathscr{M}=((M, F), K, ; W) \in \operatorname{MHW}(X)^{p}$, denoted by $j_{*} \mathscr{M}^{\prime}$, such that $K=j_{*} K^{\prime}$ and $(M ; F, W)$ satisfies (3.25.1). By 2.11, the assertion is local, because $j_{*} \mathscr{M}^{\prime}$ is unique by 3.26. Therefore we may assume $X=\Delta^{n}$ as in the proof of 3.26. We define $(M, F)=j_{*}^{\text {reg }}\left(M^{\prime}, F\right)$ as in 3.10. Assume $D=U_{i \leq r} D_{i}$ and we factorize $j$ by $j=j_{1} \cdots j_{r}$, where $j_{i}: U_{i} \rightarrow U_{i-1}$ and $X \backslash U_{i}=\cup_{k \leq i} D_{k}$. We define $j_{*}$ by the composition $\left(j_{1}\right)_{*} \cdots\left(j_{r}\right)_{*}$ using 2.11 , if (2.7.2-4) are verified inductively. Here $W$ is determined inductively by (2.8.4), (2.11.2) and (3.1.3), (3.2.2). Therefore we may forget the $\mathbb{Q}$-structure. Because (2.7.4) is verified inductively by $[13,5.5 .4]$, we get $W$ on $M \in M\left(\mathscr{D}_{X}\right)_{\text {rncqu }}$. To show (2.7.2-3), it is enough to verify the compatibility of the $n+2$ filtrations $F$, $V^{(i)}, W$ at every point of $D$. Therefore we may assume the notations are as in 3.16. By [13] they are compatible on $V_{<0} M$ by taking a finite covering. We define the filtrations $W(i)$ on $M$ by

$$
\mathrm{DR}\left(W(i)_{k} M\right)=\left(j_{i}^{\prime}\right)_{*} j_{i}^{\prime-1} \mathrm{DR}\left(W_{k} M\right)
$$

where $j_{i}^{\prime}: U_{i} \rightarrow X$. Then $W=W(0)$, and for $\left\{\left(E_{I}^{v}, W(i)\right)\right\}=\Psi_{\mathscr{D}}^{n}(M, W(i))$, we have $W(i)_{k} E_{I}^{v}=W_{k} E_{I}^{v}$ for $I \cap\{1, \cdots, i\}=\phi$ and $\operatorname{Var}_{j}$ are bijective for $j \leq i$. By the isomorphism

$$
\begin{aligned}
x_{1} \cdots x_{r}:\left(V_{0} M ; F\right. & \left.V^{(i)}, W(r)\right) \\
& \stackrel{\sim}{\longrightarrow}\left(V_{-1} M ; F, V^{(i)}[1](i \leq r), V^{(i)}(i>r), W\right)
\end{aligned}
$$

we get the compatibility of $F, V^{(i)}, W(r)$ on $V_{0} M$ and then on $M$ using the same 
argument as in the proof of 2.9 and (3.16.14), because $\mathrm{Gr}_{k}^{W(r)}(M, F)$ $=j_{*}^{\text {reg }} \mathrm{Gr}_{k}^{W}\left(M^{\prime}, F\right)$. We can verify inductively the compatibility of $F, V^{(i)}, W(j)$ by the same argument as the proof of (3.26.2). In fact $W(j)=W(j-1)$ on $V_{<0}^{(j)} M$ and the $n+2$ filtrations $F, V^{(i)}(i \neq j), \tilde{W}, W(j)$ are compatible on $\mathrm{Gr}_{-1}^{V^{(j)}} M$ by induction on the length of $W(j)$ where $\widetilde{W}$ is the relative monodromy filtration of $\mathrm{Gr}_{-1}^{V^{(j)}}(M, W(j)[-1])$ for $N_{j}$. Then we get the compatibility of $F$, $V^{(i)}(i \neq j), W(j-1)$ on $\mathrm{Gr}_{0}^{V^{(j)}} M$ and that of $F, V^{(i)}, W(j-1)$ on $V_{0}^{(j)} M$ and then on $M$.

Now we show $j_{*} \mathscr{M} \in \operatorname{MHM}(X)$. Because the construction of $j_{*}$ is compatible with the smooth pull-backs, it is enough to verify the iterations of $j_{!}^{\prime} j^{\prime-1}, j_{*}^{\prime} j^{\prime-1}, \psi_{g}, \phi_{g, 1}$. But we can reduce to the normal crossing case as in the proof of 3.8 by Hironaka's desingularization and 2.5, 2.12, 2.14. Then the assertion follows from 3.25-26, and this completes the proof of 3.27.

Remark. In the algebraic case, we can show the equivalence of categories:

$$
\operatorname{VMHW}(X)^{a d} \stackrel{\sim}{\longrightarrow} \operatorname{MHM}(X)^{s}
$$

for a smooth separated algebraic variety $X$, where $\operatorname{MHM}(X)$ will be defined in $4.2, s$ is for smooth as in 3.27 , and the left hand side is the category of algebraic variations of mixed Hodge structure (in particular the underlying connection has regular singularities at infinity) satisfying the admissibility condition defined by the curve test (cf.[13][22]). In fact this follows from the same argument as the proof of 3.27, combined with the remark after 3.26. Then this equivalence and $2.8,2.28$ imply by induction on $\operatorname{dim} X$ :

$$
\operatorname{MHM}(X) \stackrel{\sim}{\longrightarrow} \operatorname{MHM}\left(X^{a n}\right)_{X^{a n}}^{p}
$$

for any separated algebraic variety $X$ and any completion $\bar{X}$ of $X$, where an means the associated analytic space.

By a similar argument and [13], we have

3.28. Theorem. The polarizable mixed Hodge Modules are stable by the external products 囚.

Proof. By 3.21, MHW $(X)$ are stable by $区$. We can verify that $\mathscr{M}$ $凶 \mathscr{N} \in \operatorname{MHM}(X \times Y)$ for $\mathscr{M} \in \operatorname{MHM}(X)^{s}$ and $\mathscr{N} \in \operatorname{MHM}(Y)$ by induction on $\operatorname{dim} \operatorname{supp} \mathscr{N}$ using the equivalences of categories 2.8 and 2.28 , because the assertion follows from [13] in the case of admissible variation of mixed Hodge structure. Then the general case follows from the same argument as abeve.

3.29. Remark. Let $X$ and $D$ be as in $2.5, \operatorname{MHW}(X ; D)^{p}$ the full subcategory of $\operatorname{MHW}(X)^{p}$ whose objects satisfy the assumptions in 3.25 , and $\operatorname{MHM}(X ; D)^{p}$ the full subcategory of $\operatorname{MHM}(X)^{p}$ such that the characteristic varieties of its objects 
satisfy the assumption in 3.25 . Then we have

$$
\operatorname{MHW}(X ; D)^{p}=\operatorname{MHM}(X ; D)^{p} \quad\left(\text { in } \operatorname{MHW}(X)^{p}\right) .
$$

In fact, the assertion is local, and we can prove it by induction on the dimension of the support using $2.8,2.28$ and the functors $\left(j_{i}\right)_{*} j_{i}^{-1}$ as in the proof of 3.27.

\section{§4. Algebraic Case}

In this section, algebraic varieties are defined over $\mathbb{C}$ and separated reduced.

4.1. Let $X$ be an algebraic variety. For a subfield $A$ of $\mathbb{C}$, let $\operatorname{Perv}\left(A_{X}\right)$ be the full subcategory of $\operatorname{Perv}\left(A_{X^{a n}}\right)$ whose objects have algebraic stratifications such that the restrictions of their cohomology sheaves to the strata are local systems. If $X$ is smooth, $\mathrm{MF}_{h}\left(\mathscr{D}_{X}\right)$ denotes the category of filtered (algebraic) $\mathscr{D}_{X}$-Modules $(M, F)$ such that $M$ is regular holonomic [4] [5] and $\operatorname{Gr}^{F} M$ is coherent over $\operatorname{Gr}^{F} \mathscr{D}_{X}$. Then we have the functor $\mathrm{DR}: \mathrm{MF}_{h}\left(\mathscr{D}_{X}\right) \rightarrow \operatorname{Perv}\left(\mathbb{C}_{X}\right)$ as usual, and we repeat the definition of $\mathrm{MF}_{h}\left(\mathscr{D}_{X}, \mathbb{Q}\right), \mathrm{MF}_{h} W\left(\mathscr{D}_{X}, \mathbb{Q}\right), \mathrm{MH}_{(Z)}(X, n)^{(p)}$ and $\mathrm{MHW}(X)^{(p)}$, etc. in $[19, \S 5.1-2]$, where $Z$ is an irreducible closed subvariety and $U, g$ in the definition of Hodge Module and polarization are algebraic. By the same argument, the main results in $[19, \S 5]$ remain valid, e.g.

$$
\begin{aligned}
& \operatorname{MH}_{(Z)}(X, n)^{(p)}, \operatorname{MHW}(X)^{(p)} \text { are abelian categories, } \\
& \mathbb{Q}_{X}^{H}\left[d_{X}\right] \in \operatorname{MH}\left(X, d_{X}\right)^{p}\left(\text { cf. } 2.17 \text { for the definition of } \mathbb{Q}_{X}^{H}\left[d_{X}\right]\right. \text { ) } \\
& \text { for } f: X \rightarrow Y \text { a projective morphism and for } \mathscr{M} \in \mathbb{M H}(X, n)^{p} \text { (or } \\
& \left.\operatorname{MHW}(X)^{p}\right), f_{*}(M, F) \text { is strict and } \mathscr{H}^{j} f_{*} \mathscr{M} \in \mathbb{M H}(X, n+j)^{p} \text { (or } \\
& \text { MHW } \left.(X)^{p}\right), \text { cf. } 2.14 \text { for the mixed case, }
\end{aligned}
$$

where $(M, F)$ is the underlying filtered $\mathscr{D}_{X}$-Module and we use an affine open covering and the associated Cech complex for the definition of $f_{*}(M, F)$. (If one prefers, the can use filtered $\mathscr{D}_{X^{a n}}$-Modules $(M, F)$ such that $\operatorname{DR}(M, F) \in \operatorname{Perv}\left(\mathbb{Q}_{X}\right)$ in the definition of $\mathrm{MH}(X, n)^{(p)}$, etc., because the final result is the same by GAGA and the extendability.) Moreover the results in $\$ 2$ (except for the definition of $\operatorname{MHM}(X)^{(p)}$ ) are valid in the algebraic case. (Note that the mixed Hodge Modules are assumed polarizable and extendable in the algebraic case, cf. 4.2.) For example, if $X$ is singular, we define $\operatorname{MH}(X, n)^{(p)}, \mathrm{MHW}(X)^{(p)}$ as in 2.1 using local embeddings. Then (4.1.1) and (4.1.3) remain true for singular $X$, $Y$. For a function $g$ on $X,\left(i_{g}\right)_{*} \mathscr{M} \in \operatorname{MHW}(X \times \mathbb{C})^{(p)}$ is well-defined for $\mathscr{M} \in \operatorname{MHW}(X)$ and we can define the condition:

(4.1.4) the vanishing cycle functors along $g$ are well-defined for $\mathscr{M}$,

as in 2.3 (i.e. assume (2.2.8) (2.3.3) which are independent of the local embedding of $X$ and the local extension of $g$.) Then 2.4 is also valid and we can define 
$\psi_{g} \mathscr{M}$ and $\phi_{g, 1} \mathscr{M}$, if (4.1.4) is satisfied. By 2.5-6 and 2.14, we have

(4.1.5) the condition (4.1.4) is stable by the dual functor $\mathbb{D}$, subquotients in $\operatorname{MHW}(X)^{(p)}$ and projective direct images as in (4.1.3) (in the polarizable case), and (2.6.2) is valid if (4.1.4) is satisfied.)

Let $D$ be a closed subset of $X$. We say $D$ is a weakly locally principal divisor (abbreviated by w.l.p.d) if

(4.1.6) locally there is a function $g$ (called a local equation of $D$ ) such that $D=g^{-1}(0)_{\text {red }}$,

Here $D$ might contain an irreducible component of $X$, i.e. $g$ might be zero on this component. Let $j: U \rightarrow X$ be an open immersion such that $X \backslash U$ is a w.l.p.d. (in particular, $j$ is affine). We say $j_{!} \mathscr{M}^{\prime}$ (resp. $j_{*} \mathscr{M}^{\prime}$ ) exists for $\mathscr{M}^{\prime} \in \operatorname{MHW}(U)^{(p)}$, if

(4.1.7) there exists an extension $j_{!} \mathscr{M}^{\prime}$ (resp. $\left.j_{*} \mathscr{M}^{\prime}\right) \in \mathrm{MHW}(X)^{(p)}$ of $\mathscr{M}^{\prime}$ such that the vanishing cycle functors along any local equation of $D$ are well-defined and its underlying perverse sheaf is of the form $j_{!} K^{\prime}$ (resp. $\left.j_{*} K^{\prime}\right)$.

(Here $K^{\prime}$ is necessarily the underlying perverse sheaf of $\mathscr{M}^{\prime}$.) By the same argument as in 2.8 and 2.11 , we have

(4.1.8) $\quad j_{*} \mathscr{M}^{\prime}$ (resp. $j_{*} \mathscr{M}^{\prime}$ ) is unique and functorial (for $M^{\prime}$ satisfying (4.1.7)) and we have the natural (unique) morphism inducing the identity on $U$ :

$$
j_{!} j^{-1} \mathscr{M} \longrightarrow \mathscr{M}\left(\operatorname{resp} . \mathscr{M} \longrightarrow j_{*} j^{-1} \mathscr{M}\right)
$$

for $\mathscr{M} \in \mathrm{MHW}(X)^{(p)}$ such that the vanishing cycle functors along any local equation of $D$ are well-defined for $\mathscr{M}$ and (4.1.7) is satisfied for $j^{-1} \mathscr{M}$.

Here we apply 2.8 and 2.11 to $\left(i_{g}\right)_{*} M^{\left({ }^{\prime}\right)}$. Then $j_{!} \mathscr{M}^{\prime}$ and $j_{*} \mathscr{M}^{\prime}$ are uniquely determined, once a local defining Ideal of $D$ (generated by $g$ ) is fixed, but they might depend on the Ideal. We eliminate this ambiguity by assuming the condition for any $g$. By 2.12 we have

(4.1.9) the condition (4.1.7) is stable by subquotients in $\operatorname{MHW}(X)^{p}$,

where the polarizability is used to show the splitting of $\mathrm{Gr}^{W}$ of (2.12.1).

4.2. Let $X$ be an algebraic variety. For the definition of the category of Mixed Hodge Modules $\mathrm{MHM}(X)$, we consider all the smooth varieties $Y$, open sets $U_{i}$ of $U_{i-1}^{\prime}$, open immersions $j_{i}: U_{i} \rightarrow U_{i}^{\prime}$ such that $U_{i}^{\prime} \backslash U_{i}$ are w.l.p.d. (cf. (4.1.6)), and functions $g_{i}$ on $U_{i}$ for $i>0$, where $U_{0}^{\prime}=X \times Y$. Then $\operatorname{MHM}(X)$ is the full subcategory of $\mathrm{MHW}(X)^{p}$ defined by the following condition on $\mathscr{M} \in \mathrm{MHW}(X)^{p}$ for any $Y, U_{i}, U_{i}^{\prime}, g_{i}(i>0)$ as above (inductively on $i$ ): 
(4.2.1) the vanishing cycle functors along $g_{i}$ are well-defined for $\left.\mathscr{M}_{i-1}\right|_{U_{i}}$ (cf. (4.1.4)) and $\left(j_{i}\right)_{!},\left(j_{i}\right)_{*}$ exist (cf. (4.1.7)) for both $\left.\psi_{g_{1}} \mathscr{M}_{i-1}\right|_{U_{i}^{\prime}}$ and $\left.\phi_{g_{1}, 1} \mathscr{M}_{i-1}\right|_{U_{i}}$ where $\mathscr{M}_{0}=\mathscr{M} \otimes \mathbb{Q}_{Y}^{H}\left[d_{Y}\right]$ and $\mathscr{M}_{i}$ is obtained by operating $\left(j_{i}\right)_{!}$or $\left(j_{i}\right)_{*}$ after $\psi_{g_{1}}$ or $\phi_{g_{1}, 1}$ to $\left.\mathscr{M}_{i-1}\right|_{U_{l}}(i>0)$. (Here $\mathscr{M}_{0} \in \mathrm{MHW}(X \times Y)^{p}$ is also assumed.)

By definition we have

(4.2.2) $\operatorname{MHM}(X)$ is stable by the operations in (4.2.1): $₫ \mathbb{Q}_{Y}^{H}\left[d_{Y}\right], j_{!}, j_{*}, \psi_{g}$, $\phi_{g, 1}$, where $Y$ is smooth and $j$ is as in (4.1.7).

because $\otimes \mathbb{Q}_{Y}^{H}\left[d_{Y}\right]$ commutes with these functors and $\phi_{g, 1}=$ id if $g=0$. By (4.1.1), (4.1.5) and (4.1.8-9) we have

(4.2.3) $\operatorname{MHM}(X)$ is an abelian full subcategory of $\operatorname{MHW}(X)^{p}$, stable by the dual $\mathbb{D}$ and subquotients in $\operatorname{MHW}(X)$, and $\mathbb{D} j_{!}=j_{*} \mathbb{D}$ for $j$ as in (4.1.7).

Let $i: X \rightarrow Y$ be a closed immersion of algebraic varieties. By the remark after (4.1.4), we have a functor:

$$
i_{*}: \operatorname{MHM}(X) \rightarrow \operatorname{MHM}_{X}(Y)
$$

where $\operatorname{MHM}_{X}(Y)$ is the full subcategory of $\operatorname{MHM}(Y)$ whose objects have support in $X$. To show that (4.2.4) is an equivalence of categories, we give the second definition of mixed Hodge Module as follows:

Let $X$ be a projective variety. Then $\operatorname{MHM}(X)^{\prime}$ is the full subcategory of MHW $(X)^{p}$ such that the condition (4.2.1) is satisfied for any smooth projective $Y$ and $U_{i}, U_{i}^{\prime}, g_{i}$ such that $U_{0}^{\prime}=X \times Y$. For an open set $U$ of $X, \operatorname{MHM}(U)_{X}^{\prime}$ is the full subcategory of $\mathrm{MHW}(U)^{p}$ whose objects are the restrictions of those of $\operatorname{MHM}(X)^{\prime}$. We shall see that $\operatorname{MHM}(U)_{X}^{\prime}$ depends only on $U$ and define $\operatorname{MHM}(U)^{\prime}=\operatorname{MHM}(U)_{X}^{\prime}$. Then for any algebraic variety $X, \operatorname{MHM}(X)^{\prime}$ is defined using an affine open covering and we shall verify the independence of the covering and $\operatorname{MHM}(X)=\operatorname{MHM}(X)^{\prime}$. We first note

(4.2.5) for a projective variety $X, \operatorname{MHM}(X)^{\prime}$ satisfies (4.2.2-3) by replacing $j_{!}, j_{*}$ with $j_{!} j^{-1}, j_{*} j^{-1}$ and $\psi_{g}, \phi_{g, 1}$ with the compositions of $j_{!}, j_{*}$ with $\psi_{g} j^{-1}, \phi_{g, 1} j^{-1}$, where the complement of $\operatorname{Im} j$ is a w.l.p.d. on $X$ and $Y$ is smooth projective.

Then this implies

(4.2.6) for a projective variety $X$ with an open covering $X=\cup U_{i}$, $\mathscr{M} \in \mathrm{MHW}(X)$ belongs to $\operatorname{MHM}(X)^{\prime}$ iff its restriction to $U_{i}$ belongs to $\operatorname{MHM}\left(U_{i}\right)_{X}^{\prime}$ for any $i$.

In fact we may assume $X \backslash U_{i}$ is a w.l.p.d. and $U_{i}$ are finite (taking a refinement of 
the covering), where we assume $\left.M\right|_{U_{1}} \in \operatorname{MHM}\left(U_{i}\right)_{X}^{\prime}$ because the converse is trivial. Then the Ĉech complex associated to this covering (using $j_{\text {! }}$ or $j_{*}$ ) gives a resolution of $\mathscr{M}$ (in $\mathrm{MHW}(X)$ ) by objects of $\operatorname{MHM}(X)^{\prime}$, whose differential is induced by (4.1.8). Therefore $\mathscr{M} \in \operatorname{MHM}(X)^{\prime}$ by its stability by Ker, Coker in $\operatorname{MHW}(X)$.

To show the equivalence of categories (4.2.4) for the second definition, i.e. for $\operatorname{MHM}(X)^{\prime}$, etc. (where $X, Y$ are projective), it is enough to show the stability of $\operatorname{MHM}_{X}(Y)^{\prime}$ by the operations in the definition of $\operatorname{MHM}(X)^{\prime}$. Because $\operatorname{MHW}(X)=\operatorname{MHW}_{X}(Y)$ and the condition (4.1.4) and the definition of $\psi_{g}, \phi_{g, 1}$ are independent of the closed immersion (cf. the remark after (4.1.4)), it is enough to check the stability by the composition of $j_{!}, j_{*}$ with $\psi_{g} j^{-1}, \phi_{g, 1} j^{-1}$, where $j: U \rightarrow X$ is an open immersion such that $X \backslash U$ is a w.l.p.d. and $g$ is a function on $U$. Let $Y=\cup V_{i}$ be an open covering with functions $h_{i}$ on $V_{i}$ and $g_{i}$ on $V_{i} \backslash h_{i}^{-1}(0)$ such that $U \cap V_{i}=X \cap\left(V_{i} \backslash h_{i}^{-1}(0)\right), g_{i}\left|U \cap V_{i}=g\right| U \cap V_{i}$ and $Y \backslash V_{i}$ is a w.l.p.d. Then $Y \backslash\left(V_{i} \backslash h_{i}^{-1}(0)\right)$ is also a w.l.p.d. and the assertion is verified locally by (4.2.5) applied to $V_{i} \backslash h_{i}^{-1}(0) \rightarrow Y$ and $g_{i}$. Therefore (4.2.6) implies the assertion, i.e. we get the equivalence of categories:

$$
i_{*}: \operatorname{MHM}(X)^{\prime} \stackrel{\sim}{\longrightarrow} \operatorname{MHM}_{X}(Y)^{\prime}
$$

for $i: X \rightarrow Y$ a closed immersion of projective varieties. Then this implies the stability by pull-backs by the same argument as in 2.19 :

for a morphism of projective varieties $f: X \rightarrow Y, \mathscr{H}^{j} f_{!}=\mathscr{H}^{j} f_{*}, \mathscr{H}^{j} f^{!}$, $\mathscr{H}^{j} f^{*}$ are well-defined as functors between $\operatorname{MHM}(X)^{\prime}$ and $\operatorname{MHM}(Y)^{\prime}$, and they are compatible with the functors on the underlying perverse sheaves.

In fact, the assertion for direct images follows from (4.1.5). For pull-backs, we use a factorization $f=p \circ i$ such that $p=\operatorname{pr}_{1}: Y \times Z \rightarrow Y$ with $Z$ smooth and $i$ is a closed immersion. Then for a closed immersion we take an open covering $Y \backslash X=\cup U_{i}$ such that $Y \backslash U_{i}$ is a w.l.p.d. (by the same argument as in the proof of (4.2.7)). For the independence of factorization, we have to show (2.19.1) in the case $f$ is a closed immersion (and $p$ as above). We extend local equations of $\operatorname{Im} i$ in $\operatorname{Im} f \times Z$ to $Y \times Z$ and get locally an étale morphism $Y \times Z \rightarrow Y \times \mathbb{C}^{d_{Z}}$ over $Y$. Therefore the assertion is reduced to the case $Z=\mathbb{C}^{n}$ and $\operatorname{Im} i=\operatorname{Im} f$ $\times\{0\}$, and it follows from the same argument as in 2.19 .

By (4.2.8), $\operatorname{MHM}(U)_{X}^{\prime}$ is independent of $X$ and $\operatorname{MHM}(U)^{\prime}$ is welldefined. Moreover it is an abelian full subcategory of $\mathrm{MHW}(U)$, because we can take $X$ such that $X \backslash U$ is a locally principal divisor and use $j_{!}$or $j_{*}$ for the extension. Similarly it is stable by $\psi_{g}, \phi_{g, 1}$ for a function $g$ on $U, \otimes \mathbf{Q}_{Z}^{H}\left[d_{Z}\right]$ for a smooth quasi-projective $Z$, and subquotients in $\mathrm{MHW}(U)$, and (4.2.8) holds for a morphism of quasi-projective varieties, where $\mathscr{H}^{j} f_{!} \neq \mathscr{H}^{j} f_{*}$ (if $f$ is not proper) 
and they are defined as in 2.18 (the independence of factorization follows from the uniqueness of $j_{\text {! }}$ and $j_{*}$ ).

For the well-definedness of $\operatorname{MHM}(X)^{\prime}$ for a general $X$ and for the compatibility with its definition for a quasi-projective variety, it is enough to check (4.2.6) for $X$ quasi-projective. We take a projective completion $\bar{X}$ such that $\bar{X} \backslash X$ is a locally principal divisor, and take a refinement of the covering $X$ $=\cup U_{i}$ such that $\bar{X} \backslash U_{i}$ is a w.l.p.d. Then the Cech complex gives a resolution of $j_{!} \mathscr{M}$ or $j_{*} \mathscr{M}$ where $j: X \rightarrow \bar{X}$, and we get the assertion as in the proof of (4.2.6). We now show

$$
\operatorname{MHM}(X)=\operatorname{MHM}(X)^{\prime} .
$$

The inclusion $\subset$ is clear by definition. For the converse we have to show the stability of $\operatorname{MHM}(X)^{\prime}$ by the operations used in (4.2.1). By definition the stability by $\otimes \mathbb{Q}_{Y}^{H}\left[d_{Y}\right]$ and vanishing cycle functors follows from the above argument in the quasi-projective case. For the stability by $j_{!}, j_{*}$ for an open immersion $j: U \rightarrow X$ as in (4.2.1) we may assume $X$ is affine and $X \backslash U=g^{-1}(0)_{\text {red }}$ by the uniqueness of $j_{!}, j_{*}$ (taking a covering of $X$ ). Then the assertion is clear.

As a corollary of (4.2.9) we get the equivalence of categories (4.2.4), because it can be reduced to the case $Y$ affine (and then projective). We can also define $\mathscr{H}^{j} f_{!}, \mathscr{H}^{j} f_{*}, \mathscr{H}^{j} f^{!}, \mathscr{H}^{j} f^{*}$ for any morphism of algebraic varieties. (For the direct images it is enough to define $\mathscr{H}^{j} f_{*} \mathscr{M}$ in the case $f$ proper and $\mathscr{M}$ pure, cf. 2.16. But this can be reduced to the projective case by Chow's lemma, combined with the stability by pull-backs and subquotients and Deligne's uniqueness of the decomposition.) But we give a better definition later, cf. 4.3.

By (4.2.2) and (4.2.4) we can define $\xi_{g}$ as in 2.22 and get the results in (2.e) similarly. Moreover, for a closed immersion of algebraic varieties $i: X \rightarrow Y$ we have an equivalence of categories

$$
i_{*}: D^{b} \mathrm{MHM}(X) \stackrel{\sim}{\rightarrow} D_{X}^{b} \operatorname{MHM}(Y)
$$

In fact it is enough to check the effaceability as in $[3,(3.1 .16)] . \quad$ By the next assertion, we can reduce to the case $Y$ affine using an affine covering as in [1]. Then we may assume $X=g^{-1}(0)_{\text {red }}$ by induction. We have also

(4.2.11) Let $j: U \rightarrow X$ be an affine open immersion. Then we have the exact functors

$$
j_{!}, j_{*}: \operatorname{MHM}(U) \rightarrow \operatorname{MHM}(X)
$$

compatible with the functors on the underlying perverse sheaves and representing the adjoint functors of $j^{-1}$, i.e. we have the natural functorial morphisms $j_{!} j^{-1} \longrightarrow$ id and id $\longrightarrow j_{*} j^{-1}$.

By uniqueness we may assume $X$ affine. Then the assertion is proved if $U$ 
$=X \backslash g^{-1}(0)_{\text {red }}$. We prove the assertion using the associated Čech complex to a covering of $U$ by such open subsets (as in the proof of (4.2.6) for $X$ quasiprojective). Here the condition $j$ affine is used only for the acyclicity of $j_{\text {! }}$, $j_{*}$ (i.e. they preserve the perversity).

By the same argument as in 3.8-9, we have

$$
\operatorname{MHM}(p t)=\{\text { polarizable Q-mixed Hodge structures }\}
$$

Let $\operatorname{MHM}(X)_{e p}$ be the full subcategory of $\operatorname{MHM}(X)$ whose objects $\mathscr{M}$ satisfy the condition:

$$
\text { the mixed Hodge Modules are stable by } \otimes \mathscr{M} \text {. }
$$

Then $\operatorname{MHM}(X)_{e p}$ is an abelian full subcategory and stable by the natural functors as above by their commutativity with $₫ \mathscr{M}$. In particular, we have

$$
\operatorname{MHM}(p t)=\operatorname{MHM}(p t)_{e p},
$$

because the Hodge Modules (or structures) are stable by the external product with the Hodge structures. By the same argument as in (3.c), we can show in general:

$$
\operatorname{MHM}(X)=\operatorname{MHM}(X)_{e p}
$$

4.3. Theorem. Let $f: X \rightarrow Y$ be a morphism of algebraic varieties. Then we have the functors

$$
f_{!}, f_{*}: D^{b} \operatorname{MHM}(X) \rightarrow D^{b} \operatorname{MHM}(Y)
$$

compatible with the functors on the underlying $\mathbf{Q}$-complexes.

Proof. We repeat the argument in $[1, \S 3]$ using (4.2-8), (4.2.11), i.e. if $X, Y$ are affine, they are the right or left derived functors of $\mathscr{H}^{0} f_{!}, \mathscr{H}^{0} f_{*}$, and in general we reduce to this case using affine open coverings of $X, Y$ compatible with $f$, because we can take a functorial resolution of a finite number of given objects in $\operatorname{MHM}(X)$ simultaneously such that each component is acyclic for the direct images $\left(f_{I}\right)_{!}$(or $\left.\left(f_{I}\right)_{*}\right)$, where $f_{I}$ are the restrictions of $f$ to the intersections of the affine open sets of the coverings, cf. [1,(3.3)]. We can check the independence of covering by taking the covering whose open sets are the open sets of two coverings.

As a corollary of the proof, we have a canonical isomorphism in $D^{b} \operatorname{MHM}(Y)$ :

$$
f_{!}\left(j_{!} \mathscr{M}\right) \simeq j_{!}^{\prime} \mathscr{H}^{0} f_{!}^{\prime} \mathscr{M}\left(\text { resp. } f_{*}\left(j_{*} \mathscr{M}\right) \simeq j_{*}^{\prime} \mathscr{H}^{0} f_{*}^{\prime} \mathscr{M}\right)
$$

for affine open subsets $j: U \rightarrow X$ and $j^{\prime}: U^{\prime} \rightarrow Y$ with $f^{\prime}: U \rightarrow U^{\prime}$ such that $f j$ $=j^{\prime} f^{\prime}$ and for $\mathscr{M} \in \operatorname{MHM}(U)$ whose underlying perverse sheaf is ${ }^{p} \mathscr{H}^{0} f_{!}^{\prime}\left(\right.$ resp. ${ }^{p} \mathscr{H}^{0} f_{*}^{\prime}$ )-acyclic. 
In fact, if $X=U_{i>0} U_{i}$ and $Y=U_{i>0} U_{i}^{\prime}$ are affine open coverings such that $f\left(U_{i}\right) \subset U_{i}^{\prime}$, so are $X=\cup_{i \geq 0} U_{i}, Y=\cup_{i \geq 0} U_{i}^{\prime}$, where $U_{0}^{(\prime)}=U^{(\prime)}$. Then $j_{!}^{\prime} \mathscr{H}^{0} f_{!}^{\prime} \mathscr{M}$ is a subcomplex of the Čch complex, associated to the second coverings, whose components are the direct images $\left(j_{I}^{\prime}\right)_{!} \mathscr{H}^{0}\left(f_{I}\right)_{!} j_{I}^{-1} j_{!} \mathscr{M}$, where $f_{I}: U_{I} \rightarrow U_{I}^{\prime}$ and $j_{I}^{\prime}: U_{I}^{\prime} \rightarrow Y$. Therefore, taking a resolution of $j_{!} \mathscr{M}$ as above, we get the morphism $j_{!}^{\prime} \mathscr{H}^{0} f_{!}^{\prime} \mathscr{M} \rightarrow f_{!}\left(j_{!} \mathscr{M}\right)$, which is an isomorphism, because it is true for the underlying $\mathbb{Q}$-complexes.

By (4.3.1) we have the natural isomorphisms:

$$
(g f)_{!}=g_{!} f_{!}, \quad(g f)_{*}=g_{*} f_{*}
$$

for $f: X \rightarrow Y, g: Y \rightarrow Z$, because we can take a resolution such that each component is $\mathscr{H}^{0}\left(f_{I}\right)_{\text {! }}$-acyclic and $\mathscr{H}^{0}\left(g_{I} f_{I}\right)$ !-acyclic at the same time by the construction in $[1,(3.3)]$.

We can also show the canonical isomorphism

$$
f_{!}=f_{*}
$$

if $f$ proper. In fact it is enough to show the isomorphism

$$
f_{!}\left(j_{!} \mathscr{M}\right)=f_{*}\left(j_{!} \mathscr{M}\right) \text { in } \operatorname{MHM}(Y)
$$

compatible with the canonical isomorphism on the underlying perverse sheaves for $j: U \rightarrow X$ and $\mathscr{M}$ as in (4.3.1), because every objects of $D^{b} \mathrm{MHM}(X)$ is isomorphic to a complex (in $D^{+} \operatorname{MHM}(X)$ ) whose components are of the above type (by the proof of 4.3). Let $\hat{j}: U \rightarrow \tilde{X}$ be an open immersion and $\pi: \tilde{X} \rightarrow X$ a proper morphism such that $\tilde{X}$ is quasi-projective and $\pi \tilde{j}=j$. If the assertion is verified for $\pi$ and $f \pi$, we get

$$
\begin{aligned}
& f_{!} j_{!} \mathscr{M}=f_{!} \pi_{!} \tilde{j}_{!} \mathscr{M}=(f \pi)_{!} \tilde{j}_{!} \mathscr{M} \\
& \quad=(f \pi)_{*} \tilde{j}_{!} \mathscr{M}=f_{*} \pi_{*} \tilde{j}_{!} \mathscr{M}=f_{*} \pi_{!} \tilde{j}_{!} \mathscr{M}=f_{*} j_{!} \mathscr{M}
\end{aligned}
$$

by (4.3.2). Therefore the assertion is reduced to the case $X, Y$ projective and follows from the next assertion:

For $f: X \rightarrow Y$ a morphism of projective varieties and $\mathscr{M} \in \mathbb{M H M}(X)$, we have the canonical isomorphism in $\operatorname{MHM}(Y)$ :

$$
H^{j}\left(f_{!} \mathscr{M}\right)=\left(\mathscr{H}^{j} f_{*}\right) \mathscr{M}=H^{j}\left(f_{*} \mathscr{M}\right)
$$

compatible with the canonical isomorphisms on the underlying perverse sheaves. (Here $\mathscr{H}^{j} f_{*}$ is defined in (4.2.8).)

By the definition of $f_{!}, f_{*}$ and by (4.3.1), it is enough to show that $\left\{\mathscr{H}^{j} f_{*}\right\}$ is extended to a cohomological functor from $D^{b} \operatorname{MHM}(X)$ to $\operatorname{MHM}(Y)$. But this is verified by the same argument as in $2.14-16$, because the $\mathbb{Q}$-part of an object of $D^{b} M H M(X)$ defines a quasi-filtered object of $D_{c}^{b}\left(\mathbb{Q}_{X}\right)(\mathrm{cf} .[19,(5.2 .17)])$ by the functor real defined in $[3,3.1 .10]$ so that the weight spectral sequence is well- 
defined as in the proof of 2.15 (here the weight filtration on $X$ is shifted by the degree of complex so that $H^{j} \mathrm{Gr}_{i}^{W}$ is of weight $i+j$ ).

Similarly we can show

$$
\mathbb{D} f_{!}=f_{*} \mathbf{D}
$$

In fact, it is enough to show for $j_{!} \mathscr{M}$ as above. Because we have

$$
\begin{aligned}
& \mathbf{D} f_{!} j_{!} \mathscr{M}=\mathbf{D} j_{!}^{\prime}\left(\mathscr{H}^{0} f_{!}^{\prime}\right) \mathscr{M}=j_{*}^{\prime} \mathbf{D}\left(\mathscr{H}^{0} f_{!}^{\prime}\right) \mathscr{M} \\
& f_{*} \mathbf{D} j_{!} \mathscr{M}=f_{*} j_{*} \mathbb{D} \mathscr{M}=j_{*}^{\prime}\left(\mathscr{H}^{0} f_{*}^{\prime}\right) \mathbf{D} \mathscr{M}
\end{aligned}
$$

(where $j^{\prime}, f^{\prime}$ are as in (4.3.1)), it is enough to show

$$
\mathrm{D}\left(\mathscr{H}^{j} f_{!}\right) \mathscr{M}=\left(\mathscr{H}^{-j} f_{*}\right) \mathbf{D} \mathscr{M}
$$

in the case $X, Y$ affine, or in the case $X, Y$ smooth projective by definition. Then this follows from the compatibility of the algebraic and topological dualities, because the weight spectral sequence is compatible with the duality. Here the algebraic duality is easily verified in the case $f=\operatorname{pr}_{2}: X=\mathbb{P}^{n}$ $\times Y \rightarrow Y$, because the trace morphism is easily defined (and compatible with the topological one).

4.4. Let $f: X \rightarrow Y$ be a morphism of algebraic varieties. We define $f^{*}$ (resp. $f^{!}$) by the left (resp. right) adjoint functor of $f_{*}$ (resp. $f_{!}$). Then $\mathbf{D} f^{!}=f^{*} \mathbf{D}$ by (4.3.5). Let $g: Y \rightarrow Z$ be a morphism of algebraic varieties. Then $(g f)^{*}$ exists and $(g f)^{*} \simeq f^{*} g^{*}$ if $f^{*}$ and $g^{*}$ exist by (4.3.2) (same for $(g f)^{\prime}$ ). We show the existence of $f^{*}, f^{!}$and their compatibility with the functors on the underlying $\mathbf{Q}$ complexes using the factorization $f: X \rightarrow X \times Y \rightarrow Y$.

(4.4.1) Let $i: X \rightarrow Y$ be a closed immersion, and $j: U \rightarrow Y$ the immersion of the complement. Then $j^{!}=j^{*}=j^{-1}$, and $i^{*}$ (resp. $i^{!}$) exists and is compatible with $\mathbf{Q}$-structure. We have the functorial triangles for $\mathscr{M} \in D^{b} \operatorname{MHM}(Y)$ :

$$
\begin{gathered}
\longrightarrow j_{!} j^{-1} \mathscr{M} \longrightarrow \mathscr{M} \longrightarrow i_{*} i^{*} \mathscr{M} \stackrel{+1}{\longrightarrow} \\
\text { (resp. } \longrightarrow i_{*} i ! \mathscr{M} \longrightarrow \mathscr{M} \longrightarrow j_{*} j^{-1} \mathscr{M} \stackrel{+1}{\longrightarrow} \text { ) }
\end{gathered}
$$

compatible with those for the underlying $\mathbf{Q}$-complexes.

This follows from the same argument as in 2.20. We take an affine open covering of $U$ and use the associated Čech complex (with (4.2. 10-11)) to define the triangles and $i^{*}, i^{!}$. Then the first assertion follows from the existence of morphisms $j_{!} j^{-1} \rightarrow \mathrm{id} \rightarrow j_{*} j^{-1}$ (in the triangles), and it implies 


$$
\operatorname{Hom}\left(j_{!} j^{-1} \mathscr{M}, i_{*} \mathcal{N}\right)=\operatorname{Hom}\left(i_{*} \mathcal{N}, j_{*} j^{-1} \mathscr{M}\right)=0 .
$$

Thus we get the adjunctions by the triangles.

For a projection $p: X \times Y \rightarrow Y, p^{*}$ (resp. $p^{!}$) exists and is represented by $\mathscr{M} \otimes$ for any $\mathscr{M} \in D^{b} \operatorname{MHM}(X)_{e p}$ (cf. (4.2.13)) with a morphism in $D^{b} \mathbf{M H M}(p t): \mathbf{Q}_{p t}^{H} \rightarrow\left(a_{X}\right)_{*} \mathscr{M}$ (resp. $\left(a_{X}\right)_{!} \mathscr{M} \rightarrow \mathbf{Q}_{p t}^{H}$ ) such that $a_{X}^{*} \mathbf{Q}$ (resp. $a_{X}^{!} \mathbf{Q}$ ) underlies $\mathscr{M}$ and the natural morphism $\mathbb{Q} \rightarrow\left(a_{X}\right)_{*} a_{X}^{*} \mathbb{Q}$ (resp. $\left.\left(a_{X}\right) ! a_{X}^{!} \mathbf{Q} \rightarrow \mathbf{Q}\right)$ underlies the above morphism. In particular, $\mathscr{M}$ $\simeq a_{X}^{*} \mathbf{Q}_{p t}^{H}$ (resp. $\left.a_{X}^{!} \mathbf{Q}_{p t}^{H}\right)$, and if $X$ smooth, $\mathscr{M} \simeq \mathbf{Q}_{X}^{H}$ (resp. $\mathbf{Q}_{X}^{H}\left(d_{X}\right)\left[2 d_{X}\right]$ ).

Here $a_{X}: X \rightarrow p t=\operatorname{Spec} C$, and $\mathbb{Q}_{X}^{H}\left[d_{X}\right]=\left(\left(\Omega_{X}^{d_{X}}, F\right), \mathbb{Q}_{X}\left[d_{X}\right] ; W\right)$ with $\operatorname{Gr}_{i}^{W}$ $=\mathrm{Gr}_{-i}^{F}=0$ for $i \neq d_{X}$ (if $X$ smooth).

We prove the assertion for $p^{*}$, because the argument is dual for $p^{!}$. We have to construct the functorial morphisms

$$
\alpha: \text { id } \longrightarrow p_{*} p^{*}, \quad \beta: p^{*} p_{*} \rightarrow \text { id }
$$

such that the compositions

$$
\begin{aligned}
& \beta \circ p^{*} \alpha: p^{*} \mathcal{N} \longrightarrow p^{*} p_{*} p^{*} \mathcal{N} \longrightarrow p^{*} \mathcal{N} \\
& p_{*} \beta \circ \alpha: p_{*} \mathscr{N}^{\prime} \longrightarrow p_{*} p^{*} p_{*} \mathcal{N}^{\prime} \longrightarrow p_{*} \mathcal{N}^{\prime}
\end{aligned}
$$

are the identities. We define $\alpha$ by the external product of $\mathbf{Q}_{p t}^{H} \rightarrow\left(a_{X}\right)_{*} \mathscr{M}$. For $\beta$ we use the diagram:

(*)



where $i$ is the diagonal embedding such that $q_{j}{ }^{\circ} i=\mathrm{id}$. We define $\beta$ by the composition:

$$
\begin{aligned}
& p_{2}^{*}\left(p_{1}\right)_{*} \mathscr{N}^{\prime}=\mathscr{M} \bigotimes\left(p_{1}\right)_{*} \mathcal{N}^{\prime}=\left(q_{2}\right)_{*}\left(\mathscr{M} \bowtie \mathscr{N}^{\prime}\right) \\
& \left(=\left(q_{2}\right)_{*} q_{1}^{*} \mathcal{N}^{\prime}\right) \longrightarrow\left(q_{2}\right)_{*} i_{*} i^{*}\left(M 区 \mathscr{N}^{\prime}\right) \simeq \mathscr{N}^{\prime},
\end{aligned}
$$

where the last isomorphism is induced by (4.3.2) and the following assertion (applied to $i$ and $q_{1}$ ):

Let $i: Y \rightarrow X \times Y$ be a section of the projection $p: X \times Y \rightarrow Y$, and $\mathscr{M}$ as in (4.4.2). Then the composition:

$$
N \stackrel{\alpha}{\longrightarrow} p_{*}(\mathscr{M} \bowtie \mathcal{N}) \longrightarrow p_{*} i_{*} i^{*}(\mathscr{M} \bowtie \mathscr{N})=i^{*}(\mathscr{M} \bowtie \mathscr{N})
$$

is an isomorphism. (In fact, it is true for the underlying $\mathbf{Q}$-complexes.)

Then $p_{*} \beta \circ \alpha=\mathrm{id}$ is clear, and $\beta \circ p^{*} \alpha=\mathrm{id}$ follows from the symmetry of the 
diagram, i.e. the isomorphism: $i^{*}(\mathscr{M} \otimes \mathscr{M} \otimes \mathscr{N}) \simeq \mathscr{M} \otimes \mathscr{N}$ used in the definition of $\beta$ is independent of the choice of the projection onto $X \times Y$.

Here note that the above argument and the arguments in 4.3 and (4.4.1) hold with $\operatorname{MHM}(X)$ replaced by $\operatorname{MHM}(X)_{e p}$ (cf. (4.2.13), here we can also use (4.2.15)). We show the existence of $\mathscr{M}$ satisfying the conditions in (4.4.2). If $X$ is smooth, $\mathscr{M}=\mathbf{Q}_{X}^{H}\left[d_{X}\right]=\mathscr{H}^{d_{X}} a_{X}^{*} \mathbf{Q}_{p t}^{H}$ belongs to $\operatorname{MHM}(X)_{e p}$ by (4.2.14). By (4.4.1) for $\operatorname{MHM}(X)_{e p}$, the assertion is verified locally, and it is enough to show the following:

If $X$ is covered by two open sets $U_{i}$ such that $\mathscr{M}_{i}$ exists on $U_{i}(i=1,2), \mathscr{M}$ exists on $X$.

Put $U_{3}=U_{1} \cap U_{2}$ and let $j_{i}: U_{i} \rightarrow X$ be the natural inclusions. By the adjunction for $U_{3} \rightarrow U_{i}$ and $U_{i} \rightarrow p t, \quad \mathscr{M}_{3} \simeq a_{U_{3}}^{*} \mathbf{Q}_{p t}^{H}$ exists on $U_{3}$ (in $\left.D^{b} \operatorname{MHM}\left(U_{3}\right)_{e p}\right)$ and we have the isomorphism $\left.\mathscr{M}_{3} \simeq \mathscr{M}_{i}\right|_{U_{3}}$ such that $\mathbb{Q}_{p t}^{H}$ $\rightarrow\left(a_{U_{3}}\right)_{*} \mathscr{M}_{3}$ is given by the composition:

$$
\mathbb{Q}_{p t}^{H} \rightarrow\left(a_{U_{1}}\right)_{*} \mathscr{M}_{i} \longrightarrow\left(a_{U_{3}}\right)_{*} \mathscr{M}_{3}
$$

for $i=1,2$, because the adjunction isomorphism for $a_{X}$ is give by $\alpha$. We define $\mathscr{M}$ by the mapping cone:

$$
C\left(\oplus_{i=1,2}\left(j_{i}\right)_{*} \mathscr{M}_{i} \longrightarrow\left(j_{3}\right)_{*} \mathscr{M}_{3}\right)[-1]
$$

so that we have the exact sequence

$$
0 \longrightarrow H^{0}\left(a_{X}\right)_{*} \mathscr{M} \longrightarrow \oplus H^{0}\left(a_{U_{1}}\right)_{*} \mathscr{M}_{i} \rightarrow H^{0}\left(a_{U_{3}}\right)_{*} \mathscr{M}_{3} .
$$

Then the assertion is clear, because $H^{i}\left(a_{X}\right)_{*} \mathscr{M}=0$ for $i<0$.

Remark. The above construction of $\beta$ is due to Kashiwara, i.e. the use of the diagram $(*)$ is suggested by him.

As a corollary of the above argument, we have

(4.4.3) For a cartesian diagram

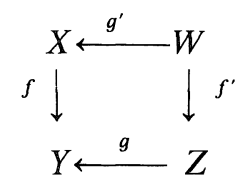

we have the canonical isomorphisms:

$$
g^{!} f_{*} \simeq f_{*}^{\prime}\left(g^{\prime}\right)^{!}, \quad g^{*} f_{!} \simeq f_{!}^{\prime}\left(g^{\prime}\right)^{*}
$$

compatible with the natural isomorphism on the underlying $\mathbf{Q}$ complexes.

In fact, we may assume $g$ is a closed immersion or a projection. The projection 
case is clear by definition. In the closed immersion case, we take affine open coverings $X=\cup_{i \in I} U_{i}^{\prime}, Y=\cup_{i \in I} U_{i}$ compatible with $f$ and satisfying $U^{\prime}=\cup_{i \in J} U_{i}^{\prime}$, $U=\cup_{i \in J} U_{i}$ for $J \subset I$, where $j^{\prime}: U^{\prime}=X \backslash W \rightarrow X, j: U=Y \backslash Z \rightarrow Y$. We take a resolution of $M \in C^{b} \mathrm{MHM}(X)$ compatible with these coverings to define $f_{*} \mathscr{M}$. Then its quotient complex whose components are the direct images from $\cap_{i \in K} U_{i}^{\prime}$ with $K \subset J$ represents $f_{*} j_{*}^{\prime}\left(j^{\prime}\right)^{-1} \mathscr{M}$, and the mapping cone of the quotient morphism represents $g_{*} f_{*}^{\prime}\left(g^{\prime}\right)^{!} \mathscr{M}=f_{*} g_{*}^{\prime}\left(g^{\prime}\right)^{!} \mathscr{M}$. We verify that this quotient morphism also represents the morphism, $f_{*} \mathscr{M} \rightarrow j_{*} j^{-1} f_{*} \mathscr{M}$, applying id $\rightarrow j_{*} j^{-1}$ to this morphism, where $j_{*} j^{-1}$ is also defined in the level of complex using a covering. Then we get the assertion.

4.5. We say that $\mathscr{M} \in D^{b} \operatorname{MHM}(X)$ is of weight $\leq n$ (resp. $\geq n$ ), if the following condition is satisfied:

$$
\mathrm{Gr}_{i}^{W} H^{j} \mathscr{M}=0 \text { for } i>n+j \text { (resp. } i<n+j \text { ). }
$$

By 2.26 and (4.3.4), we can verify:

(4.5.2) the condition (4.5.1) is stable by the functors $f_{!}, f^{*}$ (resp. $f_{*}, f^{!}$).

We say that $\mathscr{M} \in D^{b} \mathrm{MHM}(X)$ is pure of weight $n$, if $\mathrm{Gr}_{i}^{W} H^{j} \mathscr{M}=0$ for $i \neq j$ $+n$. By (4.5.2) this condition is stable by $f_{*}$ if $f$ is proper. (Actually the last assertion is first proved in the proof of (4.5.2) for direct images, where we use Chow's lemma and the decomposition (4.5.4) below.) We can also verify

$$
\operatorname{Ext}^{j}(\mathscr{M}, \mathscr{N})=0 \text { for } \mathscr{M}, \mathscr{N} \text { pure of weight } m, n \text { such that } m<n+j,
$$

using Yoneda's extension, because $\operatorname{MH}(X, n)^{p}$ are semi-simple and $\operatorname{MHM}(X)$ is stable by sub-quotients in $\operatorname{MHW}(X)$ (or we can use $\operatorname{MH}(X, n)^{p} \subset \operatorname{MHM}(X)$, which follows from the algebraic version of 3.27). As a corollary, we get a non canonical isomorphism in $D^{b} \operatorname{MHM}(X)$ :

$$
\mathscr{M} \cong \oplus H^{j} \mathscr{M}[-j]
$$

if $\mathscr{M}$ is pure of weight $n$. (These facts are analogue of [3].)

For an algebraic variety $X$, we define

$$
\mathbb{Q}_{X}^{H}:=a_{X}^{*} \mathbf{Q}_{p t}^{H}
$$

where $a_{X}: X \rightarrow p t(:=\operatorname{Scec} \mathbb{C})$ and $\mathbb{Q}_{p t}^{H}:=(\mathbb{C}, F, \mathbb{Q} ; W) \in \operatorname{MHM}(p t)$ with $\operatorname{Gr}_{i}^{F}$ $=\mathrm{Gr}_{i}^{W}=0$ for $i \neq 0$, cf. 3.8. Then

$$
H^{i} \mathbf{Q}_{X}^{H}=0 \text { for } i>d_{X}(:=\operatorname{dim} X),
$$

because the functor rat is faithful and ${ }^{p} \mathscr{H}^{i}\left(Q_{X}\left[d_{X}\right]\right)=0$ for $i>0$ by definition. By (4.5.2), $\mathbb{Q}_{X}^{H}$ is of weight $\leq 0$; in particular we get

$$
\mathrm{Gr}_{i}^{W} H^{d_{X}} \mathrm{Q}_{X}^{H}=0 \text { for } i>d_{X} .
$$


By (4.5.6) we have also

$$
\operatorname{Hom}\left(\mathbf{Q}_{X}^{H}\left[d_{X}\right], \mathscr{M}\right)=\operatorname{Hom}\left(H^{0}\left(\mathbf{Q}_{X}^{H}\left[d_{X}\right]\right), \mathscr{M}\right)=0
$$

for $\mathscr{M} \in \operatorname{MHM}(X)$ such that $\operatorname{dim} \operatorname{supp} \mathscr{M}<d_{X}$, because these groups are isomorphic to $\operatorname{Hom}\left(\mathbf{Q}_{Z}^{H}\left[d_{X}\right], \mathscr{M}\right)$ for $Z=\operatorname{supp} \mathscr{M}$ by adjunction. Let $U$ be the maximal smooth open subset of $X$ of pure dimension $d_{X}$, and $X^{\prime}$ the closure of $U$ in $X$. Then (4.5.8) implies

$$
\mathrm{Gr}_{d_{X}}^{W} H^{d_{X}} \mathbf{Q}_{X}^{H}=\mathrm{IC}_{X^{\prime}} \mathbf{Q}^{H},
$$

because $\operatorname{MH}\left(X, d_{X}\right)^{p}(\subset \operatorname{MHM}(X))$ is semi-simple. Here $\operatorname{IC}_{X^{\prime}} \mathbf{Q}^{H}$ is a unique object of $\operatorname{MHM}(X)$ such that its restriction to $U$ is $\mathbf{Q}_{U}^{H}\left[d_{X}\right]$ and it has no subobject and no quotient object in $\operatorname{MHM}(X)$, whose support is contained in $X \backslash U$. Then $\mathrm{IC}_{X^{\prime}} \mathbf{Q}^{H}$ is simple and pure of weight $d_{X}$, because so is $\mathbf{Q}_{U}^{H}\left[d_{X}\right]$ by definition. For the uniqueness of $\mathrm{IC}_{X^{\prime}} \mathbf{Q}^{H}$, we have the natural isomorphism (cf. [3]):

$$
\mathrm{IC}_{X^{\prime}} \mathbf{Q}^{H}=\operatorname{Im}\left(H^{0} j_{!}\left(\mathbf{Q}_{U}^{H}\left[d_{X}\right]\right) \rightarrow H^{0} j_{*}\left(\mathbf{Q}_{U}^{H}\left[d_{X}\right]\right)\right)
$$

where $j: U \rightarrow X$. In fact we have the adjunction morphism

$$
H^{0} j_{!} j^{-1} \mathscr{M} \longrightarrow \mathscr{M} \text { (resp. } \mathscr{M} \longrightarrow H^{0} j_{*} j^{-1} \mathscr{M} \text { ) }
$$

for $\mathscr{M}=\mathrm{IC}_{X^{\prime}} \mathbf{Q}^{H}$ by (4.4.1), because $H^{i} j_{!} j^{-1} \mathscr{M}=0(i>0)$ (resp. $H^{i} j_{*} j^{-1} \mathscr{M}$ $=0(i<0))$ for $\mathscr{M} \in \operatorname{MHM}(X)$ by definition. Then the surjectivity (resp. injectivity) of the adjunction morphism is clear, and we get (4.5.10).

As a corollary of (4.5.6-7) and (4.5.9), we get the natural (quotient) morphism in $D^{b} \operatorname{MHM}(X)$ :

$$
\mathbf{Q}_{X}^{H} \rightarrow \mathrm{IC}_{X^{\prime}} \mathbf{Q}^{H}\left[-d_{X}\right]
$$

Taking the composition with its dual, we get the natural morphism in $D^{b} \operatorname{MHM}(X)$ :

$$
\mathbf{Q}_{X}^{H} \rightarrow\left(\mathbb{D} \mathbf{Q}_{X}^{H}\right)\left(-d_{X}\right)\left[-2 d_{X}\right]
$$

because we have the self-duality:

$$
\mathbf{D}\left(\mathrm{IC}_{X^{\prime}} \mathbf{Q}^{H}\right)=\mathrm{IC}_{X^{\prime}} \mathbf{Q}^{H}\left(d_{X}\right)
$$

(In fact (4.5.13) is trivial on $U$ by definition.) By (4.5.8) and its dual, we get

$$
\operatorname{Hom}\left(\mathbf{Q}_{X}^{H},\left(\mathbf{D} \mathbf{Q}_{X}^{H}\right)\left(-d_{X}\right)\left[-2 d_{X}\right]\right)=\operatorname{End}\left(\mathrm{IC}_{X^{\prime}} \mathbf{Q}^{H}\right)=\mathbf{Q}^{r}
$$

where $r$ is the number of the irreducible components of $U$. In particular the morphism (4.5.12) is uniquely determined by its restriction to an open dense subset of $U$.

Let $Z$ be a closed subvariety of dimension $d$ of $X$. We apply (4.5.12) to 
$Z$. Taking its composition with the adjunction morphism $\mathbb{Q}_{X}^{H} \rightarrow \mathbb{Q}_{Z}^{H}$ and its dual, we get a morphism in $D^{b} \operatorname{MHM}(X)$ :

$$
\mathbb{Q}_{X}^{H} \rightarrow\left(\mathbb{D Q}_{X}^{H}\right)(-d)[-2 d] .
$$

By definition its restriction to $X \backslash Z$ is zero, and we get a factorization:

$$
\mathbb{Q}_{X}^{H} \rightarrow \mathbb{Q}_{Z}^{H} \longrightarrow\left(\mathbb{D} \mathbb{Q}_{Z}^{H}\right)(-d)[-2 d] \rightarrow\left(\mathbb{D} \mathbb{Q}_{X}^{H}\right)(-d)[-2 d],
$$

which is unique if $Z$ is irreducible.

Assume now $Z$ is irreducible, and let $\pi: \tilde{Z} \rightarrow Z$ be a resolution of singularity . Then the morphism (4.5.15) is the composition of the natural morphism:

$$
\mathbb{Q}_{X}^{H} \rightarrow \mathbb{Q}_{Z}^{H} \rightarrow \pi_{*} \mathbb{Q}_{Z}^{H}
$$

(induced by the adjunction) and its dual, because the middle morphism of $(4,5.16)$


morphism (4.5.15) corresponds to an element $c l_{Z}^{H}$ of

$$
\operatorname{Hom}\left(\mathbb{Q}_{p t}^{H},\left(a_{X}\right)_{*}\left(\mathbb{D} \mathbb{Q}_{X}^{H}\right)(-d)[-2 d]\right),
$$

which is equal to the composition:

$$
\begin{aligned}
\mathbb{Q}_{p t}^{H} \stackrel{\sim}{\rightarrow} H^{0}\left(a_{Z}\right)_{*} \mathbb{Q}_{Z}^{H} \stackrel{\sim}{\longrightarrow} & H^{0}\left(\left(a_{Z}\right)_{*} \operatorname{IC}_{Z} \mathbb{Q}^{H}[-d]\right) \stackrel{\sim}{\rightarrow} H^{0}\left(\left(a_{Z}\right)_{*}\left(\mathbb{D} \mathbb{Q}_{Z}^{H}\right)(-d)[-2 d]\right) \\
& \left.\left.\longrightarrow\left(a_{Z}\right)_{*}\left(\mathbb{D} \mathbf{Q}_{Z}^{H}\right)(-d)[-2 d]\right) \longrightarrow\left(a_{X}\right)_{*}\left(\mathbb{D} \mathbb{Q}_{X}^{H}\right)(-d)[-2 d]\right)
\end{aligned}
$$

and called the (Hodge) cycle class of $Z$. If $X$ is smooth, we have the self duality $\mathbb{D} \mathbb{Q}_{X}^{H}=\mathbb{Q}_{X}^{H}\left(d_{X}\right)\left[2 d_{X}\right]$ by (4.5.13), and (4.5.17) is isomorphic to the $\mathbb{Q}$-Deligne cohomology:

$$
\operatorname{Hom}\left(\mathbb{Q}_{p t}^{H},\left(a_{X}\right)_{*} \mathbb{Q}_{X}^{H}(p)[2 p]\right),
$$

if $X$ is smooth and proper (cf. [2]), where $p=\operatorname{codim} Z$. Note that $H^{\cdot}\left(a_{X}\right)_{*} \mathbf{D} \mathbb{Q}_{X}^{H}$ gives the mixed Hodge structure on the Borel-Moore homology of $X$ in general. We can show that the above construction induces the cycle map:

$$
\mathrm{CH}_{d}(X) \otimes \mathbb{Q} \rightarrow \operatorname{Hom}\left(\mathbb{Q}_{p t}^{H},\left(a_{X}\right)_{*} a_{X} \mathbb{Q}_{p t}^{H}(-d)[-2 d]\right),
$$

where $\mathbb{D} \mathbb{Q}_{X}^{H}=a_{X}^{!} \mathbb{Q}_{p t}^{H}$ by 4.4. Let $\tilde{Z}$ be a cycle of dimension $d+1$ on $Y=X$ $\times \mathbb{P}^{1}$, and $Z_{t}$ its intersection with $X \times\{t\}$ for $t \in \mathbb{P}^{1}$. Then $\tilde{Z}$ determines an element of

$$
\operatorname{Hom}\left(\mathbb{Q}_{Y}^{H}, \pi^{!} \mathbb{Q}_{S}^{H}(-d)[-2 d]\right)
$$

by the above construction (taking the sum of the cycle class of each irreducible component, multiplied by the coefficient), where $S=\mathbb{P}^{1}$ and $\pi: Y \rightarrow S$ is the second projection. For each $t \in \mathbb{P}^{1}$, we can take the vanishing cycle functor $\psi$ along $X \times\{t\}$, and get an element $c l_{t}^{\prime}$ of 


$$
\operatorname{Hom}\left(\mathbf{Q}_{X}^{H}, a_{X}^{!} \mathbf{Q}_{p t}^{H}(-d)[-2 d]\right),
$$

because $\psi$ is an exact functor, and $\pi^{!}$is defined by $\bigotimes a_{X}^{!} \mathbf{Q}_{p t}^{H}$ which commutes with the vanishing cycle functor (same for $\mathbf{Q}_{Y}^{H}=\pi^{*} \mathbf{Q}_{S}^{H}$ ). By definition of $Z_{t}$ and $c l_{t}^{\prime}$ we can check that $c l_{t}^{\prime}$ coincides with the cycle class of $Z_{t}$, using the factorization by $\mathbf{Q}_{Y}^{H} \rightarrow \mathbf{Q}_{|Z|}^{H}$, the natural morphism of functors $i^{*} \rightarrow \psi[1]$ (cf. 2.24) and their duals, and observing at the generic point of $\left|Z_{t}\right|$, where $|\tilde{Z}|$ and $\left|Z_{t}\right|$ are the support of $\tilde{Z}$ and $Z_{t}$. Then we see that the adjunction isomorphisms are compatible with the vanishing cycle functor, because it is induced by the external product with $\mathbf{Q}_{p t}^{H} \rightarrow\left(a_{X}\right)_{*} \mathbf{Q}_{X}^{H}$, cf. the proof of (4.4.2). Therefore it is enough to check the following:

$$
\begin{aligned}
& \text { for } \mathscr{M} \in D^{b} \operatorname{MHM}(p t) \text { and } \xi \in \operatorname{Hom}\left(\mathbf{Q}_{S}^{H}, \mathbf{Q}_{S}^{H} \otimes \mathscr{M}\right), \psi_{t} \xi \in \operatorname{Hom}\left(\mathbf{Q}_{p t}^{H}, \mathscr{M}\right) \\
& \text { the vanishing cycle of } \xi \text { along } t \in S=\mathbf{P}^{1} \text { is independent of } t \text {. }
\end{aligned}
$$

By [2] we have $\operatorname{Ext}^{i}(\mathscr{M}, \mathscr{N})=0(i \geq 2)$ for $\mathscr{M}, \mathscr{N} \in \operatorname{MHM}(p t)$, and it is enough to show the assertion for $\mathscr{M}$ and $\mathscr{M}[1]$ with $\mathscr{M} \in \mathrm{MHM}(p t)$. Then the assertion for $\mathscr{M}$ is clear, and that for $\mathscr{M}[1]$ follows from 2.27 (applying it to the variation of mixed Hodge structure corresponding to the extension class), because $S$ is simply connected. This completes the proof of (4.5.19) and the well-definedness of (4.5.18). Next we show:

the morphism (4.5.18) induces the Abel-Jacobi morphism of Griffiths, tensored by $\mathbf{Q}$, if $X$ is smooth and proper.

By Deligne, Griffiths' Abel-Jacobi map is expressed as follows: Let $Z$ be a cycle on $X$, homologous to zero. Then we have an exact sequence in $\mathrm{MHM}(p t)$ :

$$
0 \longrightarrow H^{2 p-1}(X, \mathbf{Q}) \rightarrow H^{2 p-1}(U, \mathbf{Q}) \rightarrow H_{|Z|}^{2 p}(X, \mathbf{Q})_{0} \longrightarrow 0
$$

where $|Z|$ is the support of $Z, \quad U=X \backslash Z \mid, \quad p=\operatorname{codim} Z$ and $H_{|Z|}^{2 p}(X)_{0}$ $=\operatorname{Ker}\left(H_{|Z|}^{2 p}(X) \rightarrow H^{2 p}(X)\right)$. Here $H_{|Z|}^{2 p}(X)$ and $H_{|Z|}^{2 p}(X)_{0}$ are pure of type $(p, p)$, and the image of Griffiths' Abel-Jacobi map of $Z$ in the intermediate Jacobian tensored by $\mathbf{Q}$ corresponds to the extension class defined by the pull-back of the above exact sequence by the morphism

$$
\mathbf{Q}(-p) \longrightarrow H_{|Z|}^{2 p}(X, \mathbf{Q})_{0}\left(\subset H_{|Z|}^{2 p}(X, \mathbf{Q})=\oplus \mathbf{Q}(-p)\right)
$$

which is defined by (the coefficients of) $Z$, i.e. we replace $H^{2 p-1}(U, \mathbf{Q})$ in the exact sequence by the kernel of the natural morphism onto the cokernel of $\mathbf{Q}(-p) \rightarrow H_{|Z|}^{2 p}(X, \mathbf{Q})_{0}$, using the diagram of the nine lemma. Then we have to show that the above construction gives the same extension class. But it is clear by a morphism of triangles: 


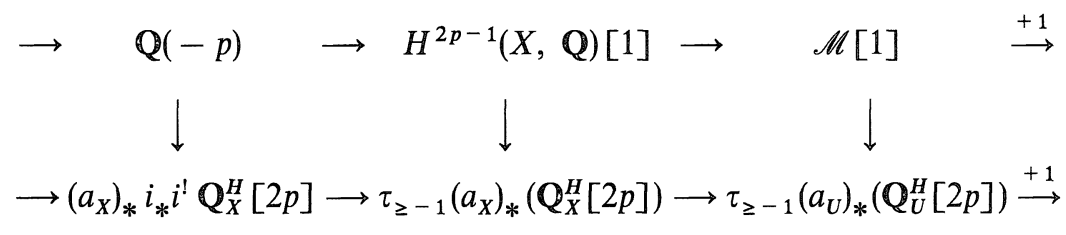

Here the composition $\mathbb{Q}(-p) \rightarrow H_{|Z|}^{2 p}(X, \mathbb{Q}) \rightarrow\left(a_{X}\right)_{*} \mathbb{Q}_{X}^{H}[2 p]$ coincides with $c l_{Z}^{H}$ by definition, where

$$
\begin{aligned}
H_{|Z|}^{2 p}(X, \mathbb{Q}(p)) & =H^{0}\left(a_{|Z|}\right)_{*} a_{|Z|}^{\prime} \mathbb{Q}^{H}\left(-d_{Z}\right)\left[-2 d_{Z}\right] \\
& =H^{0}\left(a_{X}\right)_{*} i_{*} i^{!}\left(\mathbb{Q}_{X}^{H}(p)[2 p]\right)
\end{aligned}
$$

using $\mathbb{R} \underline{\Gamma}_{|Z|}=i_{*} i^{!} \quad$ with $i:|Z| \rightarrow X$, and $a_{X}^{!} \mathbb{Q}^{H}=\mathbb{Q}_{X}^{H}\left(d_{X}\right)\left[2 d_{X}\right]$. Then the composition is factorized by $\tau_{<0}\left(a_{X}\right)_{*}\left(\mathbf{Q}_{X}^{H}[2 p]\right)$ by assumption, and we apply the functor $\tau_{\geq-1}$.

Remark. In the above argument we used the compatibility between Deligne's mixed Hodge structure [9] and that of 4.3-4. We can easily verify it for the cohomology $H^{\cdot}(Z)$ and the local cohomology $H_{Z}^{\cdot}(X)$ (i.e. the Borel-Moore homology of $Z$ ) in the case $Z$ is a closed subvariety of a smooth variety $X$. In fact, using an embedded resolution of $Z$ and the functoriality of the mixed Hodge structures (with the base change (4.4.3)), we can reduce to the case where $Z$ is a normal crossing divisor. Then the assertion is clear.

4.6. Remarks. 1. For $\mathscr{M} \in D^{b} \operatorname{MHM}(X)$ we have:

$\mathscr{M}$ is of weight $\leq n$ (resp. $\geq n$ ), iff $H^{k} i_{x}^{*} \mathscr{M}$ (resp. $\left.H^{k} i_{x}^{!} \mathscr{M}\right)$ is of weight $\leq n+k$ (resp. $\geq n+k$ ) for any $k$ and $x$.

Here $i_{x}:\{x\} \rightarrow X$, and the condition for $i_{x}^{!}$is equivalent to: $H^{k} i_{x}^{*} \mathbb{M}$ is of weight $\leq-n+k$, by duality. In fact, the assertion follows by induction on the dimension of the support, using the distinguished triangles in (4.4.1) and (4.5.2), because it is clear generically, i.e. for a variation of mixed Hodge structure.

2. We have a $t$-structure $\left({ }^{c} D^{\leq 0},{ }^{c} D^{\geq 0}\right)$ on $D^{b} \operatorname{MHM}(X)$ such that ${ }^{c} D^{\leq 0}\left(\right.$ resp. $\left.{ }^{c} D^{\geq 0}\right)$ is defined by the condition:

(4.6.2) for any closed immersion $i_{S}: S \rightarrow X$, there is a non-emptity open subset $U$ of $S$ such that $\left.H^{k} i_{S}^{*} \mathscr{M}\right|_{U}=0$ for $k>\operatorname{dim} S\left(\right.$ resp. $\left.H^{k} i_{S}^{!} \mathscr{M}\right|_{U}=0$ for $k<\operatorname{dim} S$ ).

Here $S$ may be assumed irreducible by definition. For the proof we use [3, 1.4.10] and a stratification associated to each $\mathscr{M} \in D^{b} \operatorname{MHM}(X)$. We can also check that (4.6.2) is equivalent to:

$$
H^{k} i_{x}^{*} \mathscr{M}=0 \text { for } k>0 \text { (resp. } k<0 \text { ) for any } x \in X \text {, i.e. } \mathscr{H}^{k} \operatorname{rat}(\mathscr{M})=0
$$


for $k>0$ (resp. $k<0$ ).

Here we use again the distinguished triangles in (4.4.1) and the stratification as above. Then this $t$-structure corresponds to the classical (i.e. not perverse) $t$ structure on $D_{c}^{b}\left(\mathbb{Q}_{X}\right)$.

4.7. Remark. The condition of Steenbrink-Zucker [22, (3.13)] for good variation of mixed Hodge structure is reasonable only in the case where the local monodromies around the points at infinity are unipotent. In fact, the definition of $\tilde{\Psi}$ is not clear in the quasi-unipotent case, and if we interpret the condition (3.13) ii) so that the filtrations $F$ and $W$ extended to those on Deligne's canonical extension (i.e. the eigenvalues of the residue of the connection are contained in $[0,1)$ ) and $\operatorname{Gr}_{p}^{F} \mathrm{Gr}_{k}^{W}$ are free on $\Delta$, this condition is not stable by the base changes of $\Delta$, and we have an example that the conditions in (3.13) are satisfied, but the decomposition (4.11) is not compatible with the Hodge filtration $F$ :

Example. Let $S$ be a Riemann surface, and $t$ a local coordinate around $0 \in S$. Put $S^{*}=S \backslash\{0\}$. Let $L_{0}$ and $L_{1}$ be local systems on $S^{*}$ underlying variations of Hodge structures of weight 0 and 1 , and rank 1 and 2 respectively. Let $\mathscr{L}_{i}$ be Delinge's canonical extension of $L_{i}$ as above, so that the Hodge filtration $F_{p}$ is extended to a holomorphic subbundle of $\mathscr{L}_{i}(i=0$, 1). Let $T_{i}$ be the local monodromy of $L_{i}$ around 0 . We assume that $T_{0}=\mathrm{id}$, $T_{1}$ is semi-simple, 1 is not an eigenvalue of $T_{1}$, and $\operatorname{Gr}_{F}^{p} \mathscr{L}_{1}=0$ for $p \neq 0$, 1. (For example, $L_{1}$ is associated to a family of elliptic curves constructed by Kodaira.) If there is an $\mathcal{O}_{S}$-linear morphism $\phi: F^{1} \mathscr{L}_{1} \rightarrow \mathscr{L}_{0}$ inducing an isomorphism near 0 (e.g. $S$ is an open disc), we define a Hodge filtration $F$ and a weight filtration $W$ on $\mathscr{L}:=\mathscr{L}_{0} \oplus \mathscr{L}_{1}$ by

$$
\begin{aligned}
& F^{2}=0, F^{1}=\operatorname{Im}\left((\phi+\mathrm{id}): F^{1} \mathscr{L}_{1} \rightarrow \mathscr{L}\right), F^{0}=\mathscr{L}, \\
& W_{-1}=0, W_{0}=\mathscr{L}_{0}, W_{1}=\mathscr{L} .
\end{aligned}
$$

Then the conditions (3.13) in [22] are satisfied (if we interpret (3.13) ii) as above). But they are not satisfied for the pull-back of $\mathscr{L}_{S_{S^{*}}}$ to a finite covering of $S^{*}$ so that the local monodromy becomes unipotent. Moreover we can check that the decomposition (4.11) in [loc. cit] is not compatible with the Hodge filtration $F$ in this case. Note that in [loc. cit] they have not proved the compatibility of the decomposition (4.11) with the Hodg!e filtration $F$, which seems to be essential for the proof of the theorem (4.1). In fact we have to prove the compatibility of the three filtration $F, W, V$ on Deligne's extension (where $V$ is the $m$-adic filtration in the unipotent monodromy case), because they use implicitly the isomorphism $F^{p} \mathrm{Gr}_{k}^{W} \mathrm{Gr}_{\mathrm{v}}^{0}=F^{p} \mathrm{Gr}_{V}^{0} \mathrm{Gr}_{k}^{W}$ which is not true if they are not compatible, cf. [9]. Here $W$ is the modified filtration which gives the weight filtration on $\mathscr{L} \otimes \Omega_{S}^{1}(\log D)$, cf. [loc. cit](see also 2.8-9), and $\mathrm{Gr}_{V}^{0}$ corresponds to taking the residue at 0 in the unipotent monodromy case. 


\section{Appendix}

By

\section{Masaki KASHIWARA}

In this appendix we give a proof of Proposition 3.19 which is used essentially in the proof of Theorem 3.20.

A.1. In this appendix, $\tilde{H}_{I}, \tilde{S}_{I}$ will be denoted by $\tilde{H}, \tilde{S}$ for simplicity, and $N$ is replaced by $s$. We may assume $m_{i}=1(i \in I)$ by replacing $N_{i}$ with $m_{i}^{-1} N_{i}$. We choose $i=\sqrt{-1}$ so that the Tate twists $(n)=\otimes(2 \pi i)^{n} \mathbb{Z}$ are trivialized. In particular, $N_{i}$, $s$, etc. are defined over $\mathbb{R}$. By definition we have

$$
\tilde{H}=H[s] / \operatorname{Coker}\left(\prod_{i \in I}\left(s-N_{i}\right)\right) \simeq \bigoplus_{0 \leq j<l} H \otimes s^{j}
$$

(A.1.2) $\tilde{S}\left(s^{j} u, s^{k} v\right)=S\left(u,(-1)^{j} \operatorname{Res}_{s=0}\left(\prod_{i \in I}\left(s-N_{i}\right)^{-1}\right) s^{j+k} v\right)$ for $u, v \in H$,

where $H$ is identified with $H \otimes 1(\subset H[s])$ and $\operatorname{Res}_{s=0}$ is defined by $\operatorname{Res}_{s=0}\left(\sum_{j \in \mathbf{Z}} s^{j} u_{j}\right)=u_{-1}$ for $u_{j} \in H$. Here $\tilde{H}$ has a mixed Hodge structure such that the last isomorphism of (A.1.1) is compatible with it and $s: \widetilde{H} \rightarrow \widetilde{H}(-1)$ is a morphism of mixed Hodge structures. Then we have

(A.1.3) $\quad \tilde{S}\left(u, s^{j} v\right)=S(u, v)(j=l-1)$ and $0(0 \leq j<l-1)$ for $u, v \in H$.

Let $N_{0} \in \sum_{i=1}^{n} \mathbb{R} N_{i}$, and put $N_{i}^{\prime}=N_{i}+N_{0}$. We define a mixed Hodge structure

$$
\tilde{H}^{\prime}=H\left[s^{\prime}\right] / \operatorname{Coker}\left(\prod_{i \in I}\left(s^{\prime}-N_{i}^{\prime}\right)\right) \simeq \bigoplus_{0 \leq j<l} H \otimes s^{\prime j}
$$

and $\widetilde{S}^{\prime}$ as above with $s, N_{i}$ replaced by $s^{\prime}, N_{i}^{\prime}$. Then

A.2. Lemma. We have a natural isomorphism of mixed Hodge structures $\phi: \tilde{H}^{\prime}$ $\rightarrow \widetilde{H}$ such that

$$
\begin{gathered}
\phi\left(\sum_{j \in \mathbf{N}} s^{j} u_{j}\right)=\sum_{j \in \mathbf{N}}\left(s+N_{0}\right)^{j} u_{j} \\
\tilde{S}^{\prime}(\tilde{u}, \tilde{v})=\tilde{S}(\phi(\tilde{u}), \phi(\tilde{v})) \text { for } \tilde{u}, \tilde{v} \in \tilde{H}^{\prime} .
\end{gathered}
$$

Proof. We define $\phi$ by (A.2.1). Then the well-definedness is clear, and (A.2.2) follows from

$$
\operatorname{Res}_{s=0} \phi(\tilde{w})=\operatorname{Res}_{s^{\prime}=0} \tilde{w} \text { for } \tilde{w} \in H\left[s^{\prime}, s^{\prime-1}\right],
$$

where $\phi$ is naturally extended to $H\left[s^{\prime}, s^{-1}\right] \rightarrow H\left[s, s^{-1}\right]$ using $\left(s+N_{0}\right)^{-1}$ $=s^{-1}\left(\sum_{j \geq 0}\left(-N_{0}\right)^{j} s^{-j}\right)$.

A.3. Proof of Proposition 3.19. We apply Lemma A.2 to any $N_{0}=\sum a_{i} N_{i}$ with 
$a_{i}>0$. Then by [6], the assertion is reduced to the following assertions

$$
s^{k}: \mathrm{Gr}_{w+1-l+k}^{W} \tilde{H} \stackrel{\sim}{\longrightarrow} \mathrm{Gr}_{w+1-l-k}^{W} \tilde{H} \text { for any } k \geq 0
$$

(A.3.2) $\quad \tilde{S}\left(\mathrm{id} \otimes s^{k}\right)$ is a polarization on the primitive part $P \mathrm{Gr}_{w+1-l+k}^{W} \tilde{H}:=$ $\operatorname{Kcr} s^{k+1}$ for any $k \geq 0$,

under the assumption

$$
\begin{aligned}
& N: \mathrm{Gr}_{w+k}^{W} H \stackrel{\sim}{\longrightarrow} \mathrm{Gr}_{w-k}^{W} H(k \geq 0) \text { and } S\left(\mathrm{id} \otimes N^{k}\right) \text { is a polarization on } \\
& P \mathrm{Gr}_{w+k}^{W} H(k \geq 0) \text { for any } N=\sum_{i \in I} a_{i} N_{i} \text { with } a_{i} \geq 0 .
\end{aligned}
$$

A.4. Proof of (A.3.1). We proceed by induction on $l$ and $\operatorname{dim} H$. The assertion is clear if $\operatorname{dim} H=0$, and we may assume $\operatorname{dim} H>0 . \quad$ If $l=1$, the natural isomorphism $H \stackrel{\sim}{\longrightarrow} \tilde{H}$ is compatible with $S, \widetilde{S}$, and the assertion is clear. Assume $l>1$. Take $i \in I$ and put $I^{\prime}=I \backslash\{i\}$. We define $\tilde{H}^{\prime \prime}$ $=H[s] / \operatorname{Coker}\left(\prod_{i \in I^{\prime}}\left(s-N_{i}\right)\right)$ and $\widetilde{S}^{\prime \prime}$ as above with $I$ replaced by $I^{\prime}$. Then we have a natural morphism of mixed Hodge structures $\psi: \widetilde{H} \rightarrow \widetilde{H}^{\prime \prime}$ induced by the identity on $H[s]$. On the other hand, by $[8,1.16][15,2.1 .5]$, we have uniquely $\left(H^{\prime} ; N_{1}, \ldots, N_{n} ; S^{\prime}\right)$ a nilpotent orbit of weight $w+1$ with surjective (resp. injective) morphism of mixed Hodge structures can: $H \rightarrow H^{\prime}$ (resp. Var: $H^{\prime} \rightarrow H$ (-1)) compatible with $N_{j}$ such that $\operatorname{Var} \circ \operatorname{can}=N_{i}$, can $\circ \operatorname{Var}=N_{i}$ and $S^{\prime}($ can $\otimes \mathrm{id})=S(\mathrm{id} \otimes \operatorname{Var})$. We define $\tilde{H}^{\prime}, \tilde{S}^{\prime}$ as above with $H$ replaced by $H^{\prime}$. Then the assertion holds for $\tilde{H}^{\prime}, \tilde{S}^{\prime}$ by inductive hypothesis, and can, Var are naturally extended to can $: \widetilde{H} \rightarrow \widetilde{H}^{\prime}$, Var $: \widetilde{H}^{\prime} \rightarrow \widetilde{H}(-1)$ by can $\otimes$ id, etc. so that $\tilde{\operatorname{Var}} \circ \tilde{c a n}=N_{i}$, $\tilde{\text { can }} \circ \widetilde{\operatorname{Var}}=N_{i}$ and $\tilde{S^{\prime}}(\tilde{c a n} \otimes$ id $)=\tilde{S}($ id $\otimes \tilde{\operatorname{Var}})$.

Now take $\tilde{u}=\sum_{0 \leq j<l} s^{j} u_{j} \in \operatorname{Ker} s^{k} \subset \mathrm{Gr}_{w+1-l+k}^{W} \tilde{H}$ with $u_{j} \in \mathrm{Gr}_{w+1-l+k+2 j}^{W}$ $H$, and put $\tilde{v}=s^{k-1} \tilde{u}$. Then $s \tilde{v}=0$ implies

$$
\begin{aligned}
& \tilde{v}=s^{-1}\left(\prod_{j \in I}\left(s-N_{j}\right)-\prod_{j \in I}\left(-N_{j}\right)\right) v \text { for } v \in \mathrm{Gr}_{w+1+l-k}^{W} H \text { such that } \\
& \left(\prod_{j \in I} N_{j}\right) v=0 .
\end{aligned}
$$

By inductive hypothesis we get

$$
\begin{aligned}
& 0 \leq \tilde{S}^{\prime}(\tilde{\operatorname{can}}(\tilde{u}), C \operatorname{can}(\tilde{v}))=\tilde{S}\left(\tilde{u}, C N_{i} \tilde{v}\right)=\tilde{S}\left(u_{0}, C N_{i} \tilde{v}\right)=S\left(u_{0}, C N_{i} v\right) \\
& 0 \leq \tilde{S}^{\prime \prime}(\psi(\tilde{u}), C \psi(\tilde{v}))=\tilde{S}^{\prime \prime}\left(u_{0}, C \psi(\tilde{v})\right)=-S\left(u_{0}, C N_{i} v\right)
\end{aligned}
$$

because $\quad \tilde{v}=\prod_{j \in I^{\prime}}\left(s-N_{j}\right) v-s^{-1}\left(\prod_{j \in I^{\prime}}\left(s-N_{j}\right)-\prod_{j \in I^{\prime}}\left(-N_{j}\right)\right) N_{i} v$. Therefore we get $\tilde{\operatorname{can}}(\tilde{u})=0, \psi(\tilde{u})=0$, which imply $u_{j} \in \operatorname{Ker} \operatorname{Gr}^{W} N_{i} \subset \mathrm{Gr}^{W} H$ and $\tilde{u}$ $=\prod_{j \in I^{\prime}}\left(s-N_{j}\right) u$ for $u \in \mathrm{Gr}_{w+l-1+k}^{W} H$. Thus we get $u=u_{l-1}=0$ by $l-1+k$ $>0$, and hence $\tilde{u}=0$. Then (A.3.1) follows from $\operatorname{dim} \mathrm{Gr}_{w+1-l+k}^{W} \tilde{H}$ $=\operatorname{dim} \mathrm{Gr}_{w+1-l-k}^{W} \tilde{H}$. 
A.5. Proof of (A.3.2). By (A.3.1) and the continuity of eigenvalues, it is enough to prove (A.3.2) under the assumption $N_{i}=N_{j}$ for $i, j \in I$. Put $N=N_{i}, N^{\prime}=s$ $-N$. Let $\left(\mathbb{R}\left[N^{\prime}\right] /\left(N^{\prime l}\right) ; N^{\prime} ; S^{\prime}\right)$ denote a nilpotent orbit of weight $1-l$ such that $S^{\prime}\left(N^{\prime}, N^{\prime j}\right)=(-1)^{i}$ (if $i+j=l-1$ ) and 0 (otherwise). Then we have

$$
(\tilde{H}, \tilde{S})=(H, S) \otimes\left(\mathbb{R}\left[N^{\prime}\right] /\left(N^{\prime \prime}\right), S^{\prime}\right)
$$

and the assertion follows from the commutativity of tensor with limiting mixed Hodge structures.

\section{References}

[1] Beilinson, A.A., On the derived category of perverse sheaves (preprint).

[2] - Notes on absolute Hodge cohomology, Contemporary Mathematics, 55 (1986), 35-68.

[3] Beilinson, A.A., Bernstein, J. and Deligne, P., Faisceaux pervers, Astérisque, 100 (1982), 5171.

[4] Bernstein, J., Algebraic theory of $\mathscr{D}$-Modules (preprint).

[5] Borel, A., Algebraic on $\mathscr{D}$-Modules, Academic Press, Boston, 1987.

[6] Cattani, E. and Kaplan, A., Polarized Mixed Hodge structures and the local monodromy of a variation of Hodge structure, Invent. Math., 67 (1982), 101-115.

[7] Cattani, E., Kaplan, A. and Schmid, W., Degeneration of Hodge structure, Ann. of Math., 123 (1986), 457-535.

[8] - $L^{2}$ and intersection cohomologies for a polarizable variation of Hodge structure, Invent. Math., 87 (1987), 217-252.

[9] Deligne, P., Théorie de Hodge I, Actes Congrès Intern. Math., (1970), 425-430; II, Publ. Math. IHES, 40 (1971), 5-58; III, ibid., 44 (1974), 5-77.

[10] - La conjecture de Weil II, Publ. Math. IHES, 52 (1980), 137-252.

[11] - Equations différentielles à points singuliers réguliers, Lect. Notes in Math., 163, Springer, Berlin, 1970.

[12] Kashiwara, M., Vanishing cycle sheaves and holonomic systems of differential equations, in Lect. Notes in Math., 1016, 134-142, Springer, Berlin, 1983.

[13] - A study of variation of mixed Hodge structure, Publ. RIMS, 22 (1986), 991-1024.

[14] Kashiwara, M. and Kawai, T., On the holonomic system of microdifferential equations III, Publ. RIMS, 17 (1981), 813-979.

[15] - The Poincaré lemma for a variation of Hodge structure, Publ. RIMS, 23 (1987), $345-407$.

[16] - Hodge structure and holonomic systems, Proc. Japan Acad., 62 Ser. A (1985), 14.

[17] MacPherson, R. and Vilonen, K., Elementary construction of perverse sheaves, Invent. Math., 84 (1986), 403-435.

[18] Mebkhout, Z., Une autre équivalence de catégories, Comp. Math., 51 (1984), 63-88.

[19] Saito, M., Modules de Hodge polarisables, Publ. RIMS, 24 (1988), 849-995.

[20] - Mixed Hodge Modules, Proc. Japan Acad., 62 Ser. A (1986), 360-363.

[21] - On the derived categories of Mixed Hodge Modules, Proc. Japan Acad., 62 Ser. A (1986), 364-366.

[22] Steenbrink, J and Zucker, S., Variation of mixed Hodge structure I, Invent. Math., 80 (1985), 485-542. 
[23] Verdier, J.-L., Extension of a perverse sheaf over a closed subspace, Astérisque, 130 (1985), 210-217.

[24] Deligne, P., Théorème de Lefschetz et critères de dégénérescence de suites spectrales., Publ. Math. IHES, 35 (1968), 107-126.

[25] Saito, M., Duality for vanishing cycle functors, Publ. RIMS., 25 (1989), 889-921

[26] — Decomposition theorem for proper Kähler morphisms (IHES preprint July 1988). 
